\title{
Scope and Facial Selectivity of the Prins- Pinacol Synthesis of Attached Rings
}

\author{
Larry E. Overman ${ }^{*}$ and Emile J. Velthuisen \\ Department of Chemistry, 516 Rowland Hall \\ University of California, Irvine, California 92697-2025. \\ Supporting Information \\ (122 Pages)
}

\begin{abstract}
A. Experimental procedures and tabulated characterization data for new compounds not reported in the Experimental Section: p S2-S13

B. Copies of ${ }^{1} \mathrm{H}$ and ${ }^{13} \mathrm{C}$ NMR spectra for all new compounds: $\mathrm{p}$ S15-S122
\end{abstract}




\section{A. Experimental Procedures and Tabulated Characterization Data for New Compounds not Reported in the Experimental Section ${ }^{1}$}

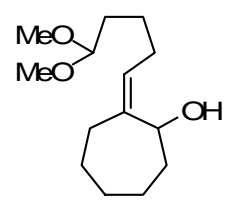

$\mathrm{C}_{14} \mathrm{H}_{26} \mathrm{O}_{3}$ Mol. Wt: 242354

Z-[2-(5,5-Dimethoxypentylidene)cycloheptanol] (18). Following the general procedure, ketone $\mathbf{9}^{2}(1.0 \mathrm{~g}, 7.1 \mathrm{mmol})$ was transformed to $1.0 \mathrm{~g}$ $(59 \%)$ of enone 15. Diagnostic characterization data: ${ }^{1} \mathrm{H}$ NMR $(500 \mathrm{MHz}$, $\left.\mathrm{CDCl}_{3}\right) \delta 5.68(\mathrm{t}, J=7.4 \mathrm{~Hz}, 1 \mathrm{H}), 4.30(\mathrm{t}, J=5.8 \mathrm{~Hz}, 1 \mathrm{H}), 3.24(\mathrm{~s}, 6 \mathrm{H})$; FT-IR (neat) $1685 \mathrm{~cm}^{-1}$. Subsequent reduction yielded $0.60 \mathrm{~g}(60 \%)$ of alcohol 18 as a colorless oil: ${ }^{1} \mathrm{H}$ NMR $\left(500 \mathrm{MHz}, \mathrm{CDCl}_{3}\right) \delta 5.31(\mathrm{t}, J=7.6$ $\mathrm{Hz}, 1 \mathrm{H}), 4.61(\mathrm{~m}, 1 \mathrm{H}), 4.35(\mathrm{t}, J=5.7 \mathrm{~Hz}, 1 \mathrm{H}), 3.30(\mathrm{~s}, 6 \mathrm{H}), 2.25-2.21$ (m, $1 \mathrm{H}), 2.16-2.11(\mathrm{~m}, 2 \mathrm{H}), 2.09-2.05(\mathrm{~m}, 1 \mathrm{H}), 2.0-1.93(\mathrm{~m}, 1 \mathrm{H}), 1.85-1.76(\mathrm{~m}, 1 \mathrm{H})$, 1.69-1.57 (m, $6 \mathrm{H}), 1.44-1.40(\mathrm{~m}, 3 \mathrm{H}), 1.29-1.20(\mathrm{~m}, 1 \mathrm{H}), 1.18-1.08(\mathrm{~m}, 1 \mathrm{H}) ;{ }^{13} \mathrm{C}$ NMR $\left(125 \mathrm{MHz}, \mathrm{CDCl}_{3}\right) \delta 144.1,130.4,104.7,69.1,52.9$, 52.7, 35.2, 33.3, 32.0, 31.4, 30.4, 27.1, 25.1, 23.8; FT-IR (neat) 3424, $1444 \mathrm{~cm}^{-1}$; HRMS (CI) m/z 225.1849 (M-OH, 225.1854 calcd for $\mathrm{C}_{14} \mathrm{H}_{26} \mathrm{O}_{3}$ ). Anal. Calcd for: $\mathrm{C}, 69.38 ; \mathrm{H}, 10.81$; found: $\mathrm{C}, 69.35 ; \mathrm{H}$, 10.64 .

Z-[2-(5,5-Dimethoxypentylidene)cyclooctanol] (19). Following the MeO general procedure, ketone $\mathbf{1 0}^{2}(1.0 \mathrm{~g}, 3.7 \mathrm{mmol})$ was transformed to $1.3 \mathrm{~g}$ (79\%) of enone 16. Diagnostic characterization data: ${ }^{1} \mathrm{H}$ NMR $(500 \mathrm{MHz}$, $\left.\mathrm{CDCl}_{3}\right) \delta 5.59(\mathrm{t}, J=7.5 \mathrm{~Hz}, 1 \mathrm{H}), 4.35(\mathrm{t}, J=5.6 \mathrm{~Hz}, 1 \mathrm{H}), 3.32(\mathrm{~s}, 6 \mathrm{H})$; FT-IR (neat) $1683 \mathrm{~cm}^{-1}$. Subsequent reduction yielded $0.70 \mathrm{~g}(63 \%)$ of alcohol 19 as a colorless oil: ${ }^{1} \mathrm{H}$ NMR $\left(500 \mathrm{MHz}, \mathrm{CDCl}_{3}\right) \delta 5.35(\mathrm{t}, J=$ $7.4 \mathrm{~Hz}, 1 \mathrm{H}), 4.53(\mathrm{dd}, J=10.44,4.2 \mathrm{~Hz}, 1 \mathrm{H}), 4.38(\mathrm{t}, J=5.7 \mathrm{~Hz}, 1 \mathrm{H})$, Mol. Wt.: 256.3810 $3.31(\mathrm{~s}, 6 \mathrm{H}), 2.27-2.08(\mathrm{~m}, 4 \mathrm{H}), 1.97-1.87(\mathrm{~m}, 1 \mathrm{H}), 1.83-1.73(\mathrm{~m}, 2 \mathrm{H}), 1.67-1.34$ (m, $12 \mathrm{H}) ;{ }^{13} \mathrm{C} \mathrm{NMR}\left(125 \mathrm{MHz}, \mathrm{CDCl}_{3}\right) \delta 143.7,130.3,104.9,69.4,53.2,53.0,32.5,31.9$, 31.4, 30.9, 27.4, 25.8, 25.5, 24.9, 24.1; FT-IR (neat) 3420, $1447 \mathrm{~cm}^{-1}$; Anal. Calcd for $\mathrm{C}_{15} \mathrm{H}_{28} \mathrm{O}_{3}$ : C, 70.27; H, 11.01; found: C, 70.33; H, 11.01.

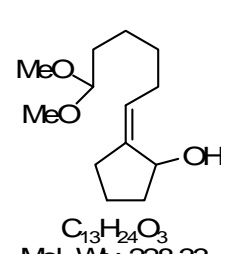

Z-[2-(6,6-Dimethoxyhexylidene)cyclopentanone] (28). Following the general procedure, ketone $24^{3}(1.3 \mathrm{~g}, 11.6 \mathrm{mmol})$ and 5,5-dimethoxypent$1-$ ene $^{4}$ were transformed to $1.0 \mathrm{~g}(39 \%)$ of enone 25. Diagnostic characterization data: ${ }^{1} \mathrm{H}$ NMR $\left(500 \mathrm{MHz}, \mathrm{CDCl}_{3}\right) \delta 5.94(\mathrm{t}, J=7.5 \mathrm{~Hz}, 1$ $\mathrm{H}), 4.35(\mathrm{t}, J=5.7 \mathrm{~Hz}, 1 \mathrm{H}), 3.31(\mathrm{~s}, 6 \mathrm{H})$; FT-IR (neat) $1710 \mathrm{~cm}^{-1}$. Mol. W.: 228.33 Subsequent reduction yielded $0.6 \mathrm{~g}(60 \%)$ of alcohol 28 as a colorless oil: ${ }^{1} \mathrm{H}$ NMR $\left(500 \mathrm{MHz}, \mathrm{CDCl}_{3}\right) \delta 5.41(\mathrm{t}, J=7.6 \mathrm{~Hz}, 1 \mathrm{H}), 4.66(\mathrm{bs}, 1 \mathrm{H}), 4.35(\mathrm{t}, J=5.7$ $\mathrm{Hz}), 3.32$ (s, $6 \mathrm{H}), 2.45-2.41(\mathrm{~m}, 1 \mathrm{H}), 2.19-2.16(\mathrm{~m}, 3 \mathrm{H}), 1.80-1.75$ (m, $3 \mathrm{H}), 1.65-$ $1.59(\mathrm{~m}, 3 \mathrm{H}), 1.43-1.35(\mathrm{~m}, 5 \mathrm{H}) ;{ }^{13} \mathrm{C} \mathrm{NMR}\left(125 \mathrm{MHz}, \mathrm{CDCl}_{3}\right) \delta 145.5,126.2,104.7$, 71.3, 52.9, 52.8, 36.6, 32.5, 31.8, 29.9, 29.4, 24.4, 23.2; FT-IR (neat) 3451, $1463 \mathrm{~cm}^{-1}$; Anal. Calcd for $\mathrm{C}_{13} \mathrm{H}_{24} \mathrm{O}_{3}$ : C, 68.38; H, 10.59; found: C, 68.33; H, 10.51 .

\footnotetext{
${ }^{1}$ General experimental details have been described: MacMillan, D. W. C.; Overman, L. E.; Pennington, L. D. J. Am. Chem. Soc. 2001, 123, 9033-9044.

${ }^{2}$ McKervey, M. A.; Ratananukul, P. Tetrahedron Lett. 1983, 24, 117-120.

${ }^{3}$ Donohoe, T. J.; Raoof, A.; Linney, J. D.; Helliwell, M.; Org. Lett. 2001, 6, 861-864.

${ }^{4}$ Price, C.C.; Balsley, R. B. J. Org. Chem. 1966, 31, 3406-3407.
} 
Z-[2-(6,6-dimethoxyhexylidene)cycloheptanone] (29). Following the general procedure, ketone $9(1.0 \mathrm{~g}, 7.1 \mathrm{mmol})$ and 5,5-dimethoxypent-1-ene were transformed to

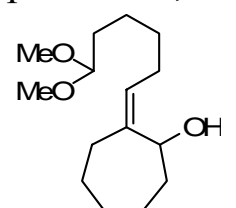

$\mathrm{C}_{15} \mathrm{H}_{28} \mathrm{O}_{3}$ Mol. W.: 256.38

$1.2 \mathrm{~g}(66 \%)$ of enone 26. Diagnostic characterization data: ${ }^{1} \mathrm{H}$ NMR (500 $\left.\mathrm{MHz}, \mathrm{CDCl}_{3}\right) \delta 5.75(\mathrm{t}, J=7.3 \mathrm{~Hz}, 1 \mathrm{H}), 4.35(\mathrm{t}, J=5.7 \mathrm{~Hz}, 1 \mathrm{H}), 3.31(\mathrm{~s}$, $6 \mathrm{H})$; FT-IR (neat) $1683 \mathrm{~cm}^{-1}$. Subsequent reduction yielded $1.0 \mathrm{~g}(80 \%)$ of alcohol 29 as a colorless oil: ${ }^{1} \mathrm{H}$ NMR $\left(500 \mathrm{MHz} \mathrm{CDCl}_{3}\right) \delta 5.35(\mathrm{t}, J=7.4$ $\mathrm{Hz}, 1 \mathrm{H}), 4.64-4.61(\mathrm{~m}, 1 \mathrm{H}), 4.35(\mathrm{t}, J=5.7 \mathrm{~Hz}, 1 \mathrm{H}), 3.31(\mathrm{~s}, 6 \mathrm{H})$, $5 \mathrm{H}), 1.43-1.35(\mathrm{~m}, 6 \mathrm{H}), 1.28-1.25(\mathrm{~m}, 1 \mathrm{H}), 1.16-1.13(\mathrm{~m}, 1 \mathrm{H}) ;{ }^{13} \mathrm{C}$ NMR $(125 \mathrm{MHz}$, $\left.\mathrm{CDCl}_{3}\right) \delta 143.9,130.8,104.7,69.2,52.9,52.8,35.2,33.3,32.6,31.5,30.5,29.9,27.4$, 24.4, 23.8; FT-IR (neat) 3432, $1447 \mathrm{~cm}^{-1}$; HRMS (CI) m/z 256.2395 (M, 256.2402 calcd for $\mathrm{C}_{16} \mathrm{H}_{28} \mathrm{O}_{3}$ ). Anal. Calcd for $\mathrm{C}_{15} \mathrm{H}_{28} \mathrm{O}_{3}$ : C, 70.27; $\mathrm{H}, 11.09$; found: $\mathrm{C}, 71.21 ; \mathrm{H}, 11.09$.

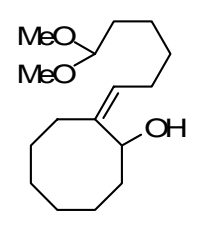

$\mathrm{C}_{16} \mathrm{H}_{30} \mathrm{O}_{3}$ Mol. Wh: 270.4076

Z-[2-(6,6-Dimethoxyhexylidene)cyclooctanol] (30). Following the general procedure, ketone 10 (1.0 g, $6.5 \mathrm{mmol})$ and 5,5-dimethoxypent-1ene were transformed to $0.8 \mathrm{~g}(46 \%)$ of enone 27. Diagnostic characterization data: ${ }^{1} \mathrm{H} \mathrm{NMR}\left(500 \mathrm{MHz}, \mathrm{CDCl}_{3}\right) \delta 5.58(\mathrm{t}, J=7.4 \mathrm{~Hz}, 1$ $\mathrm{H}), 4.34(\mathrm{t}, J=5.7 \mathrm{~Hz}, 1 \mathrm{H}), 3.30(\mathrm{~s}, 6 \mathrm{H})$; FT-IR (neat) $1683 \mathrm{~cm}^{-1}$. ${ }^{1} \mathrm{H}$ NMR (500 $\left.\mathrm{MHz}, \mathrm{CDCl}_{3}\right) \delta 5.35(\mathrm{t} J=7.4 \mathrm{~Hz})$, $4.35(\mathrm{t}, J=5.7 \mathrm{~Hz}, 1 \mathrm{H}), 3.31(\mathrm{~s}, 6 \mathrm{H}), 2.28-2.21(\mathrm{~m}, 1 \mathrm{H}), 2.16-2.14(\mathrm{~m}, 3 \mathrm{H}), 1.97-$ $1.87(\mathrm{~m}, 1 \mathrm{H}), 1.83-1.75(\mathrm{~m}, 2 \mathrm{H}), 1.53-1.35(\mathrm{~m}, 14 \mathrm{H}) ;{ }^{13} \mathrm{C} \mathrm{NMR}\left(125 \mathrm{MHz}, \mathrm{CDCl}_{3}\right) \delta$ 143.1, 130.3, 104.7, 69.1, 53.0, 52.8, 32.5, 31.7, 31.1, 30.7, 29.9, 27.3, 25.54, 25.52, 24.4, 23.9; FT-IR (neat) 3416, $1447 \mathrm{~cm}^{-1}$; HRMS (CI) m/z $252.2092\left(\mathrm{M}-\mathrm{H}_{2} \mathrm{O}, 252.2089\right.$ calcd for $\mathrm{C}_{16} \mathrm{H}_{30} \mathrm{O}_{3}$ ).

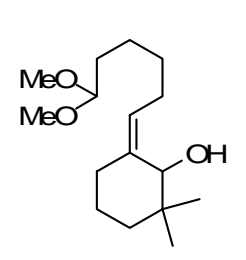

$\mathrm{C}_{16} \mathrm{H}_{30} \mathrm{O}_{3}$

Z-[6-(6,6-Dimethoxyhexylidene)-2,2-dimethylcyclohexanol]

(44). Following the general procedure, ketone $35^{5}(1.8 \mathrm{~g}, 12 \mathrm{mmol})$ and 5,5dimethoxypent-1-ene were transformed to $1.0 \mathrm{~g}(32 \%)$ of enone 41. Diagnostic characterization data: ${ }^{1} \mathrm{H}$ NMR $\left(500 \mathrm{MHz}, \mathrm{CDCl}_{3}\right) \delta 7.51(\mathrm{t}, J$ $=7.5 \mathrm{~Hz}, 1 \mathrm{H}), 4.32(\mathrm{t}, J=5.7 \mathrm{~Hz}, 1 \mathrm{H}), 3.29(\mathrm{~s}, 6 \mathrm{H})$; FT-IR (neat) 1683 $\mathrm{cm}^{-1}$. Subsequent reduction yielded $0.5 \mathrm{~g}(95 \%)$ of alcohol 62 as a colorless oil: ${ }^{1} \mathrm{H}$ NMR $\left(500 \mathrm{MHz}, \mathrm{CDCl}_{3}\right) \delta 5.28(\mathrm{td}, J=7.6,1.9 \mathrm{~Hz}, 1 \mathrm{H})$, $4.36(\mathrm{t}, J=5.7 \mathrm{~Hz}, 1 \mathrm{H}), 4.07(\mathrm{~s}, 1 \mathrm{H}), 3.32(\mathrm{~s}, 6 \mathrm{H}), 2.43-2.31(\mathrm{~m}, 1 \mathrm{H}), 2.18-2.0(\mathrm{~m}, 2$ $\mathrm{H}), 1.95-1.90(\mathrm{~m}, 1 \mathrm{H}), 1.78-1.70(\mathrm{~m}, 2 \mathrm{H}), 1.65-1.58(\mathrm{~m}, 2 \mathrm{H}), 1.48-1.36(\mathrm{~m}, 6 \mathrm{H})$, $1.18-1.10(\mathrm{~m}, 1 \mathrm{H}), 1.01(\mathrm{~s}, 3 \mathrm{H}), 0.81(\mathrm{~s}, 3 \mathrm{H}) ;{ }^{13} \mathrm{C} \mathrm{NMR}\left(125 \mathrm{MHz}, \mathrm{CDCl}_{3}\right) \delta 138.0$, 126.4, 104.8, 73.9, 72.4, 53.1, 52.8, 35.9, ; FT-IR (neat) 3466, $1451 \mathrm{~cm}^{-1}$; HRMS (CI) $\mathrm{m} / \mathrm{z} 252.2090\left(\mathrm{M}-\mathrm{H}_{2} \mathrm{O}, 252.2089\right.$ calcd for $\left.\mathrm{C}_{16} \mathrm{H}_{30} \mathrm{O}_{3}\right)$. Anal. Calcd for: $\mathrm{C}, 71.07 ; \mathrm{H}$, 11.18; found: C, 71.30; H, 11.34.

\footnotetext{
${ }^{5}$ Johnson, W. S.; Posvic, H. J. Am. Chem. Soc. 1947, 69, 1361-1366.
} 

Following the general procedure, ketone $36^{6}(2.0 \mathrm{~g}, 13 \mathrm{mmol})$ and $5,5-$ dimethoxypent-1-ene were transformed to $1.93 \mathrm{~g}(55 \%)$ of enone 42. Diagnostic characterization data: ${ }^{1} \mathrm{H}$ NMR $\left(500 \mathrm{MHz}, \mathrm{CDCl}_{3}\right) \delta 5.58(\mathrm{ddd}$, $J=9.0,7.6,1.5 \mathrm{~Hz}, 1 \mathrm{H}), 4.31\left(\mathrm{t}, J=5.9 \mathrm{~Hz}, 1 \mathrm{H}\right.$ ); FT-IR (neat) $1691 \mathrm{~cm}^{-}$ 1. A portion of enone $42(0.7 \mathrm{~g}, 2.7 \mathrm{mmol})$ was reduced to provide $0.7 \mathrm{~g}$ mol. W.: 270.4076 (96\%) of alcohol 45 as a colorless oil: ${ }^{1} \mathrm{H}$ NMR $\left(500 \mathrm{MHz}, \mathrm{CDCl}_{3}\right) \delta 5.20$ (t, $J=7.6 \mathrm{~Hz}, 1 \mathrm{H}), 4.68(\mathrm{bs}, 1 \mathrm{H}), 4.34(\mathrm{t}, J=5.7 \mathrm{~Hz}, 1 \mathrm{H}), 3.30$ (s, $6 \mathrm{H}), 2.58-2.50$ (m, $1 \mathrm{H}), 2.12-2.06(\mathrm{~m}, 2 \mathrm{H}), 1.95-1.83(\mathrm{~m}, 1 \mathrm{H}), 1.73-1.63(\mathrm{~m}, 1 \mathrm{H}), 1.60-1.54(\mathrm{~m}, 2 \mathrm{H})$, 1.47-1.45 (m, $2 \mathrm{H}), 1.37-1.32$ (m, $5 \mathrm{H}), 1.24-1.23$ (m, $1 \mathrm{H}), 1.16$ (s, $3 \mathrm{H}), 0.88$ (s, $3 \mathrm{H})$; ${ }^{13} \mathrm{C}$ NMR $\left(125 \mathrm{MHz}, \mathrm{CDCl}_{3}\right) \delta 140.0,125.5,104.7,66.5,53.0,52.7,46.2,41.1,33.1$, 32.4, 30.8, 30.6, 29.3, 27.6, 26.8, 24.1; FT-IR (neat) $3451,1455 \mathrm{~cm}^{-1}$; HRMS (CI) $\mathrm{m} / \mathrm{z}$ 252.2099 $\left(\mathrm{M}-\mathrm{H}_{2} \mathrm{O}, 252.2089\right.$ calcd for $\mathrm{C}_{16} \mathrm{H}_{30} \mathrm{O}_{3}$. Anal. Calcd for $\mathrm{C}_{16} \mathrm{H}_{30} \mathrm{O}_{3}: \mathrm{C}, 71.07 ; \mathrm{H}$, 11.18; found: C, 71.91; H, 11.38.

\section{Z-[2-(6,6-Dimethoxyhexylidene)-4,4-dimethylcyclohexanol]}

(46).

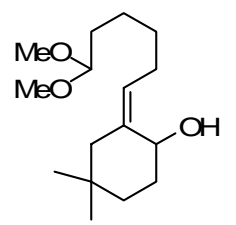

Following the general procedure described above, ketone $37^{7}(2.0 \mathrm{~g}, 13$ $\mathrm{mmol})$ and 5,5-dimethoxypent-1-ene were transformed to $0.74 \mathrm{~g} \mathrm{(88 \% )} \mathrm{of}$ enone 43. Diagnostic characterization data: ${ }^{1} \mathrm{H}$ NMR $\left(500 \mathrm{MHz}, \mathrm{CDCl}_{3}\right) \delta$ $5.54(\mathrm{t}, J=7.4 \mathrm{~Hz}, 1 \mathrm{H}), 4.34(\mathrm{t}, J=5.7 \mathrm{~Hz}, 1 \mathrm{H}), 3.30(\mathrm{~s}, 6 \mathrm{H})$; FT-IR (neat) $1702 \mathrm{~cm}^{-1}$. A portion of enone $43(0.7 \mathrm{~g}, 2.7 \mathrm{mmol})$ was reduced to $\mathrm{C}_{16} \mathrm{H}_{30} \mathrm{O}_{3}$ provide $0.72 \mathrm{~g}(96 \%)$ of alcohol $\mathbf{4 6}$ as a colorless oil: ${ }^{1} \mathrm{H}$ NMR $(500 \mathrm{MHz}$, Mol. Wt: 270.4076 $\left.\mathrm{CDCl}_{3}\right) \delta 5.14(\mathrm{td}, J=7.5,1.7 \mathrm{~Hz}, 1 \mathrm{H}), 4.64(\mathrm{br} \mathrm{s}, 1 \mathrm{H}), 4.34(\mathrm{t}, J=5.7 \mathrm{~Hz}, 1 \mathrm{H}), 3.31$ (s, $6 \mathrm{H}), 2.37$ (d, $J=13.2 \mathrm{~Hz}, 1 \mathrm{H}), 2.13-2.01(\mathrm{~m}, 2 \mathrm{H}), 1.82-1.65(\mathrm{~m}, 2 \mathrm{H}), 1.65-1.50$ $(\mathrm{m}, 5 \mathrm{H}), 1.42-1.30(\mathrm{~m}, 4 \mathrm{H}), 0.94(\mathrm{~s}, 3 \mathrm{H}), 0.78(\mathrm{~s}, 3 \mathrm{H}),{ }^{13} \mathrm{C} \mathrm{NMR}\left(125 \mathrm{MHz}, \mathrm{CDCl}_{3}\right) \delta$ 138.4, 126.6, 104.7, 64.7, 53.1, 52.7, 45.4, 33.5, 32.48, 32.45, 32.3, 29.92, 29.91, 26.8, 23.8; FT-IR (neat) $3466 \mathrm{~cm}^{-1}$; Anal. Calcd for $\mathrm{C}_{16} \mathrm{H}_{28} \mathrm{O}_{3}: \mathrm{C}, 71.07$; $\mathrm{H}, 11.18$; found: $\mathrm{C}$, $71.27 ; \mathrm{H}, 10.89$.

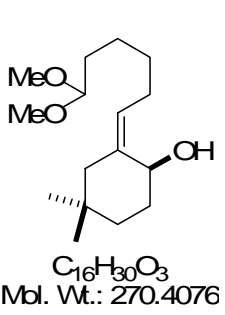

(S)-Z-[2-(6,6-Dimethoxyhexylidene)-4,4-dimethylcyclohexanol] (46). A solution of $(R)$-CBS catalyst $(2.0 \mathrm{~mL}, 2.0 \mathrm{mmol}, 1.0 \mathrm{M} \text { in PhMe })^{8}$ was added dropwise to a solution of $\mathrm{BH}_{3} \cdot \mathrm{THF}(2 \mathrm{~mL}, 2.0 \mathrm{mmol}, 1.0 \mathrm{M}$ in THF) in $\mathrm{CH}_{2} \mathrm{Cl}_{2}(3 \mathrm{~mL})$ at room temperature. The reaction mixture was cooled to $-36{ }^{\circ} \mathrm{C}$ and maintained for $30 \mathrm{~min}$. A solution of ketone $43(0.5$ $\mathrm{g}, 1.89 \mathrm{mmol})$ in $\mathrm{CH}_{2} \mathrm{Cl}_{2}(3 \mathrm{~mL})$ was added to the reaction mixture via a syringe pump over a 30 min period. After stirring for $18 \mathrm{~h}$, the reaction was poured into saturated aqueous $\mathrm{NaHCO}_{3}$ and the layers were seperated. The aqueous layer was extracted with $\mathrm{CH}_{2} \mathrm{Cl}_{2}(2 \times 10 \mathrm{~mL})$ and the combined organic layers were washed with brine $(50 \mathrm{~mL})$, dried $\left(\mathrm{Na}_{2} \mathrm{SO}_{4}\right)$, and concentrated. The residue was purified via flash chromatography on silica gel (10\% ethyl acetate - hexanes) to yield $0.37 \mathrm{~g}$

\footnotetext{
${ }^{6}$ Seifert, S. Helv. Chim. Acta. 1951, 34, 728-735.

${ }^{7}$ Tietze, L. F.; Peterson, S. Eur. J. Org. Chem. 2001, 9, 1619-1624.

${ }^{8}$ For a review of oxazaborolidine mediated reductions with CBS reagents, see: Corey. E. J.; Helal, C. J. Angew. Chem., Int. Ed. 1998, 37, 1986-2012.
} 
$(74 \%)$ of alcohol $(S)-46$ as a yellow oil: $[\alpha]^{23}$ D $-30.9,[\alpha]^{23}{ }_{577}-32.6,[\alpha]^{23}{ }_{546}-36.1$, $[\alpha]^{23}{ }_{435}-65.2,[\alpha]^{23}{ }_{405}-81.2\left(\mathrm{c} 1.08, \mathrm{CHCl}_{3}\right)$; all specta matched the racemate.

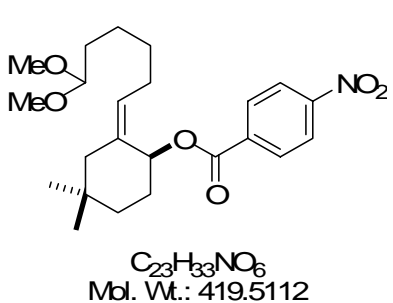

Z-[4-Nitrobenzoic acid 2-(6,6-dimethoxyhexylidene)-4,4dimethylcyclohexyl ester] (71). 4-nitrobenoyl chloride (53 $\mathrm{mg}$, $0.29 \mathrm{mmol})$ was added to a solution of alcohol $46(50 \mathrm{mg}, 0.19$ $\mathrm{mmol})$ and triethylamine $(73 \mu \mathrm{L}, 0.57 \mathrm{mmol})$ in $\mathrm{CH}_{2} \mathrm{Cl}_{2}(2 \mathrm{~mL})$. After stirring for $18 \mathrm{~h}$, the reaction was partitioned between $\mathrm{CH}_{2} \mathrm{Cl}_{2}(5 \mathrm{~mL})$ and $\mathrm{H}_{2} \mathrm{O}(5 \mathrm{~mL})$ and the layers were separated. The combined organic layers were washed with brine $(10 \mathrm{~mL})$, dried $\left(\mathrm{Na}_{2} \mathrm{SO}_{4}\right)$, filtered and concentrated in vacuo. The residue was purified via flash column chromatography on silica gel ( $2 \%$ ethyl acetate - hexanes) to yield $60 \mathrm{mg}(75 \%)$ of the benzoate: ${ }^{1} \mathrm{H}$ NMR $\left(500 \mathrm{MHz}, \mathrm{CDCl}_{3}\right) \delta 8.30(\mathrm{~d}, J=8.8 \mathrm{~Hz}, 2 \mathrm{H}), 8.19(\mathrm{~d}, J=8.6$ $\mathrm{Hz}, 2 \mathrm{H}), 5.96$, (bs, $1 \mathrm{H}), 5.32$ (t, $J=7.3 \mathrm{~Hz}, 1 \mathrm{H}), 4.35$ (t, $J=5.7 \mathrm{~Hz}, 1 \mathrm{H}), 3.30(\mathrm{~s}, 6 \mathrm{H})$, 2.35-2.15 (m, $3 \mathrm{H}), 2.05-2.95(\mathrm{~m}, 1 \mathrm{H}), 2.83-1.71(\mathrm{~m}, 3 \mathrm{H}), 1.64-1.57(\mathrm{~m}, 3 \mathrm{H}), 1.45-$ $1.32(\mathrm{~m}, 4 \mathrm{H}), 1.03(\mathrm{~s}, 3 \mathrm{H}), 0.88(\mathrm{~s}, 3 \mathrm{H}) ;{ }^{13} \mathrm{C} \mathrm{NMR}\left(125 \mathrm{MHz}, \mathrm{CDCl}_{3}\right) \delta 163.8,150.4$, 136.5, 133.3, 130.7, 128.7, 123.6, 104.5, 69.8, 52.7, 46.1, 34.3, 32.4, 32.3, 29.9, 28.0, 27.3, 24.3, 23.9; IR(neat) 1719, 1530, $1273 \mathrm{~cm}^{-1}$; HRMS (ESI) $\mathrm{m} / \mathrm{z} 442.2206(\mathrm{M}+\mathrm{Na}$, 442.2206 calcd for $\left.\mathrm{C}_{23} \mathrm{H}_{33} \mathrm{O}_{6} \mathrm{~N}\right)$.

For the enantioenriched substrate, derived from (S)-46: $[\alpha]^{23}{ }_{D}+10.2,[\alpha]^{23}{ }_{577}+10.6$, $[\alpha]_{546}^{23}+12.3,[\alpha]^{23}{ }_{435}+23.7,[\alpha]^{23}{ }_{405}+29.6\left(\mathrm{c} 0.13, \mathrm{CHCl}_{3}\right)$; all specta matched those of the the racemate.

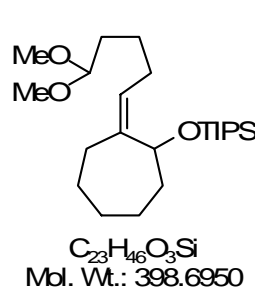

Z-[[2-(5,5-Dimethoxypentylidene)cycloheptyloxy]triisopropylsilane] (21). Following the general procedure, $0.69 \mathrm{~g}(70 \%)$ of silyloxy 21 was isolated as a yellow oil: ${ }^{1} \mathrm{H}$ NMR $\left(500 \mathrm{MHz}, \mathrm{CDCl}_{3}\right) \delta 5.07-5.01(\mathrm{~m}, 1$ $\mathrm{H}), 4.87-4.86(\mathrm{~m}, 1 \mathrm{H}), 4.37(\mathrm{t}, J=5.7 \mathrm{~Hz}, 1 \mathrm{H}), 3.32(\mathrm{~s}, 6 \mathrm{H}), 2.62-$ $2.53(\mathrm{~m}, 1 \mathrm{H}), 2.03-1.89(\mathrm{~m}, 3 \mathrm{H}), 1.89-1.83(\mathrm{~m}, 1 \mathrm{H}), 1.63-1.50(\mathrm{~m}, 6$ $\mathrm{H}), 1.50-1.35(\mathrm{~m}, 4 \mathrm{H}), 1.32-1.22(\mathrm{~m}, 1 \mathrm{H}), 1.07-1.05(\mathrm{~m}, 21 \mathrm{H}) ;{ }^{13} \mathrm{C}$ NMR $\left(125 \mathrm{MHz}, \mathrm{CDCl}_{3}\right) \delta 143.5,125.0,104.7,70.0,52.9,52.7,37.4$, $32.8,32.5,27.9,27.4,27.2,25.2,24.6,18.3,12.5$; IR(neat) 2930, $2868 \mathrm{~cm}^{-1}$; HRMS (ESI) $\mathrm{m} / \mathrm{z} 367.3030\left(\mathrm{M}-\mathrm{OMe}^{+}, 367.3032\right.$ calcd for $\left.\mathrm{C}_{23} \mathrm{H}_{46} \mathrm{O}_{3} \mathrm{Si}\right)$. Anal. Calcd for $\mathrm{C}_{23} \mathrm{H}_{46} \mathrm{O}_{3} \mathrm{Si}: \mathrm{C}, 69.29$; H, 11.63; found: C, 69.42; H, 11.72 .

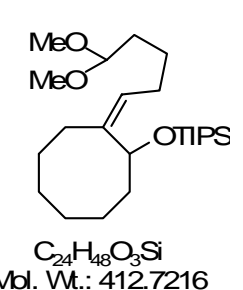

Z-[[2-(5,5-Dimethoxypentylidene)cyclooctyloxy]triisopropylsilane] (22). Following the general procedure, $0.57 \mathrm{~g}(51 \%)$ of silyloxy 22 was isolated as a yellow oil: ${ }^{1} \mathrm{H}$ NMR $\left(500 \mathrm{MHz}, \mathrm{CDCl}_{3}\right) \delta 5.14(\mathrm{t}, J=8.9$ $\mathrm{Hz}, 1 \mathrm{H}), 4.70(\mathrm{dd}, J=7.5,3.7 \mathrm{~Hz}, 1 \mathrm{H}), 4.36(\mathrm{t}, J=5.7 \mathrm{~Hz}, 1 \mathrm{H}), 3.31$ (s, $6 \mathrm{H}), 2.52-2.42(\mathrm{~m}, 1 \mathrm{H}), 2.06(\mathrm{q}, J=14.8,7.4 \mathrm{~Hz}, 2 \mathrm{H}), 2.00-1.91$ $(\mathrm{m}, 1 \mathrm{H}), 1.90-1.74(\mathrm{~m}, 2 \mathrm{H}), 1.73-1.60(\mathrm{~m}, 3 \mathrm{H}), 1.54-1.30(\mathrm{~m}, 8 \mathrm{H})$, $1.08-0.99(\mathrm{~m}, 22 \mathrm{H}) ;{ }^{13} \mathrm{C}$ NMR $\left(125 \mathrm{MHz}, \mathrm{CDCl}_{3}\right) \delta 143.4,126.8,104.9$, 77.7, 70.2, 53.1, 52.9, 36.3, 33.1, 32.8, 28.1, 27.9, 27.6, 26.1, 25.4, 21.5, 18.55, 18.51, 12.8; IR(neat) 2926, $2868 \mathrm{~cm}^{-1}$; HRMS (ESI) m/z $412.3378\left(\mathrm{M}-\mathrm{OMe}^{+}, 412.3372 \mathrm{calcd}\right.$ for $\mathrm{C}_{24} \mathrm{H}_{48} \mathrm{O}_{3} \mathrm{Si}$ ). Anal. Calcd for $\mathrm{C}_{24} \mathrm{H}_{48} \mathrm{O}_{3} \mathrm{Si}$ : C, 69.84; H, 11.72; found: $\mathrm{C}, 70.23 ; \mathrm{H}$, 11.92 . 


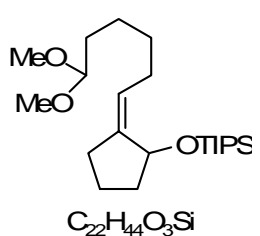

Z-[2-(6,6-Dimethoxyhexylidene)cyclopentyloxy]triisopropylsilane] (31). Following the general procedure, $0.4 \mathrm{~g}(68 \%)$ of silyloxy 31 was isolated as a yellow oil: ${ }^{1} \mathrm{H}$ NMR $\left(500 \mathrm{MHz}, \mathrm{CDCl}_{3}\right) \delta 5.30-5.25(\mathrm{~m}, 1$ $\mathrm{H}), 4.82-4.76(\mathrm{~m}, 1 \mathrm{H}), 4.36(\mathrm{t}, J=7.5 \mathrm{~Hz}, 1 \mathrm{H}), 3.32(\mathrm{~s}, 6 \mathrm{H}), 2.50-2.43$ (m, 1 H), 2.19-2.08 (m, 3 H), 1.87-1.67 (m, 3 H), 1.62-1.53 (m, $2 \mathrm{H})$, Mol. Wt: $384.67 \quad 1.43-1.33(\mathrm{~m}, 4 \mathrm{H}), 1.08-1.06(\mathrm{~m}, 22 \mathrm{H}) ;{ }^{13} \mathrm{C} \mathrm{NMR}\left(125 \mathrm{MHz}, \mathrm{CDCl}_{3}\right) \delta$ 144.7, 124.2, 104.7, 76.9, 72.1, 52.8, 52.7, 37.2, 32.7, 31.3, 30.1, 29.6, 24.6, 22.4, 18.5, 13.0; FT-IR (neat) 2945, $2868 \mathrm{~cm}^{-1}$; HRMS (CI) m/z 384.3057 (M, 384.3059 calcd for $\mathrm{C}_{22} \mathrm{H}_{44} \mathrm{O}_{3} \mathrm{Si}$ ).

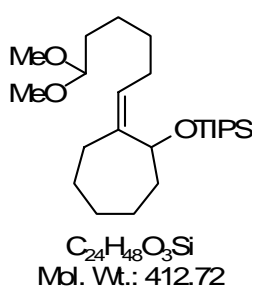

Z-[2-(6,6-dimethoxyhexylidene)cycloheptyloxy]triisopropylsilane] (32). Following the general procedure, $1.25 \mathrm{~g}(80 \%)$ of silyloxy 32 was isolated as a yellow oil: ${ }^{1} \mathrm{H}$ NMR $\left(500 \mathrm{MHz}, \mathrm{CDCl}_{3}\right) \delta 5.04(\mathrm{t}, J=7.1$ $\mathrm{Hz}, 1 \mathrm{H}), 4.89-4.87(\mathrm{~m}, 1 \mathrm{H}), 4.36(\mathrm{t}, J=5.7 \mathrm{~Hz}, 1 \mathrm{H}), 3.22(\mathrm{~s}, 6 \mathrm{H})$, 2.59-2.57 (m, $1 \mathrm{H}), 2.00-1.55(\mathrm{~m}, 8 \mathrm{H}), 1.44-1.29(\mathrm{~m}, 7 \mathrm{H}), 1.07-1.02$ $(\mathrm{m}, 23 \mathrm{H}) ;{ }^{13} \mathrm{C} \mathrm{NMR}\left(125 \mathrm{MHz}, \mathrm{CDCl}_{3}\right) \delta 143.2,125.2,104.7,69.9$, 52.9, 52.8, 37.3, 32.8, 32.7, 30.1, 27.9, 27.6, 27.1, 24.7, 24.6, 18.3, 18.3, 12.5; FT-IR (neat) 2930, $2868 \mathrm{~cm}^{-1}$; Anal. Calcd for $\mathrm{C}_{24} \mathrm{H}_{48} \mathrm{O}_{3} \mathrm{Si}$ : C, 69.84; $\mathrm{H}, 11.72$; found: $\mathrm{C}, 70.10 ; \mathrm{H}, 11.85$.

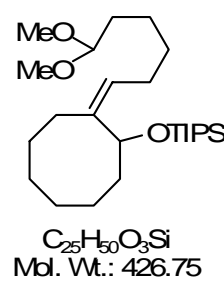

Z-[[2-(6,6-Dimethoxyhexylidene)cyclooctyloxy]triisopropylsilane] (33). Following the general procedure, $0.22 \mathrm{~g}(80 \%)$ of silyloxy 33 was isolated as a yellow oil: ${ }^{1} \mathrm{H} \mathrm{NMR}\left(500 \mathrm{MHz}, \mathrm{CDCl}_{3}\right) \delta 5.14(\mathrm{t}, J=7.2 \mathrm{~Hz}, 1 \mathrm{H})$, 4.73-4.69 (m, $1 \mathrm{H}), 4.36(\mathrm{t}, J=5.7 \mathrm{~Hz}, 1 \mathrm{H}), 3.22(\mathrm{~s}, 6 \mathrm{H}), 2.50-2.44(\mathrm{~m}$, $1 \mathrm{H}), 2.09-1.75(\mathrm{~m}, 5 \mathrm{H}), 1.70-1.32(\mathrm{~m}, 12 \mathrm{H}), 1.06-1.02(\mathrm{~m}, 23 \mathrm{H}) ;{ }^{13} \mathrm{C}$ NMR $\left(125 \mathrm{MHz}, \mathrm{CDCl}_{3}\right) \delta 142.8,126.8,104.8,69.9,52.9,36.0,32.8$, 29.9, 27.9, 27.3, 25.8, 24.8, 21.3, 18.5, 18.3, 12.6; FT-IR (neat) 2926, 2868 $\mathrm{cm}^{-1}$; HRMS (CI) m/z $426.3525\left(\mathrm{M}, 426.3529\right.$ calcd for $\mathrm{C}_{25} \mathrm{H}_{50} \mathrm{O}_{3} \mathrm{Si}$ ). Anal. Calcd for $\mathrm{C}_{25} \mathrm{H}_{50} \mathrm{O}_{3} \mathrm{Si}$ : C, 70.36; H, 11.81; found: C, 70.55; H, 11.90 .

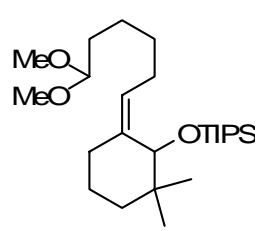

Z-[[6-(6,6-Dimethoxyhexylidene)-2,2-dimethylcyclohexyloxy] triisopropylsilane] (47). Following the general procedure, $0.6 \mathrm{~g}(76 \%)$ of silyloxy 47 was isolated as a yellow oil: ${ }^{1} \mathrm{H} \mathrm{NMR}\left(500 \mathrm{MHz}, \mathrm{CDCl}_{3}\right)$ $\delta 5.16-5.13(\mathrm{~m}, 1 \mathrm{H}), 4.37(\mathrm{t}, J=5.7 \mathrm{~Hz}, 1 \mathrm{H}), 4.17(\mathrm{~s}, 1 \mathrm{H}), 3.32(\mathrm{~s}, 6$ $\mathrm{H}), 2.45-2.40(\mathrm{~m}, 1 \mathrm{H}), 2.13-1.95(\mathrm{~m}, 2 \mathrm{H}), 1.85-1.73(\mathrm{~m}, 2 \mathrm{H}), 1.61-$ $1.36(\mathrm{~m}, 8 \mathrm{H}), 1.09-0.99(\mathrm{~m}, 22 \mathrm{H}), 0.90(\mathrm{~s}, 3 \mathrm{H}), 0.77$ (s, $3 \mathrm{H}) ;{ }^{13} \mathrm{C} \mathrm{NMR}$ $\left(125 \mathrm{MHz}, \mathrm{CDCl}_{3}\right) \delta 139.8,124.5,104.8,75.0,52.9,52.8,37.6,33.8$, Mol. W.:: 426.7482

$32.7,32.0,30.0,28.7,27.7,24.8,24.2,23.9,18.6,18.4,12.9$; FT-IR (neat) 2941, 2868 $\mathrm{cm}^{-1}$; HRMS (EI) $\mathrm{m} / \mathrm{z} 449.3433\left(\mathrm{M}+\mathrm{Na}, 449.3427\right.$ calcd for $\mathrm{C}_{25} \mathrm{H}_{50} \mathrm{O}_{3} \mathrm{SiNa}$ ). Anal. Calcd for $\mathrm{C}_{25} \mathrm{H}_{50} \mathrm{O}_{3} \mathrm{Si}: \mathrm{C}, 70.36$; H, 11.81; found: C, 70.64; H, 11.90 . 
Z-[[6-(6,6-Dimethoxyhexylidene)-3,3-dimethylcyclohexyloxy] triisopropylsilane]

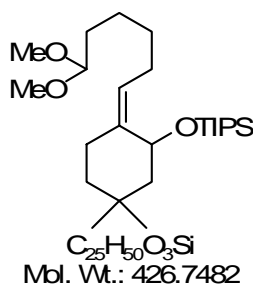

(48). Following the general procedure, $0.63 \mathrm{~g} \mathrm{(91 \% )} \mathrm{of} \mathrm{silyloxy} 48$ was isolated as a yellow oil: ${ }^{1} \mathrm{H}$ NMR $\left(500 \mathrm{MHz}, \mathrm{CDCl}_{3}\right) \delta 5.06(\mathrm{t}, J=7.4$ $\mathrm{Hz}, 1 \mathrm{H}), 4.73-4.68(\mathrm{~m}, 1 \mathrm{H}), 4.36(\mathrm{t}, J=5.8 \mathrm{~Hz}, 1 \mathrm{H}), 3.32(\mathrm{~s}, 6 \mathrm{H})$, 2.66-2.57 (m, $1 \mathrm{H}), 2.10-1.97$ (m, $2 \mathrm{H}), 1.84-1.77$ (m, $1 \mathrm{H}), 1.76-1.69$ (m, $1 \mathrm{H}), 1.64-1.58(\mathrm{~m}, 2 \mathrm{H}), 1.55-1.47(\mathrm{~m}, 1 \mathrm{H}), 1.42-1.22(\mathrm{~m}, 6 \mathrm{H})$, $1.19(\mathrm{~s}, 3 \mathrm{H}), 1.08-1.02(\mathrm{~m}, 21 \mathrm{H}), 0.85(\mathrm{~s}, 3 \mathrm{H}) ;{ }^{13} \mathrm{C}$ NMR $(125 \mathrm{MHz}$, $\left.\mathrm{CDCl}_{3}\right) \delta 141.9,122.8,105.0,67.6,53.1,48.2,42.9,33.9,32.9,31.3$, 30.4, 30.0, 28.5, 27.8, 24.8, 18.6, 18.5, 12.9; FT-IR (neat) 2943, $2865 \mathrm{~cm}^{-1}$; Anal. Calcd for $\mathrm{C}_{25} \mathrm{H}_{50} \mathrm{O}_{3} \mathrm{Si}$ : C, 70.36; H, 11.81; found: C, 70.59; H, 11.95 .

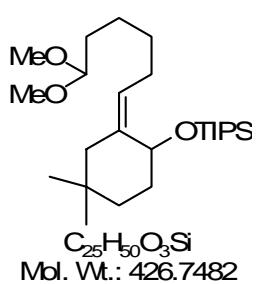

Z-[[6-(6,6-Dimethoxyhexylidene)-4,4-dimethylcyclohexyloxy] triisopropylsilane] (49). Following the general procedure, $0.81 \mathrm{~g} \mathrm{(72 \% )}$ of silyloxy 49 was isolated as a yellow oil: ${ }^{1} \mathrm{H}$ NMR $\left(500 \mathrm{MHz}, \mathrm{CDCl}_{3}\right)$ $\delta 5.00(\mathrm{t}, J=7.0 \mathrm{~Hz}, 1 \mathrm{H}), 4.66(\mathrm{br} \mathrm{s}, 1 \mathrm{H}), 4.36(\mathrm{t}, J=5.7 \mathrm{~Hz}, 1 \mathrm{H}), 3.32$ (s, $6 \mathrm{H}), 2.49(\mathrm{~d}, J=12.5 \mathrm{~Hz}, 1 \mathrm{H}), 2.46-2.11(\mathrm{~m}, 1 \mathrm{H}), 2.11-1.98(\mathrm{~m}, 1$ $\mathrm{H}), 1.85(\mathrm{td}, J=13.4,4 \mathrm{~Hz}, 1 \mathrm{H}), 1.72-1.69(\mathrm{~m}, 1 \mathrm{H}), 1.62-1.60(\mathrm{~m}, 2$ $\mathrm{H}), 1.5-1.53(\mathrm{~m}, 1 \mathrm{H}), 1.50-1.45(\mathrm{~m}, 1 \mathrm{H}), 1.40-1.31(\mathrm{~m}, 4 \mathrm{H}), 1.13-$ $1.00(\mathrm{~m}, 22 \mathrm{H}), 0.94(\mathrm{~s}, 3 \mathrm{H}), 0.79(\mathrm{~s}, 3 \mathrm{H}) ;{ }^{13} \mathrm{C} \mathrm{NMR}\left(125 \mathrm{MHz}, \mathrm{CDCl}_{3}\right) \delta 139.6,123.7$, 104.9, 65.6, 52.9, 45.8, 33.7, 32.7, 32.68, 32.63, 32.0, 30.26, 27.5, 24.6, 24.5, 18.4, 18.3, 18.2, 12.6; IR(neat) 2938, $2860 \mathrm{~cm}^{-1}$; HRMS (ESI) $\mathrm{m} / \mathrm{z} 449.3412(\mathrm{M}+\mathrm{Na}, 449.3427$ calcd for $\mathrm{C}_{25} \mathrm{H}_{50} \mathrm{O}_{3} \mathrm{SiNa}$ ). Anal. Calcd for $\mathrm{C}_{25} \mathrm{H}_{50} \mathrm{O}_{3} \mathrm{Si}$ : C, 70.36; H, 11.81; found: $\mathrm{C}$, $70.58 ; \mathrm{H}, 11.95$.

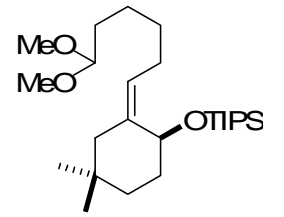

$\mathrm{C}_{25} \mathrm{H}_{50} \mathrm{O}_{3} \mathrm{Si}$ Mol. Wt.: 426.7482
(S)-Z-[[6-(6,6-Dimethoxyhexylidene)-4,4-dimethylcyclohexyloxy] triisopropylsilane] (49). Following the general procedure described above, $0.68 \mathrm{~g}(86 \%)$ of silyloxy $(S)-49$ was isolated: $[\alpha]^{23}{ }_{\mathrm{D}}+36.1$, $[\alpha]^{23}{ }_{577}+37.3,[\alpha]^{23}{ }_{546}+41.9,[\alpha]^{23}{ }_{435}+74.4,[\alpha]^{23}{ }_{405}+91.2$ (c 1.08 , $\mathrm{CHCl}_{3}$ ); all specta matched the racemate.

Prins-pinacol rearrangement of 21. Following the general procedure, silyloxy 21 ( 0.33 $\mathrm{g}, 0.82 \mathrm{mmol}$ ) provided the following fractions:

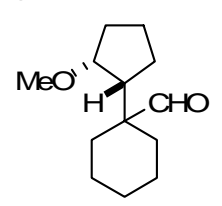

$\mathrm{C}_{13} \mathrm{H}_{22} \mathrm{O}_{2}$ Mol. W.: 210.312 51a, $52 \mathrm{mg}(29 \%)$ as a clear pale yellow oil: ${ }^{1} \mathrm{H}$ NMR $\left(500 \mathrm{MHz}, \mathrm{CDCl}_{3}\right)$ $\delta 9.63(\mathrm{~s}, 1 \mathrm{H}), 3.70-3.69(\mathrm{~m}, 1 \mathrm{H}), 3.18(\mathrm{~s}, 3 \mathrm{H}), 2.15-2.14(\mathrm{~m}, 1 \mathrm{H})$, 2.05-1.93 (m, $1 \mathrm{H}), 1.83-1.79(\mathrm{~m}, 2 \mathrm{H}), 1.73-1.64(\mathrm{~m}, 4 \mathrm{H}), 1.56-1.26$ (m, $5 \mathrm{H}), 1.24-1.18(\mathrm{~m}, 3 \mathrm{H}), 1.15-1.05(\mathrm{~m}, 1 \mathrm{H}) ;{ }^{13} \mathrm{C}$ NMR $(125 \mathrm{MHz}$, $\left.\mathrm{CDCl}_{3}\right) \delta 207.8,82.1,56.7,56.1,49.7,32.7,29.8,26.2,24.1,24.1,23.5$, for $\mathrm{C}_{13} \mathrm{H}_{22} \mathrm{O}_{2} \mathrm{Na}$ ). 


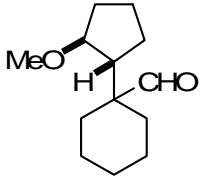

$\mathrm{C}_{13} \mathrm{H}_{22} \mathrm{O}_{2}$ Mol. W.: 210.3126

51b, $80 \mathrm{mg}(46 \%)$ as a clear pale yellow oil: ${ }^{1} \mathrm{H}$ NMR $\left(500 \mathrm{MHz}, \mathrm{CDCl}_{3}\right)$ $\delta 9.45(\mathrm{~s}, 1 \mathrm{H}), 3.56-3.53(\mathrm{~m}, 1 \mathrm{H}), 3.19(\mathrm{~s}, 3 \mathrm{H}), 1.94-1.91(\mathrm{~m}, 3 \mathrm{H})$, 1.69-1.53 (m, 8 H), 1.35-1.03 (m, $\left.6 \mathrm{H}) ;{ }^{13} \mathrm{C} \mathrm{NMR} \mathrm{(125} \mathrm{MHz,} \mathrm{CDCl}_{3}\right) \delta$ 207.5, 82.7, 56.8, 52.6, 51.3, 32.5, 30.4, 28.5, 25.9, 23.2, 22.8, 21.9, 17.2; IR(neat) $1722 \mathrm{~cm}^{-1}$; LRMS (ESI) $\mathrm{m} / \mathrm{z} 233(\mathrm{M}+\mathrm{Na}, 233$ calcd for $\left.\mathrm{C}_{13} \mathrm{H}_{22} \mathrm{O}_{2} \mathrm{Na}\right)$.

Prins-pinacol rearrangement of 22. Following the general procedure, silyloxy $22(0.26$ $\mathrm{g}, 0.64 \mathrm{mmol})$ provided the following fractions:

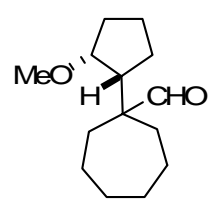

$\mathrm{C}_{14} \mathrm{H}_{24} \mathrm{O}_{2}$

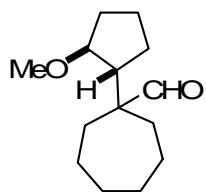

$\mathrm{C}_{14} \mathrm{H}_{24} \mathrm{O}_{2}$ Mol. Wt.: 224.3392 Mol. Wh: 224.3392

52a, $50 \mathrm{mg}(35 \%)$ as a clear pale yellow oil: ${ }^{1} \mathrm{H}$ NMR $\left(500 \mathrm{MHz}, \mathrm{CDCl}_{3}\right)$ $\delta 9.66(\mathrm{~s}, 1 \mathrm{H}), 3.72-3.70(\mathrm{~m}, 1 \mathrm{H}), 3.15(\mathrm{~s}, 3 \mathrm{H}), 2.19-2.15(\mathrm{~m}, 1 \mathrm{H})$, 1.96-1.67 (m, $6 \mathrm{H}), 1.60-1.35(\mathrm{~m}, 12 \mathrm{H}) ;{ }^{13} \mathrm{C} \mathrm{NMR}\left(125 \mathrm{MHz}, \mathrm{CDCl}_{3}\right) \delta$ 205.8, 82.3, 55.9, 54.9, 52.2, 33.4, 33.1, 31.0, 30.8, 29.8, 24.2, 23.3, 22.8, 22.2; IR(neat) $1718 \mathrm{~cm}^{-1}$; LRMS (ESI) $\mathrm{m} / \mathrm{z} 247$ (M+Na, 247 calcd for $\left.\mathrm{C}_{14} \mathrm{H}_{24} \mathrm{O}_{2} \mathrm{Na}\right)$.

52b, $52 \mathrm{mg}(36 \%)$ as a clear pale yellow oil: ${ }^{1} \mathrm{H}$ NMR $\left(500 \mathrm{MHz}, \mathrm{CDCl}_{3}\right)$ $\delta 9.48(\mathrm{~s}, 1 \mathrm{H}), 3.55-3.54(\mathrm{~m}, 1 \mathrm{H}), 3.26(\mathrm{~s}, 3 \mathrm{H}), 2.15-1.97(\mathrm{~m}, 2 \mathrm{H}), 1.95-$ $1.86(\mathrm{~m}, 1 \mathrm{H}), 1.83-1.70(\mathrm{~m}, 1 \mathrm{H}), 1.66-1.47(\mathrm{~m}, 12 \mathrm{H}), 1.33-1.26(\mathrm{~m}, 3$ $\mathrm{H}) ;{ }^{13} \mathrm{C}$ NMR $\left(125 \mathrm{MHz}, \mathrm{CDCl}_{3}\right) \delta 206.6,83.4,56.9,54.3,53.9,32.4$, $31.3,30.6,30.5,30.0,26.6,24.4,24.1,23.5$; IR(neat) $1725 \mathrm{~cm}^{-1}$; LRMS (ESI) $\mathrm{m} / \mathrm{z} 247\left(\mathrm{M}+\mathrm{Na}, 247\right.$ calcd for $\mathrm{C}_{14} \mathrm{H}_{24} \mathrm{O}_{2} \mathrm{Na}$ ).

Prins-pinacol rearrangement of 32. Following the general procedure, silyloxy $32(0.30$ $\mathrm{g}, 0.73 \mathrm{mmol})$ provided the following fractions:

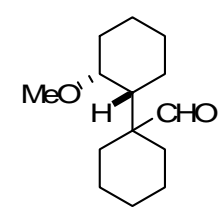

$\mathrm{C}_{14} \mathrm{H}_{24} \mathrm{O}_{2}$ Mol. Wt: 224.3392

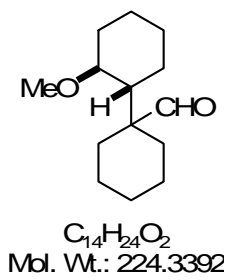

53a, $73 \mathrm{mg}(45 \%)$ as a clear pale yellow oil: ${ }^{1} \mathrm{H} \mathrm{NMR}\left(500 \mathrm{MHz}, \mathrm{CDCl}_{3}\right)$ $\delta 9.54(\mathrm{~s}, 1 \mathrm{H}), 3.58(\mathrm{bs}, 1 \mathrm{H}), 3.21$ (s, $3 \mathrm{H}), 2.15-2.00(\mathrm{~m}, 3 \mathrm{H}), 1.64-1.32$ $(\mathrm{m}, 11 \mathrm{H}), 1.21-1.13(\mathrm{~m}, 5 \mathrm{H}) ;{ }^{13} \mathrm{C} \mathrm{NMR}\left(125 \mathrm{MHz}, \mathrm{CDCl}_{3}\right) \delta 206.9$, $74.7,55.6,50.7,30.9,30.8,27.9,26.9,26.0,23.1,22.9,21.7,20.1$; IR(neat) $1710 \mathrm{~cm}^{-1}$; LRMS (ESI) $\mathrm{m} / \mathrm{z} 247(\mathrm{M}+\mathrm{Na}, 247$ calcd for $\left.\mathrm{C}_{14} \mathrm{H}_{24} \mathrm{O}_{2} \mathrm{Na}\right)$.

53b, $60 \mathrm{mg}(37 \%)$ as a clear pale yellow oil: ${ }^{1} \mathrm{H} \mathrm{NMR}\left(500 \mathrm{MHz}, \mathrm{CDCl}_{3}\right)$ $\delta 9.40(\mathrm{~s}, 1 \mathrm{H}), 3.20(\mathrm{~s}, 3 \mathrm{H}), 2.84(\mathrm{td}, J=10.3,4.2 \mathrm{~Hz}, 1 \mathrm{H}), 2.18-2.10$ $(\mathrm{m}, 1 \mathrm{H}), 1.84-1.44(\mathrm{~m}, 11 \mathrm{H}), 1.60-1.00(\mathrm{~m}, 6 \mathrm{H}) ;{ }^{13} \mathrm{C} \mathrm{NMR}(125 \mathrm{MHz}$, $\left.\mathrm{CDCl}_{3}\right) \delta 205.9,79.9,55.5,51.4,49.9,31.4,30.5,26.3,26.1,25.6,25.5$, 24.6, 23.1, 22.8; IR(neat) $1718 \mathrm{~cm}^{-1}$; LRMS (ESI) $\mathrm{m} / \mathrm{z} 247$ (M+Na, 247 calcd for $\mathrm{C}_{14} \mathrm{H}_{24} \mathrm{O}_{2} \mathrm{Na}$ ). 
Prins-pinacol rearrangement of 33. Following the general procedure, silyloxy $\mathbf{3 3}(0.30$ $\mathrm{g}, 0.70 \mathrm{mmol}$ ) provided the following fractions:
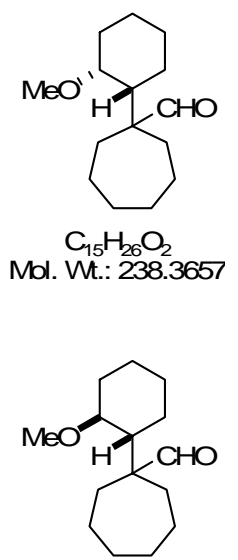

$\mathrm{C}_{15} \mathrm{H}_{26} \mathrm{O}_{2}$ Mol. W.: 238.365 $\mathrm{m} / \mathrm{z} 238.1939$

54a, $77 \mathrm{mg}(46 \%)$ as a clear pale yellow oil: ${ }^{1} \mathrm{H}$ NMR $\left(500 \mathrm{MHz}, \mathrm{CDCl}_{3}\right)$ $\delta 9.51(\mathrm{~s}, 1 \mathrm{H}), 3.53(\mathrm{bs}, 1 \mathrm{H}), 3.17(\mathrm{~s}, 3 \mathrm{H}), 2.10-2.00(\mathrm{~m}, 2 \mathrm{H}), 1.83-1.71$ (m, $2 \mathrm{H}), 1.64-1.43(\mathrm{~m}, 15 \mathrm{H}), 1.20-1.17(\mathrm{~m}, 2 \mathrm{H}) ;{ }^{13} \mathrm{C} \mathrm{NMR}(125 \mathrm{MHz}$, $\left.\mathrm{CDCl}_{3}\right) \delta 205.5,75.4,55.6,53.8,49.8,31.5,30.9,30.8,30.7,28.1,27.1$, 23.2, 22.8, 22.1, 20.1; IR(neat) $1715 \mathrm{~cm}^{-1}$; HRMS (CI) $\mathrm{m} / \mathrm{z} 238.1925$ (M, 238.1933 calcd for $\mathrm{C}_{15} \mathrm{H}_{26} \mathrm{O}_{2}$ ).

54b, $0.037 \mathrm{~g}(22 \%)$ as a clear pale yellow oil: ${ }^{1} \mathrm{H}$ NMR $(500 \mathrm{MHz}$, $\left.\mathrm{CDCl}_{3}\right) \delta 9.20(\mathrm{~s}, 1 \mathrm{H}), 3.13(\mathrm{~s}, 3 \mathrm{H}), 2.78(\mathrm{td}, J=10.5,4.4 \mathrm{~Hz}, 1 \mathrm{H})$, 2.18-2.10 (m, $1 \mathrm{H}), 2.10-2.05(\mathrm{~m}, 1 \mathrm{H}), 1.95-1.87(\mathrm{~m}, 1 \mathrm{H}), 1.78-1.63$ (m, $2 \mathrm{H}), 1.63-1.45(\mathrm{~m}, 10 \mathrm{H}), 1.43-1.17(\mathrm{~m}, 2 \mathrm{H}), 1.15-1.05(\mathrm{~m}, 4 \mathrm{H})$; ${ }^{13} \mathrm{C}$ NMR $\left(125 \mathrm{MHz}, \mathrm{CDCl}_{3}\right) \delta$ 203.4, 80.7, 55.7, 53.2, 52.5, 32.5, 31.5, 31.2, 26.7, 26.4, 25.8, 25.5, 24.7, 23.8; IR(neat) $1738 \mathrm{~cm}^{-1}$; HRMS (CI) (M, 238.1933 calcd for $\left.\mathrm{C}_{15} \mathrm{H}_{26} \mathrm{O}_{2}\right)$

Prins-pinacol rearrangement of 31. Following the general procedure, silyloxy $\mathbf{3 1}(0.30$ $\mathrm{g}, 0.79 \mathrm{mmol})$ provided the following fractions:

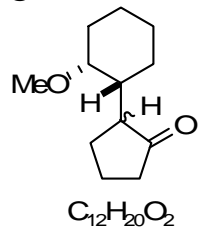

Mol. Wt: 196.2860

55, $76 \mathrm{mg}(50 \%)$ as a clear pale yellow oil: ${ }^{1} \mathrm{H}$ NMR $\left(500 \mathrm{MHz}, \mathrm{CDCl}_{3}\right) \delta$ 3.68 (bs, $1 \mathrm{H}), 3.30$ (s, $3 \mathrm{H}), 2.32-1.93$ (m, $6 \mathrm{H}), 1.85-1.53$ (m, 6H), 1.50$1.21(\mathrm{~m}, 4 \mathrm{H}) ;{ }^{13} \mathrm{C} \mathrm{NMR}\left(125 \mathrm{MHz}, \mathrm{CDCl}_{3}\right) \delta 222.0,78.4,56.6,52.5,41.9$, 39.0, 28.2, 27.9, 26.5, 24.7, 21.3, 20.2; IR(neat) $1738 \mathrm{~cm}^{-1}$; LRMS (ESI) $\mathrm{m} / \mathrm{z} 219\left(\mathrm{M}+\mathrm{Na}, 219\right.$ calcd for $\left.\mathrm{C}_{12} \mathrm{H}_{20} \mathrm{O}_{2}\right)$.

Prins-pinacol rearrangement of 47. Following the general procedure, silyloxy 47 (0.60 $\mathrm{g}, 1.4 \mathrm{mmol})$ provided the following fractions:

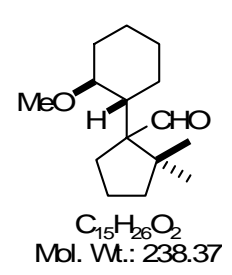

56, $25 \mathrm{mg}(8 \%):{ }^{1} \mathrm{H}$ NMR $\left(500 \mathrm{MHz}, \mathrm{CDCl}_{3}\right) \delta 9.89(\mathrm{~s}, 1 \mathrm{H}), 3.19(\mathrm{~s}, 3 \mathrm{H})$, 3.05-2.96 (m, $1 \mathrm{H}), 2.45-2.38(\mathrm{~m}, 1 \mathrm{H}), 2.27-2.25(\mathrm{~m}, 1 \mathrm{H}), 2.16-2.13(\mathrm{~m}$, $1 \mathrm{H}), 1.73-1.46(\mathrm{~m}, 14 \mathrm{H}), 1.22-0.89(\mathrm{~m}, 8 \mathrm{H}) ;{ }^{13} \mathrm{C}$ NMR $(125 \mathrm{MHz}$, $\left.\mathrm{CDCl}_{3}\right) \delta 208.3,81.8,62.5,55.7,50.4,46.0,42.6,34.0,31.8,28.4,26.6$, 25.2, 25.0, 24.6, 20.4; IR(neat) $1718 \mathrm{~cm}^{-1}$; HRMS (APCI) $\mathrm{m} / \mathrm{z} 261.1827$ $\left(\mathrm{M}+\mathrm{Na}, 261.1830\right.$ calcd for $\left.\mathrm{C}_{15} \mathrm{H}_{26} \mathrm{O}_{2} \mathrm{Na}\right)$.

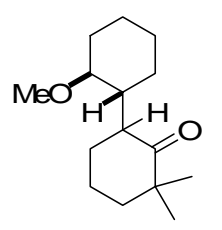

57, $69 \mathrm{mg},(21 \%):{ }^{1} \mathrm{H}$ NMR $\left(500 \mathrm{MHz}, \mathrm{CDCl}_{3}\right): 3.33$ (td, $J=9.5,3.9 \mathrm{~Hz}$, $1 \mathrm{H}), 2.96$ (ddd, $J=13.4,5.4,2.7 \mathrm{~Hz}, 1 \mathrm{H}), 2.16-2.12(\mathrm{~m}, 1 \mathrm{H}), 1.88-1.77$ (m, $2 \mathrm{H}), 1.75-1.62(\mathrm{~m}, 4 \mathrm{H}), 1.61-1.50(\mathrm{~m}, 5 \mathrm{H}), 1.31-1.21(\mathrm{~m}, 2 \mathrm{H})$, $1.16(\mathrm{~s}, 3 \mathrm{H}), 1.13-1.09(\mathrm{~m}, 1 \mathrm{H}), 1.02(\mathrm{~s}, 3 \mathrm{H}) ;{ }^{13} \mathrm{C}$ NMR (125 MHz, $\left.\mathrm{CDCl}_{3}\right) \delta 217.2,80.9,56.6,46.1,46.0,45.4,41.5,31.7,31.6,26.7,26.6$, Mol. Wt: 238.3651 26.4, 26.2, 25.2, 22.3; IR(neat) $1732 \mathrm{~cm}^{-1}$; HRMS (ESI) $\mathrm{m} / \mathrm{z} 261.1824$ $\left(\mathrm{M}+\mathrm{Na}, 261.1830\right.$ calcd for $\left.\mathrm{C}_{15} \mathrm{H}_{25} \mathrm{O}_{2} \mathrm{Na}\right)$. 
Prins-pinacol rearrangement of 48. Following the general procedure, silyloxy 48 (1.0 $\mathrm{g}, 2.35 \mathrm{mmol})$ provided the following fractions:

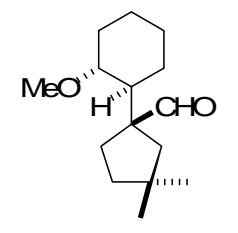

$\mathrm{C}_{15} \mathrm{H}_{26} \mathrm{O}_{2}$ Mol. Wt.: 238.3657

58a, $178 \mathrm{mg}(30 \%):{ }^{1} \mathrm{H}$ NMR $\left(500 \mathrm{MHz}, \mathrm{CDCl}_{3}\right) \delta 9.4(\mathrm{~s}, 1 \mathrm{H}), 3.16(\mathrm{~s}$, $3 \mathrm{H}), 2.66(\mathrm{td}, J=10.3,4.4 \mathrm{~Hz}, 1 \mathrm{H}), 2.14-2.10(\mathrm{~m}, 1 \mathrm{H}), 2.00(\mathrm{~d}, J=13$ $\mathrm{Hz}, 1 \mathrm{H}), 1.89-1.85(\mathrm{~m}, 1 \mathrm{H}), 1.81-1.76(\mathrm{~m}, 4 \mathrm{H}), 1.54-1.50(\mathrm{~m}, 1 \mathrm{H})$, 1.31-1.22 (m, $5 \mathrm{H}), 1.10-1.06(\mathrm{~m}, 2 \mathrm{H}), 1.04(\mathrm{~s}, 3 \mathrm{H}), 1.02(\mathrm{~s}, 3 \mathrm{H}) ;{ }^{13} \mathrm{C}$ NMR $\left(125 \mathrm{MHz}, \mathrm{CDCl}_{3}\right) \delta 201.5,82.0,59.9,55.6,47.8,39.6,39.4,38.7$, $31.3,30.03,30.00,28.7,25.8,25.4,24.5$; IR(neat) $1718 \mathrm{~cm}^{-1}$; HRMS (CI) $\mathrm{m} / \mathrm{z} 239.2022\left(\mathrm{M}+\mathrm{H}, 239.2011\right.$ calcd for $\left.\mathrm{C}_{15} \mathrm{H}_{27} \mathrm{O}_{2}\right)$.

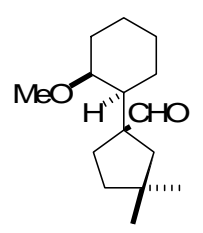

$\mathrm{C}_{15} \mathrm{H}_{26} \mathrm{O}$ Mol. Wt: 238.365

58b, $100 \mathrm{mg}(18 \%):{ }^{1} \mathrm{H} \mathrm{NMR}\left(500 \mathrm{MHz}, \mathrm{CDCl}_{3}\right) \delta 9.57(\mathrm{~s}, 1 \mathrm{H}), 3.45$ (bs, $1 \mathrm{H}), 3.18(\mathrm{~s}, 3 \mathrm{H}), 2.29-2.22(\mathrm{~m}, 1 \mathrm{H}), 2.05-1.98(\mathrm{~m}, 1 \mathrm{H}), 1.95(\mathrm{~d}$, $J=13.6,1 \mathrm{H}), 1.60-1.05(\mathrm{~m}, 12 \mathrm{H}), 1.0(\mathrm{~s}, 3 \mathrm{H}), 0.92(\mathrm{~s}, 3 \mathrm{H}) ;{ }^{13} \mathrm{C} \mathrm{NMR}$ $\left(125 \mathrm{MHz}, \mathrm{CDCl}_{3}\right) \delta$ 205.1, 77.0, 61.2, 56.1, 54.5, 47.5, 41.0, 39.1, 32.0, 31.1, 29.9, 28.3, 27.2, 23.7, 20.4; IR(neat) $1718 \mathrm{~cm}^{-1}$; HRMS (CI) $\mathrm{m} / \mathrm{z}$ 239.2013 (M+H, 239.2011 calcd for $\left.\mathrm{C}_{15} \mathrm{H}_{27} \mathrm{O}_{2}\right)$.

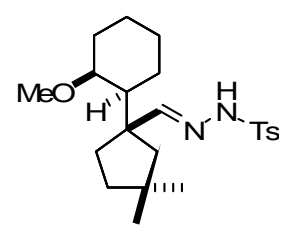

[1-(2-Methoxycyclohexyl)-2,2-dimethylcyclopentylmethylene] ptoluene sulfonate (59). A solution of $\mathbf{5 8 b}(90 \mathrm{mg}, 0.38 \mathrm{mmol})$, glacial acetic acid $(2.0 \mathrm{~mL})$ and tosyl hydrazone $(0.14 \mathrm{~g}, 0.75 \mathrm{mmol})$ was maintained at room temperature for $18 \mathrm{~h}$. The reaction mixture was diluted with heptane $(5 \mathrm{~mL})$, concentrated, and the residue was purified by flash column chromatography on silica gel $(20 \%$ ethyl acetate hexanes) to give $77 \mathrm{mg}(50 \%)$ of 59 as an off-white solid: ${ }^{1} \mathrm{H}$ NMR Mol. Wt: 406.5820 $\left(500 \mathrm{MHz}, \mathrm{CDCl}_{3}\right) \delta 7.81(\mathrm{~d}, J=8.3 \mathrm{~Hz}, 1 \mathrm{H}), 7.32(\mathrm{~d}, J=8.8 \mathrm{~Hz}, 1 \mathrm{H}), 7.22(\mathrm{~s}, 1 \mathrm{H})$, $3.41(\mathrm{~s}, 1 \mathrm{H}), 3.07(\mathrm{~s}, 3 \mathrm{H}), 2.41(\mathrm{~s}, 3 \mathrm{H}), 2.00(\mathrm{~d}, J=13.5 \mathrm{~Hz}, 1 \mathrm{H}), 1.96(\mathrm{~d}, J=14 \mathrm{~Hz}, 1$ $\mathrm{H}), 1.88-1.83(\mathrm{~m}, 1 \mathrm{H}), 1.62-1.56(\mathrm{~m}, 3 \mathrm{H}), 1.33-1.05(\mathrm{~m}, 10 \mathrm{H}), 0.93(\mathrm{~s}, 3 \mathrm{H}), 0.83(\mathrm{~s}, 3$ $\mathrm{H}) ;{ }^{13} \mathrm{C}$ NMR $\left(125 \mathrm{MHz}, \mathrm{CDCl}_{3}\right) \delta 159.1,143.9,135.8,129.6,128.4,77.0,55.7,54.2$, 54.0, 49.1, 39.9, 38.4, 34.7, 31.6, 30.1, 28.3, 26.7, 23.6, 21.7, 20.1; IR(neat) 3212, 1711 $\mathrm{cm}^{-1}$; HRMS (APCI) m/z $429.2200\left(\mathrm{M}+\mathrm{Na}, 429.2188\right.$ calcd for $\mathrm{C}_{22} \mathrm{H}_{34} \mathrm{~N}_{2} \mathrm{O}_{3} \mathrm{SNa}$ ).

Prins-pinacol rearrangement of 49. Following the general procedure described above, silyloxy 49 (0.20 g, $0.47 \mathrm{mmol})$ yielded the following fractions:

60a, $39 \mathrm{mg}(34 \%)$ as a clear oil: ${ }^{1} \mathrm{H}$ NMR $\left(500 \mathrm{MHz}, \mathrm{CDCl}_{3}\right) \delta 9.58(\mathrm{~s}, 1$

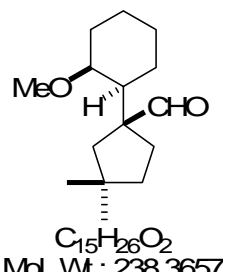
H), 3.34 (br s, $1 \mathrm{H}), 3.14$ (s, $3 \mathrm{H}), 2.22(\mathrm{~d}, 1 \mathrm{H}), 2.03-1.98(\mathrm{~m}, 2 \mathrm{H}), 1.80$ $1.77(\mathrm{~m}, 1 \mathrm{H}), 1.75-1.65(\mathrm{~m}, 1 \mathrm{H}), 1.64-1.60(\mathrm{~m}, 2 \mathrm{H}), 1.50-1.38(\mathrm{~m}, 3$ $\mathrm{H}), 1.35-1.30(\mathrm{~m}, 1 \mathrm{H}), 1.25-1.17(\mathrm{~m}, 4 \mathrm{H}), 1.02(\mathrm{~s}, 3 \mathrm{H}), 1.01(\mathrm{~s}, 3 \mathrm{H})$; ${ }^{13} \mathrm{C} \mathrm{NMR}\left(125 \mathrm{MHz}, \mathrm{CDCl}_{3}\right) \delta 203.6,77.1,61.23,55.8,54.2,45.6,39.6$, 39.1, 32.2, 30.9, 29.4, 28.1, 26.9, 23.2, 20.2; IR(neat) $1704 \mathrm{~cm}^{-1}$; HRMS (EI) $m / z 238.1932\left(\mathrm{M}, 238.1933\right.$ calcd for $\left.\mathrm{C}_{15} \mathrm{H}_{26} \mathrm{O}_{2}\right)$.

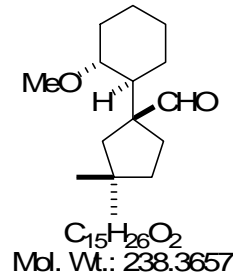

60b, $55 \mathrm{mg}(50 \%)$ as a clear oil: ${ }^{1} \mathrm{H} \mathrm{NMR}\left(500 \mathrm{MHz}, \mathrm{CDCl}_{3}\right) \delta 9.31(\mathrm{~s}, 1$ H), $3.13(\mathrm{~s}, 3 \mathrm{H}), 2.72(\mathrm{td}, J=10.45,4.3 \mathrm{~Hz}, 1 \mathrm{H}), 2.20-2.08(\mathrm{~m}, 2 \mathrm{H})$, 1.92-1.85 (m, $1 \mathrm{H}), 1.82-1.75(\mathrm{~m}, 2 \mathrm{H}), 1.72-1.65(\mathrm{~m}, 1 \mathrm{H}), 1.52-1.45$ (m, $5 \mathrm{H}), 1.20-1.10(\mathrm{~m}, 2 \mathrm{H}), 1.10-1.05(\mathrm{~m}, 2 \mathrm{H}), 1.00(\mathrm{~s}, 3 \mathrm{H}), 0.94(\mathrm{~s}, 3$ $\mathrm{H}) ;{ }^{13} \mathrm{C}$ NMR $\left(125 \mathrm{MHz}, \mathrm{CDCl}_{3}\right) \delta 201.6,82.3,59.8,55.7,48.8,44.6$, 
42.1, 39.2, 31.6, 29.9, 29.4, 26.2, 25.4, 24.9, 17.9; IR(neat) $1718 \mathrm{~cm}^{-1}$; HRMS (EI) $\mathrm{m} / \mathrm{z}$ 238.1936 (M, 238.1933 calcd for $\mathrm{C}_{15} \mathrm{H}_{26} \mathrm{O}_{2}$ ).

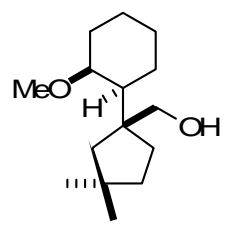

72

$\mathrm{C}_{15} \mathrm{H}_{28} \mathrm{O}_{2}$ Mol. Wt: 240.3816

\section{[1-(2-Methoxycyclohexyl)-3,3-dimethylcyclopentyl]methanol}

(72). $\mathrm{NaBH}_{4}(7 \mathrm{mg}, 0.2 \mathrm{mmol})$ was added in one portion to 60a $(48 \mathrm{mg}, 0.2$ $\mathrm{mmol})$ in $\mathrm{MeOH}(2 \mathrm{~mL})$ at $0{ }^{\circ} \mathrm{C}$. After stirring for $20 \mathrm{~min}$, water $(5 \mathrm{~mL})$ and $\mathrm{Et}_{2} \mathrm{O}(5 \mathrm{~mL})$ were added, and the organic layer was washed with brine $(10 \mathrm{~mL})$, dried $\left(\mathrm{MgSO}_{4}\right)$, and concentrated. The resulting clear oil (48 $\mathrm{mg}$, $100 \%)$ was used without further purification: ${ }^{1} \mathrm{H}$ NMR $\left(500 \mathrm{MHz}, \mathrm{CDCl}_{3}\right)$ H), $3.31(\mathrm{~s}, 3 \mathrm{H}), 3.09$ (t, $J=10.8 \mathrm{~Hz}, 1 \mathrm{H}), 2.12(\mathrm{~d}, J=14.2 \mathrm{~Hz}, 1 \mathrm{H}), 1.82-1.77$ (m, 2H), 1.62-1.55 (m, 1H), 1.50-1.39 (m, $7 \mathrm{H}), 1.33-.20(\mathrm{~m}, 4 \mathrm{H}), 1.08$ (s, 3H), $1.00(\mathrm{~s}$, $3 \mathrm{H}) ;{ }^{13} \mathrm{C}$ NMR $\left(125 \mathrm{MHz}, \mathrm{CDCl}_{3}\right) \delta 152.3,77.1,66.1,55.2,54.3,46.2,39.9,39.1,37.1$, 31.2, 30.9, 28.3, 27.1, 23.2, 19.9; IR(neat) $3404 \mathrm{~cm}^{-1}$; HRMS (ESI) $\mathrm{m} / \mathrm{z} 263.1991$ $\left(\mathrm{M}+\mathrm{Na}, 263.1987\right.$ calcd for $\left.\mathrm{C}_{15} \mathrm{H}_{28} \mathrm{O}_{2} \mathrm{Na}\right)$.

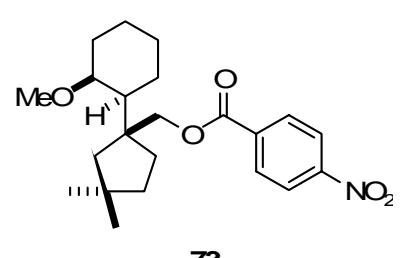

4-Nitrobenzoic acid 1-(2-methoxy-cyclohexyl)-3,3dimethylcyclopentyl methylester (73). 4-nitrobenoyl chloride $(13 \mathrm{mg}, 0.08 \mathrm{mmol})$ was added to a solution of alcohol $72(10$ $\mathrm{mg}, 0.04 \mathrm{mmol})$ and triethylamine $(20 \mathrm{uL}, 0.16 \mathrm{mmol})$ in $\mathrm{CH}_{2} \mathrm{Cl}_{2}$ $(0.4 \mathrm{~mL})$ at room temperature. After stirring for $18 \mathrm{~h}$, the reaction was partitioned between $\mathrm{CH}_{2} \mathrm{Cl}_{2}(5 \mathrm{~mL})$ and $\mathrm{H}_{2} \mathrm{O}(5$

$\mathrm{C}_{22} \mathrm{H}_{31} \mathrm{NO}_{5}$ Mol. Wt: 389.4852 $\mathrm{mL}$ ) and the layers were separated. The combined organic layers were washed with brine $(10 \mathrm{~mL})$, dried $\left(\mathrm{Na}_{2} \mathrm{SO}_{4}\right)$, filtered and concentrated in vacuo. The residue was purified by flash column chromatography on silica gel ( $2 \%$ ethyl acetate hexanes) to yield $12 \mathrm{mg}(80 \%)$ of the benzoate as a colorless oil: ${ }^{1} \mathrm{H}$ NMR (500 $\mathrm{MHz}$, $\left.\mathrm{CDCl}_{3}\right) \delta 8.31(\mathrm{~d}, J=8.6 \mathrm{~Hz}, 2 \mathrm{H}), 8.20(\mathrm{~d}, J=8.6 \mathrm{~Hz}, 1 \mathrm{H}), 4.43(\mathrm{~d}, J=11 \mathrm{~Hz}, 1 \mathrm{H})$, $4.29(\mathrm{~d}, J=11 \mathrm{~Hz}, 1 \mathrm{H}), 3.58(\mathrm{bs}, 1 \mathrm{H}), 3.25(\mathrm{~s}, 3 \mathrm{H}), 2.08(\mathrm{~d}, J=14 \mathrm{~Hz}, 1 \mathrm{H}), 1.86-$ $1.68(\mathrm{~m}, 4 \mathrm{H}), 1.65-1.61(\mathrm{~m}, 2 \mathrm{H}), 1.56-1.40(\mathrm{~m}, 6 \mathrm{H}), 1.25-1.10(\mathrm{~m}, 2 \mathrm{H}), 1.07$ (s, 3H), $1.04(\mathrm{~s}, 3 \mathrm{H}) ;{ }^{13} \mathrm{C}$ NMR $\left(125 \mathrm{MHz}, \mathrm{CDCl}_{3}\right) \delta 164.9,150.5,136.2,130.7,123.6,77.0$, 70.7, 55.5, 49.6, 49.5, 48.3, 40.7, 38.6, 32.9, 30.8, 28.6, 27.2, 23.1, 20.1; IR(neat) 1720, $1530 \mathrm{~cm}^{-1}$; HRMS (ESI) $\mathrm{m} / \mathrm{z} 412.2117\left(\mathrm{M}+\mathrm{Na}, 412.2100\right.$ calcd for $\mathrm{C}_{22} \mathrm{H}_{31} \mathrm{O}_{5} \mathrm{NNa}$ ).

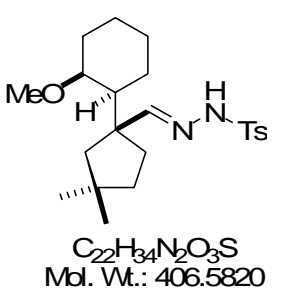

[1-(2-Methoxycyclohexyl)-3,3-dimethylcyclopentylmethylene]ptoluenesulfonate (61). A solution of 60a $(20 \mathrm{mg}, 0.08 \mathrm{mmol})$, glacial acetic acid $(2 \mathrm{~mL})$ and tosyl hydrazone $(0.36 \mathrm{~g}, 0.16 \mathrm{mmol})$ was maintained at room temperature for $18 \mathrm{~h}$. The reaction mixture was diluted with heptane $(5 \mathrm{~mL})$, concentrated, and the residue was purified by flash column chromatography on silica gel $(20 \%$ ethyl acetate hexanes) to give $28 \mathrm{mg}(84 \%)$ of $\mathbf{6 0 a}$ as an off-white solid: ${ }^{1} \mathrm{H}$ NMR $\left(500 \mathrm{MHz}, \mathrm{CDCl}_{3}\right) \delta 7.83(\mathrm{~d}, J=8.3 \mathrm{~Hz}, 2 \mathrm{H}), 7.32(\mathrm{~d}, J=8.3 \mathrm{~Hz}, 2 \mathrm{H}), 7.16(\mathrm{~s}, 1 \mathrm{H})$, 3.27 (br s, 1 H), 2.93 (s, 3 H), 2.40 (s, $3 \mathrm{H}), 2.00-1.80$ (m, $3 \mathrm{H}), 1.61-1.58(\mathrm{~m}, 1 \mathrm{H}), 1.5-$ $1.4(\mathrm{~m}, 1 \mathrm{H}), 1.37-1.30(\mathrm{~m}, 3 \mathrm{H}), 1.30-1.20(\mathrm{~m}, 4 \mathrm{H}), 1.20-1.05(\mathrm{~m}, 3 \mathrm{H}), 0.95(\mathrm{~s}, 3 \mathrm{H})$, 0.89 (s, $3 \mathrm{H}) ;{ }^{13} \mathrm{C}$ NMR $\left(125 \mathrm{MHz}, \mathrm{CDCl}_{3}\right) \delta 158.7,143.9,135.8,129.6,128.4,77.3$, 55.6, 54.3, 54.0, 49.0, 39.5, 38.4, 35.0, 31.7, 29.8, 28.4, 26.7, 23.2, 21.7, 20.0; IR(neat) 
3220, $1695 \mathrm{~cm}^{-1}$; HRMS (ESI) $\mathrm{m} / \mathrm{z} \quad 429.2192 \quad(\mathrm{M}+\mathrm{Na}, 429.2188$ calcd for $\mathrm{C}_{22} \mathrm{H}_{34} \mathrm{O}_{3} \mathrm{~N}_{2} \mathrm{SNa}$ ).

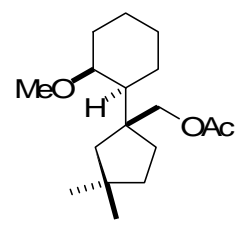

$\mathrm{C}_{77} \mathrm{H}_{30} \mathrm{O}_{3}$ Mol. Wt: : 282.41830

Acetic acid 1-(2-methoxycyclohexyl)-3,3-dimethylcyclopentylmethyl ester (62). $\mathrm{NaBH}_{4}(8.0 \mathrm{mg}, 0.21 \mathrm{mmol})$ was added portionwise to a solution of aldehyde 60a $(25 \mathrm{mg}, 0.1 \mathrm{mmol})$ and $\mathrm{MeOH}(2 \mathrm{~mL})$ at $0{ }^{\circ} \mathrm{C}$. After stirring for $20 \mathrm{~min}$, water $(5 \mathrm{~mL})$ and $\mathrm{Et}_{2} \mathrm{O}(5 \mathrm{~mL})$ were added, and the organic layer was washed with brine $(20 \mathrm{~mL})$, dried $\left(\mathrm{MgSO}_{4}\right)$ and concentrated. The residue was dissolved in pyridine $(2 \mathrm{~mL})$ followed by the addition of $\mathrm{Ac}_{2} \mathrm{O}(40 \mu \mathrm{L}, 0.4 \mathrm{mmol})$. After stirring for $18 \mathrm{~h}$, the reaction mixture was partitioned between pentane $(5 \mathrm{~mL})$ and water $(5 \mathrm{~mL})$, and the organic layer was sequentially washed with saturated aqueous $\mathrm{CuSO}_{4}(3 \times 10 \mathrm{~mL})$, water $(10 \mathrm{~mL})$, brine $(10 \mathrm{~mL})$, dried $\left(\mathrm{MgSO}_{4}\right)$ and concentrated. The residue was chromatographed on silica gel (2\% ethyl acetate-hexanes) to yield $20 \mathrm{mg}(67 \%)$ of $\mathbf{6 2}$ as a pale yellow oil: ${ }^{1} \mathrm{H}$ NMR $\left(500 \mathrm{MHz}, \mathrm{CDCl}_{3}\right) \delta 4.03(\mathrm{~d}, J=11.4 \mathrm{~Hz}, 1 \mathrm{H}), 3.99(\mathrm{~d}, J=$ $11.4 \mathrm{~Hz}, 1 \mathrm{H}), 3.51-3.49$ (m, $1 \mathrm{H}), 3.22$ (s, $3 \mathrm{H}), 2.10-2.07$ (m, $1 \mathrm{H}), 2.05$ (s, $3 \mathrm{H}), 1.60$ $1.05(\mathrm{~m}, 12 \mathrm{H}), 1.03(\mathrm{~s}, 3 \mathrm{H}), 1.00(\mathrm{~s}, 3 \mathrm{H}) ;{ }^{13} \mathrm{C} \mathrm{NMR}\left(125 \mathrm{MHz}, \mathrm{CDCl}_{3}\right) \delta$ 171.6, 76.6, 69.6, 55.4, 49.1, 48.5, 47.9, 40.9, 38.5, 32.5, 30.8, 30.7, 28.5, 27.1, 22.7, 21.2, 20.2; IR(neat) 1741, $1239 \mathrm{~cm}^{-1}$; HRMS (ESI) $\mathrm{m} / z 305.2094(\mathrm{M}+\mathrm{Na}, 305.2093$ calcd for $\left.\mathrm{C}_{17} \mathrm{H}_{30} \mathrm{O}_{3} \mathrm{NNa}\right)$.

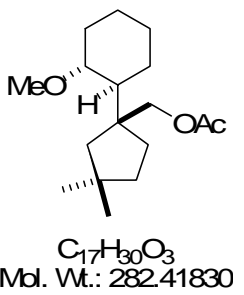

Acetic acid 1-(2-methoxy-cyclohexyl)-3,3-dimethylcyclopentylmethyl ester (63). Following the procedure above, aldehyde 60b (10 mg, 0.04 mmol), provided $10 \mathrm{mg}(85 \%)$ of $\mathbf{6 3}$ as a pale yellow oil: ${ }^{1} \mathrm{H}$ NMR $(500$ $\left.\mathrm{MHz}, \mathrm{CDCl}_{3}\right) \delta 4.01(\mathrm{~d}, J=11.3 \mathrm{~Hz}, 1 \mathrm{H}), 3.92(\mathrm{~d}, J=11.3 \mathrm{~Hz}, 1 \mathrm{H})$, $3.29(\mathrm{~s}, 3 \mathrm{H}), 2.96(\mathrm{td}, J=10.3,4.0 \mathrm{~Hz}, 1 \mathrm{H}), 2.24-2.18(\mathrm{~m}, 1 \mathrm{H}), 2.06$ $(\mathrm{s}, 3 \mathrm{H}), 1.87-1.82(\mathrm{~m}, 1 \mathrm{H}), 1.78-1.55(\mathrm{~m}, 6 \mathrm{H}), 1.50-1.30(\mathrm{~m}, 4 \mathrm{H})$, $1.2-1.0(\mathrm{~m}, 6 \mathrm{H}), 0.97(\mathrm{~s}, 3 \mathrm{H}) ;{ }^{13} \mathrm{C}$ NMR $\left(125 \mathrm{MHz}, \mathrm{CDCl}_{3}\right) \delta 171.4$, 82.4, 67.7, 55.5, 54.1, 52.5, 49.0, 39.1, 38.5, 34.1, 31.10, 31.12, 28.6, 26.9, 25.1, 21.3; IR(neat) 1733, $1241 \mathrm{~cm}^{-1}$; HRMS (ESI) $\mathrm{m} / \mathrm{z} 305.2094(\mathrm{M}+\mathrm{Na}, 305.2093$ calcd for $\left.\mathrm{C}_{17} \mathrm{H}_{30} \mathrm{O}_{3} \mathrm{Na}\right)$.

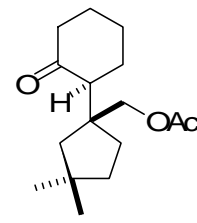

$\mathrm{C}_{16} \mathrm{H}_{26} \mathrm{O}_{3}$ Mol. Wt.: 266.37584

Acetic acid 3,3-dimethyl-1-(2-oxocyclohexyl)cyclopentylmethyl ester (64). Sodium periodate $(72 \mathrm{mg}, 0.34 \mathrm{mmol})$ was added to a mixture of 63 $(24 \mathrm{mg}, 0.08 \mathrm{mmol})$ in $\mathrm{MeCN}(1.0 \mathrm{~mL}), \mathrm{CCl}_{4}(1.0 \mathrm{~mL})$, and $\mathrm{pH} 7$ aqueous phosphate buffer $(1.5 \mathrm{~mL})$. After stirring for $20 \mathrm{~min}$, $\mathrm{RuCl}_{3} \cdot \mathrm{xH}_{2} \mathrm{O}(2.0 \mathrm{mg}, 0.008 \mathrm{mmol})$ was added and the reaction was vigorously stirred for $18 \mathrm{~h}$. The crude reaction mixture was filtered through a pad of Florisil ${ }^{\circledR}$ and concentrated. The residue was absorbed onto silica gel and chromatographed (2\% ethyl acetate - hexanes) to yield $15 \mathrm{mg}(71 \%)$ of 64 as a colorless oil: ${ }^{1} \mathrm{H}$ NMR $\left(500 \mathrm{MHz}, \mathrm{CDCl}_{3}\right) \delta 4.21(\mathrm{~d}, J=10.9 \mathrm{~Hz}, 1 \mathrm{H}), 4.14(\mathrm{~d}$, $J=10.9 \mathrm{~Hz}, 1 \mathrm{H}), 4.49-4.44(\mathrm{~m}, 1 \mathrm{H}), 2.35-2.25(\mathrm{~m}, 2 \mathrm{H}), 2.20-2.14(\mathrm{~m}, 1 \mathrm{H}), 2.11-$ $2.05(\mathrm{~m}, 1 \mathrm{H}), 2.04(\mathrm{~s}, 3 \mathrm{H}), 1.97-1.90(\mathrm{~m}, 1 \mathrm{H}), 1.77-1.70(\mathrm{~m}, 1 \mathrm{H}), 1.70-1.55(\mathrm{~m}, 5 \mathrm{H})$, 1.54-1.47 (m, $1 \mathrm{H}), 1.42-1.35$ (m, $2 \mathrm{H}), 1.04$ (s, $3 \mathrm{H}), 0.99$ (s, $3 \mathrm{H}) ;{ }^{13} \mathrm{C}$ NMR $(125 \mathrm{MHz}$, $\left.\mathrm{CDCl}_{3}\right) \delta 212.3,171.2,68.7,59.4,49.6,47.3,43.8,40.3,39.1,33.4,30.8,30.6,30.3$, 
28.4, 26.5, 21.2; IR(neat) 1738, 1708, $1236 \mathrm{~cm}^{-1}$; HRMS (ESI) $\mathrm{m} / \mathrm{z} 289.1777(\mathrm{M}+\mathrm{Na}$, 289.1780 calcd $\left.\mathrm{C}_{17} \mathrm{H}_{26} \mathrm{O}_{3} \mathrm{Na}\right)$.

Prins-pinacol rearrangement of (S)-49. Following the general procedure, silyloxy (S)$49(0.20 \mathrm{~g}, 0.47 \mathrm{mmol})$ provided the following fractions:

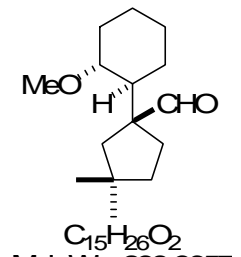

$\left(1 S, 1^{\prime} S, 2^{\prime} S\right)-60 a, 55 \mathrm{mg}(50 \%):[\alpha]^{23}-35.9,[\alpha]^{23}{ }_{577}-38.1,[\alpha]^{23}{ }_{546}$

$-43.2,[\alpha]^{23}{ }_{435}-71.9,[\alpha]^{23}{ }_{405}-88.1\left(\mathrm{c} 1.13, \mathrm{CHCl}_{3}\right)$; all specta matched the racemate.

Mol. Wt: 238.3657

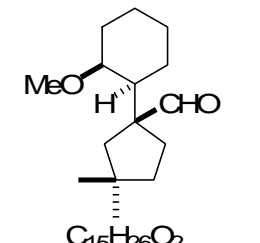

$\left(1 S, 1^{\prime} S, 2^{\prime} R\right)-60 b, 36 \mathrm{mg}(32 \%):[\alpha]^{23}{ }_{\mathrm{D}}-31.7,[\alpha]^{23}{ }_{577}-32.4,[\alpha]^{23}{ }_{546}$ $-36.1,[\alpha]^{23}{ }_{435}-59.9,[\alpha]^{23}{ }_{405}-72.2\left(\mathrm{c} 1.13, \mathrm{CHCl}_{3}\right)$; all specta matched the racemate.

Mol. Wt: 238.3657

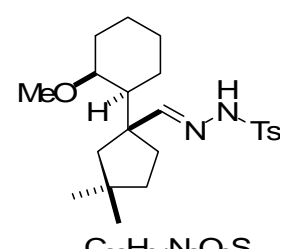

Tosyl Hydrazone (1S,1'S,2'S)-61. A solution of ( $1 S, 1^{\prime} S, 2$ 'S)-60a (55 $\mathrm{mg}, 0.23 \mathrm{mmol})$, glacial acetic acid $(2.0 \mathrm{~mL})$ and tosyl hydrazone $(86$ $\mathrm{mg}, 0.46 \mathrm{mmol}$ ) was maintained at room temperature for $18 \mathrm{~h}$. The reaction mixture was diluted with heptane $(5 \mathrm{~mL})$, concentrated, and the residue was purified by flash column chromatography on silica gel Mol. Wt.: 406.5820 (20\% ethyl acetate - hexanes) to give $76 \mathrm{mg}(81 \%)$ of (1S, 1'S, 2'S)-61 as an off-white solid: $[\alpha]^{23}{ }_{D}+32.9,[\alpha]^{23}{ }_{577}+25.9,[\alpha]^{23}{ }_{546}+26.8$, $[\alpha]^{23}{ }_{435}+23.1,[\alpha]^{23}{ }_{405}+16.4\left(\mathrm{c} 0.07, \mathrm{CH}_{2} \mathrm{Cl}_{2}\right)$; all specta matched the racemate. 
B. Copies of ${ }^{1} \mathrm{H}$ and ${ }^{13} \mathrm{C}$ NMR spectra for all new compounds: 

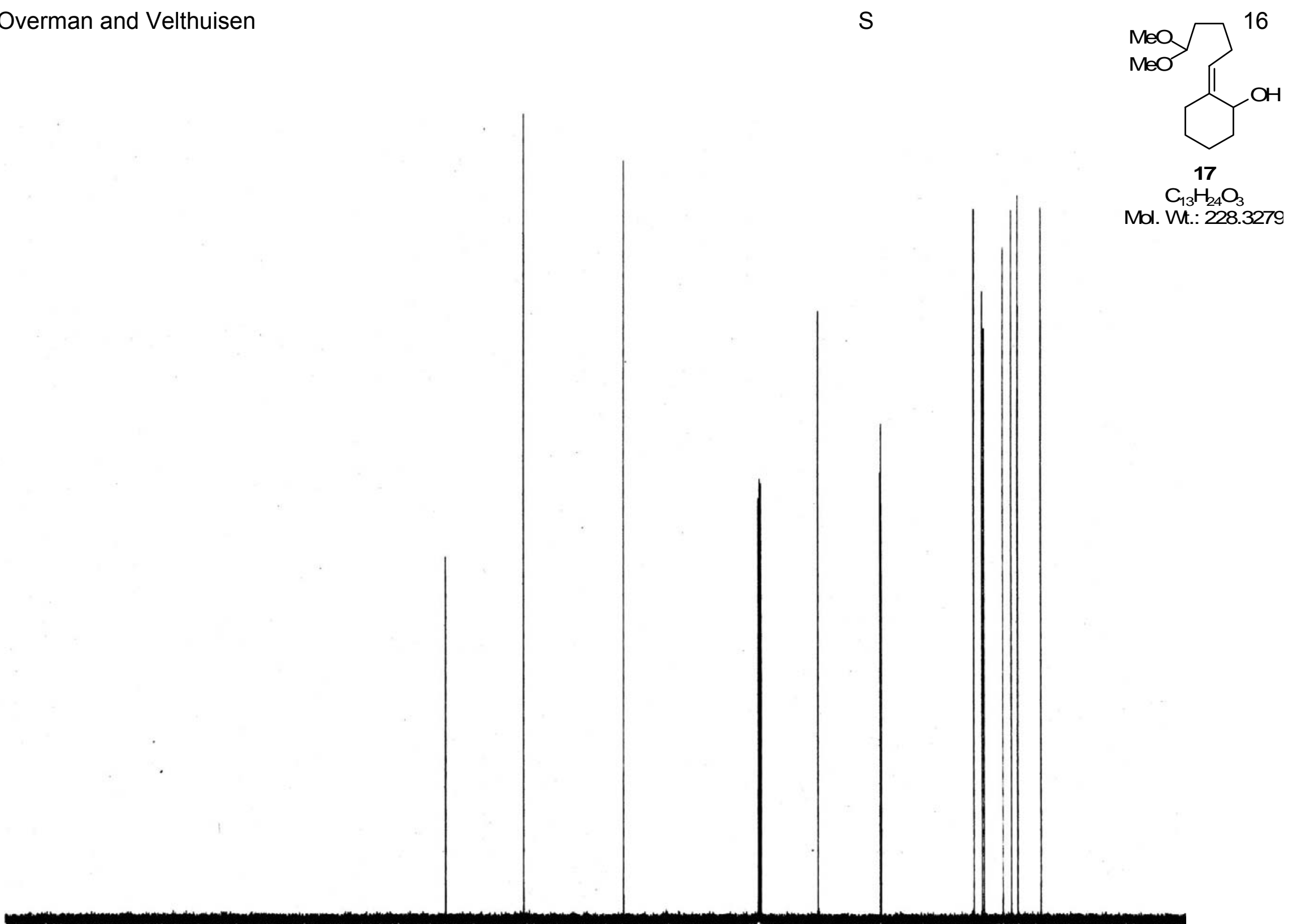

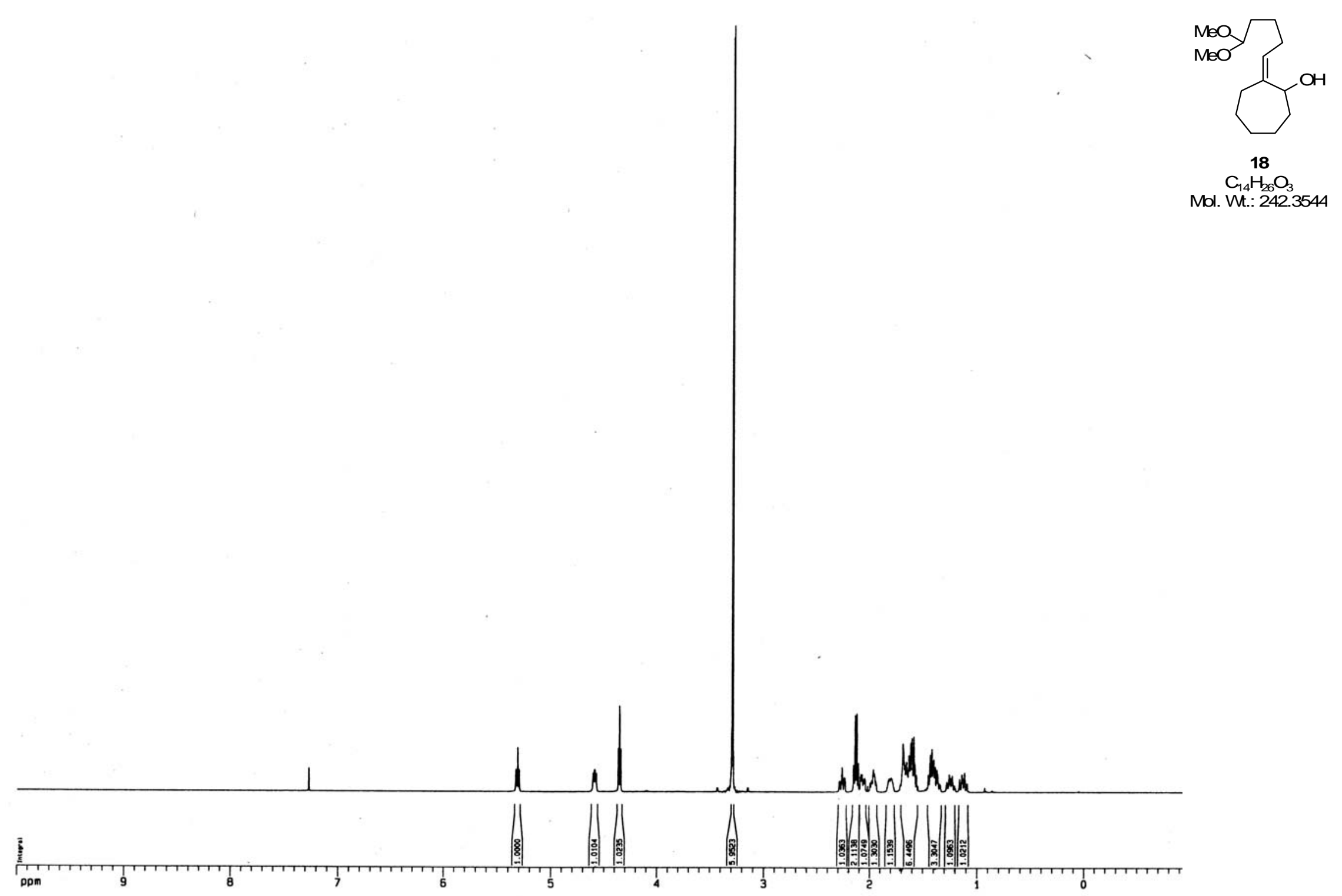


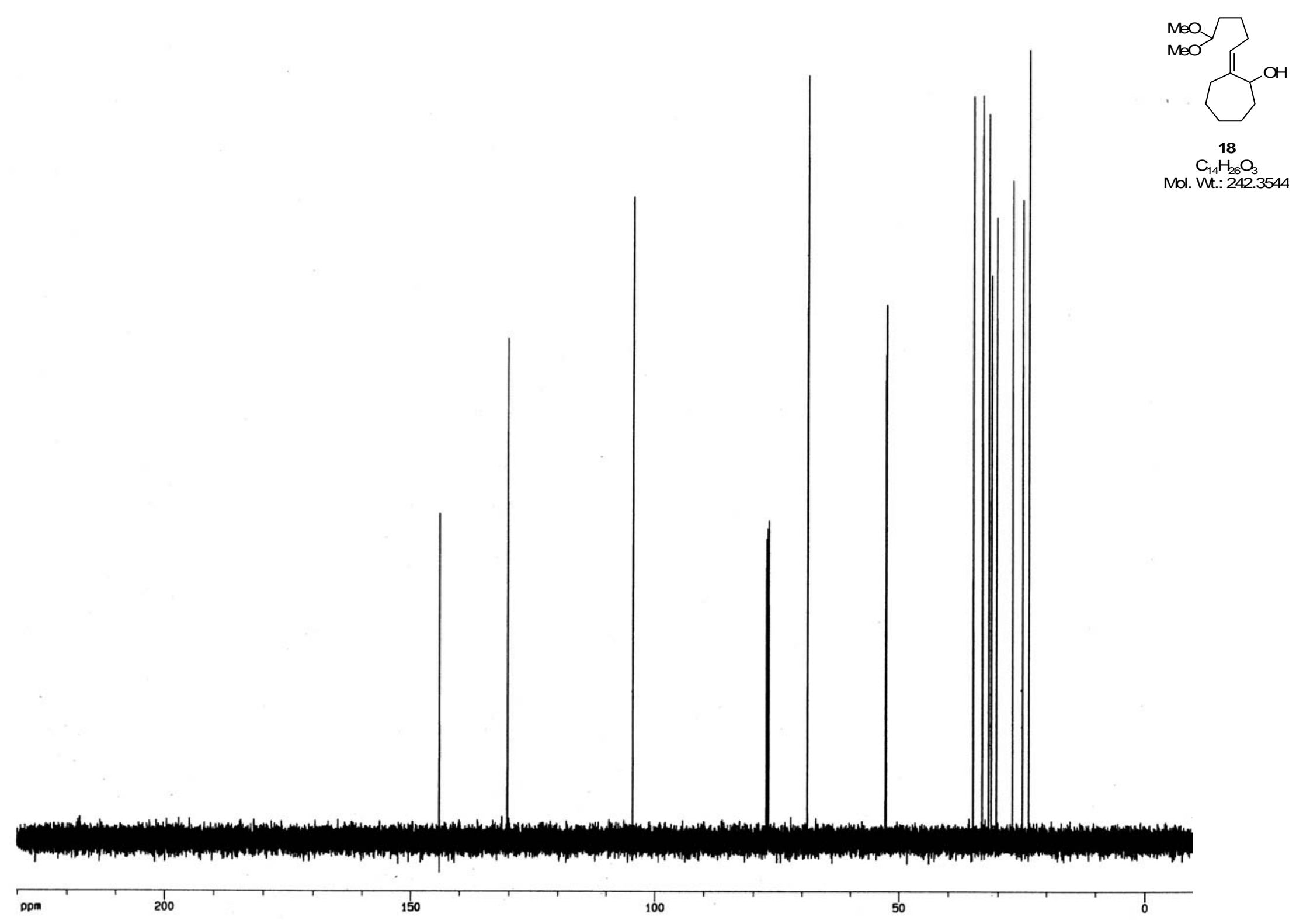




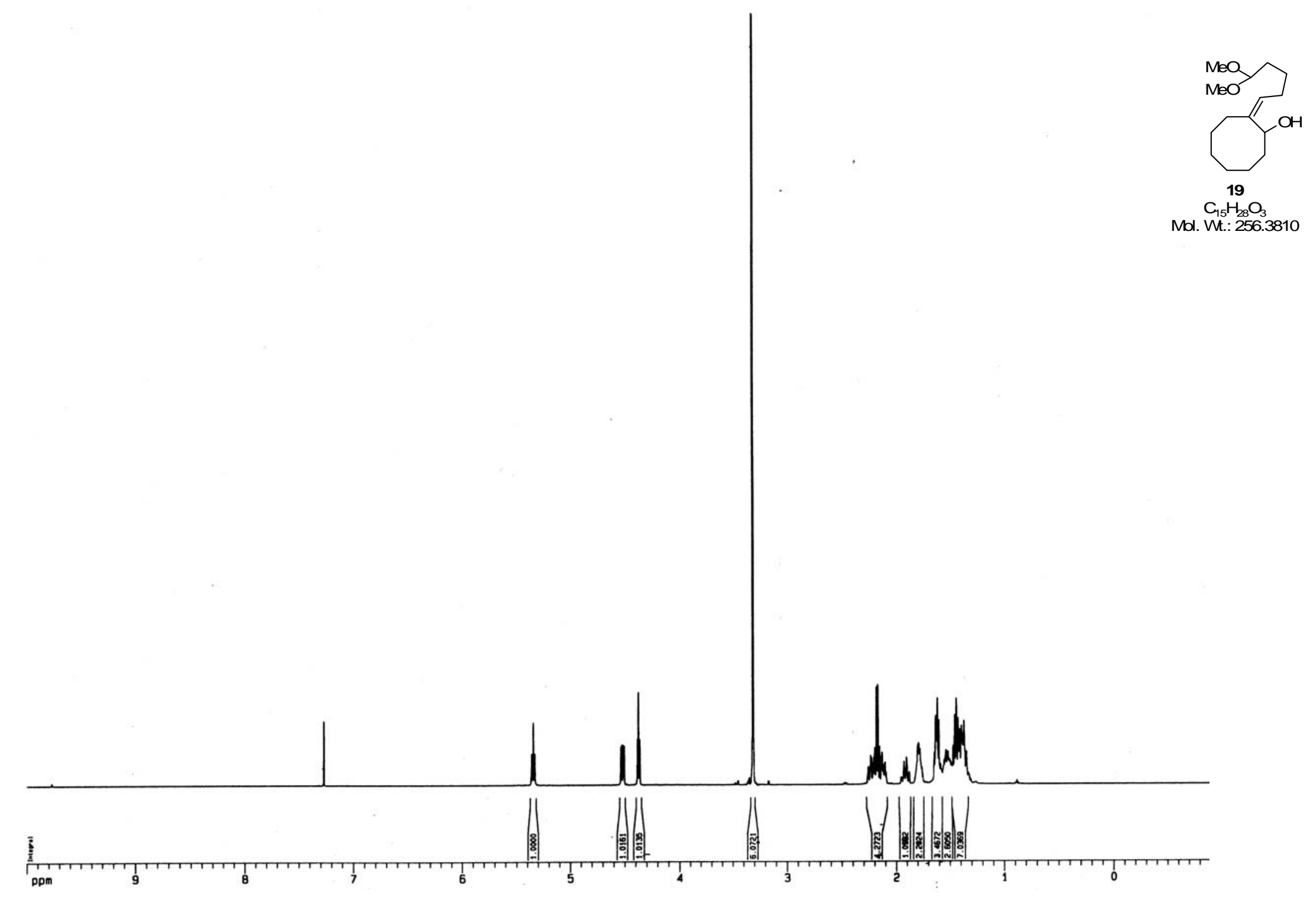




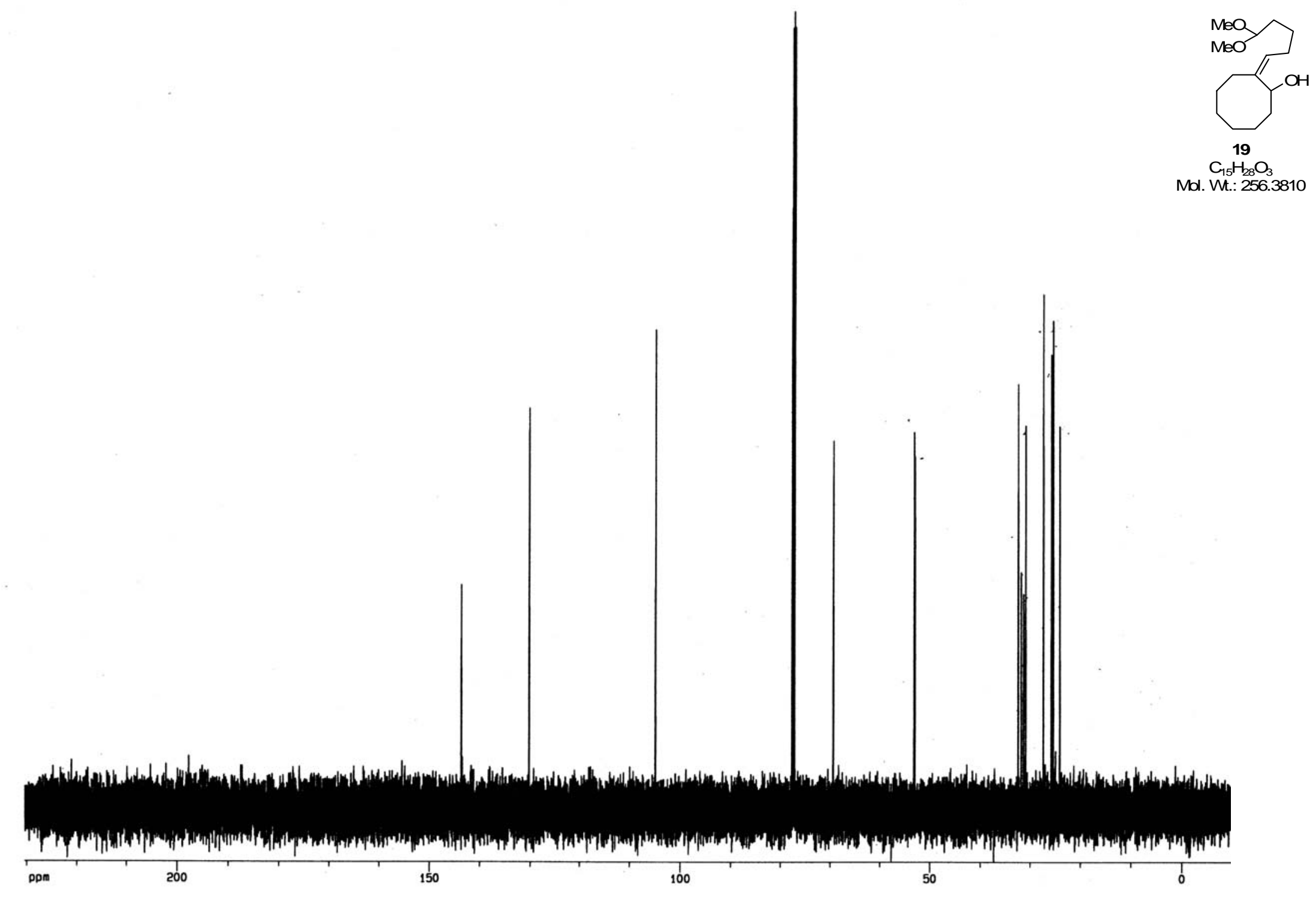




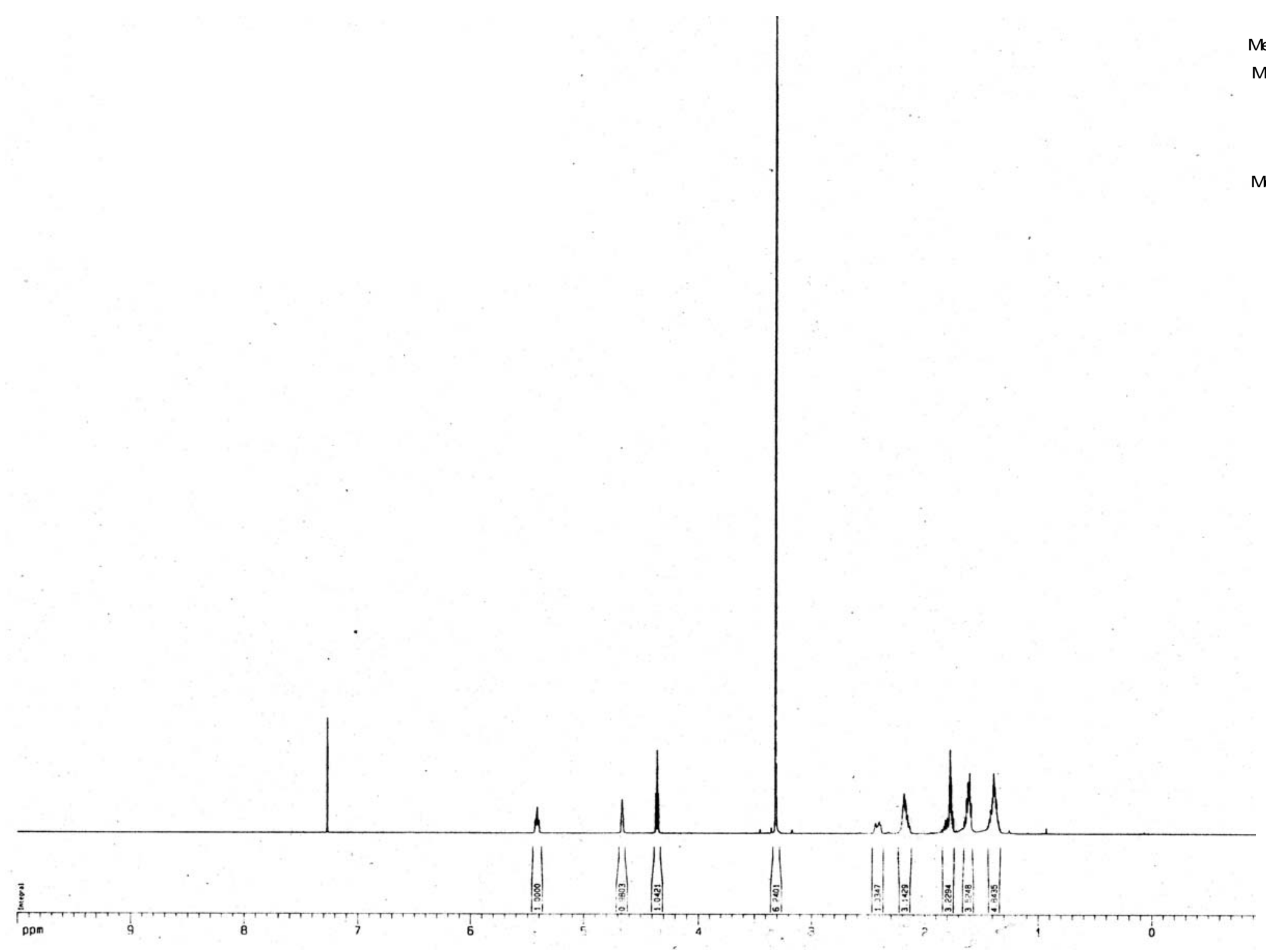

$\mathrm{MeO}$

MeO $\gamma^{\mathrm{OH}}$

28

$\mathrm{C}_{13} \mathrm{H}_{24} \mathrm{O}_{3}$ Mol. Wt.: 228.33 


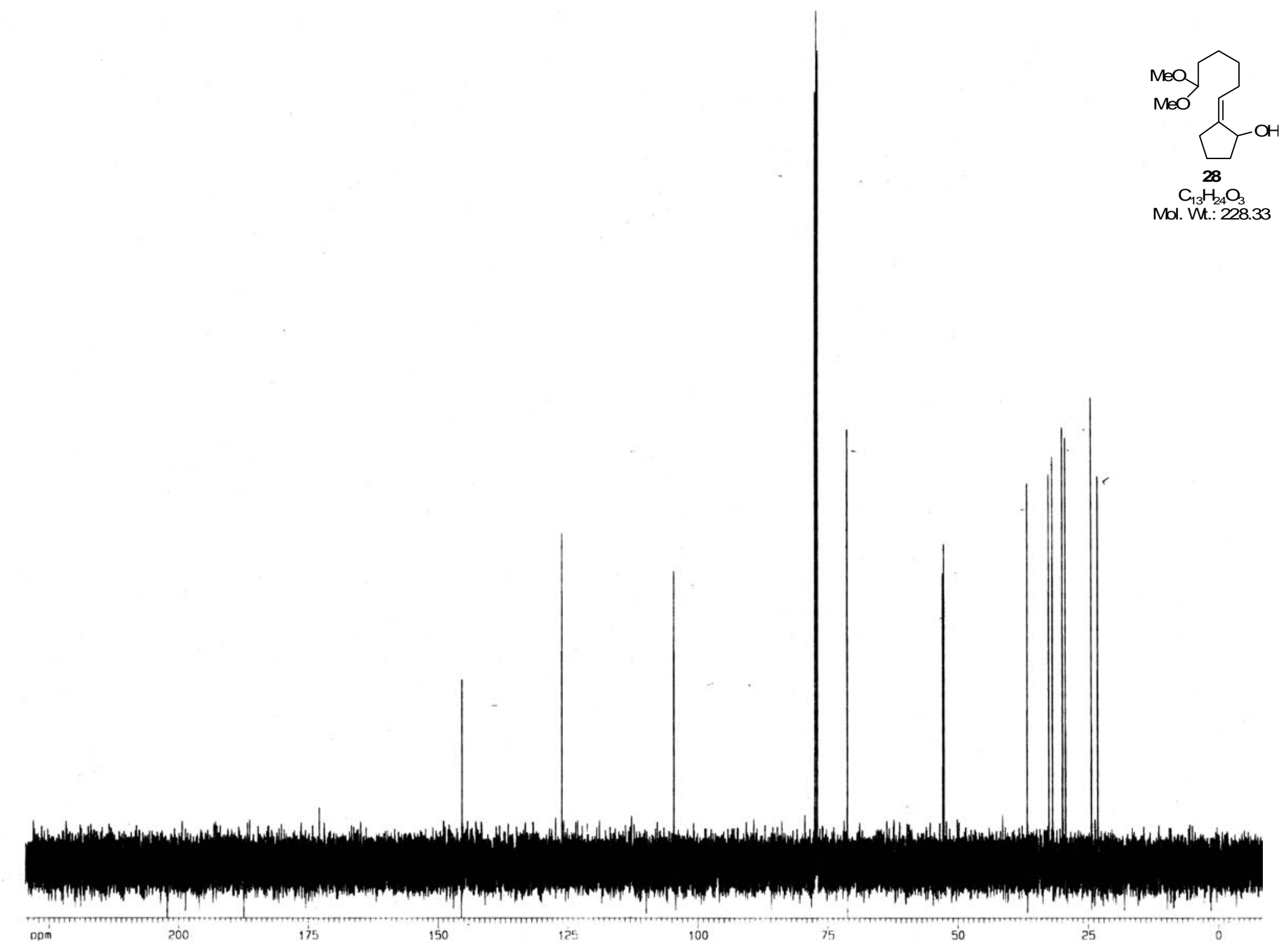




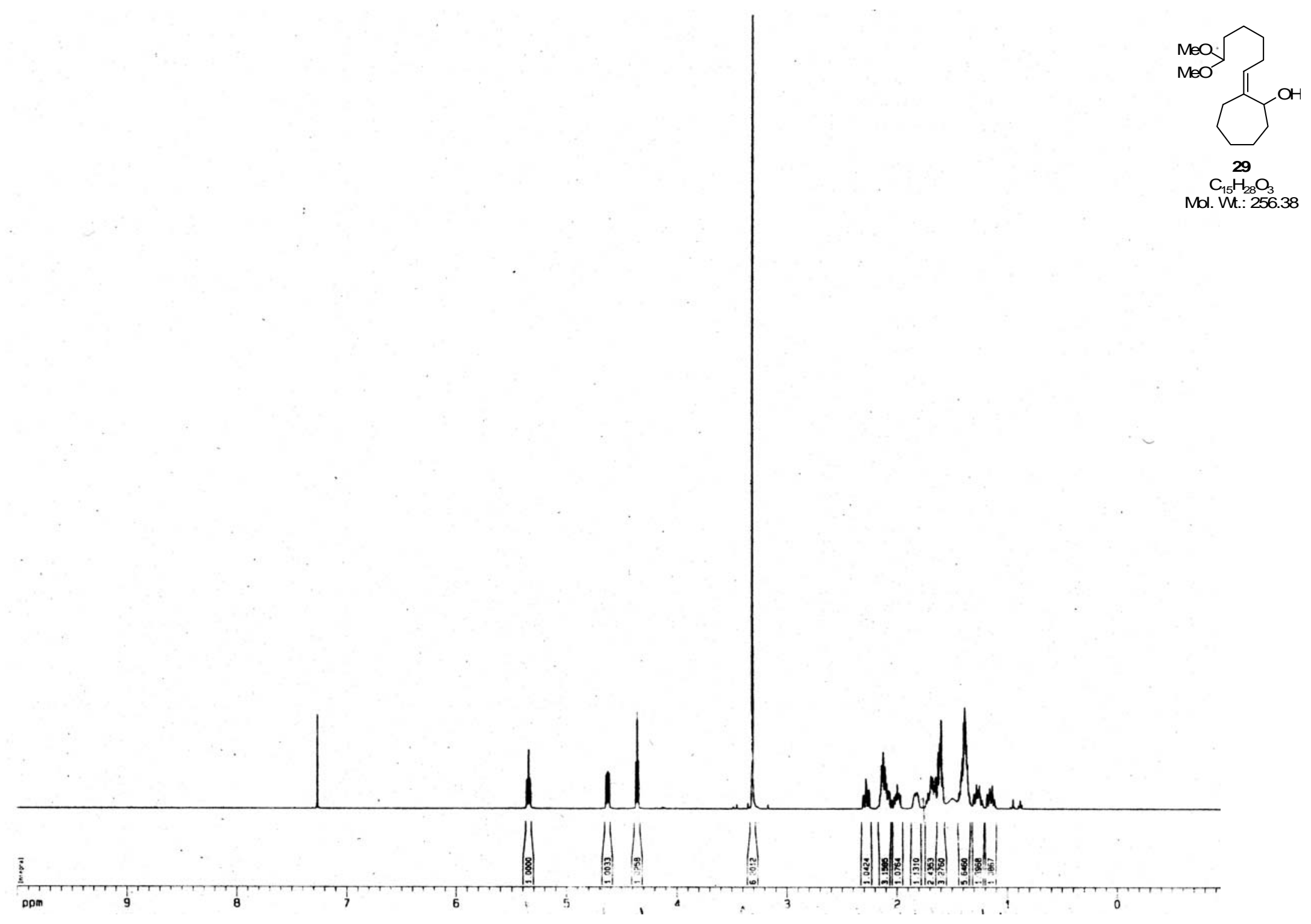




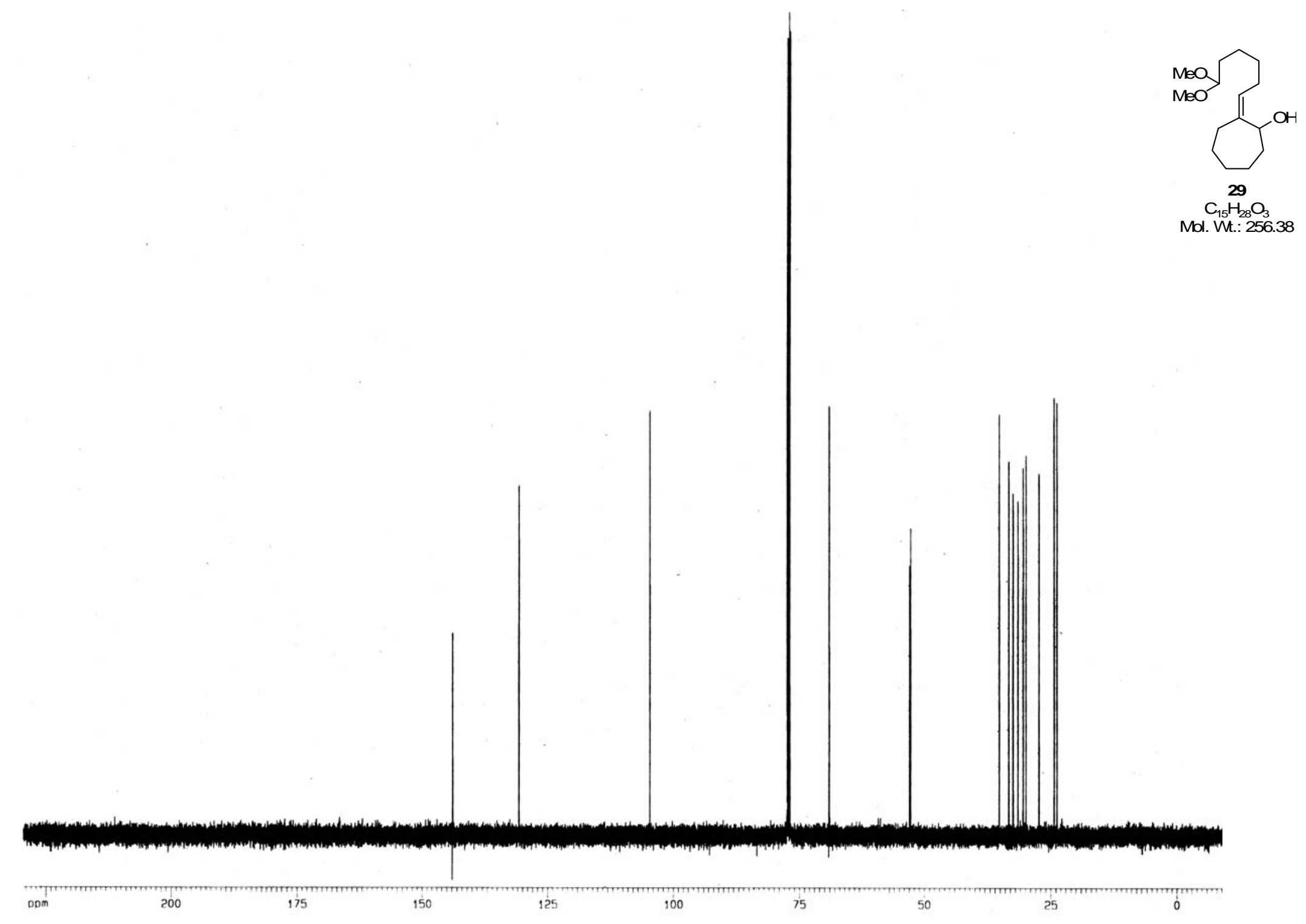




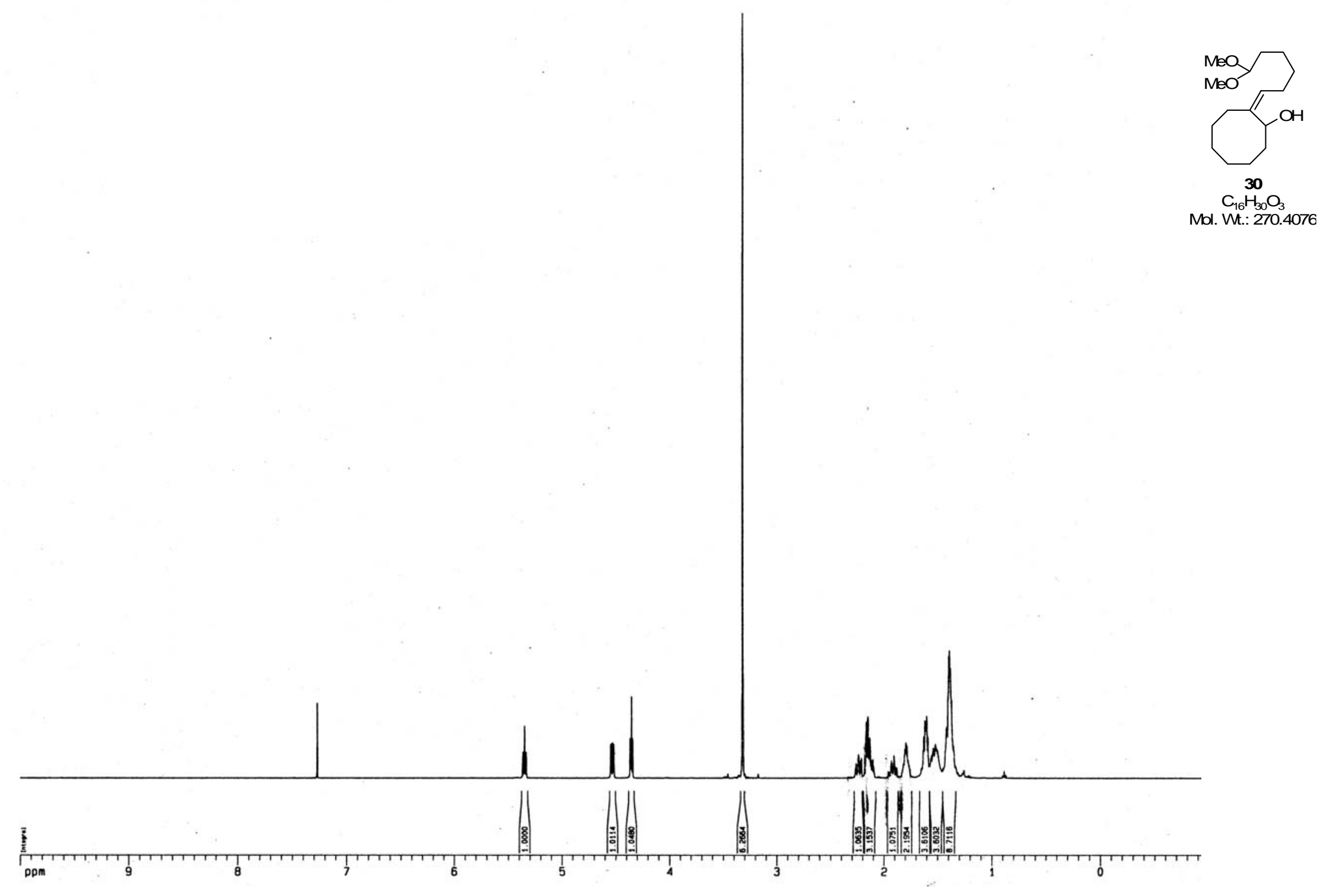




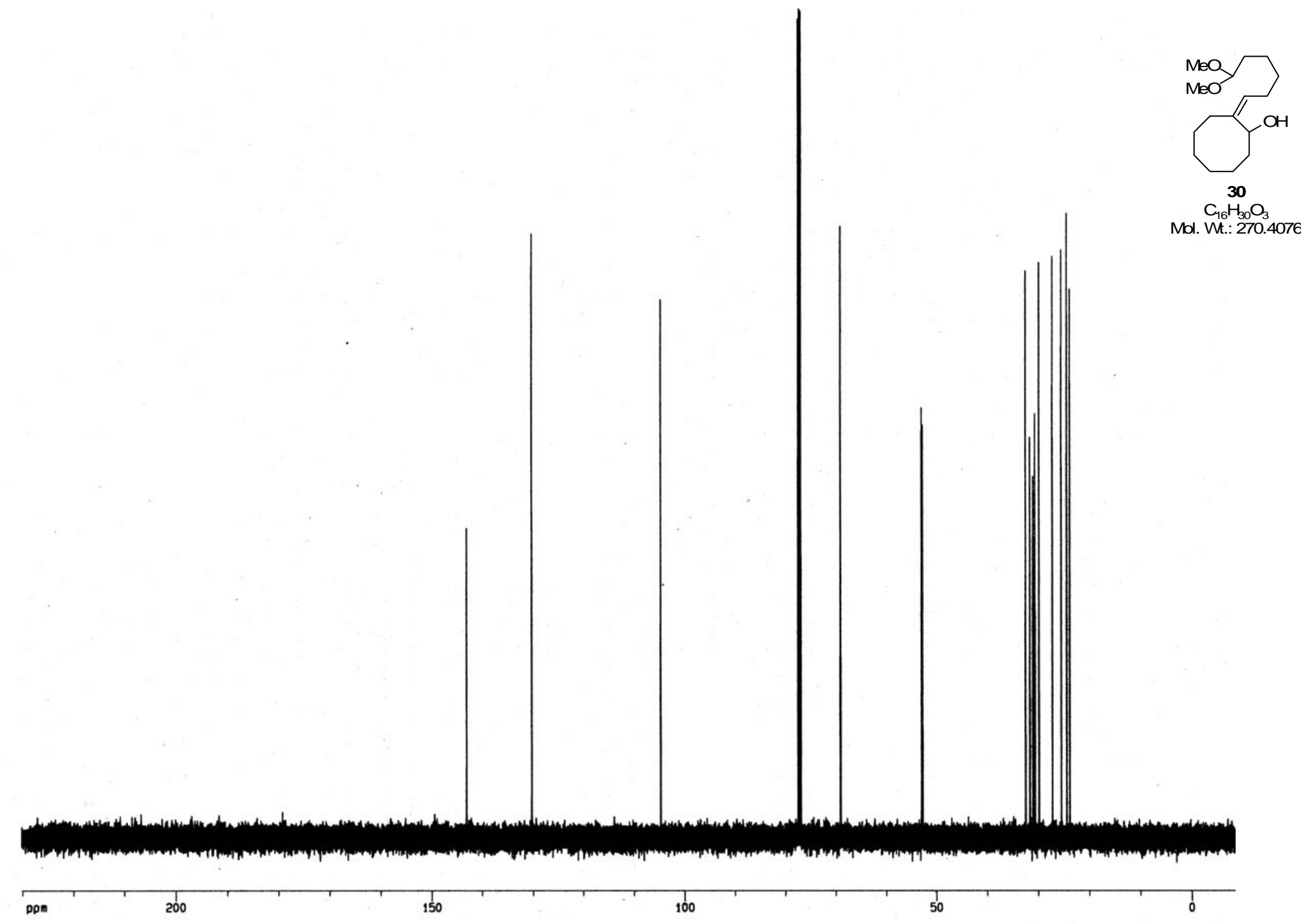




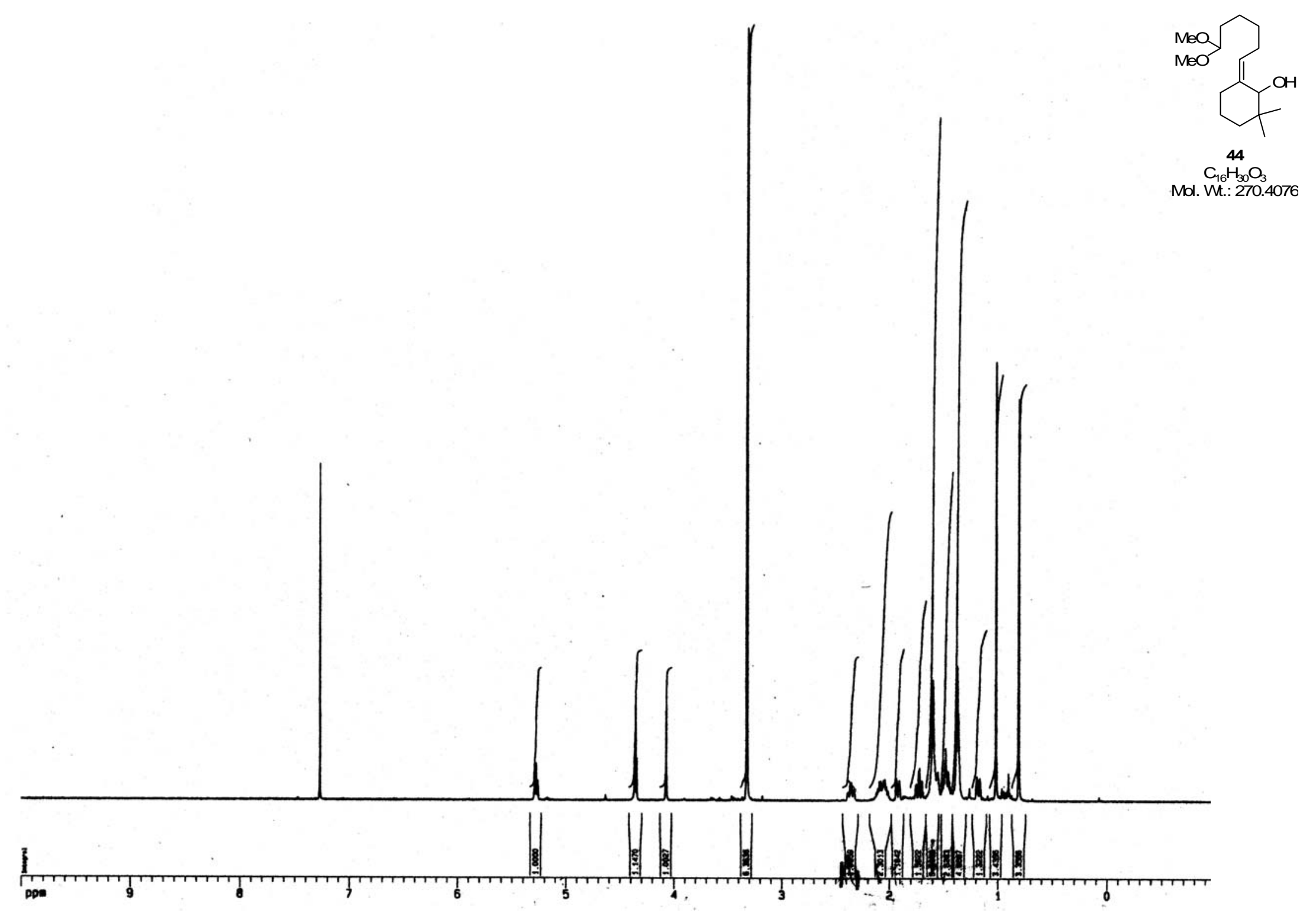




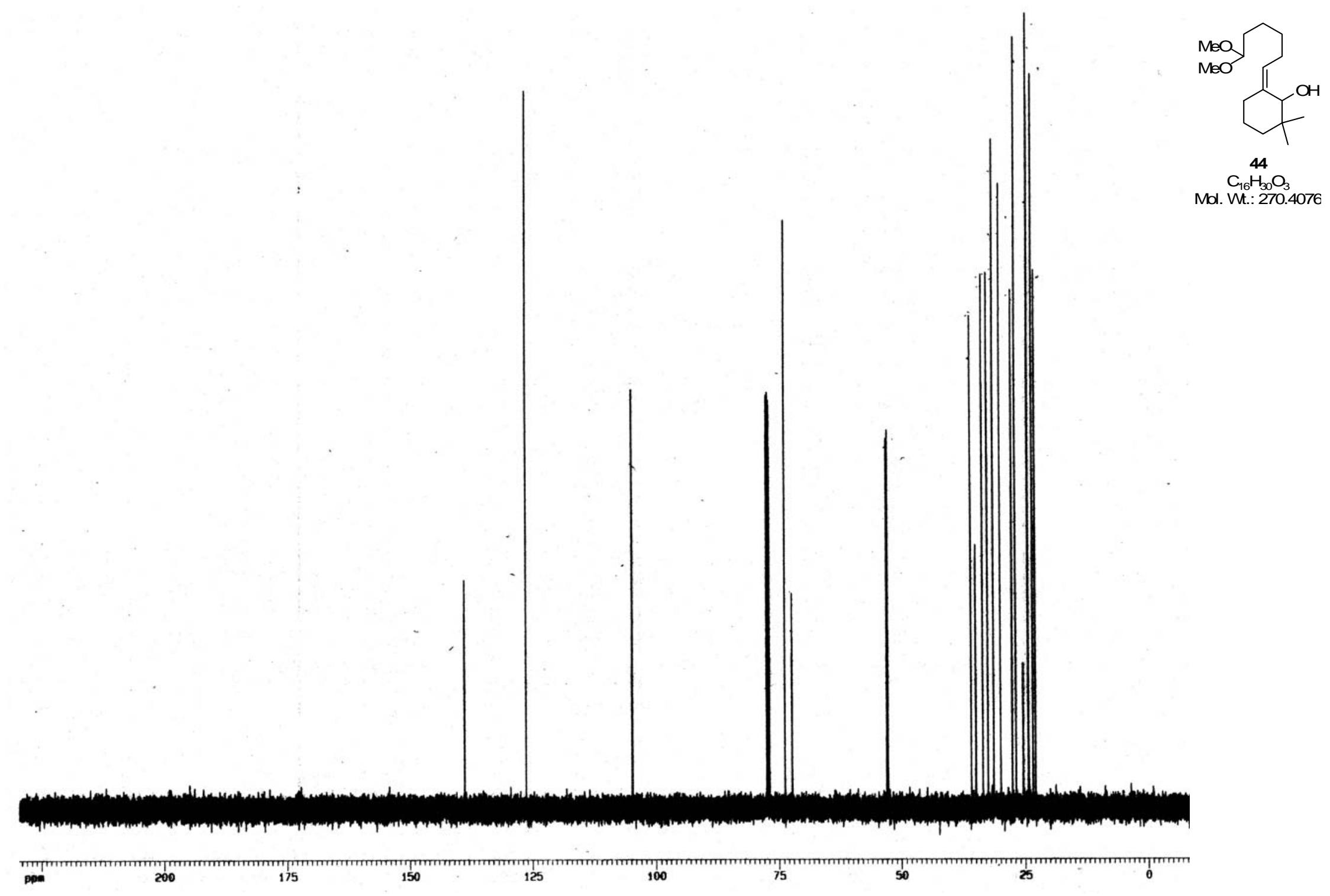




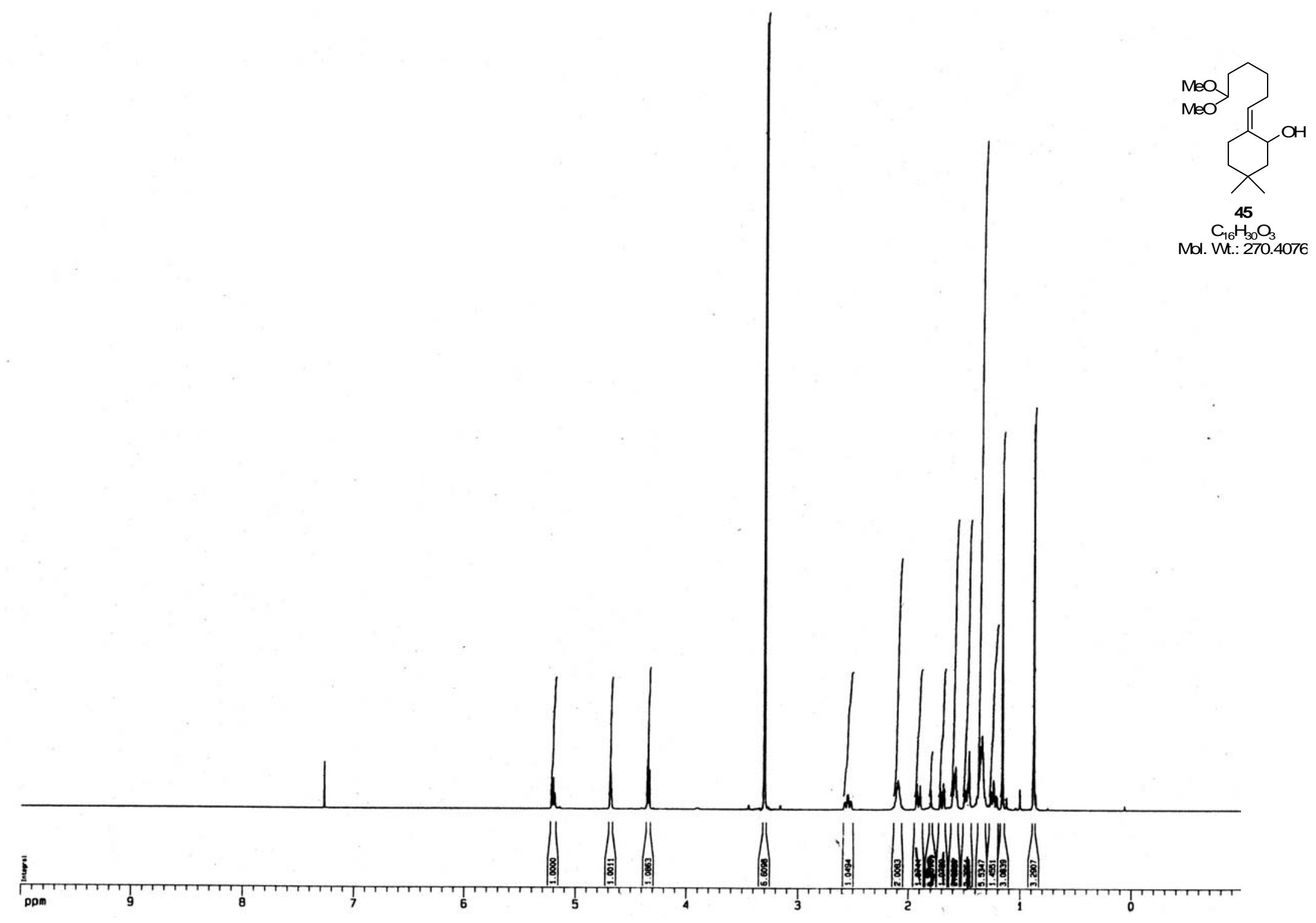




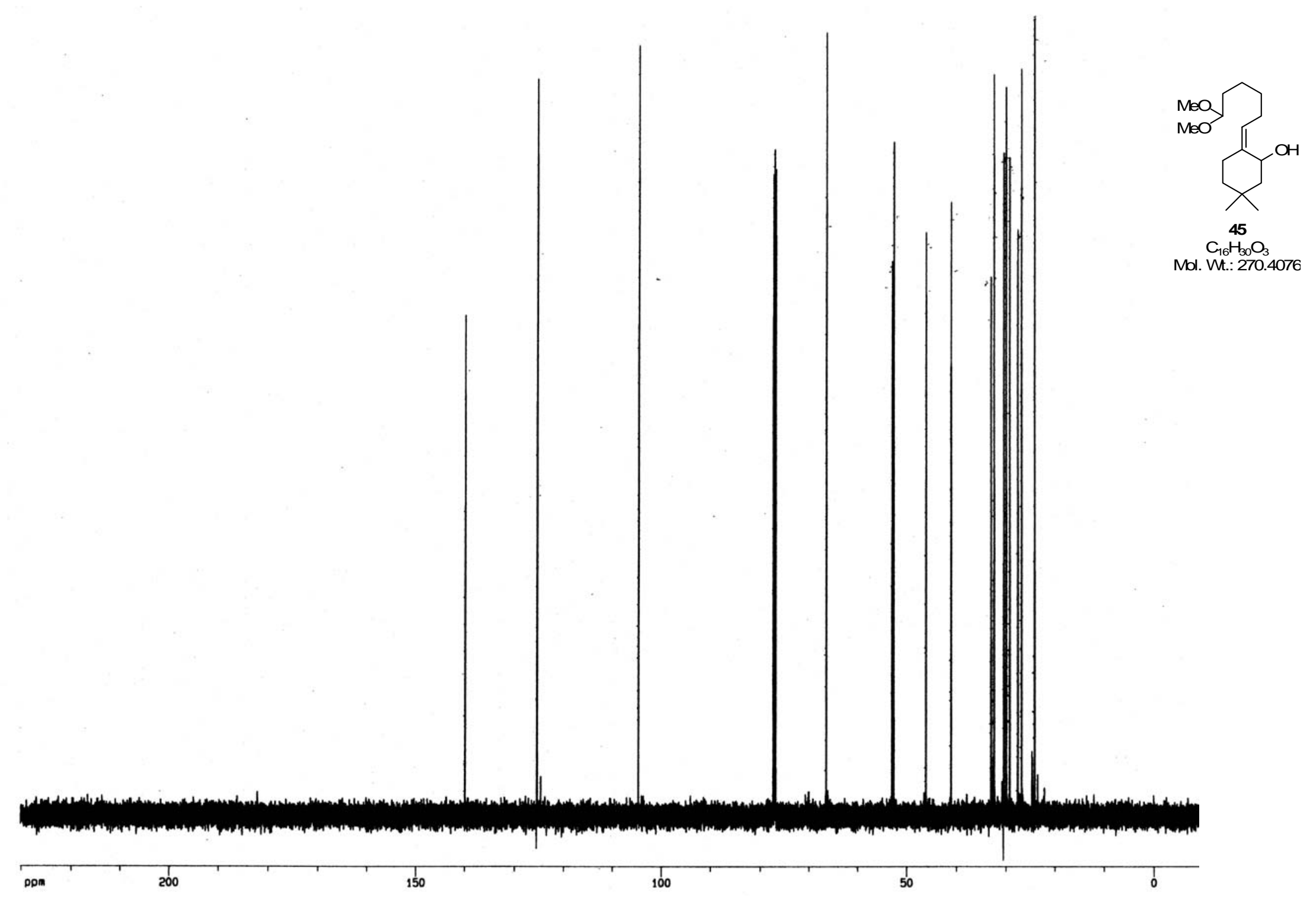




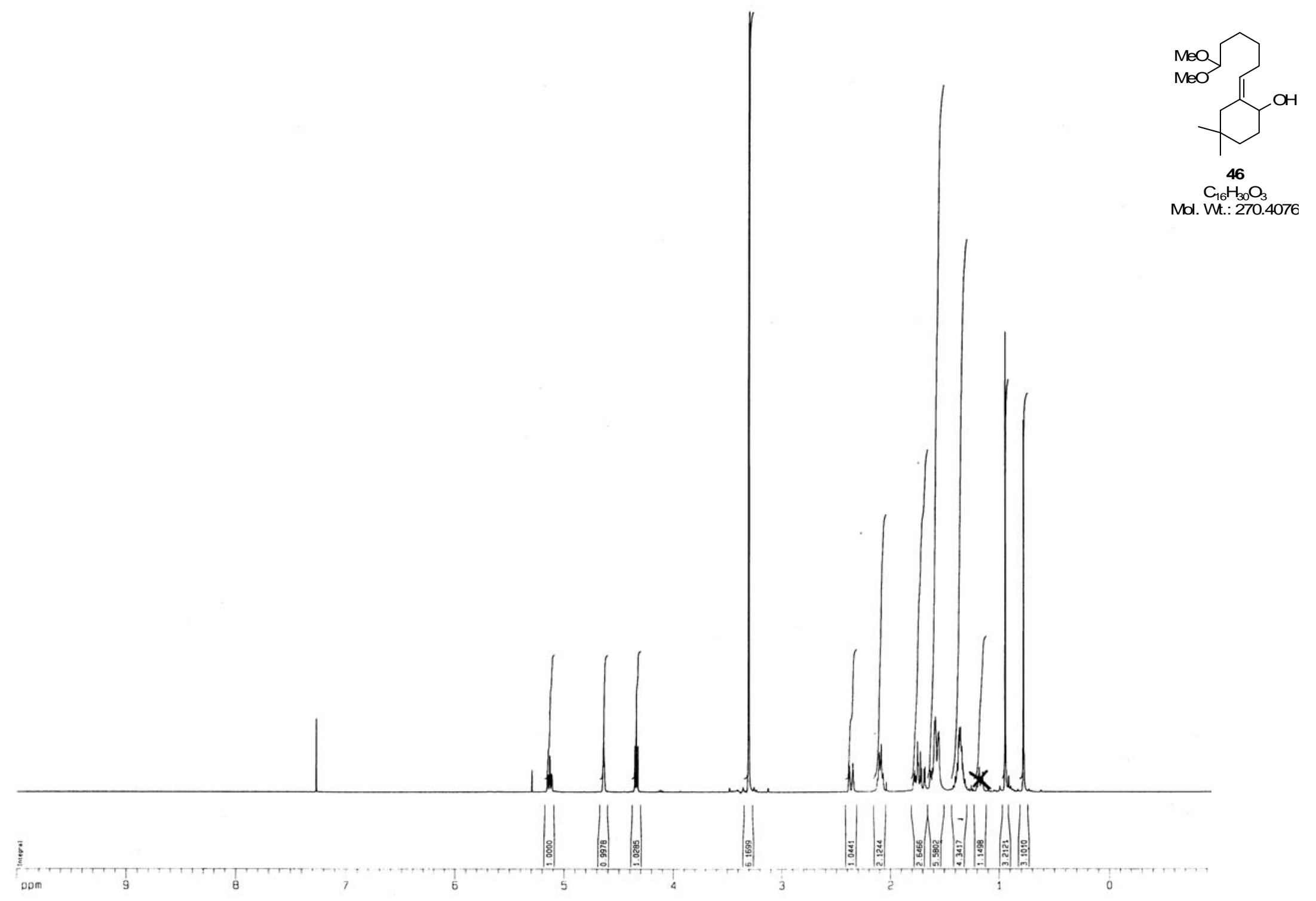




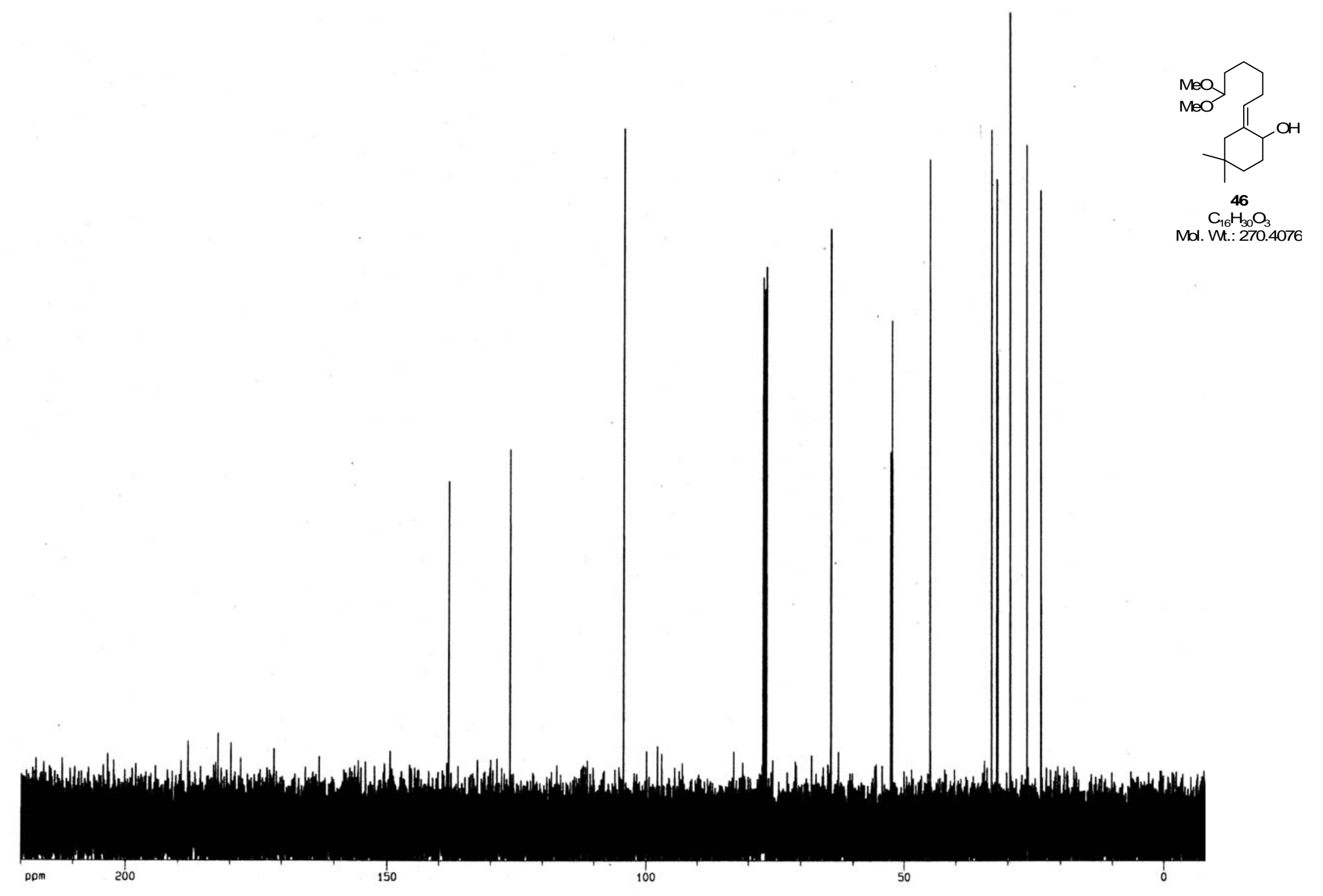




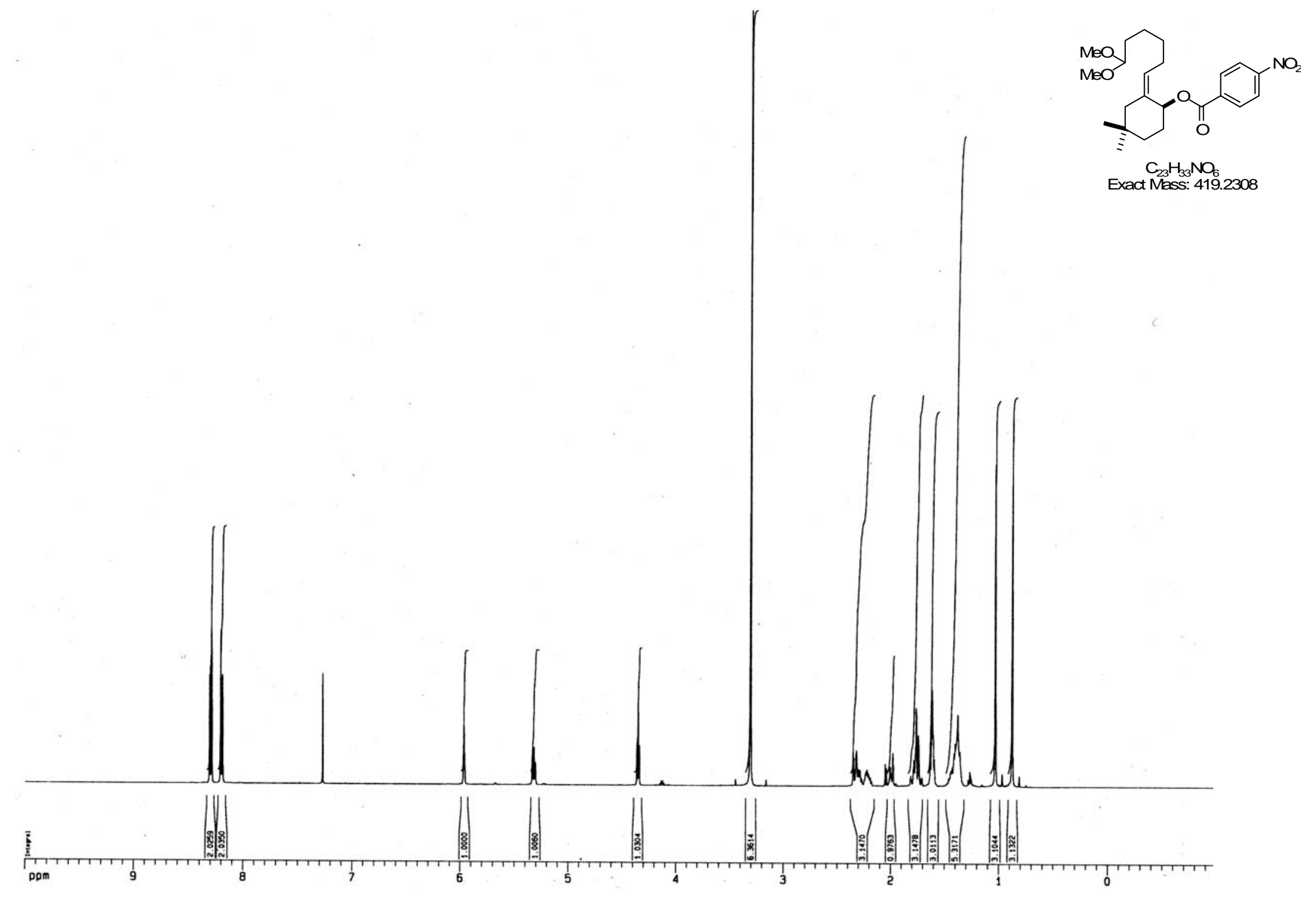




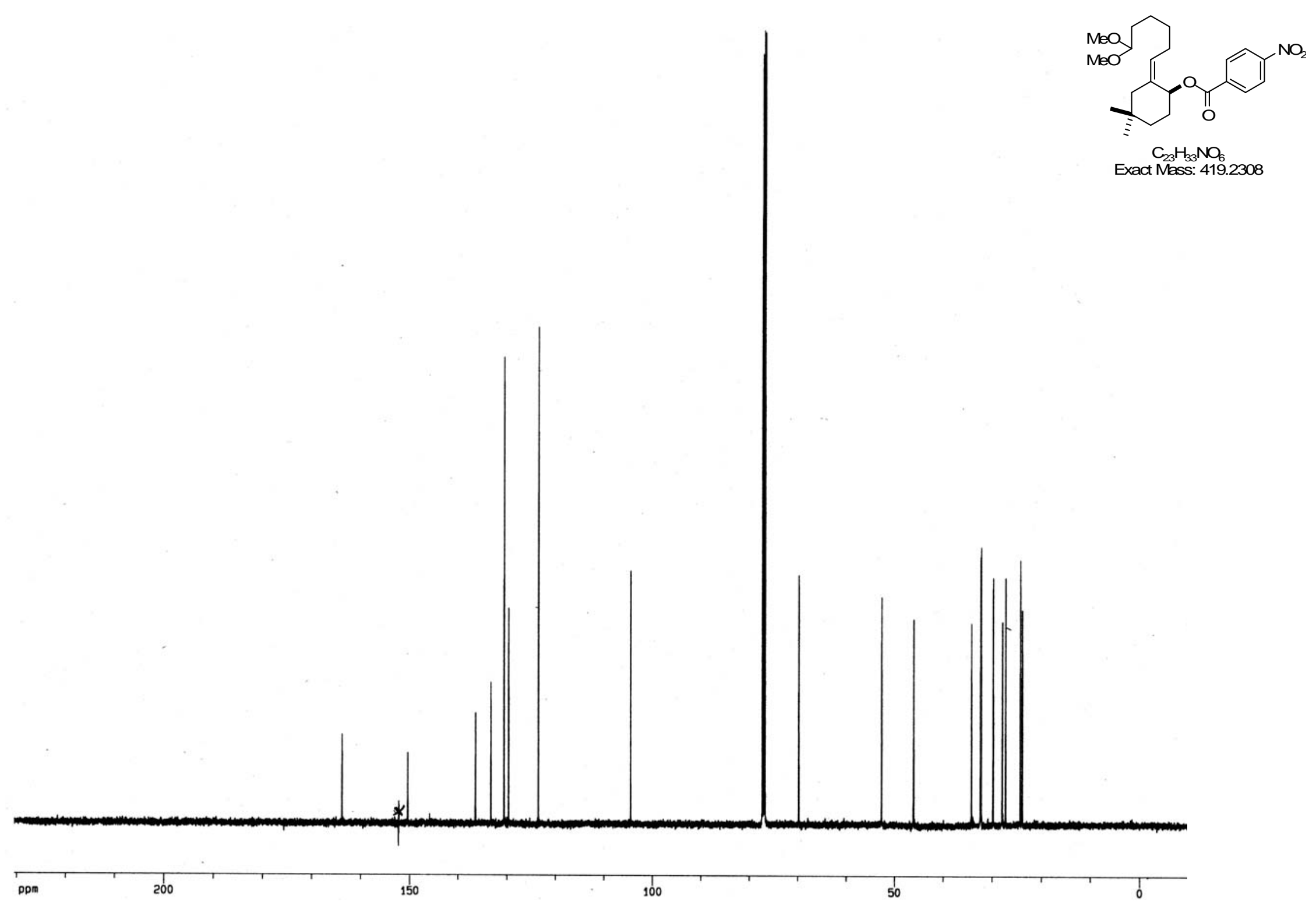


Data File C: \HPCHEM\IDDAA $99 / 2$ hexanes/IPA isocratic

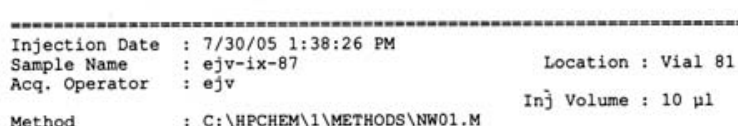

$\begin{array}{ll}\text { Method } & : \text { C: } \backslash \text { HPCHEM } 1 \text { 1 METHODS INW01.M } \\ \text { Last changed } & : 7 / 30 / 05 \quad 1: 27: 21 \text { PM by ejv }\end{array}$

$2 \times$ obh, 58 iProH $/ 958$ Hexanes, $0.5 \mathrm{~mL} / \mathrm{min}$

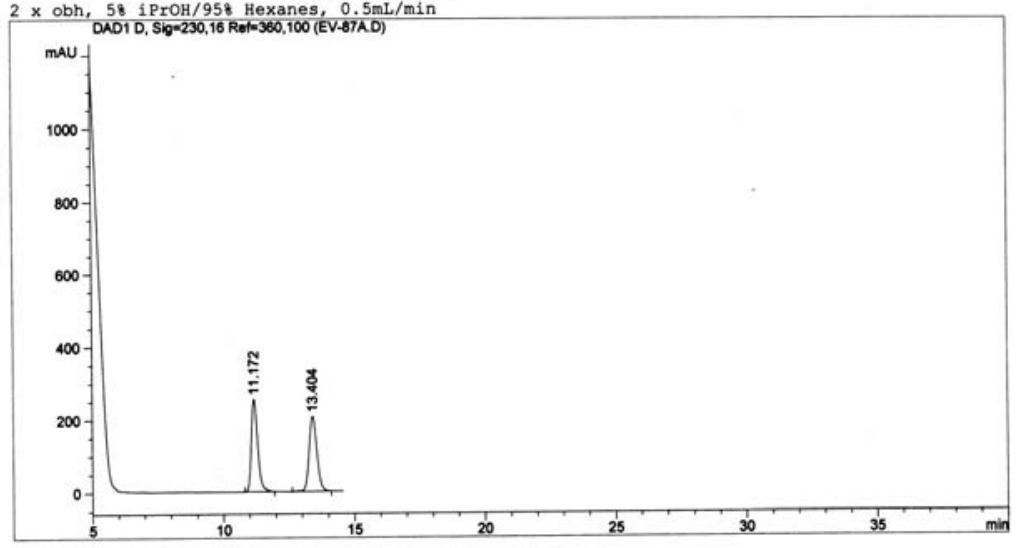

Area Percent Report

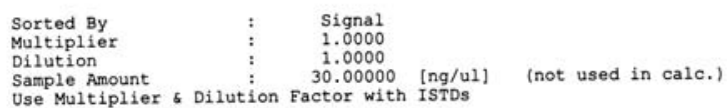

Sample Amount

Signal 1: DAD1 D, Sig=230,16 Ref-360,100

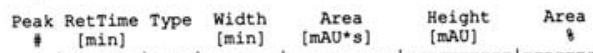

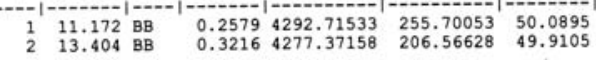

$\begin{array}{lll}\text { Totals : } & 8570.08691 \quad 462.26682\end{array}$

Results obtained with enhanced integrator!

... End of Report *...
Sample Name: ejv-ix-87

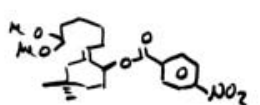

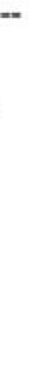

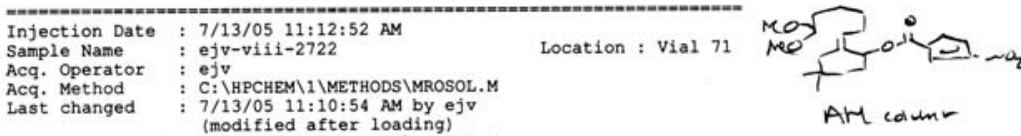

(modified after loading)

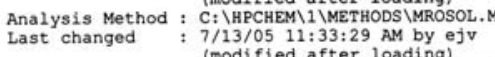
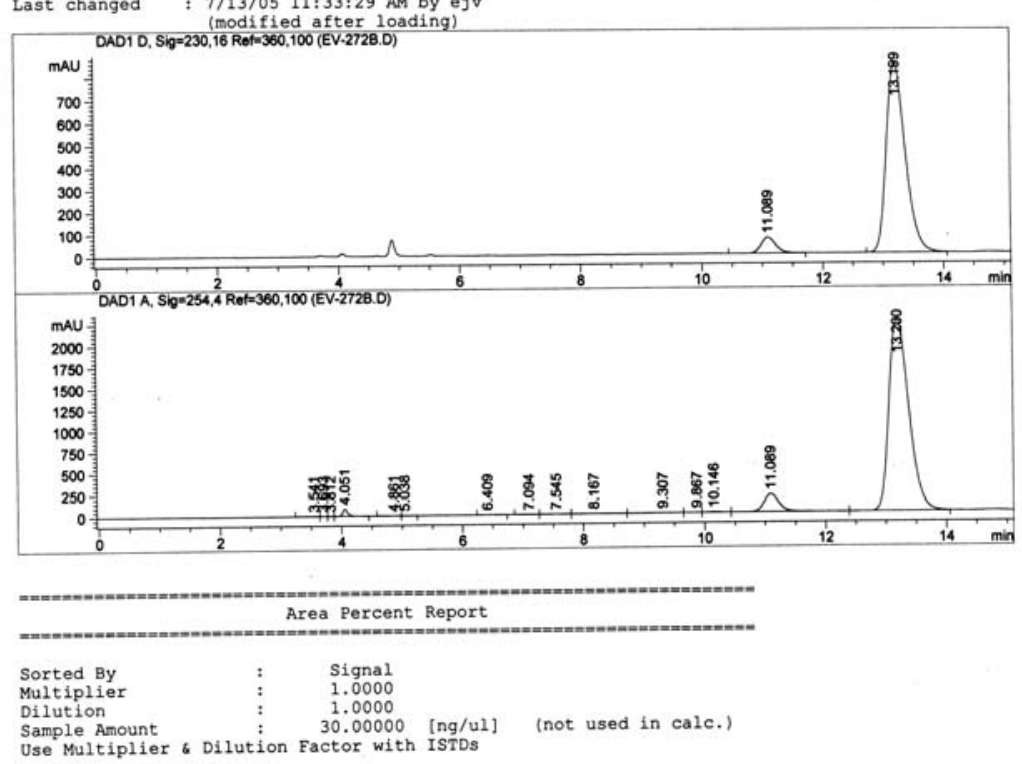

Signal 1: DAD1 D, Sig-230,16 Ref $=360,100$

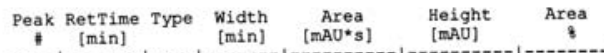

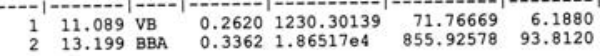
Tota1s: $\quad 1.9882004 \quad 927.69247$

Results obtained with enhanced integrator! 


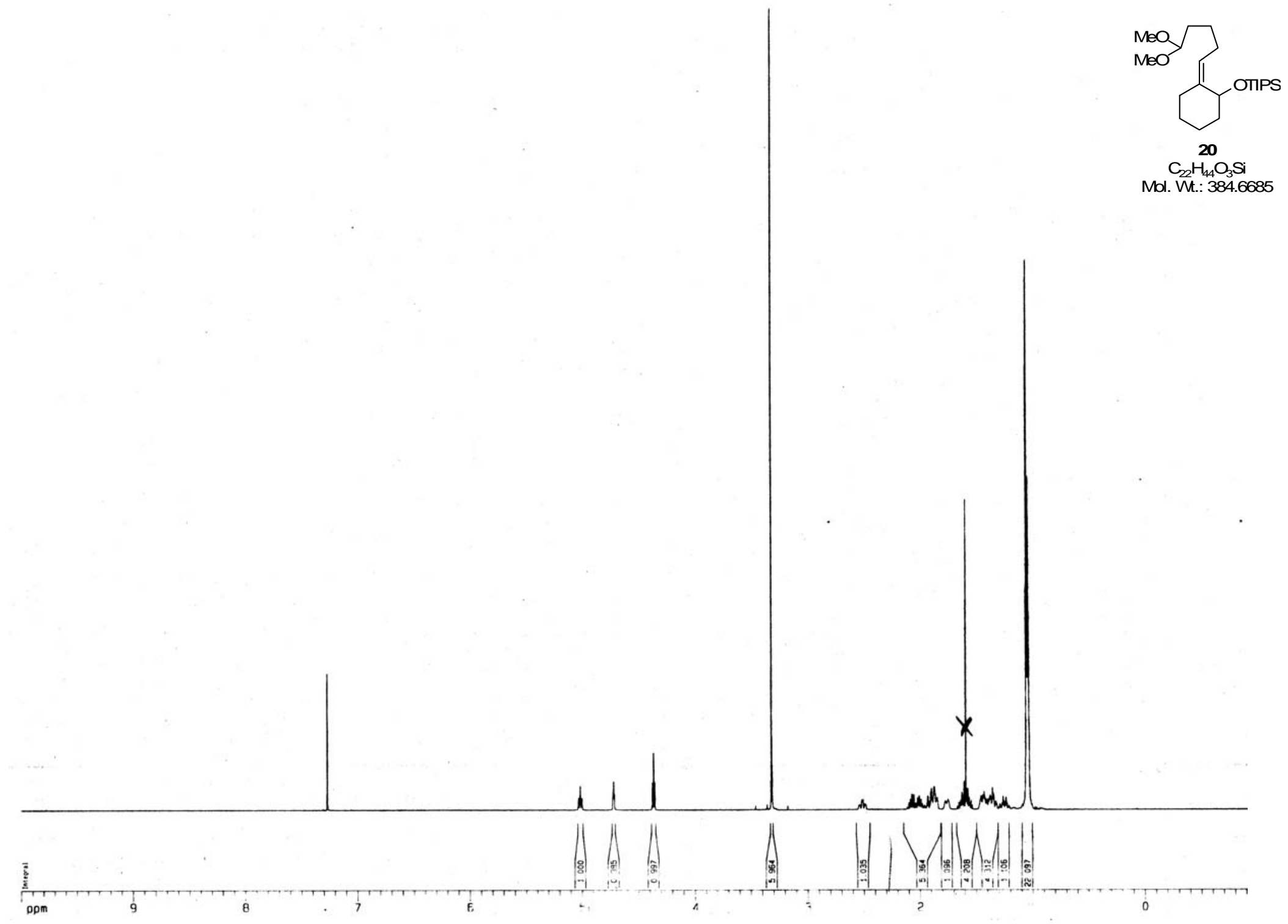




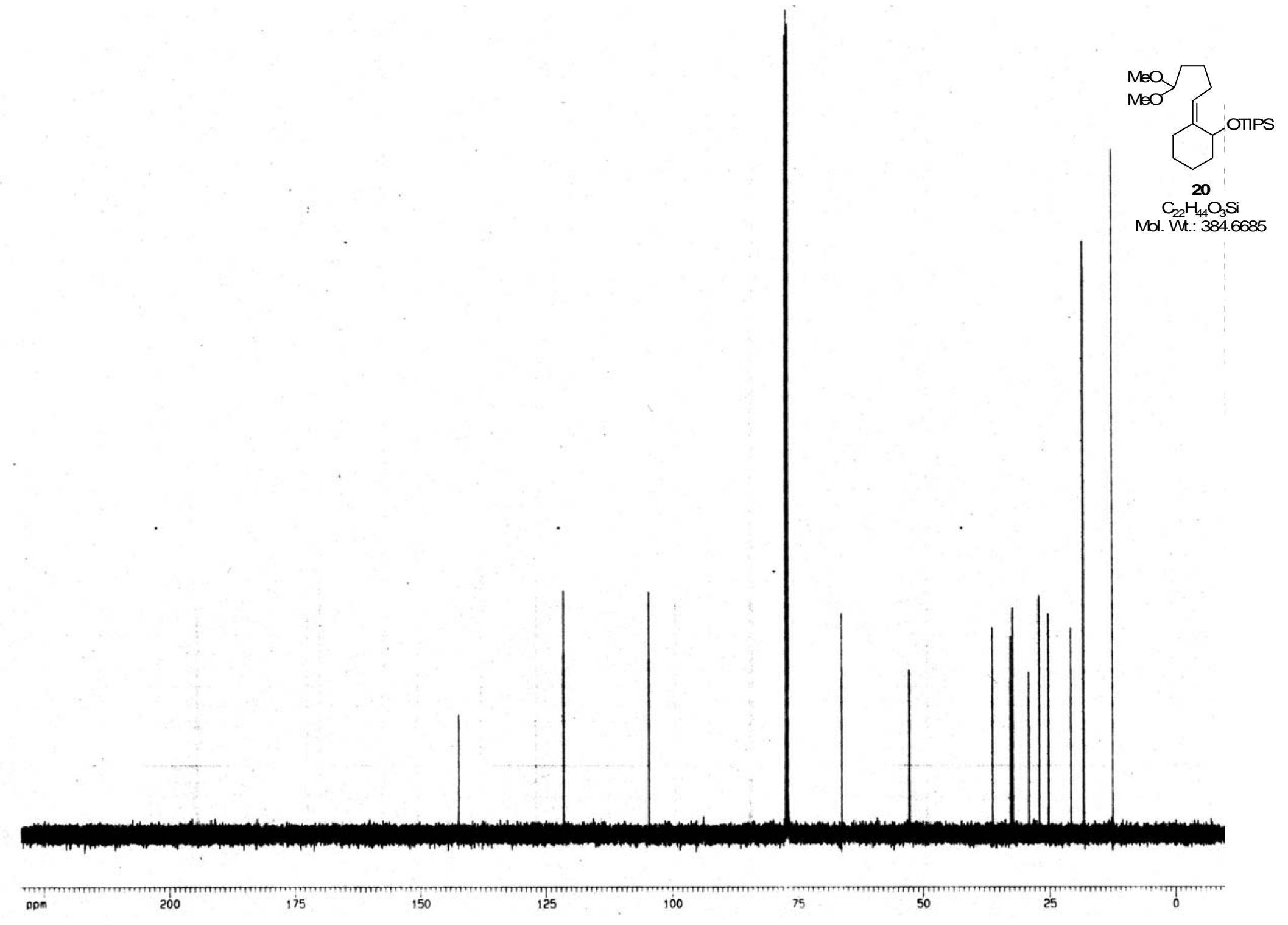




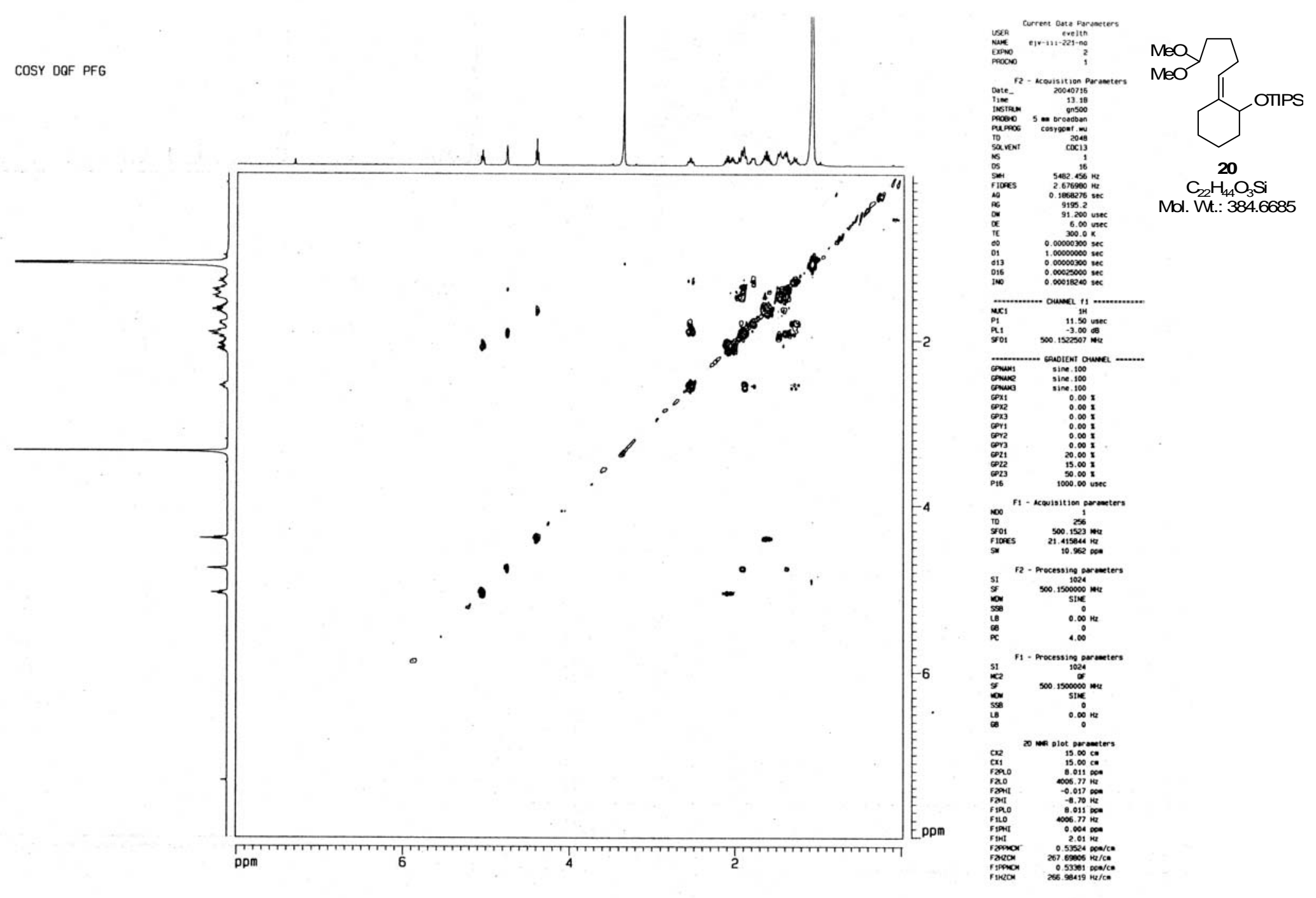



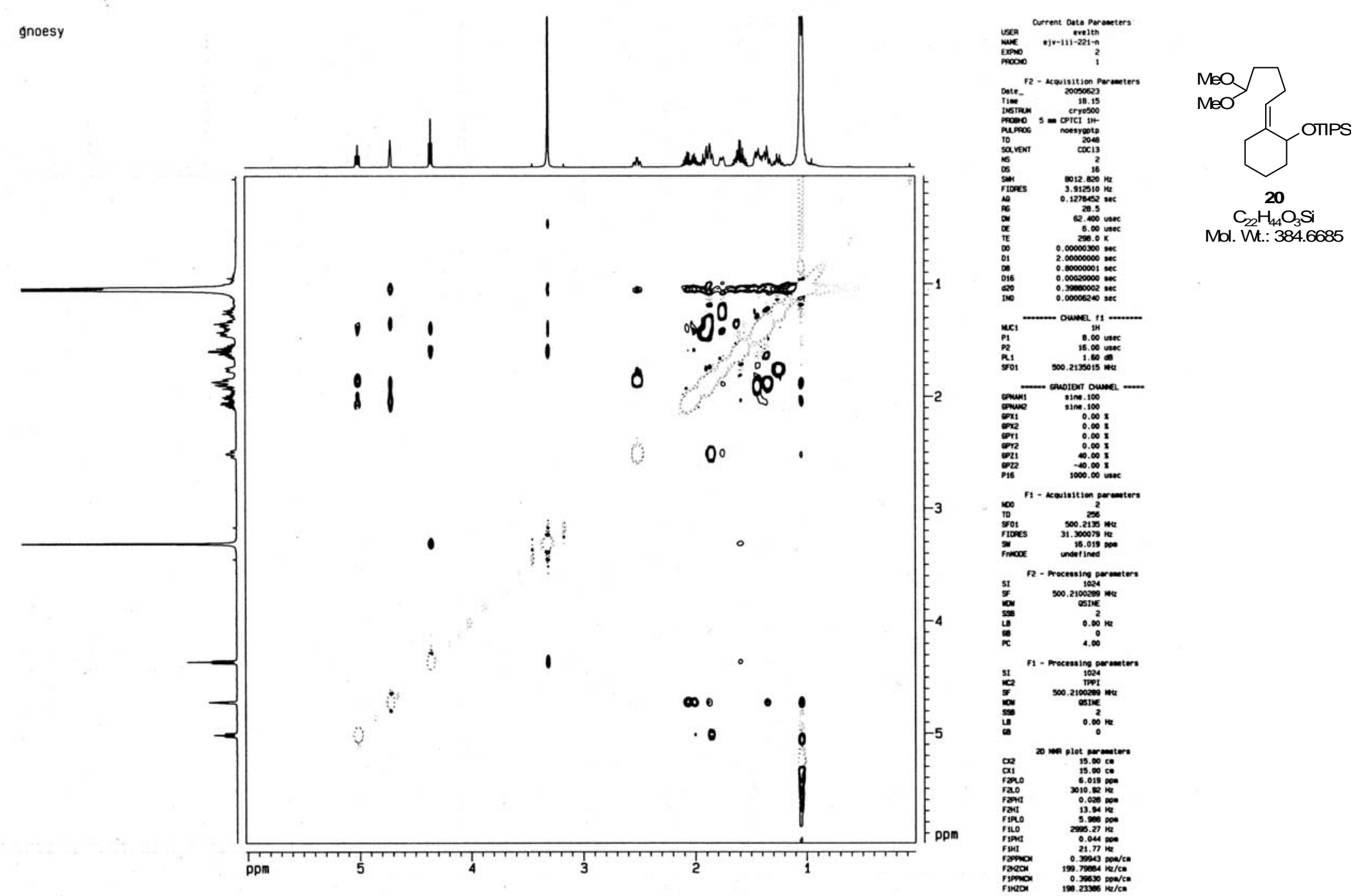


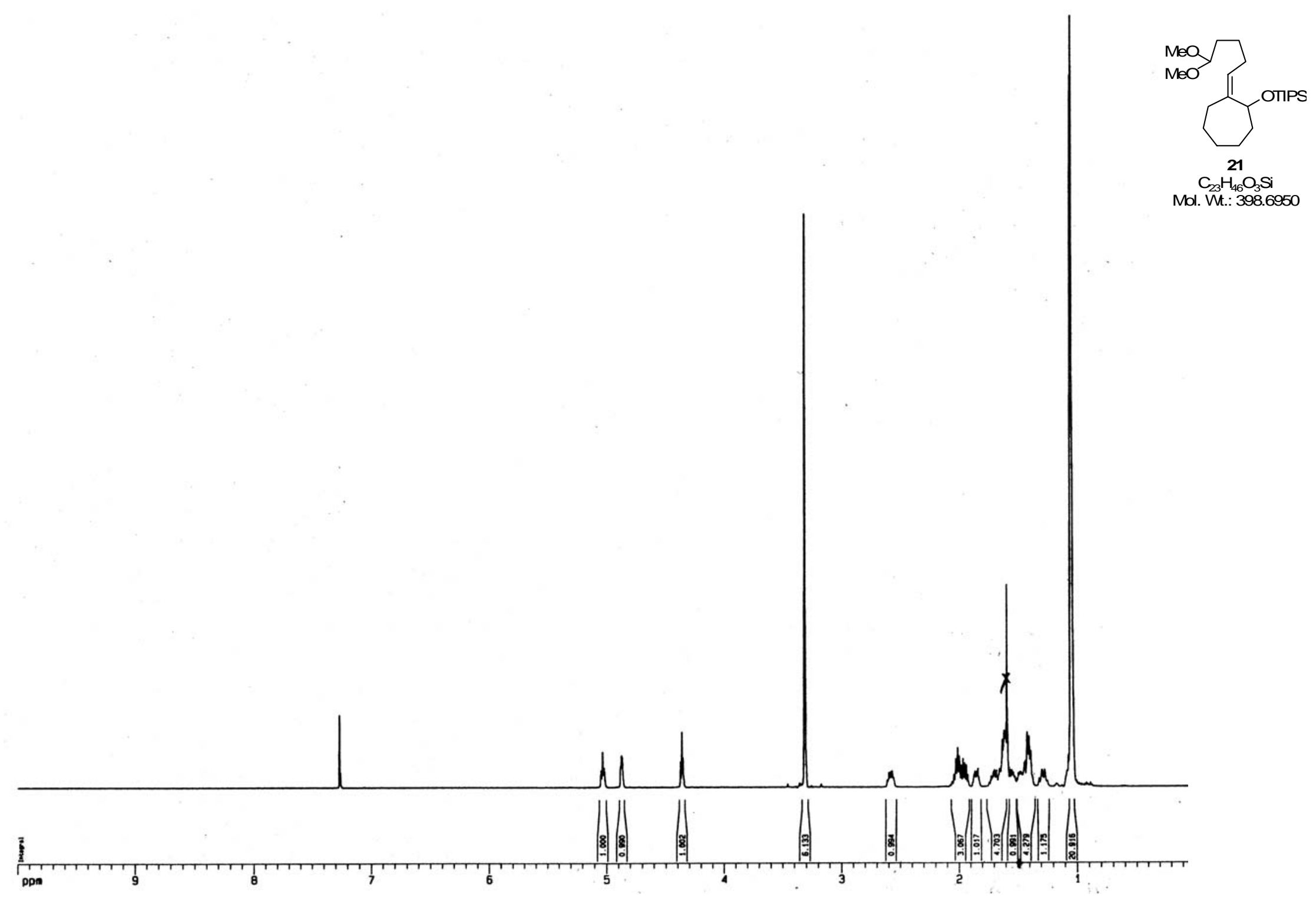




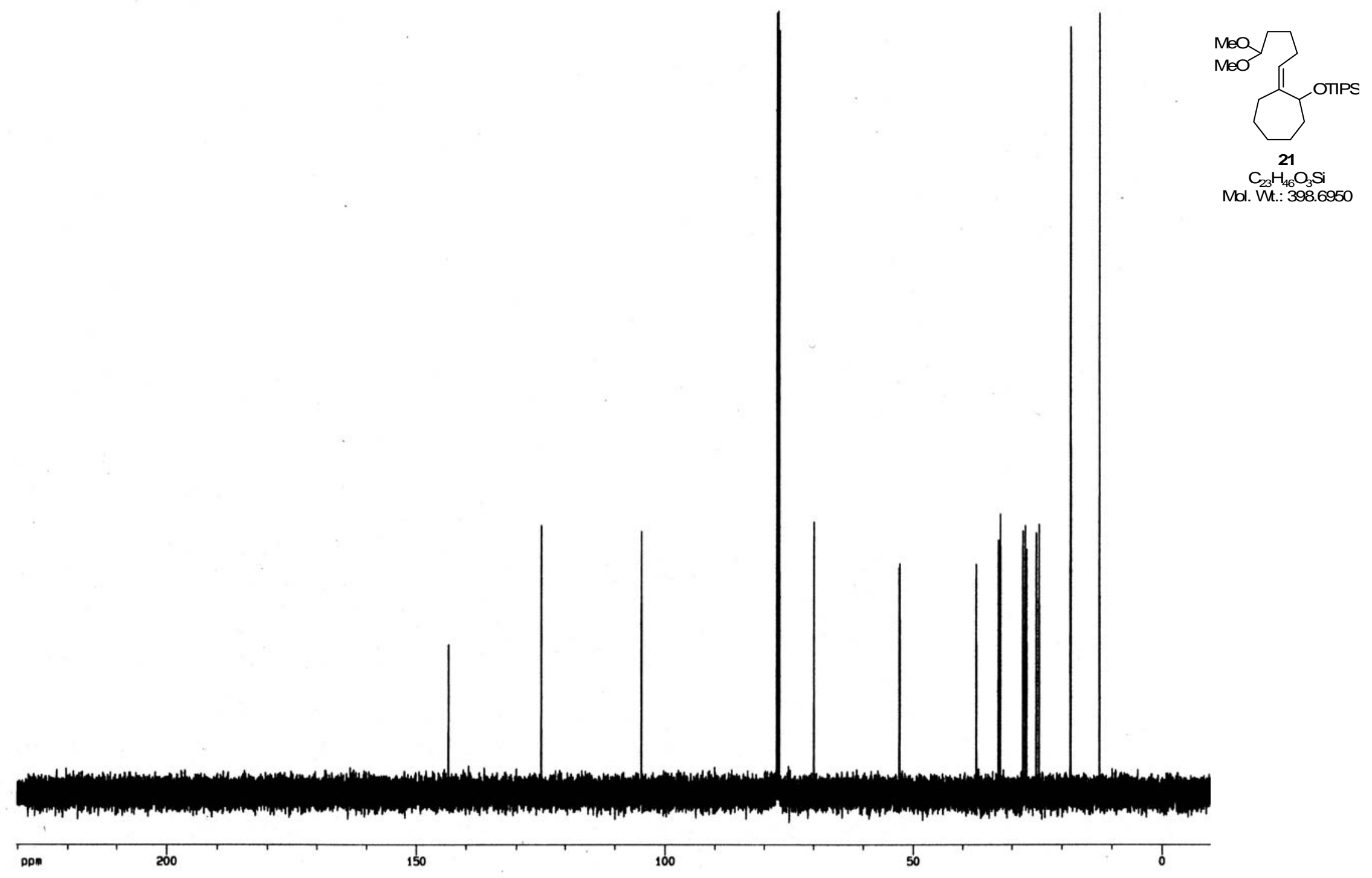




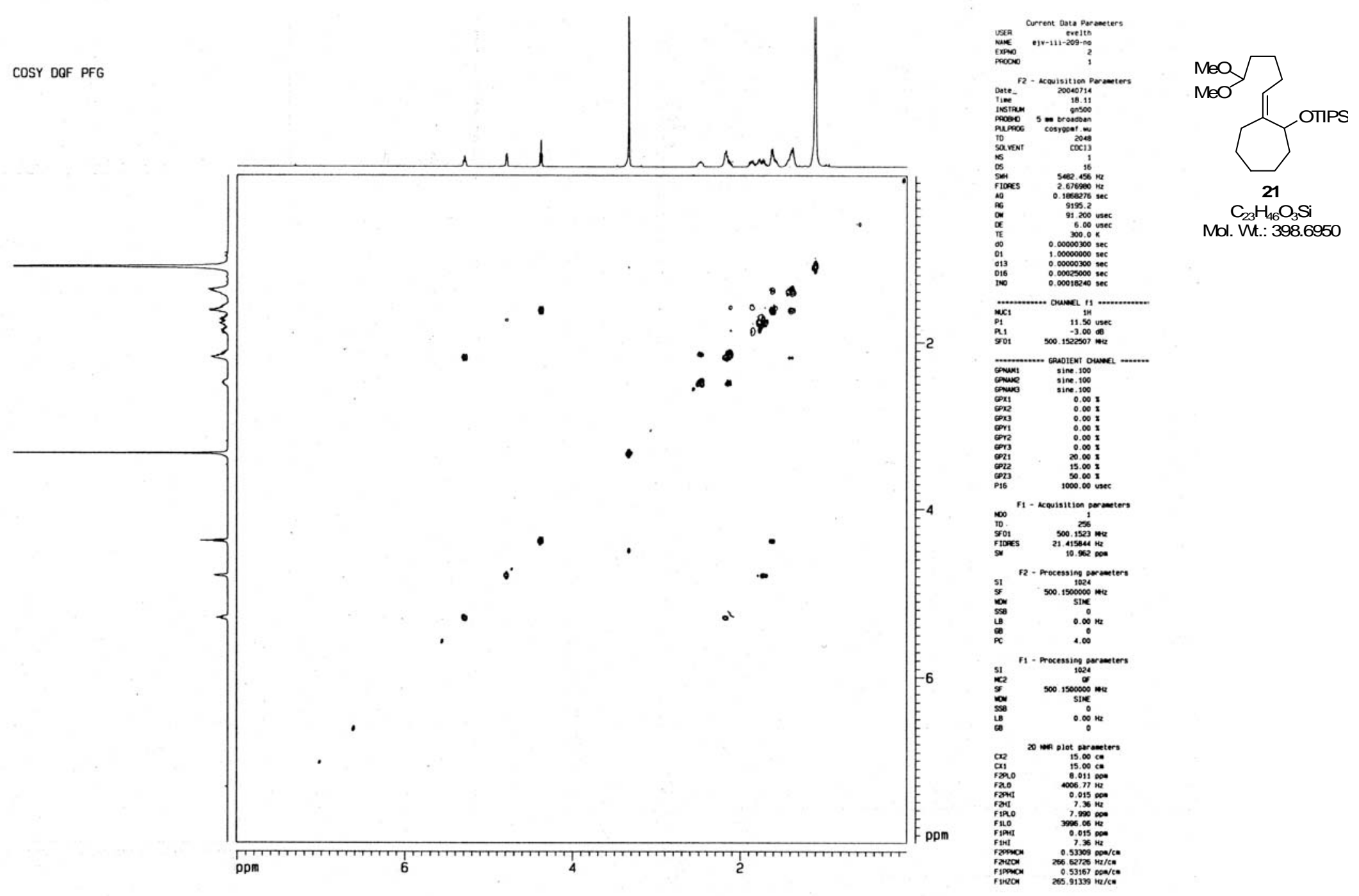




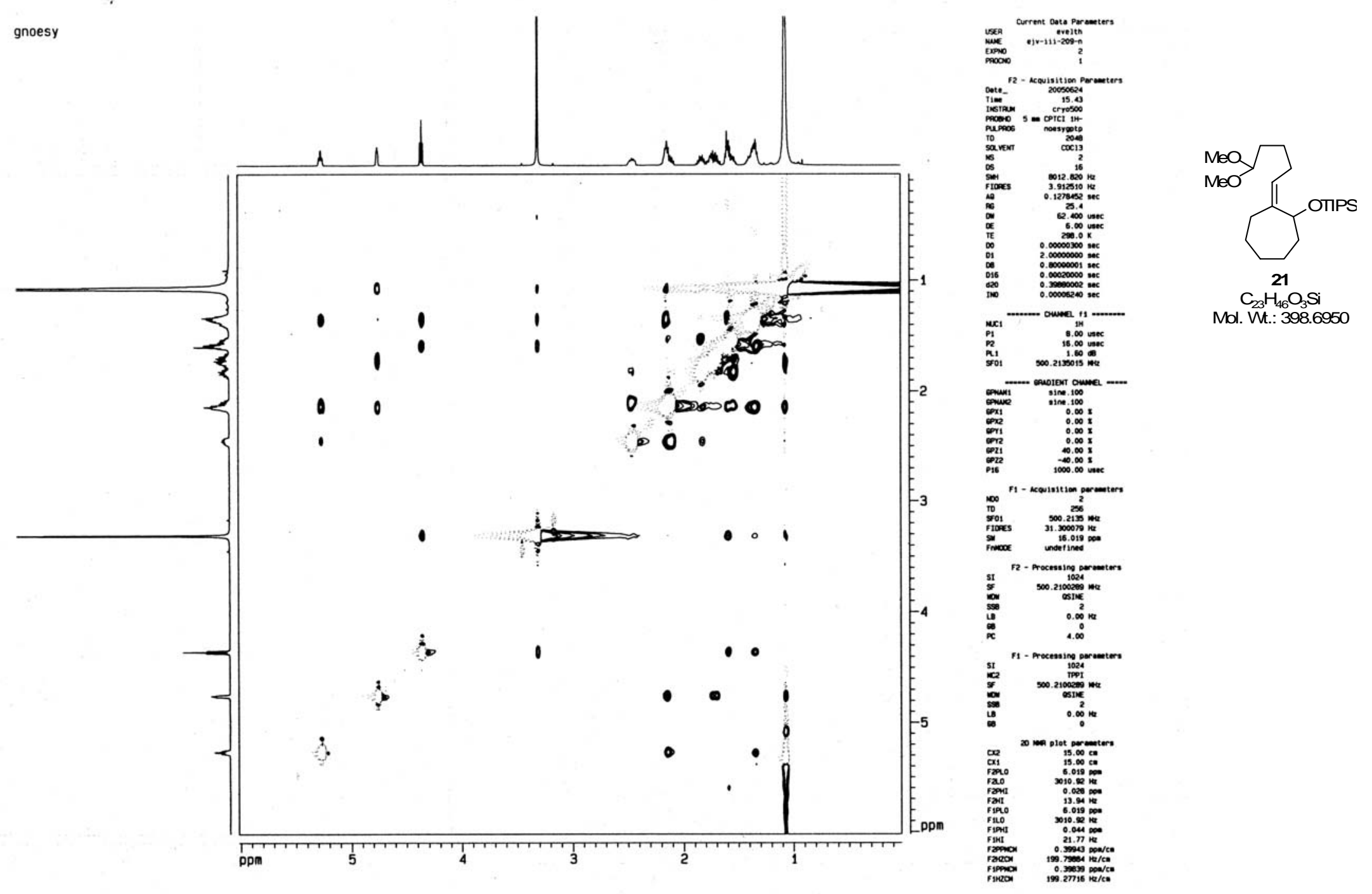




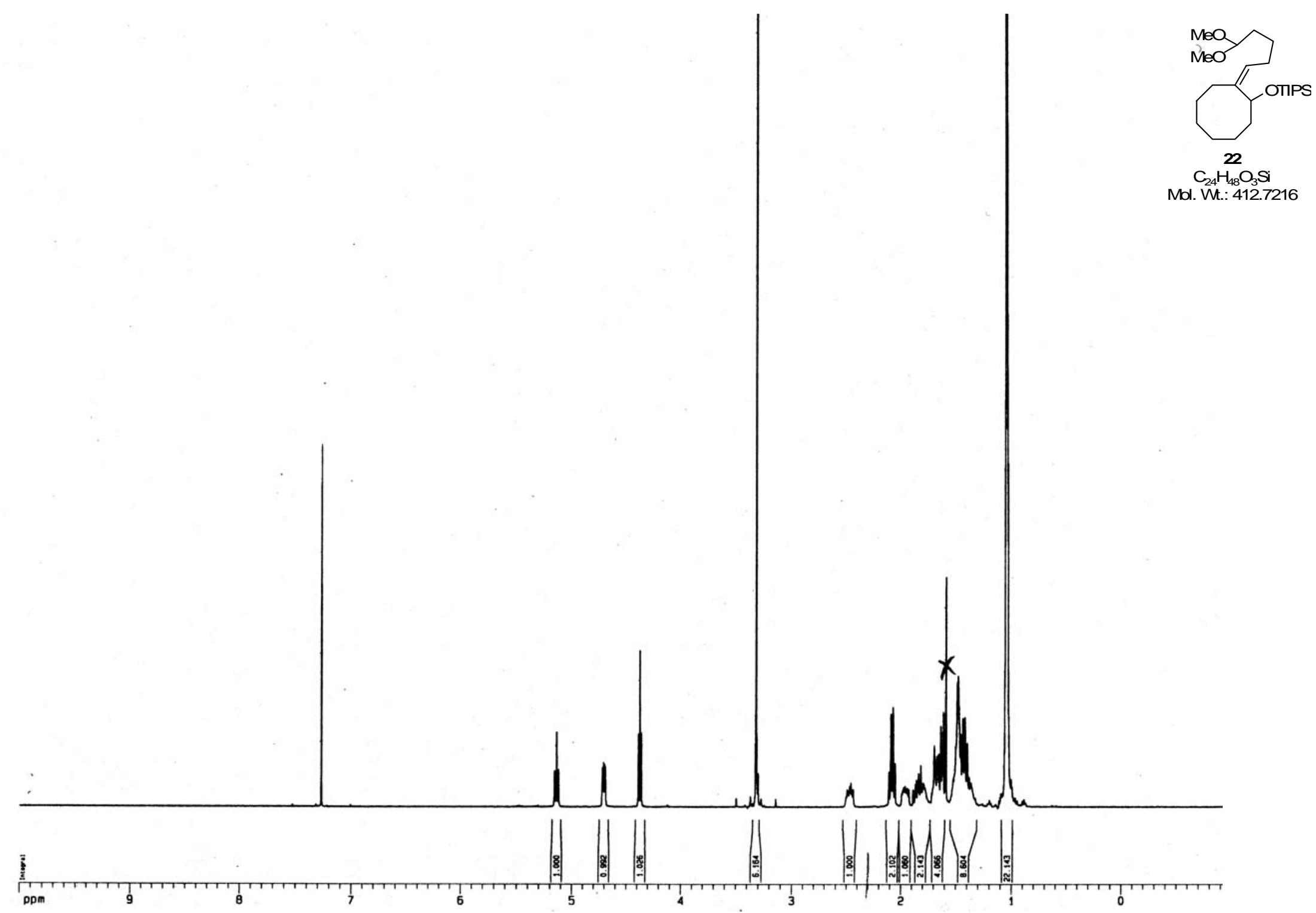




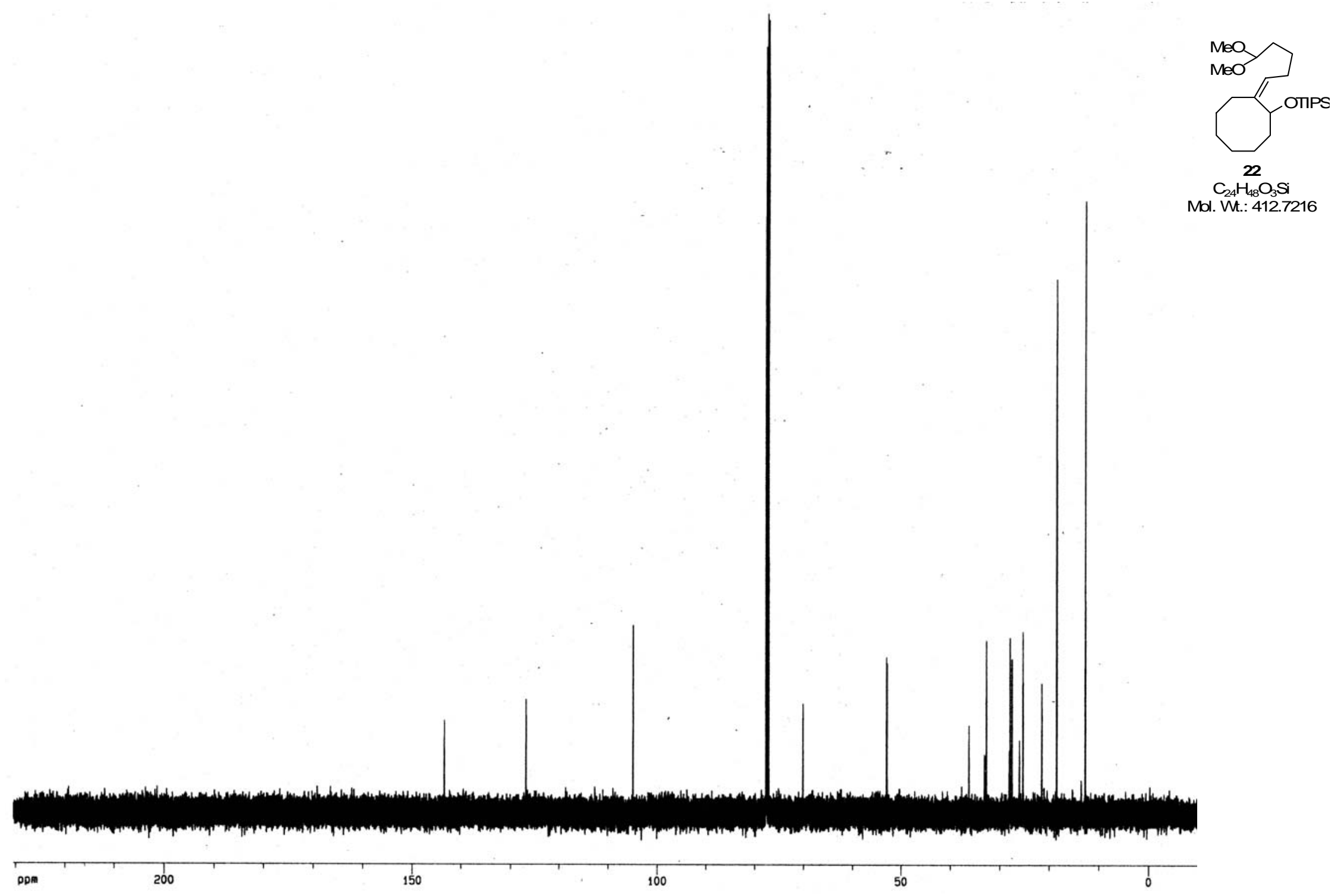




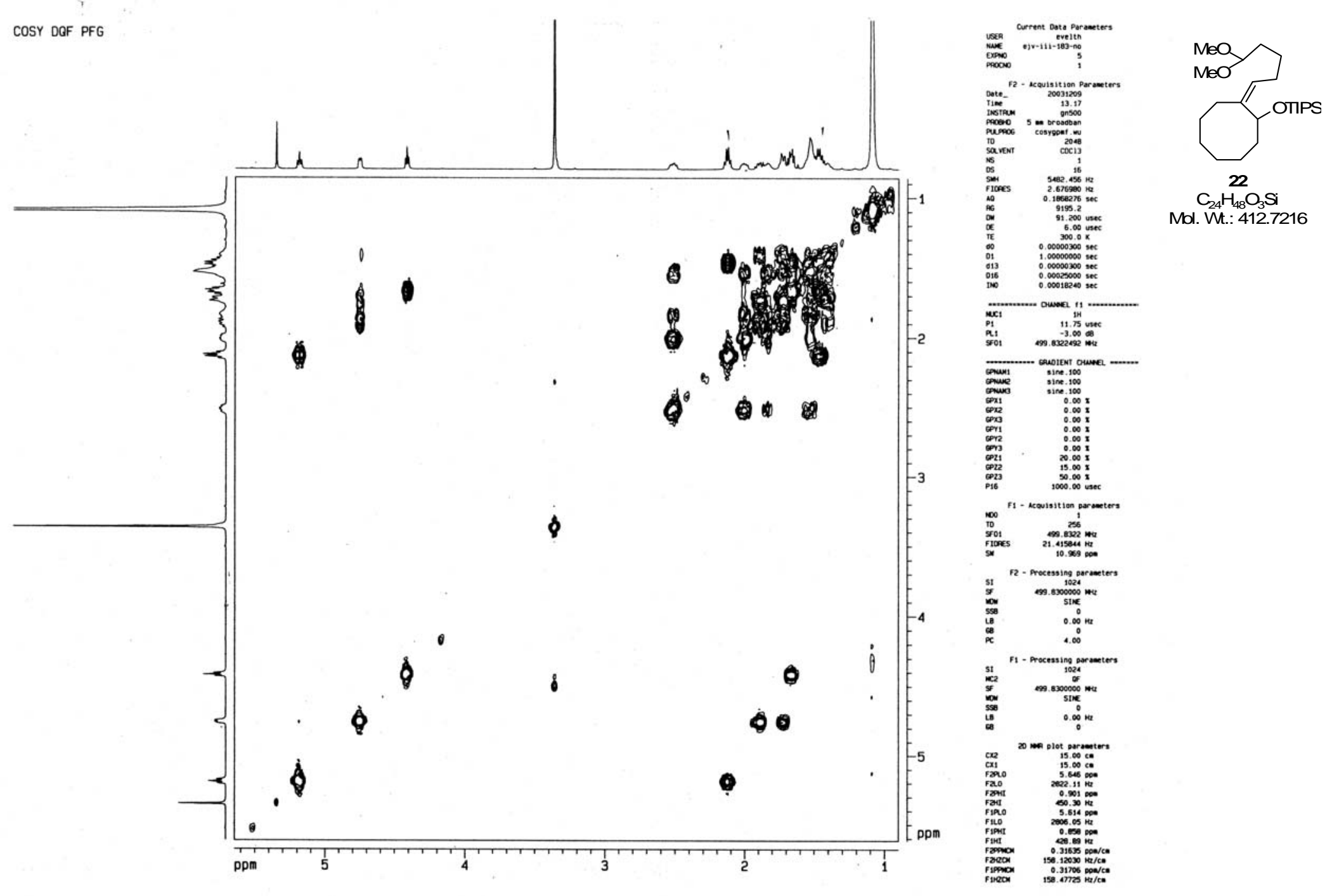




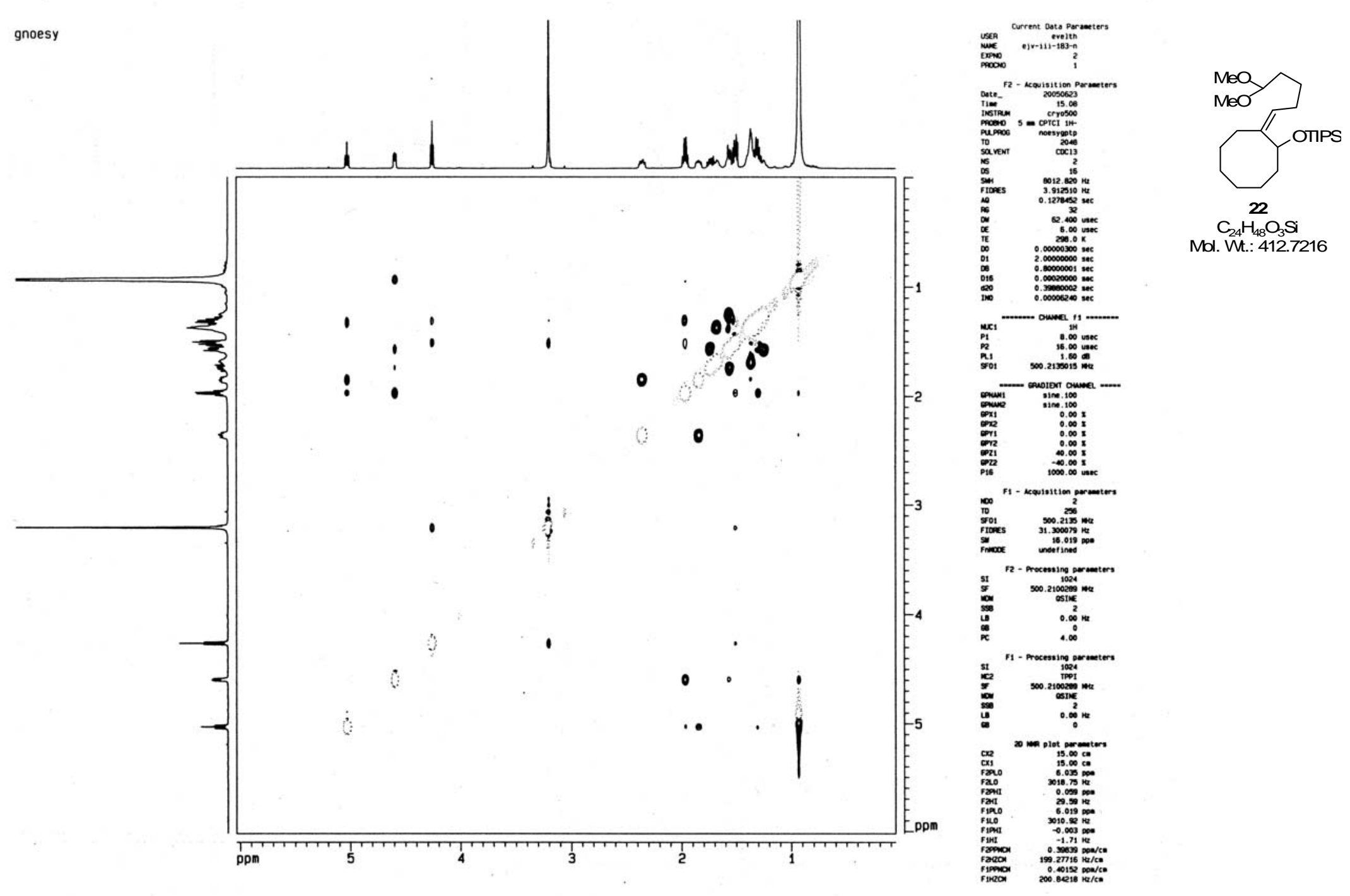




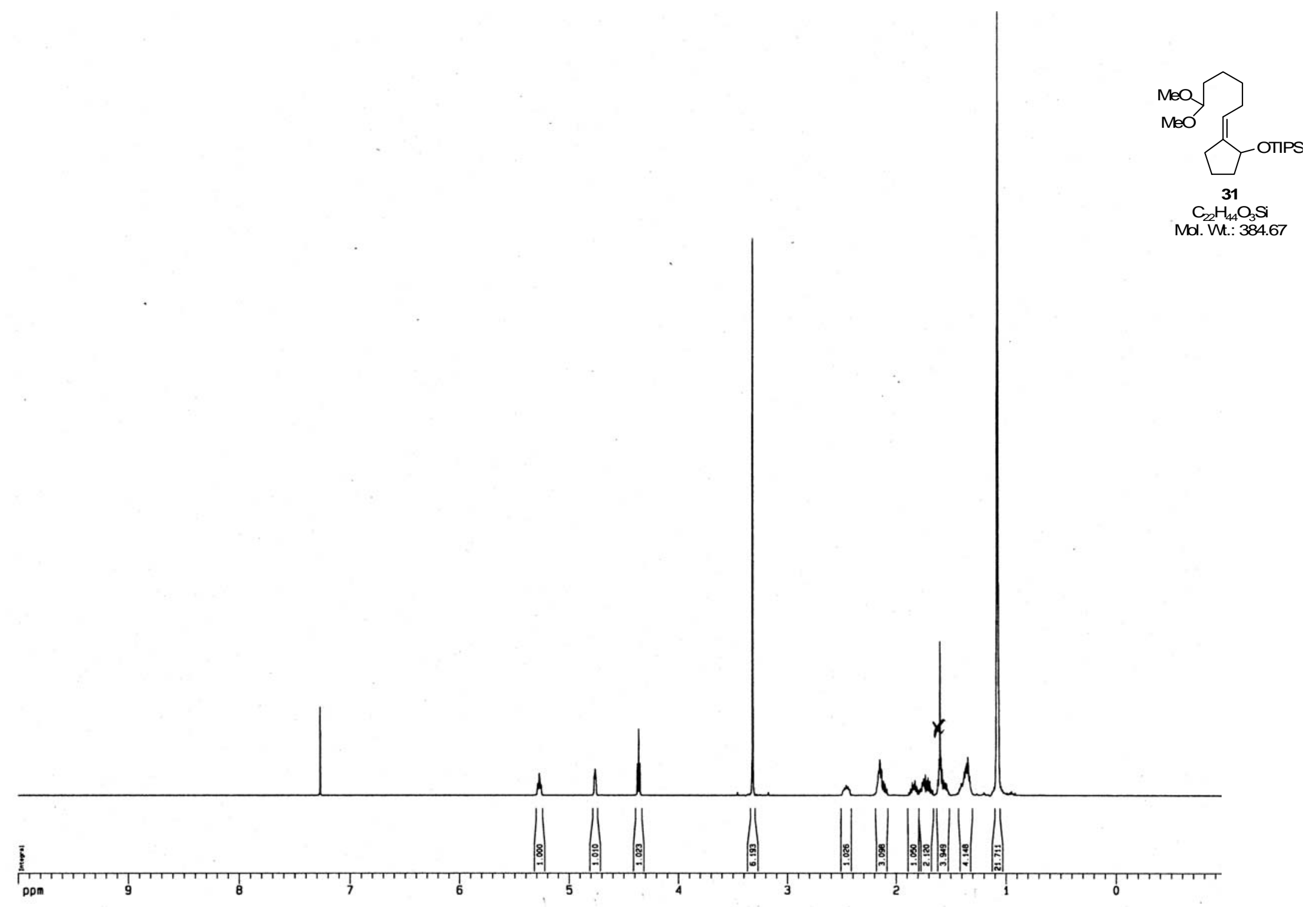




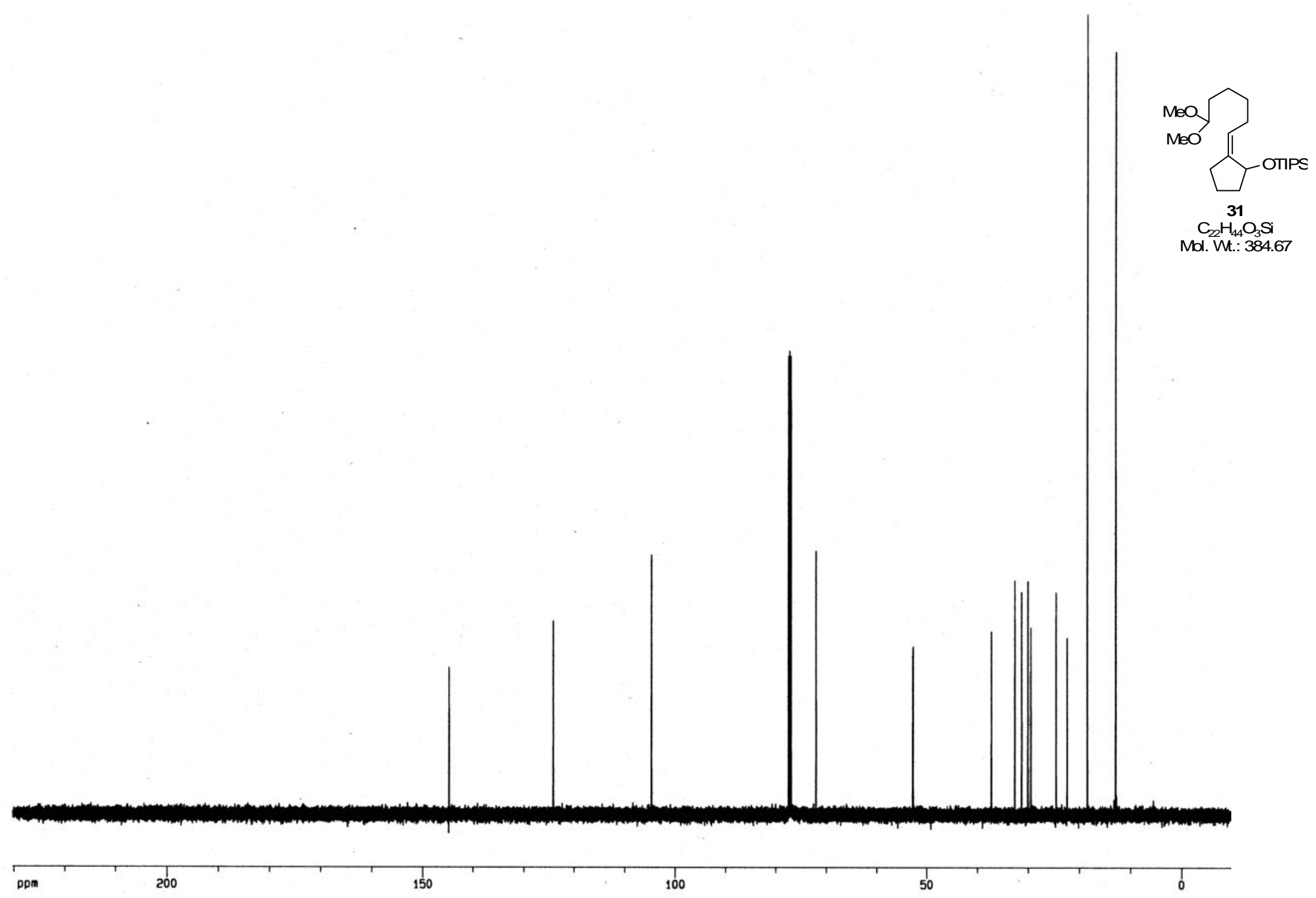




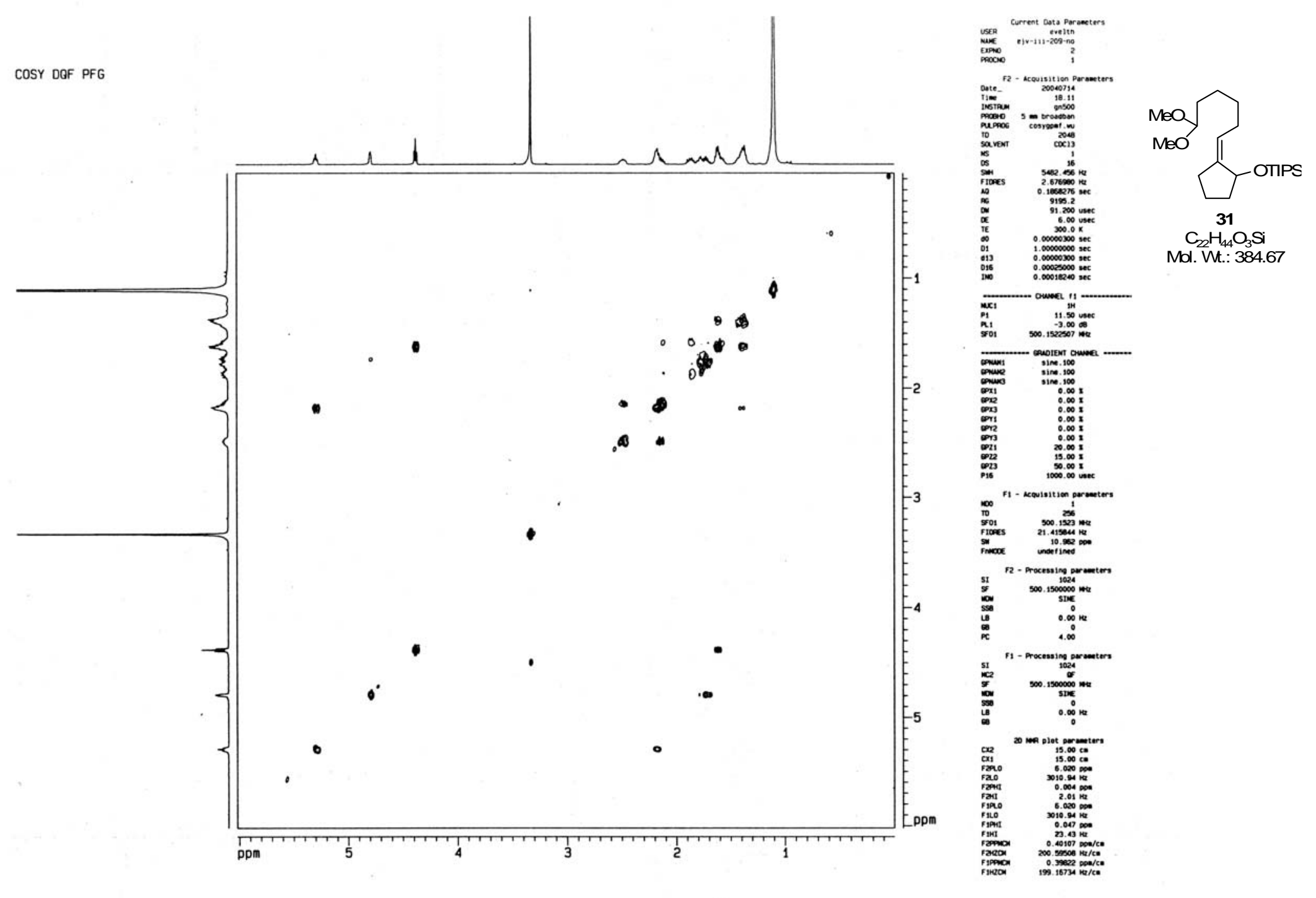




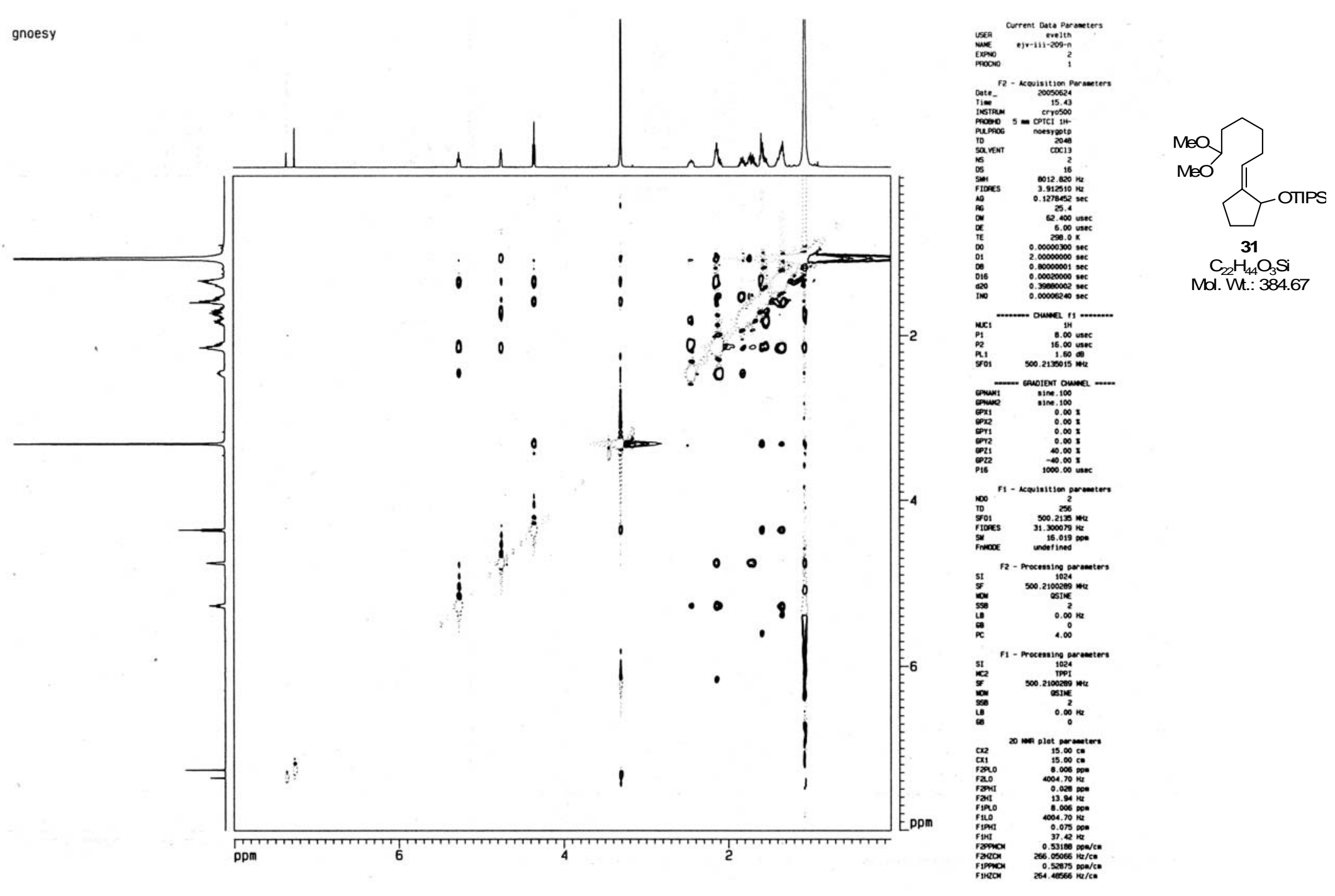




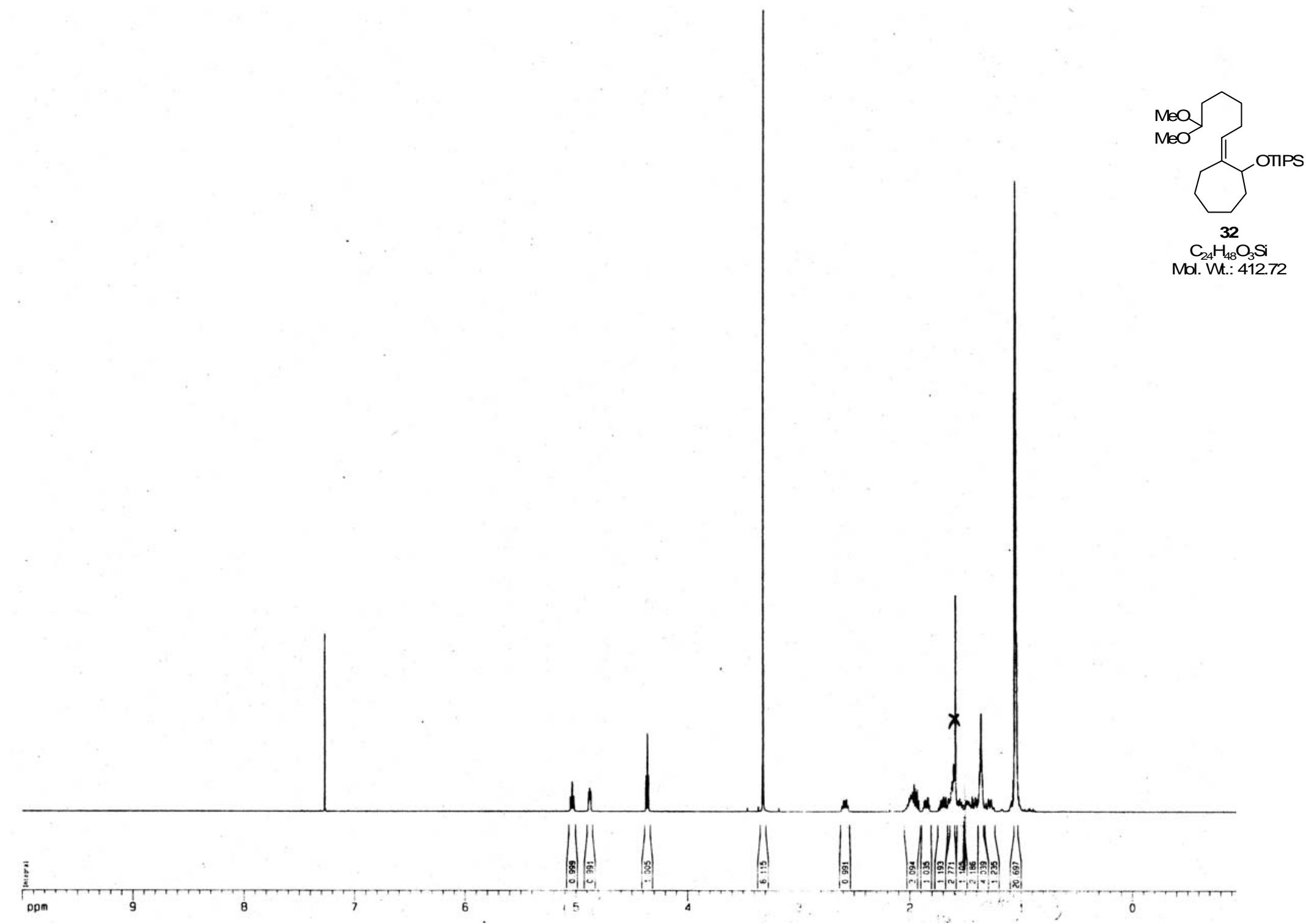




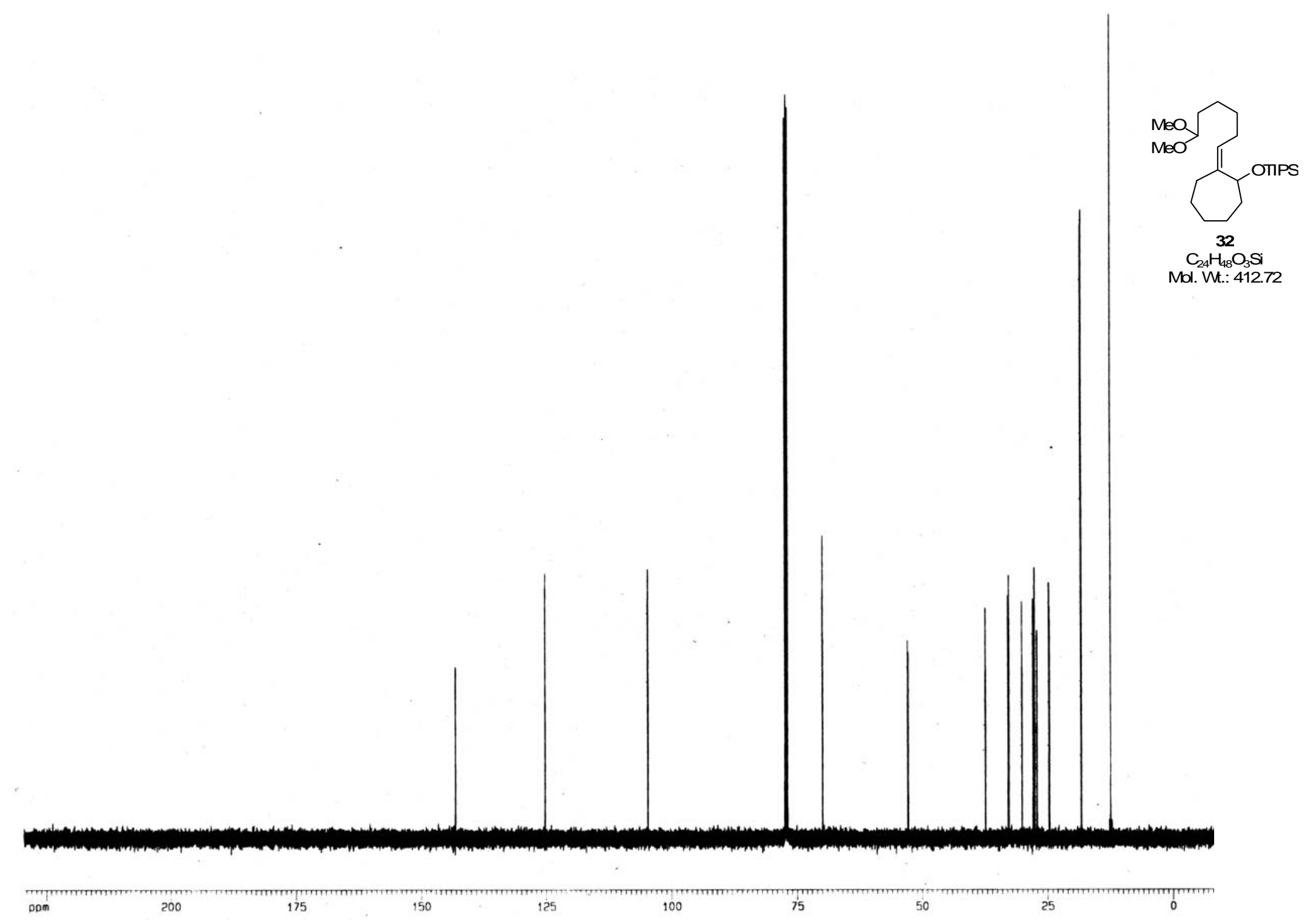




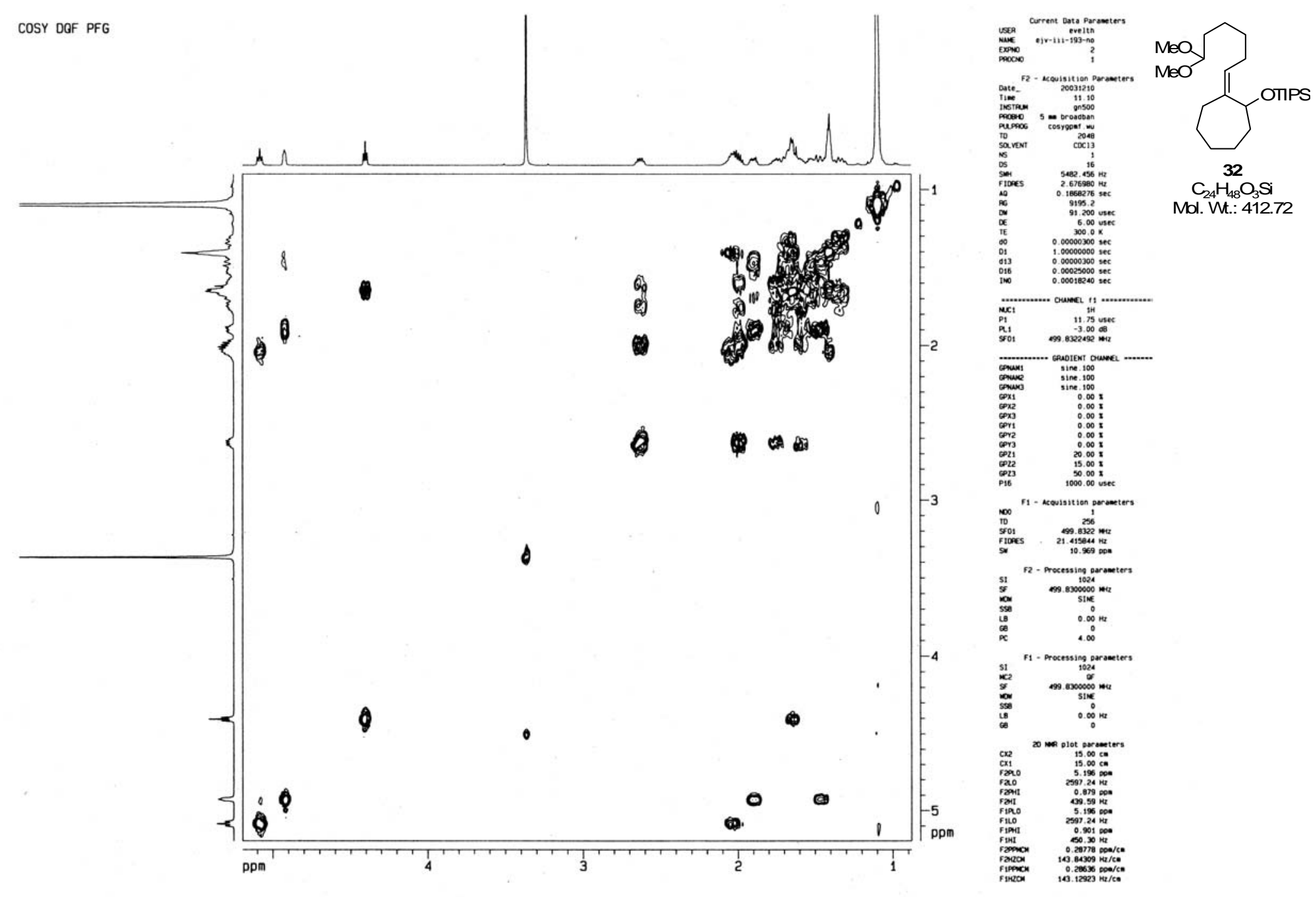




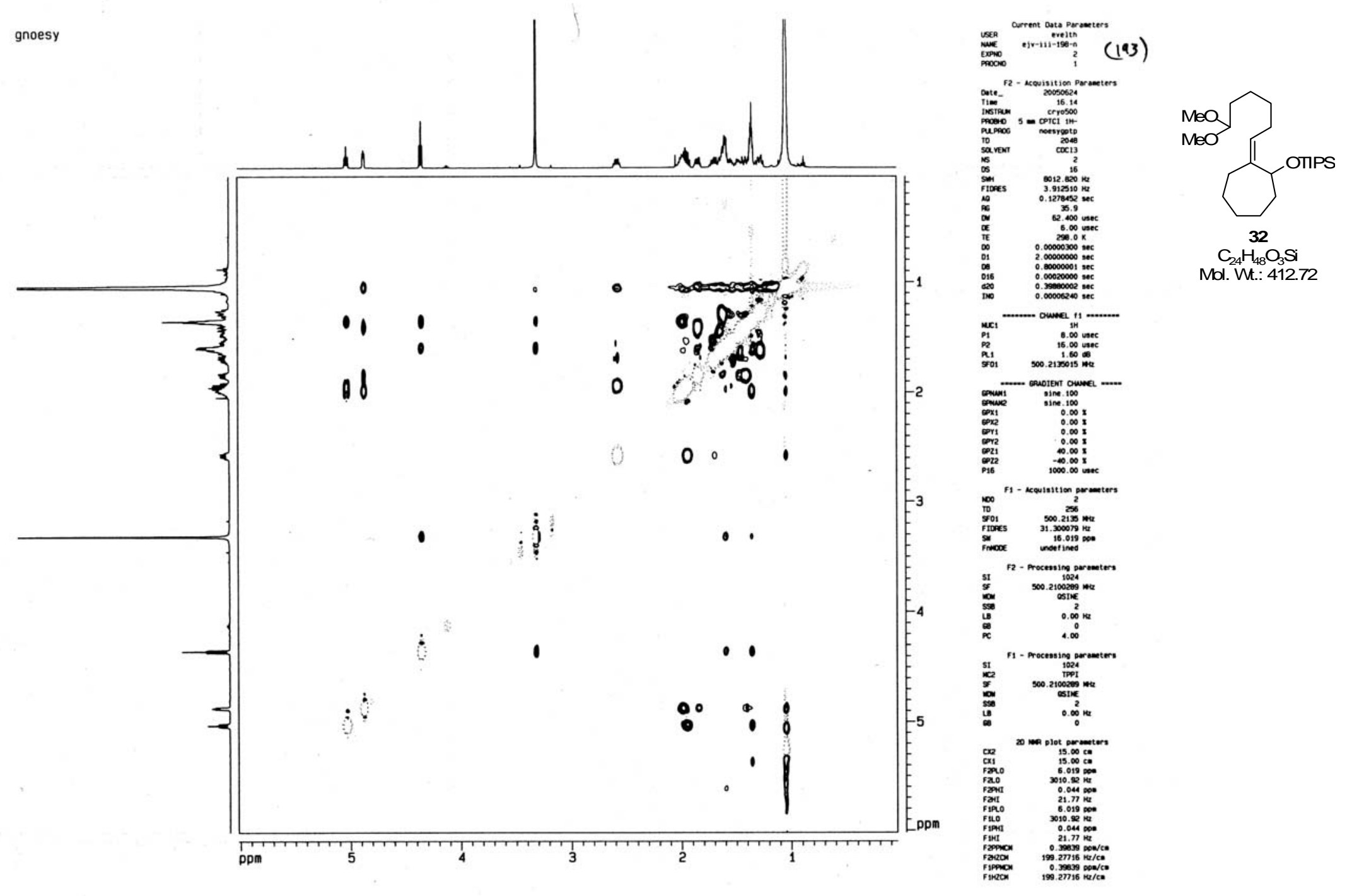




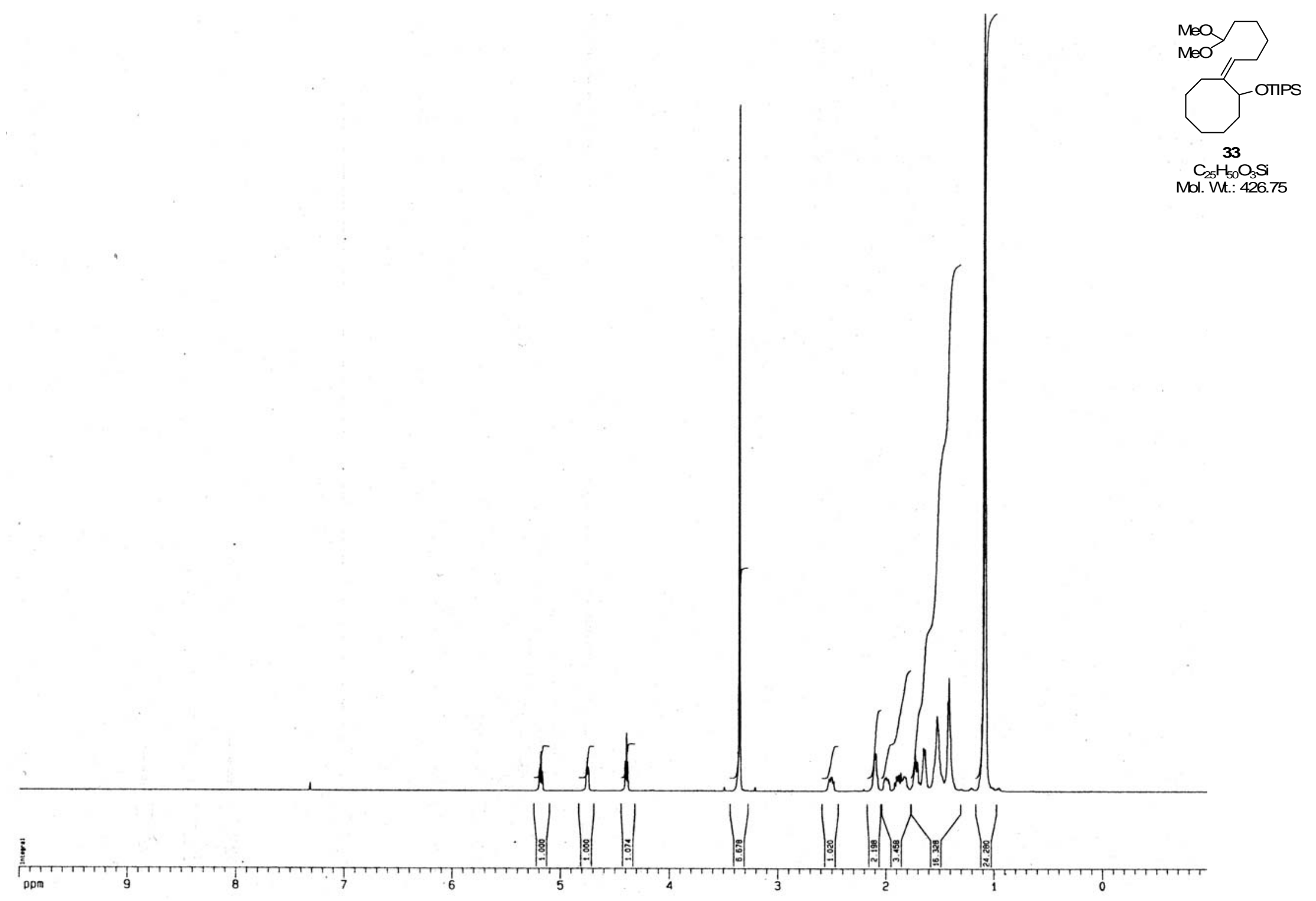




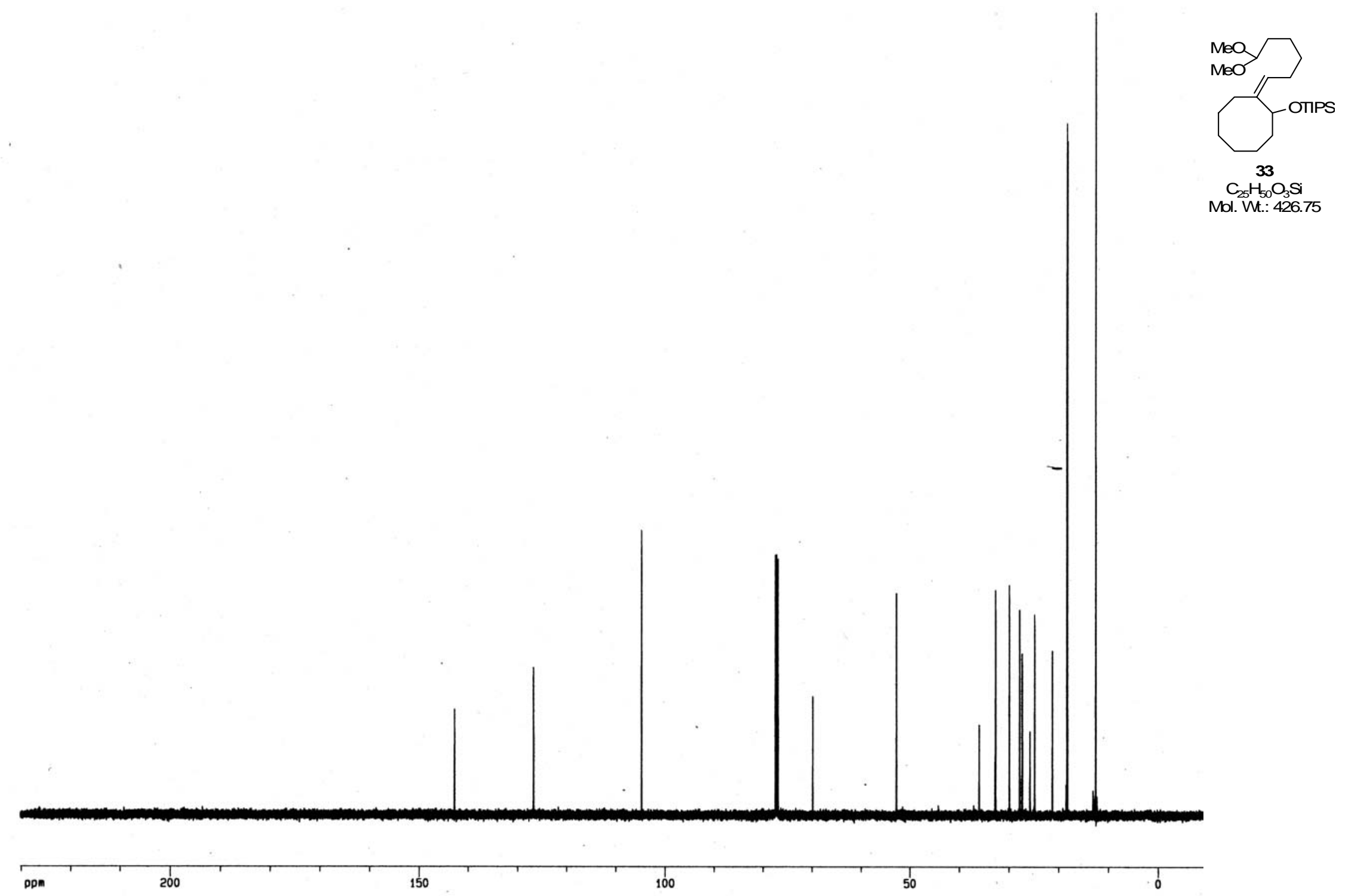




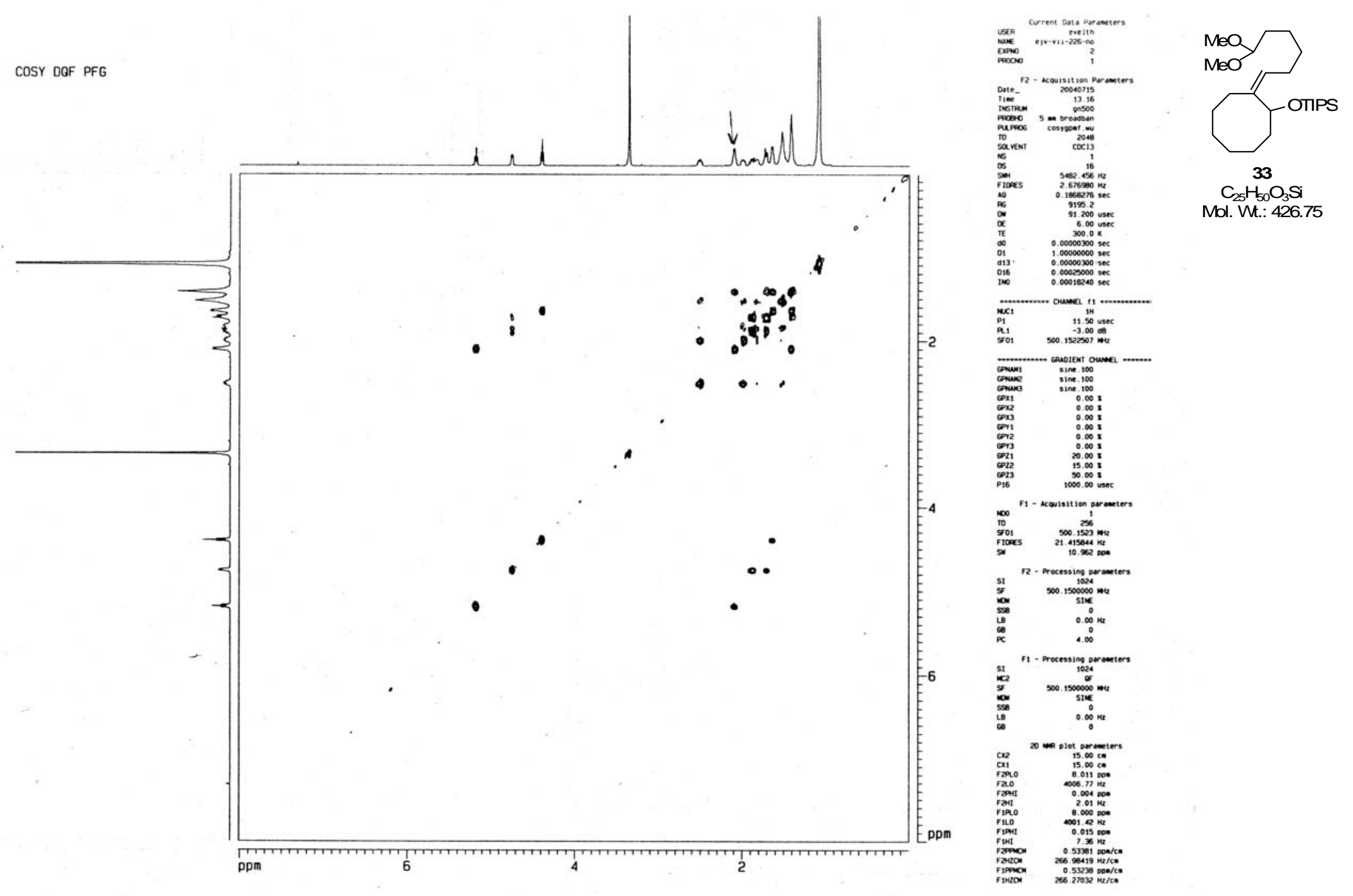



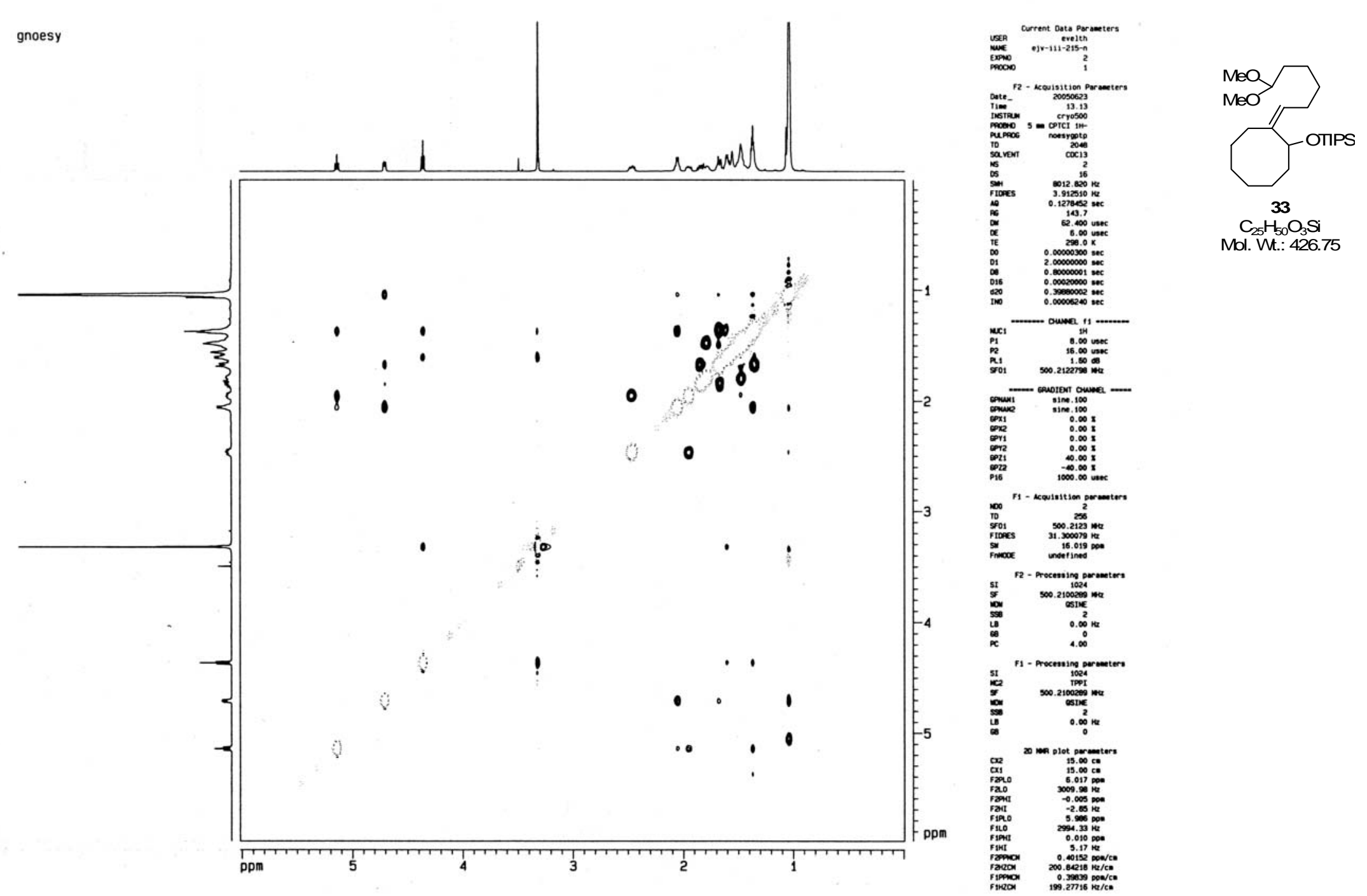


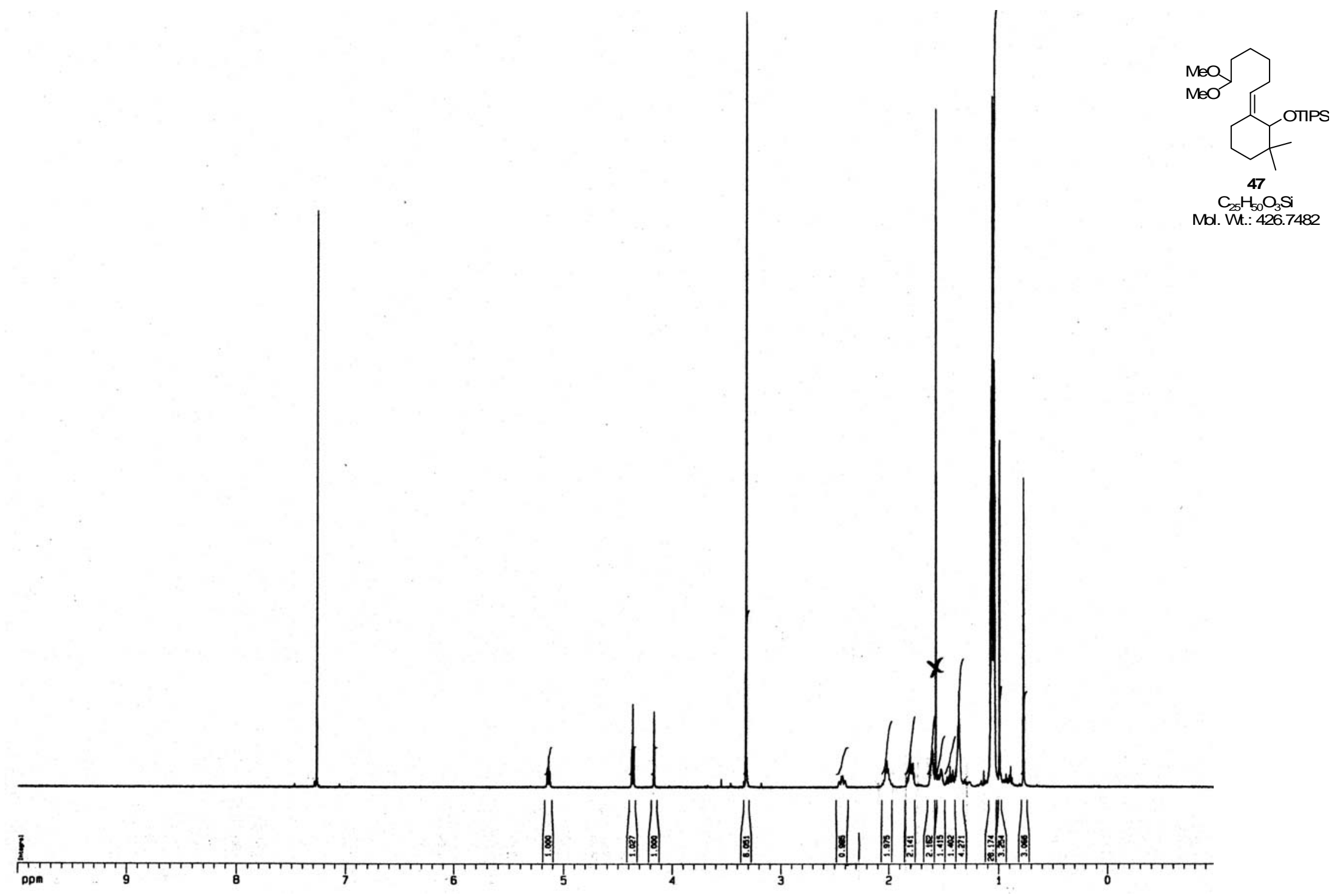




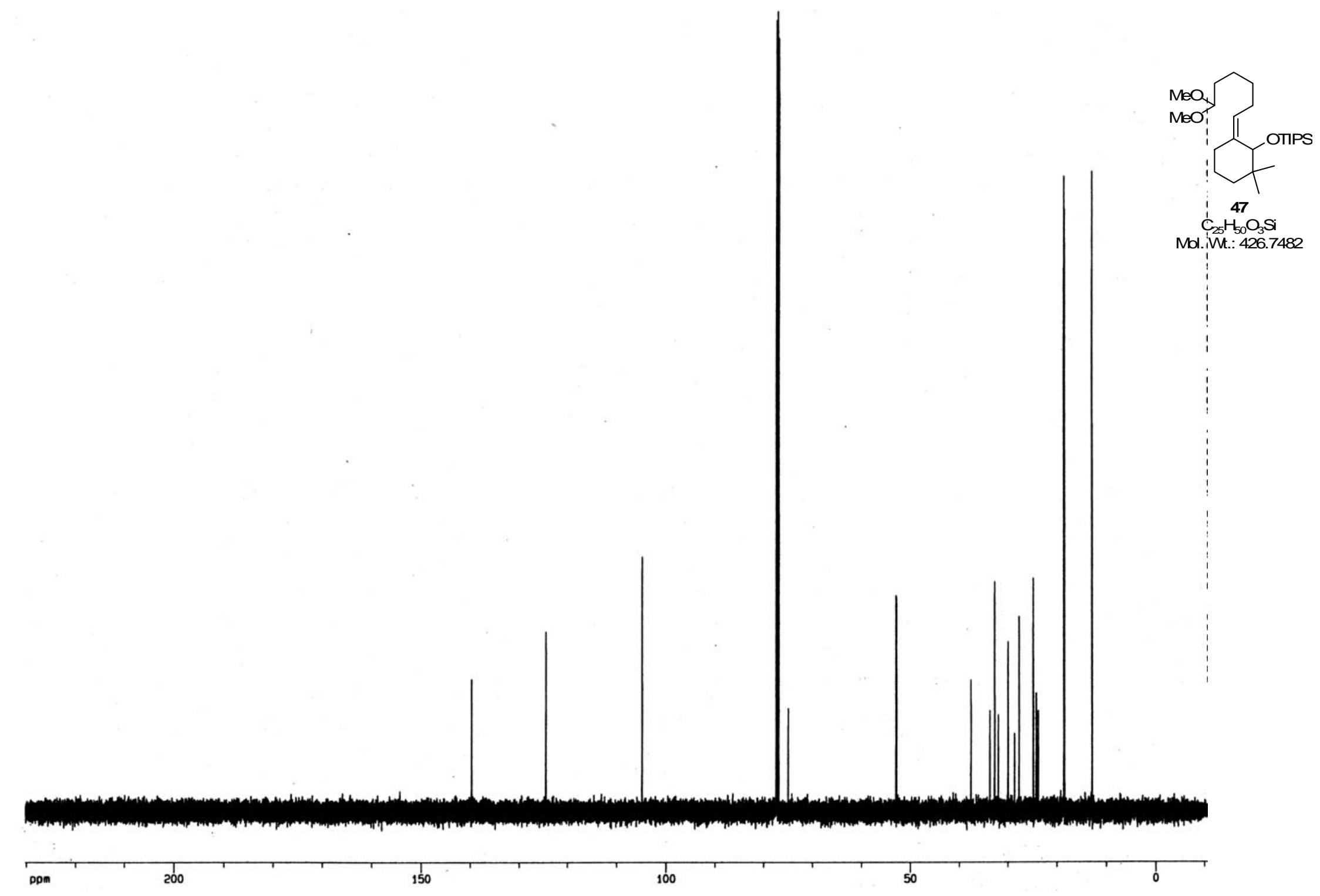



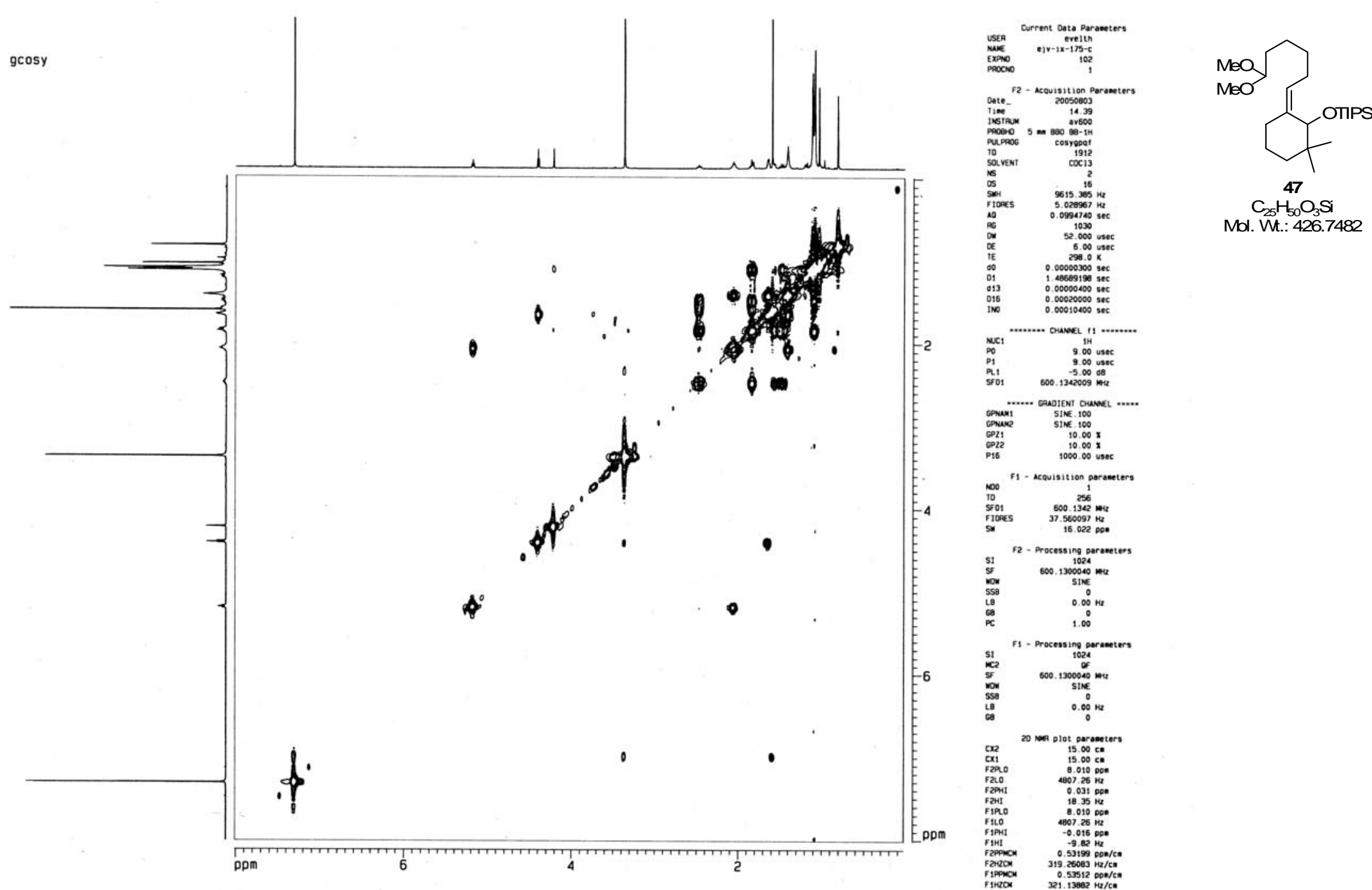

Mol. W.: 426.7482 


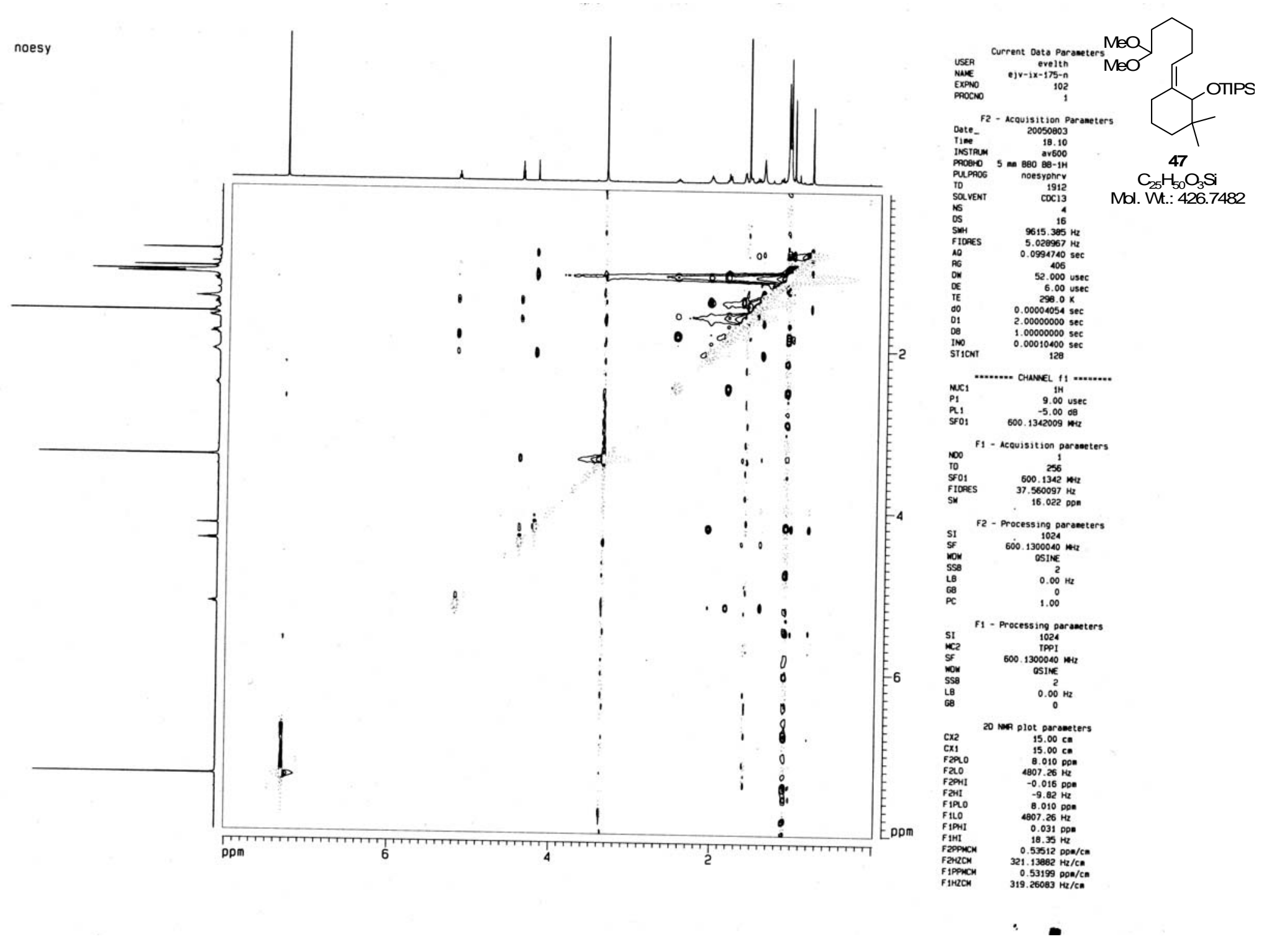




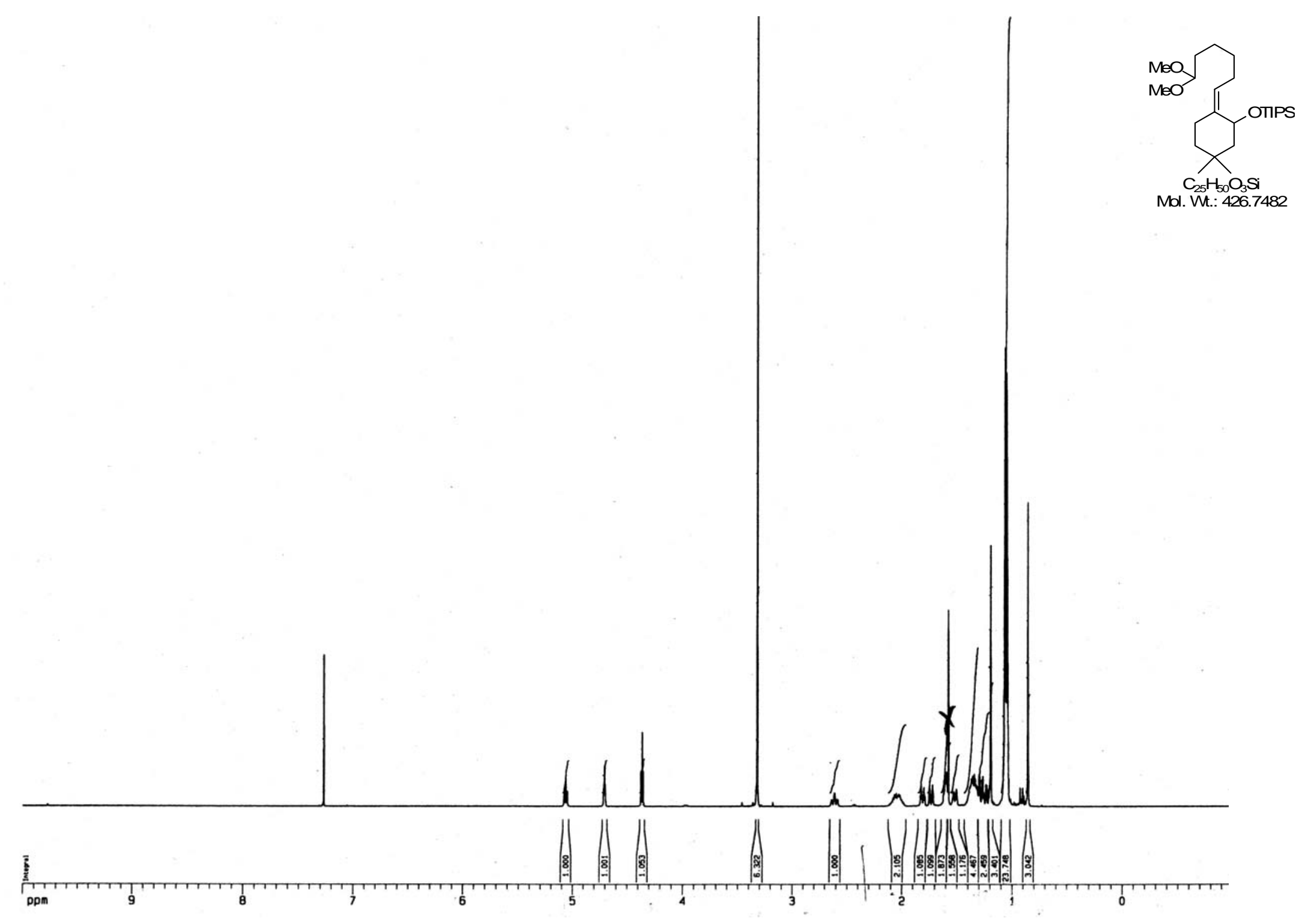




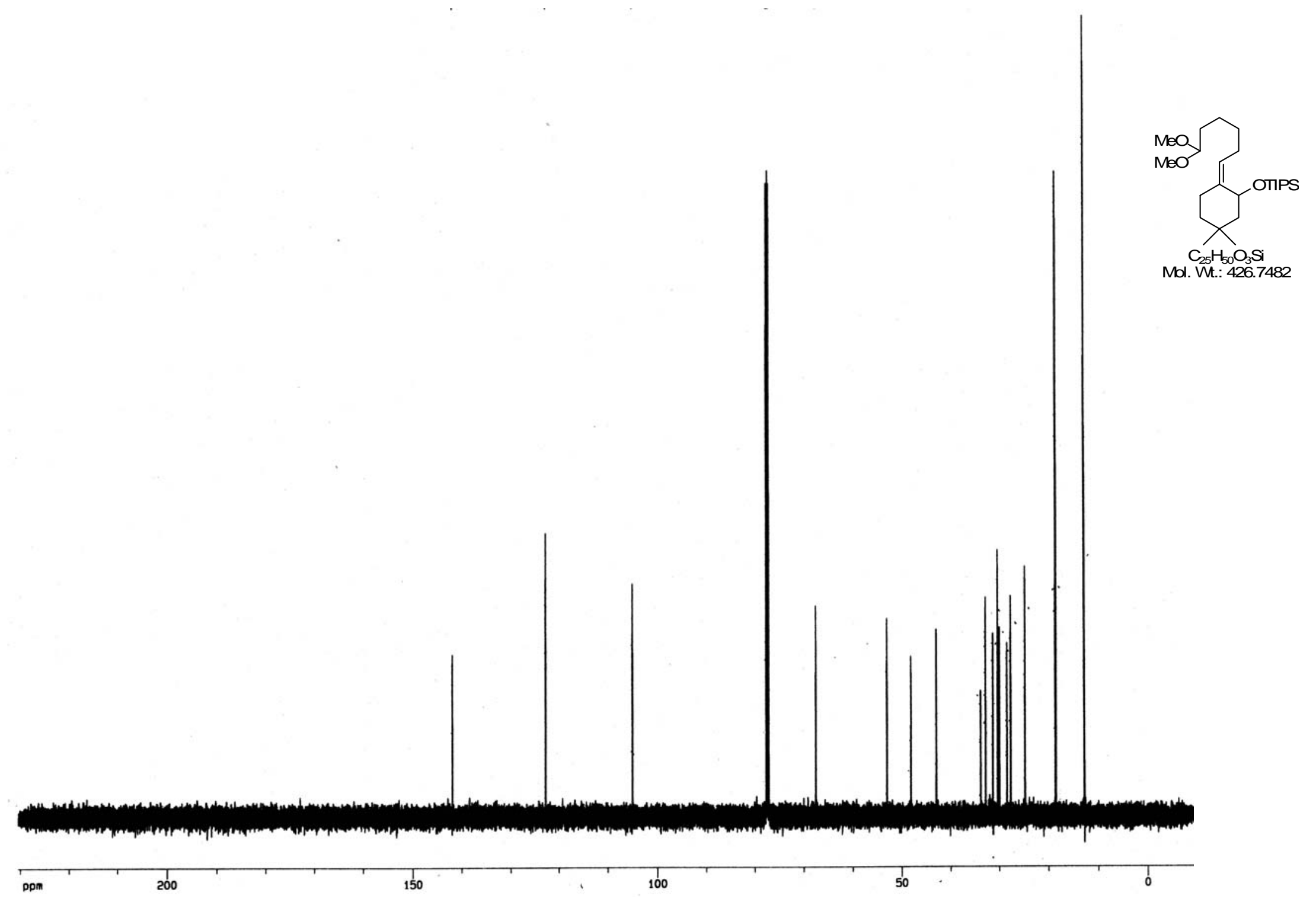




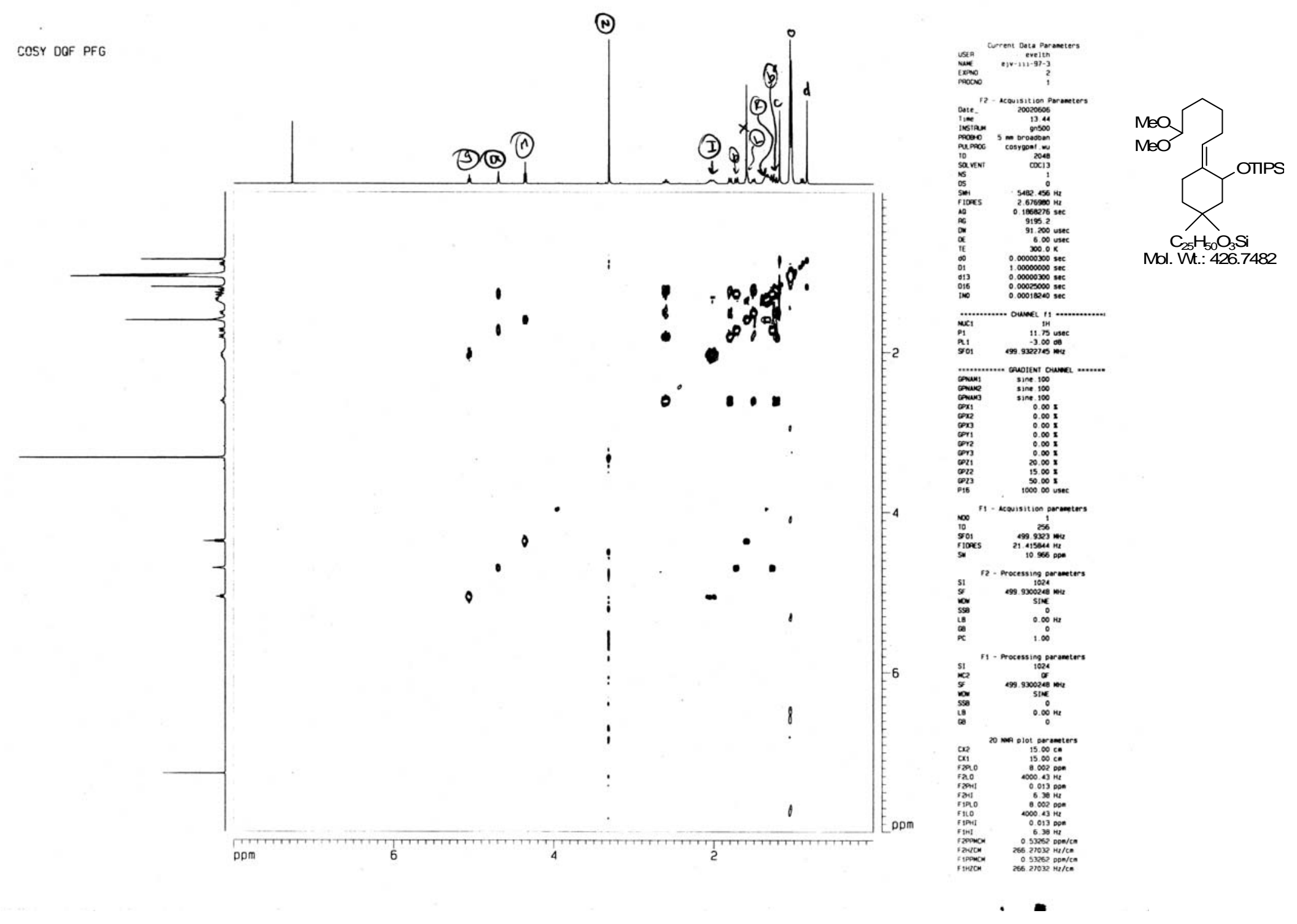




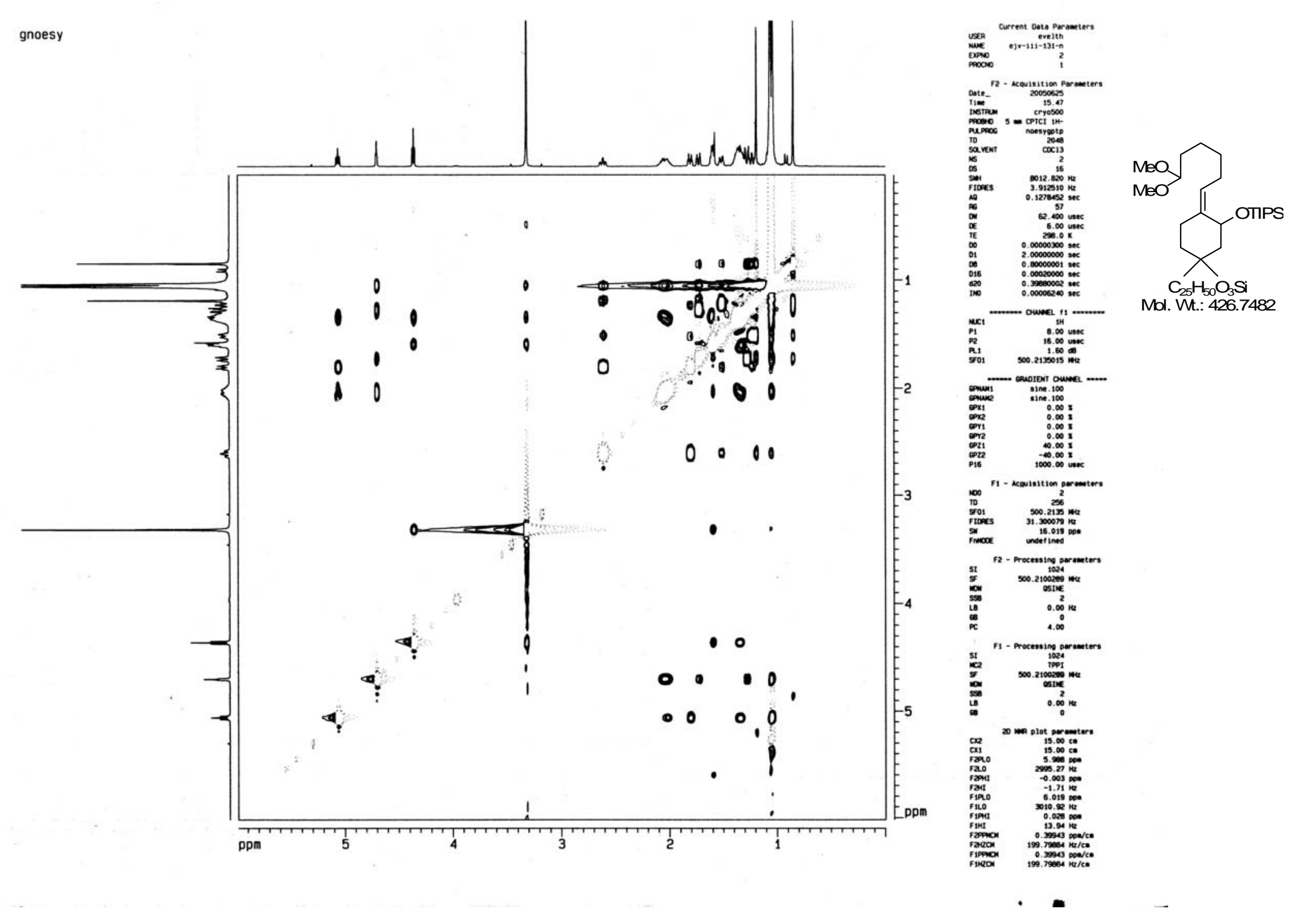




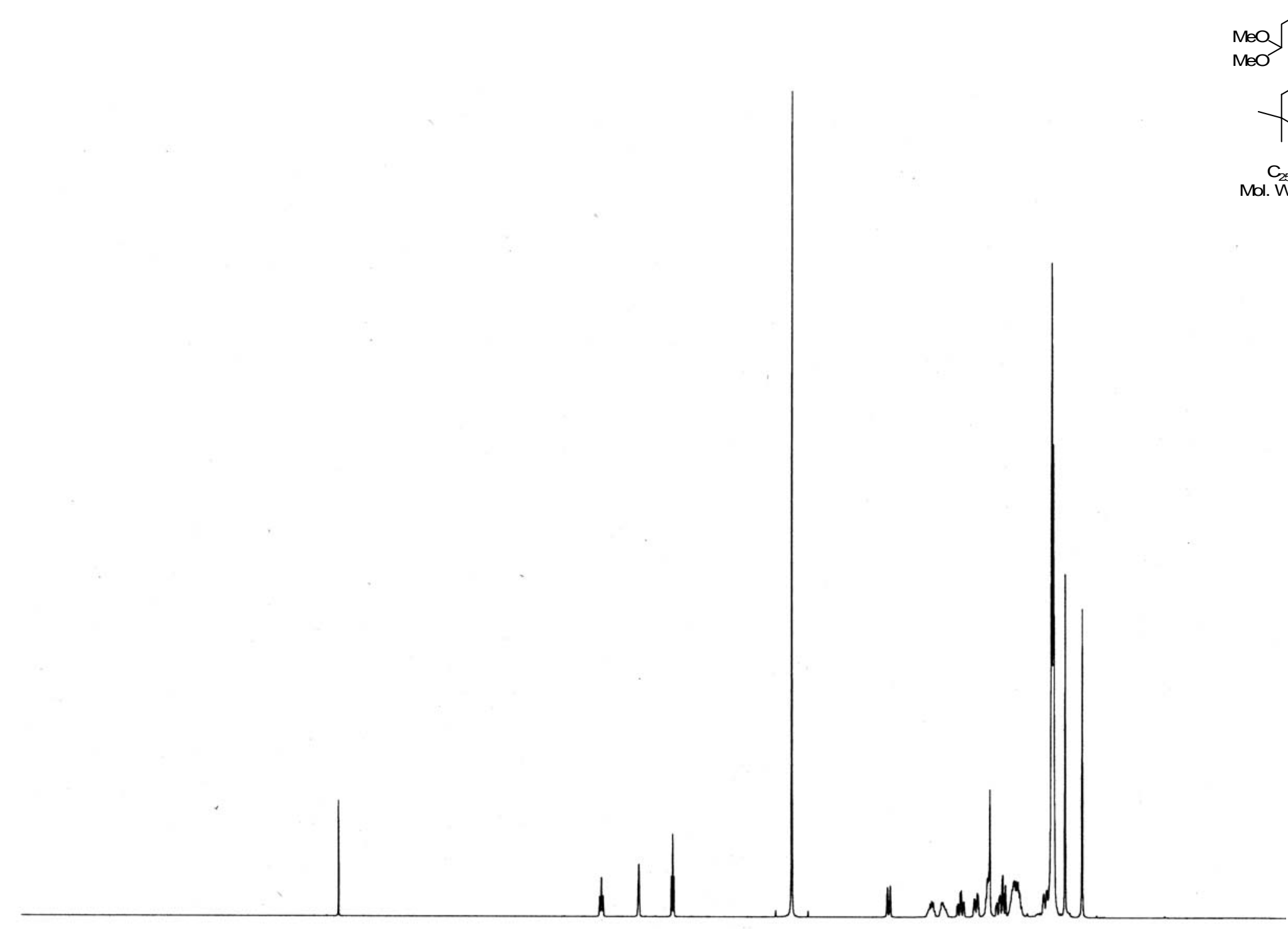




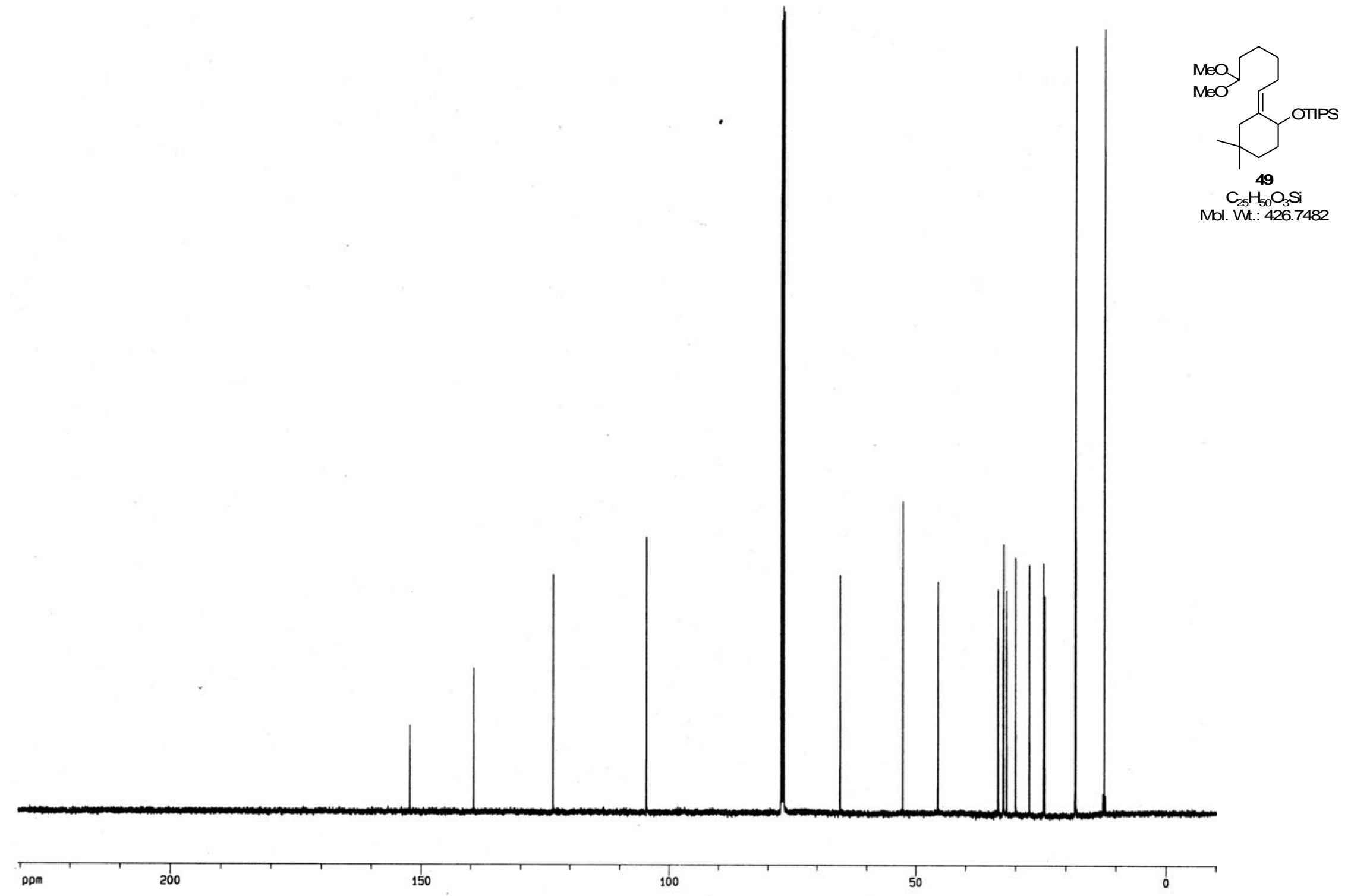




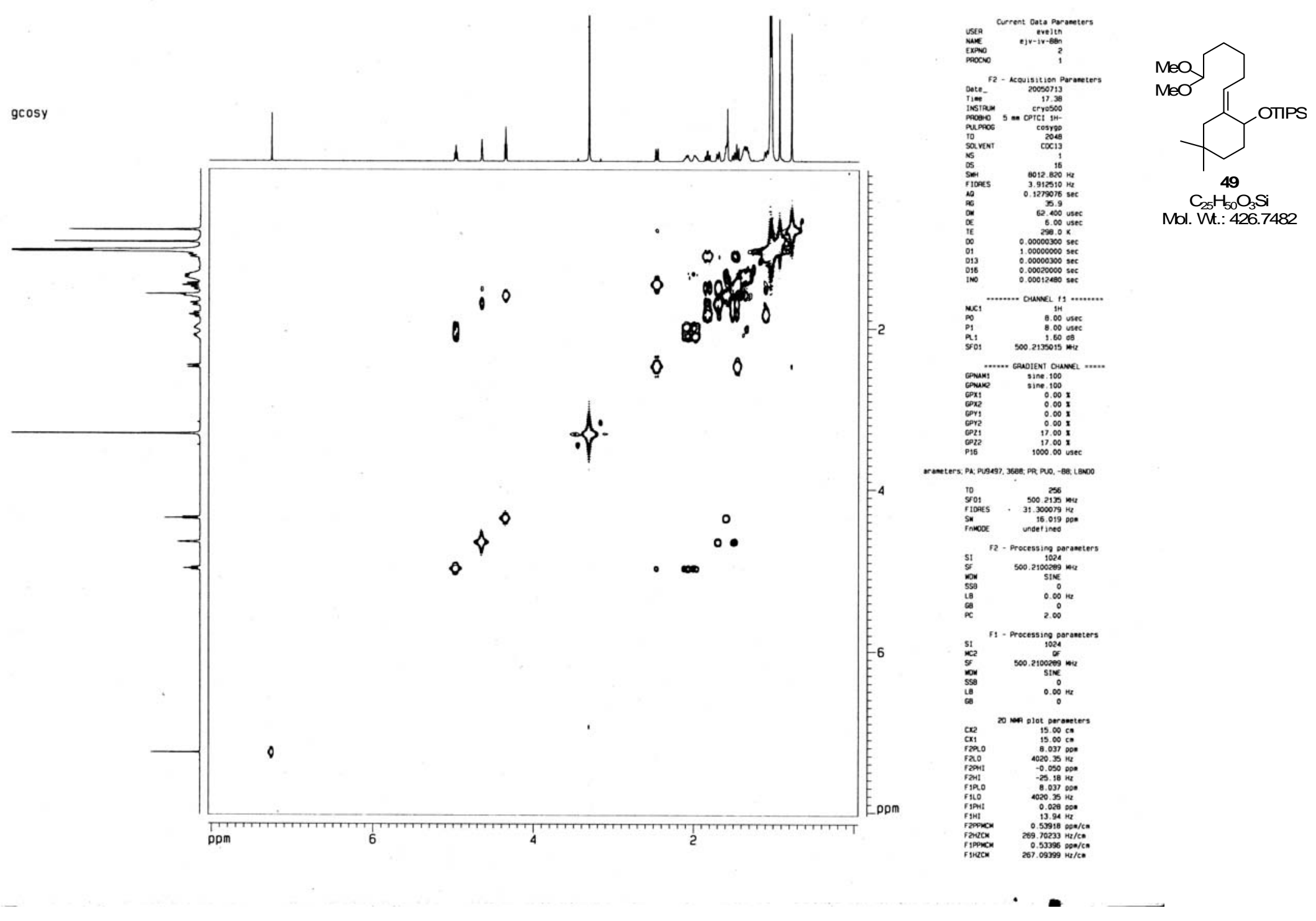



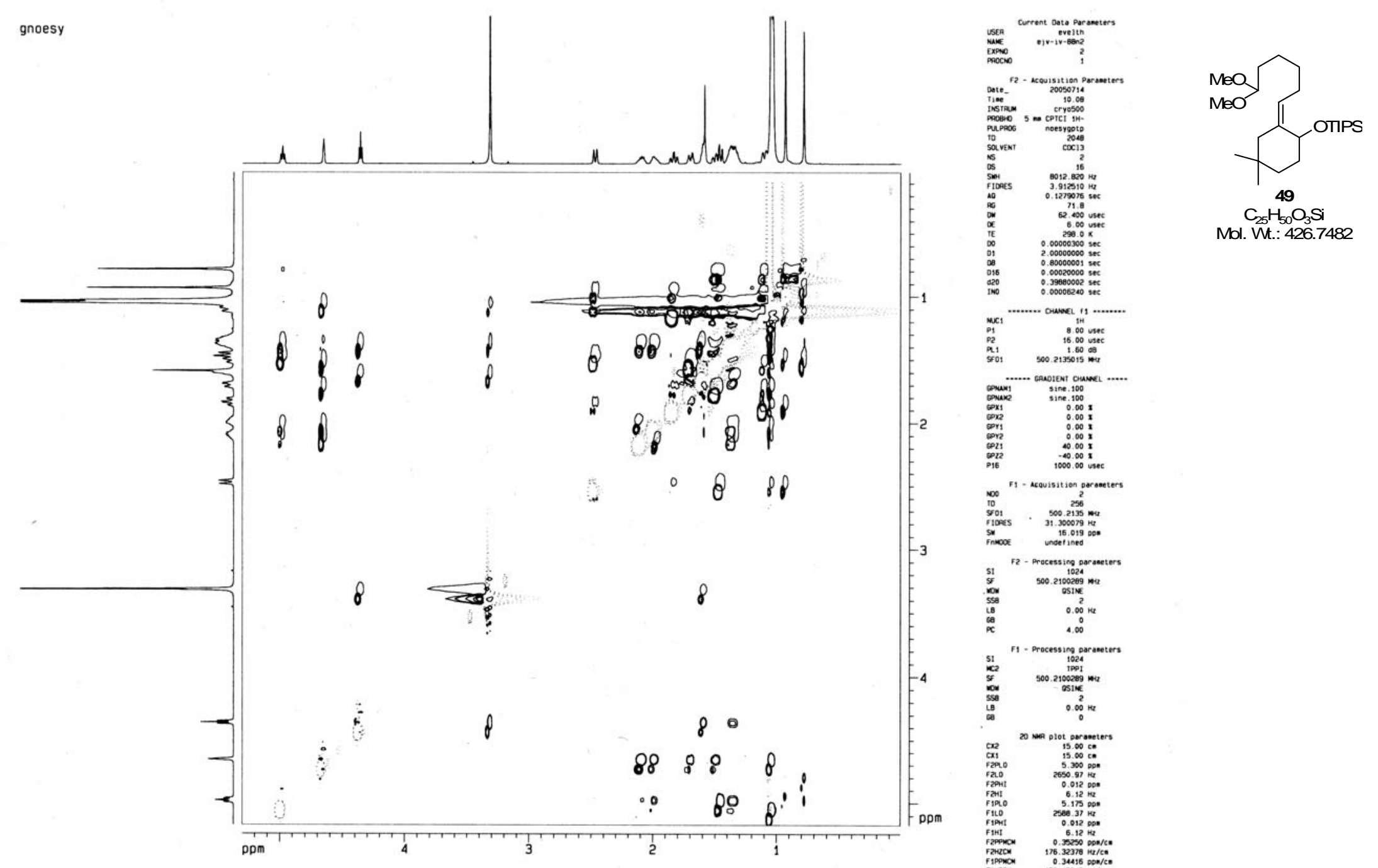

Mol. W..: 426.7482 


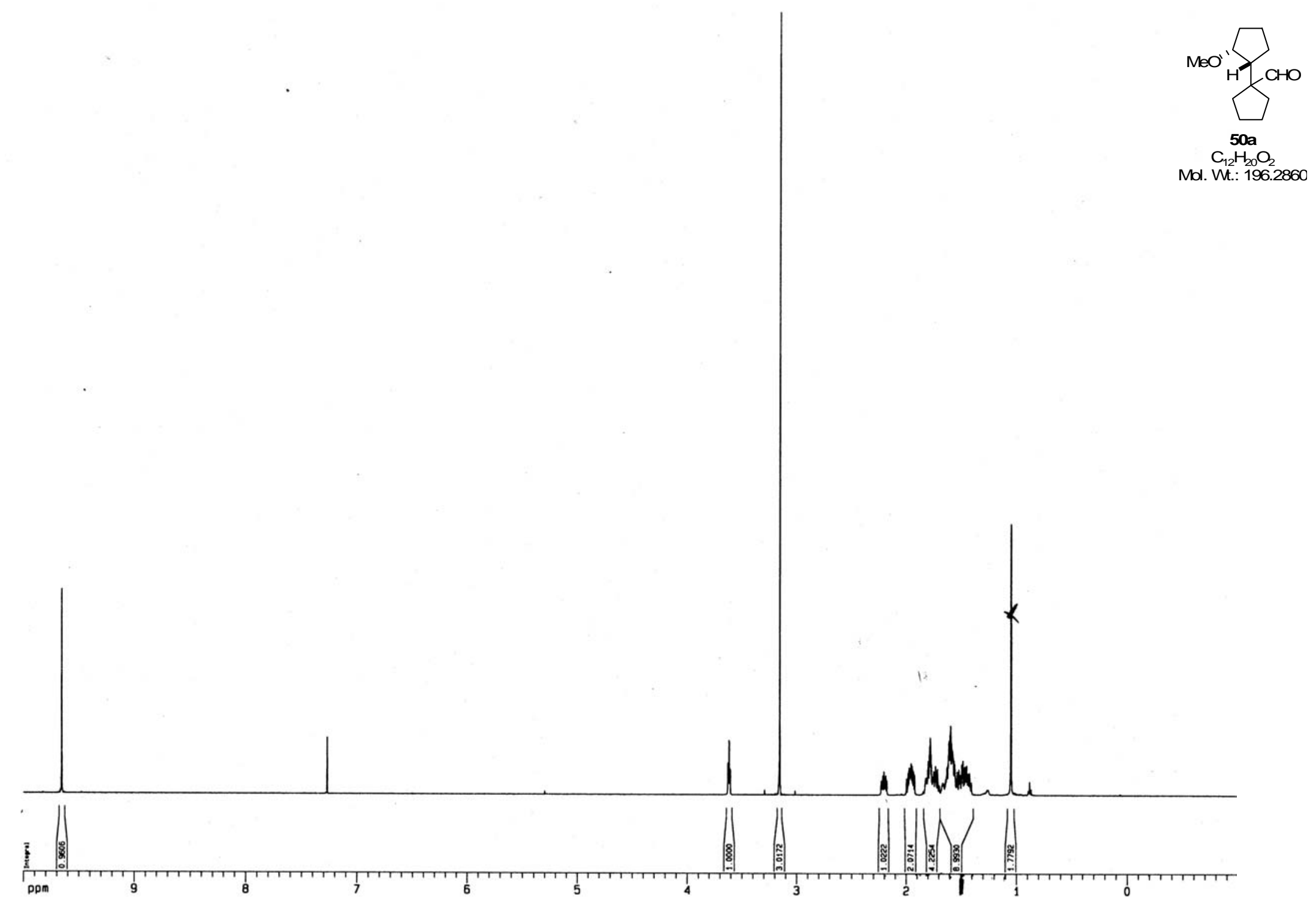




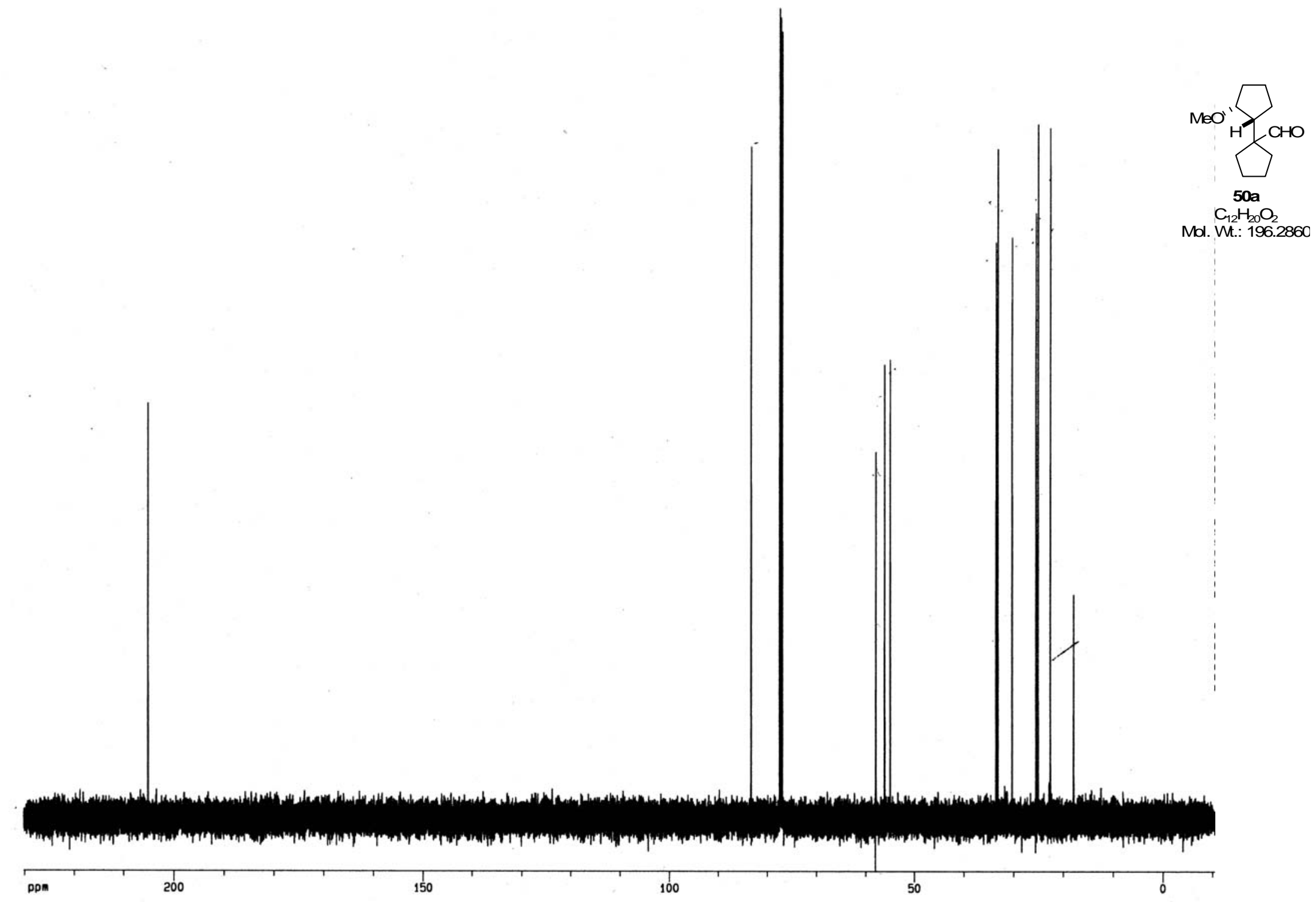




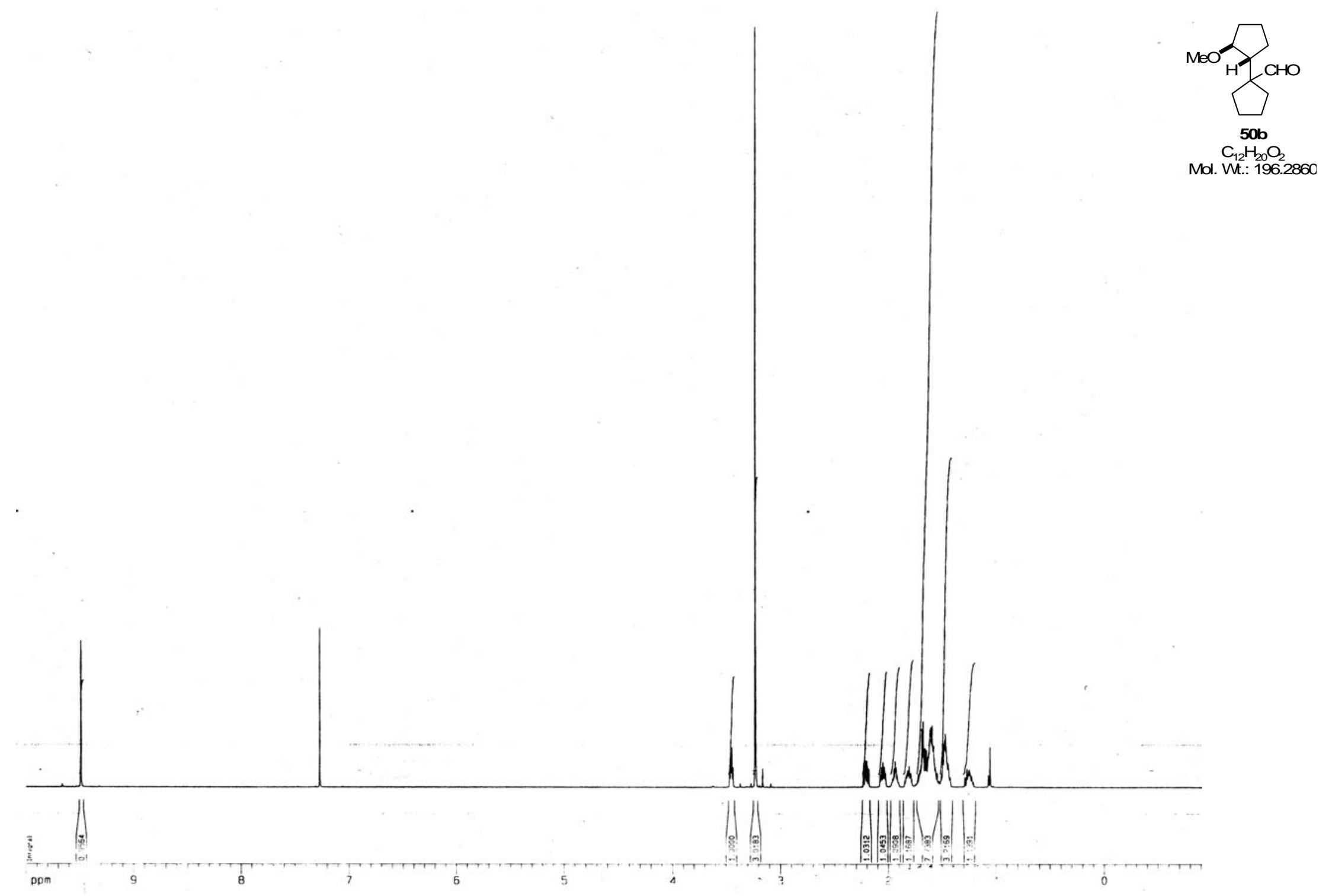




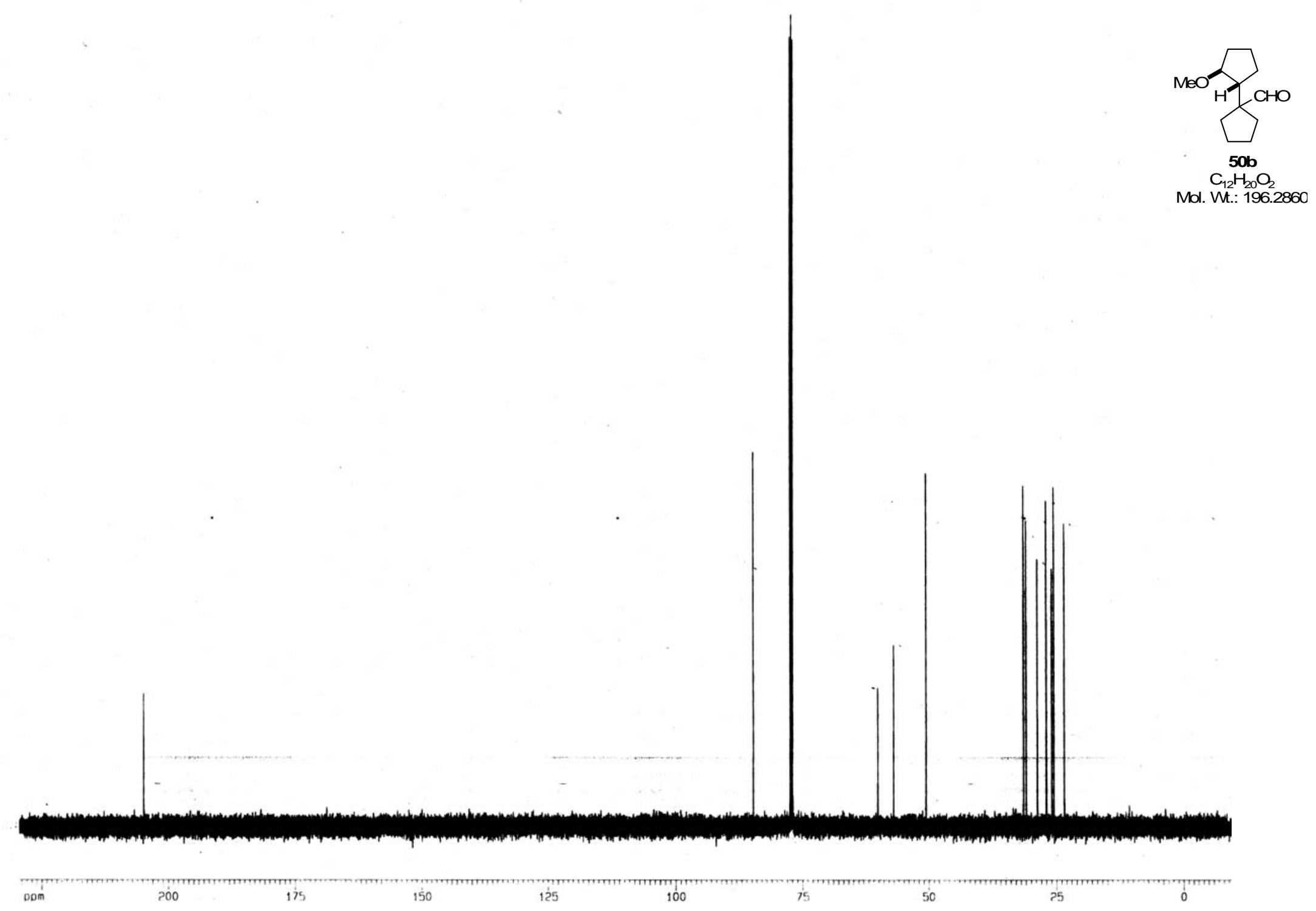




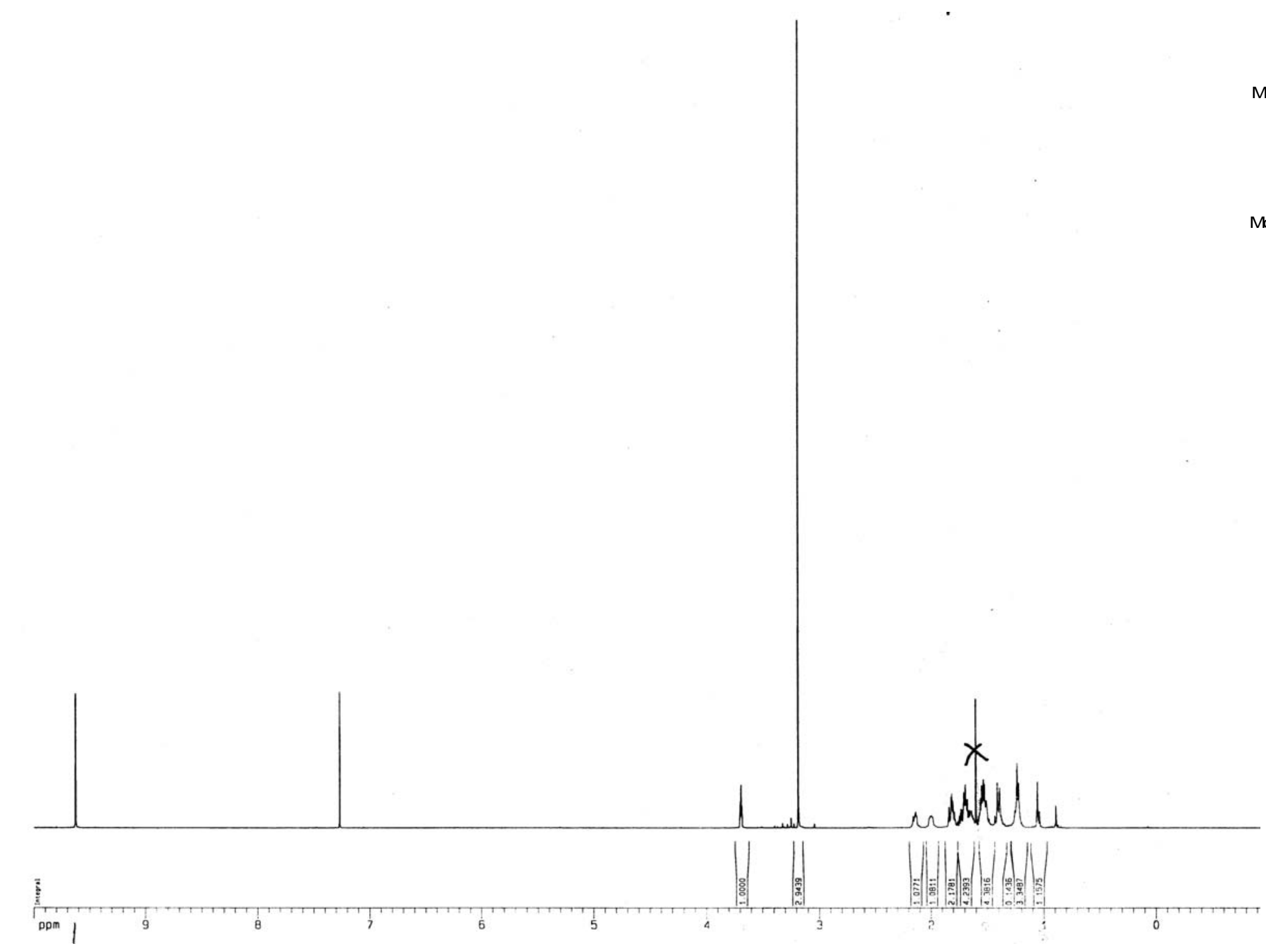




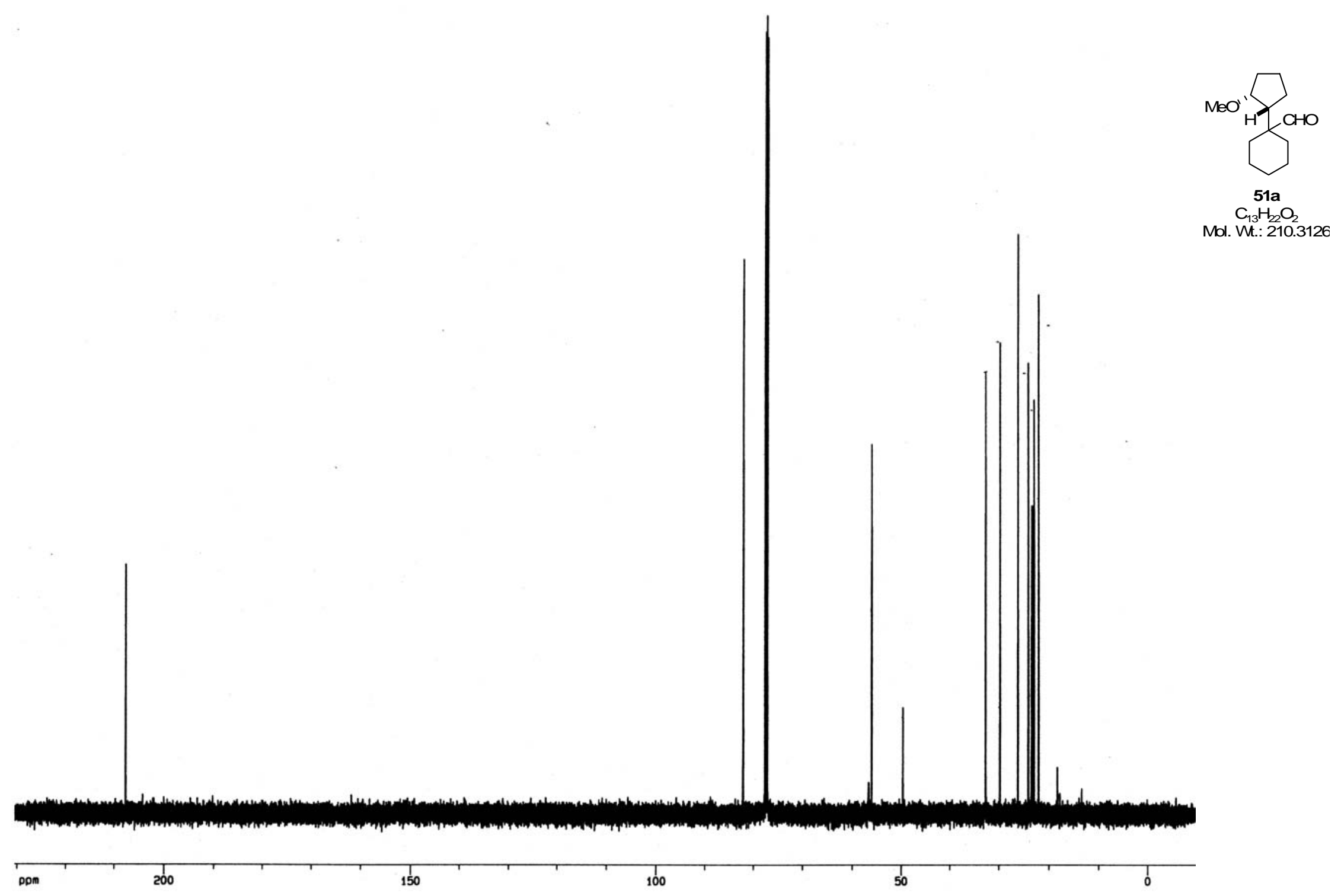




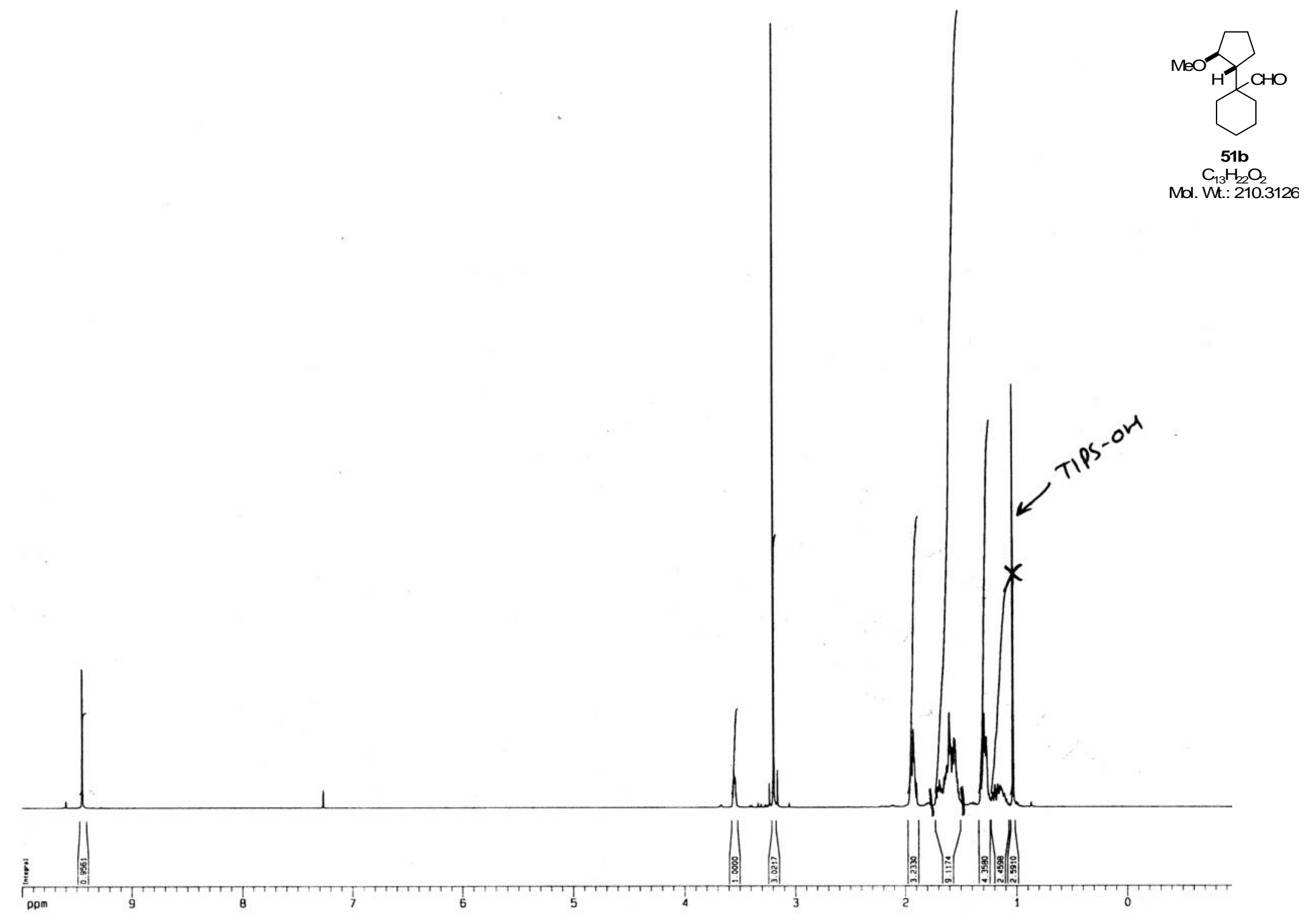




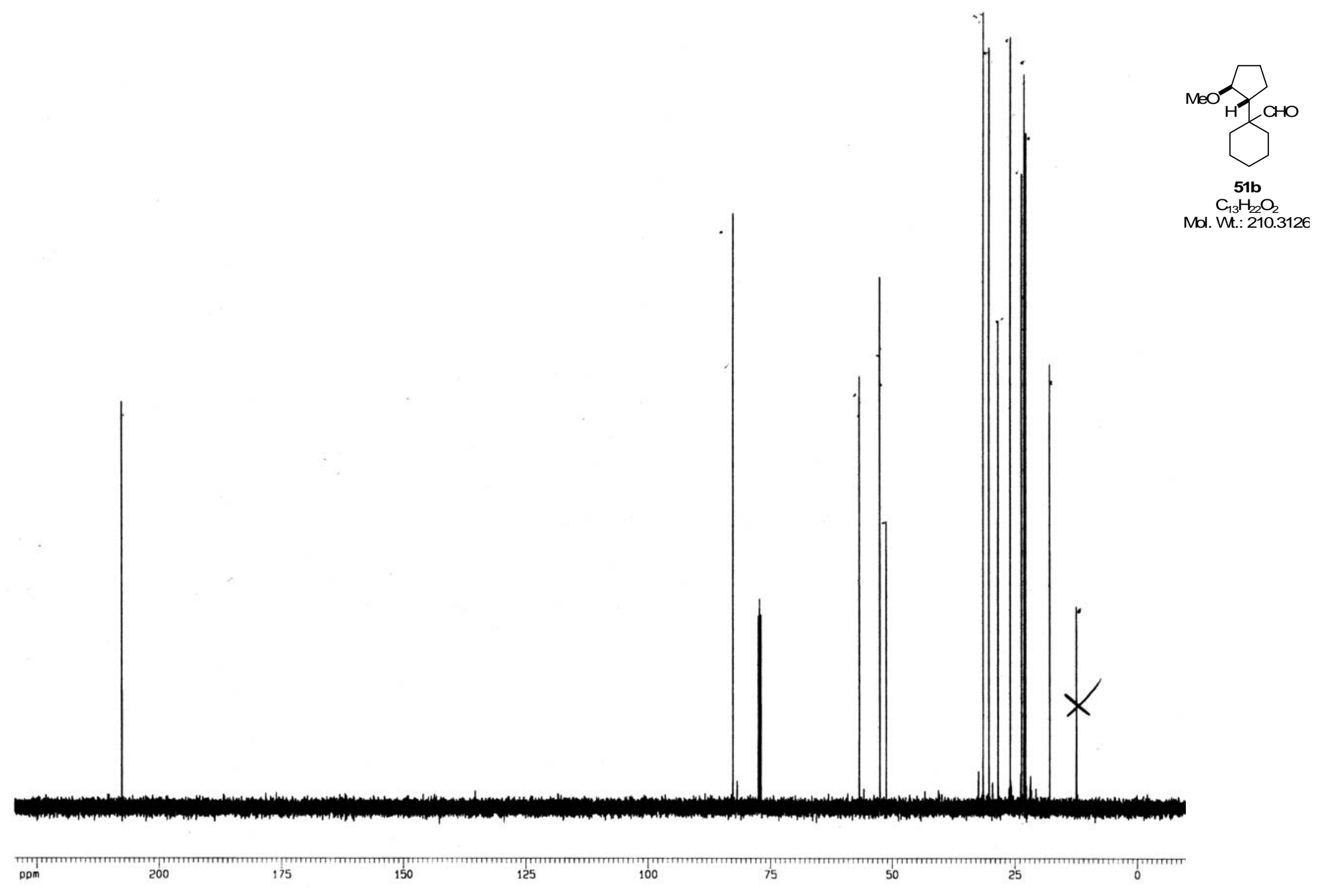




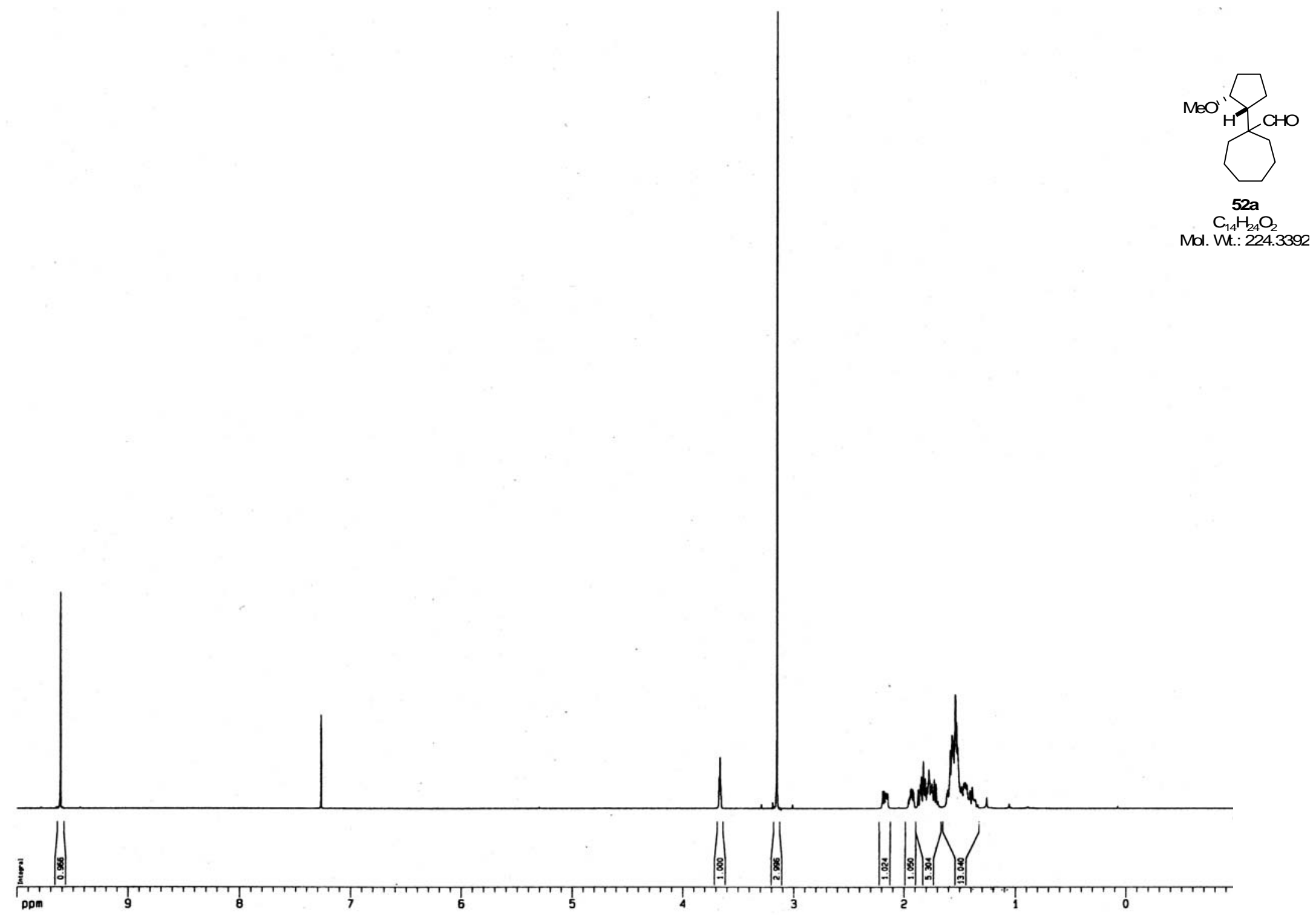




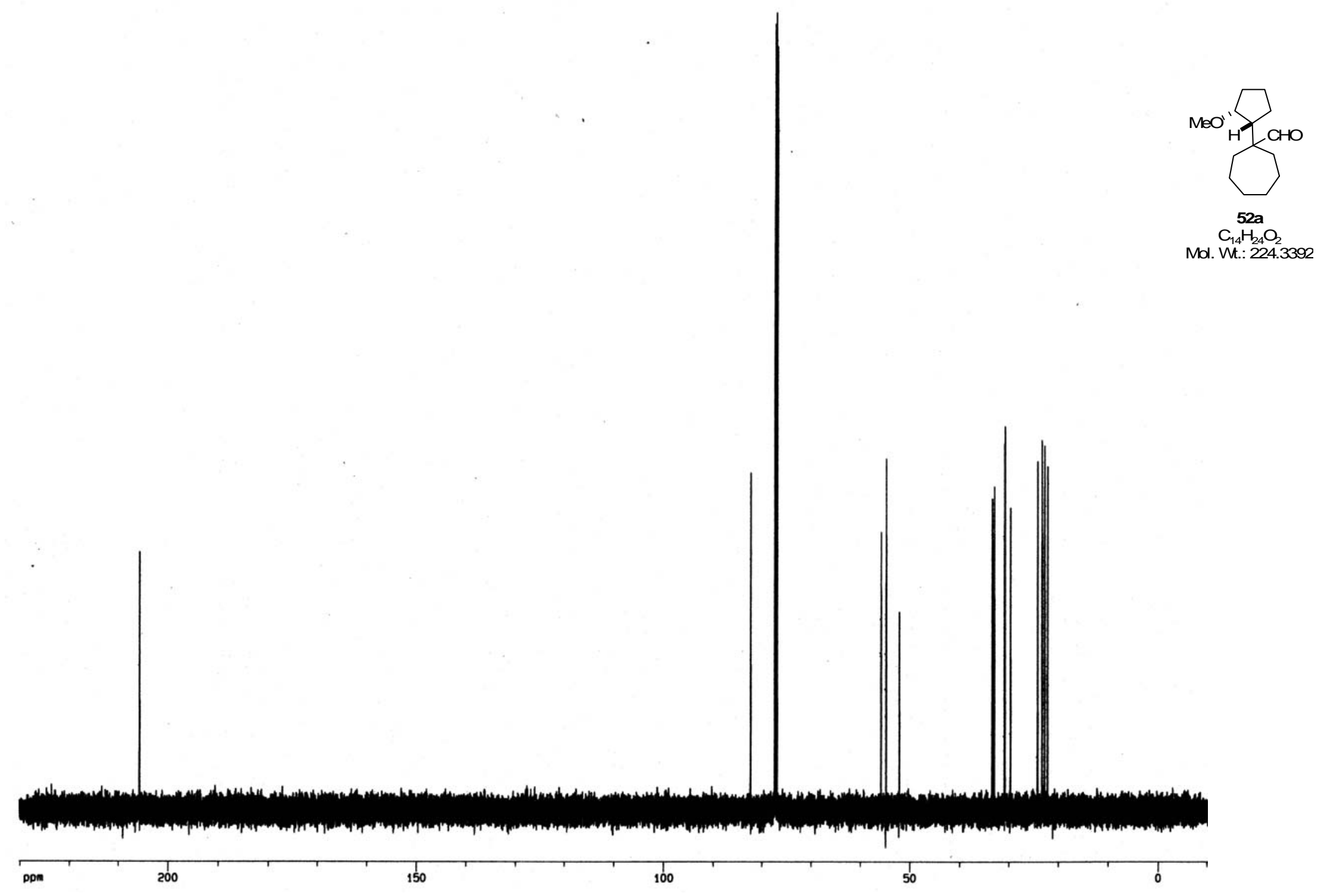



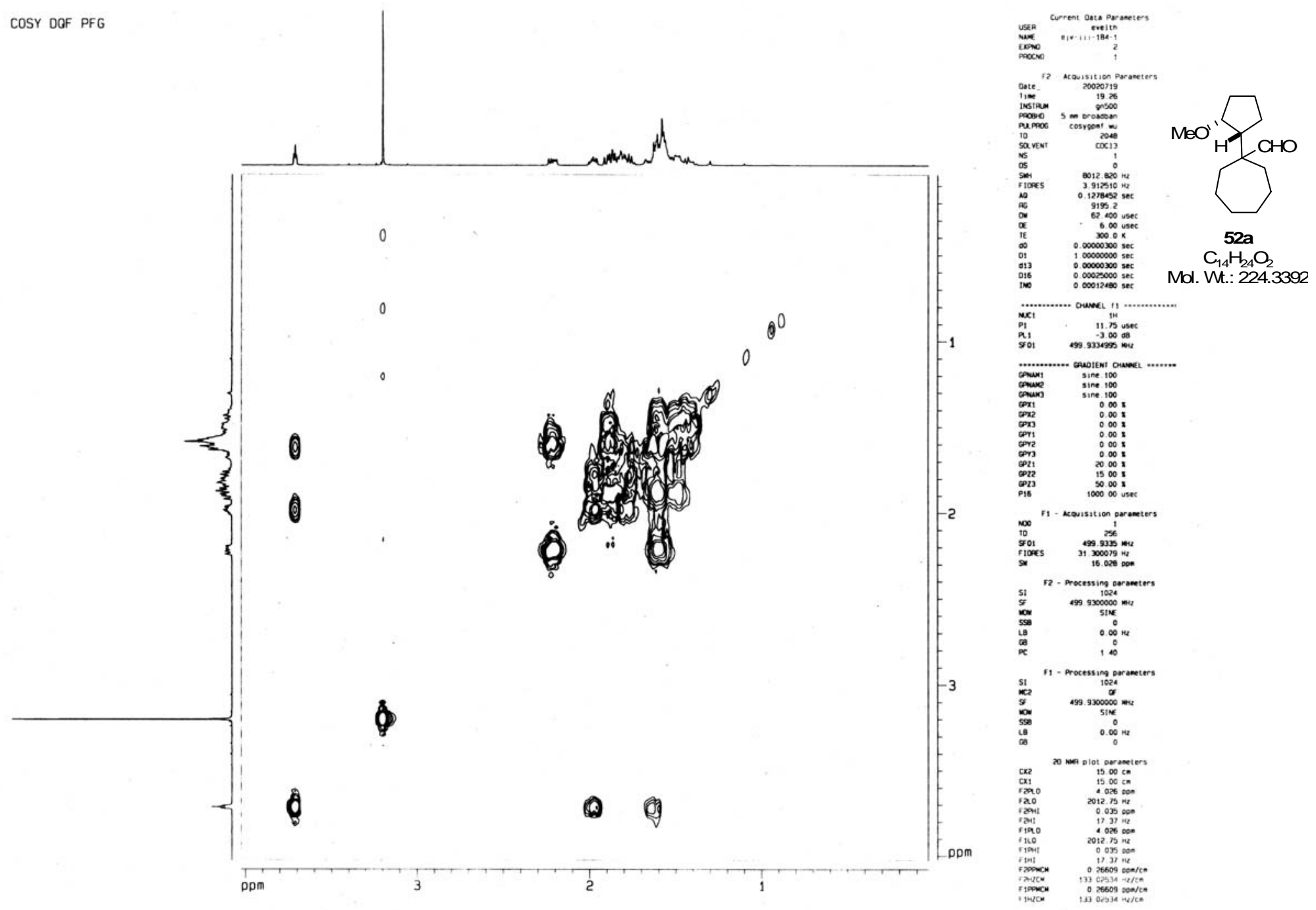


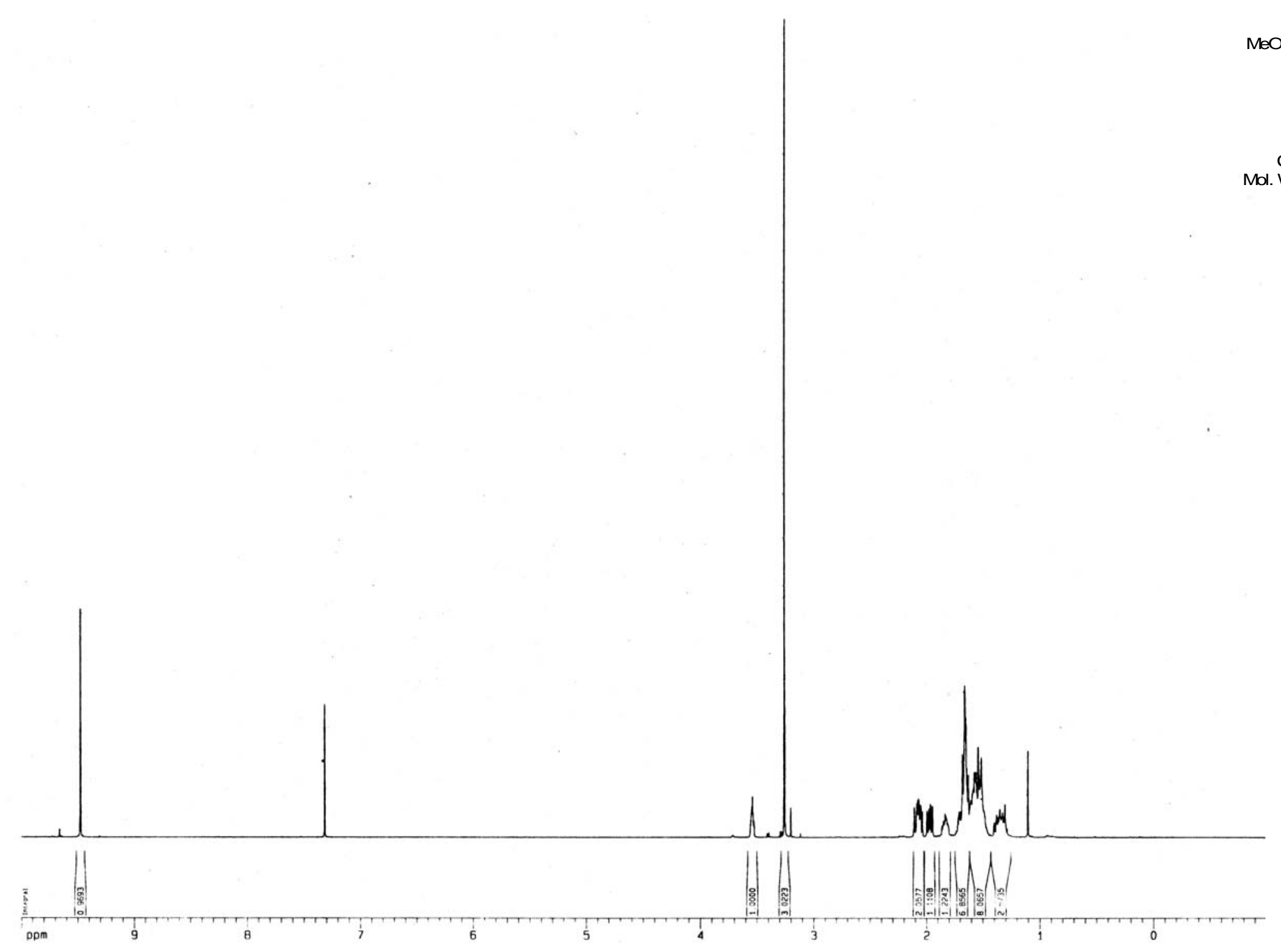




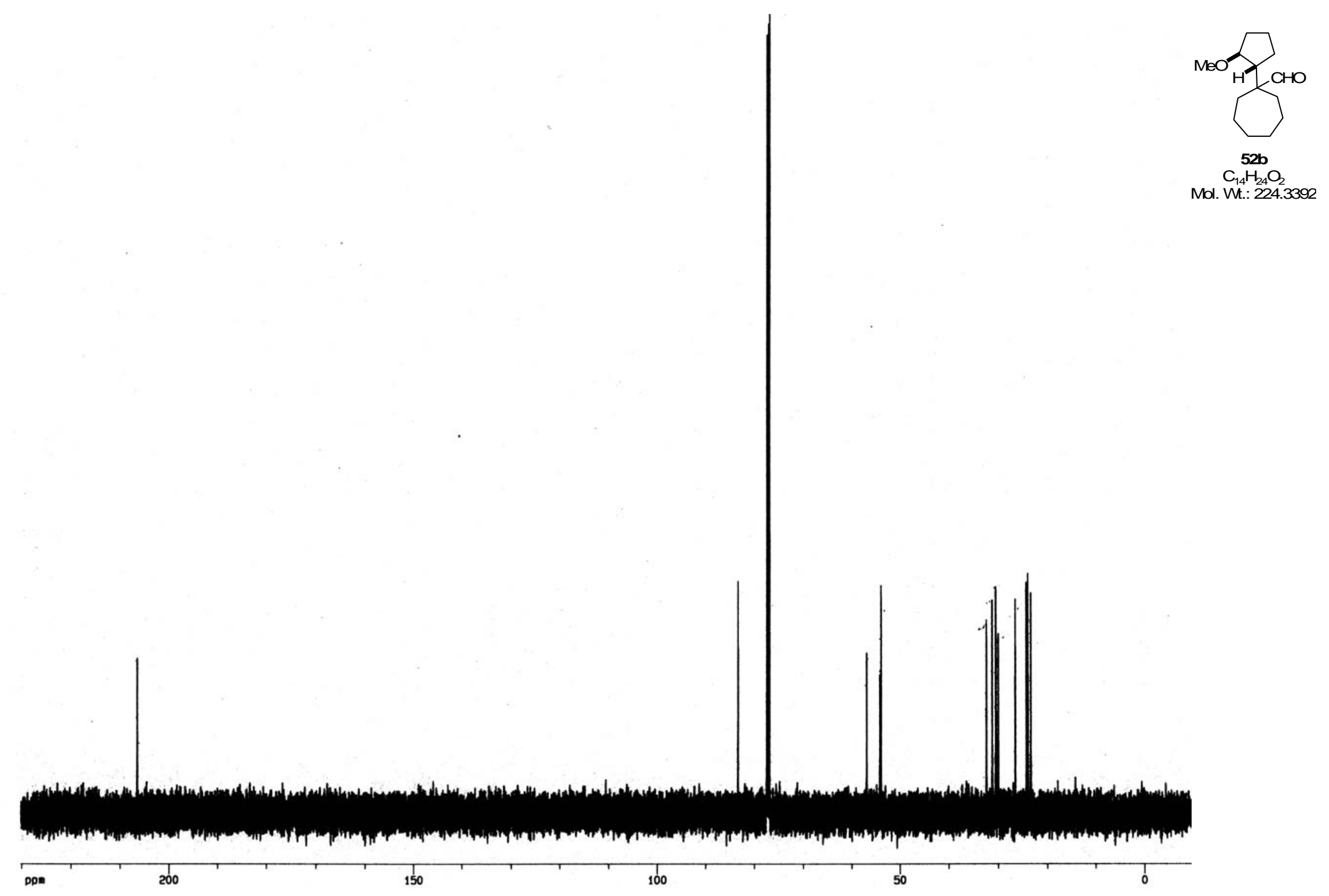



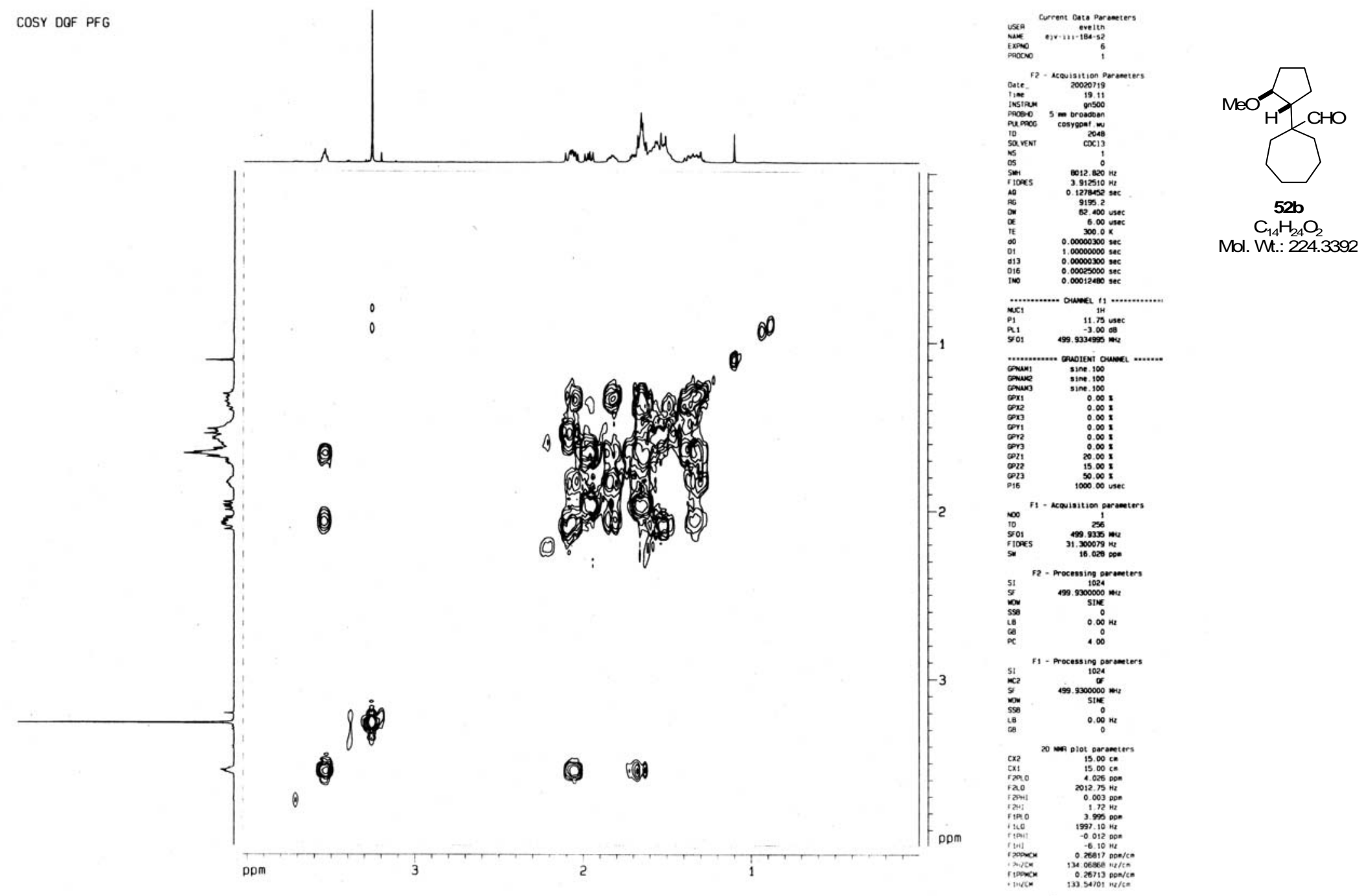

Mol. Wt.: 224.3392 


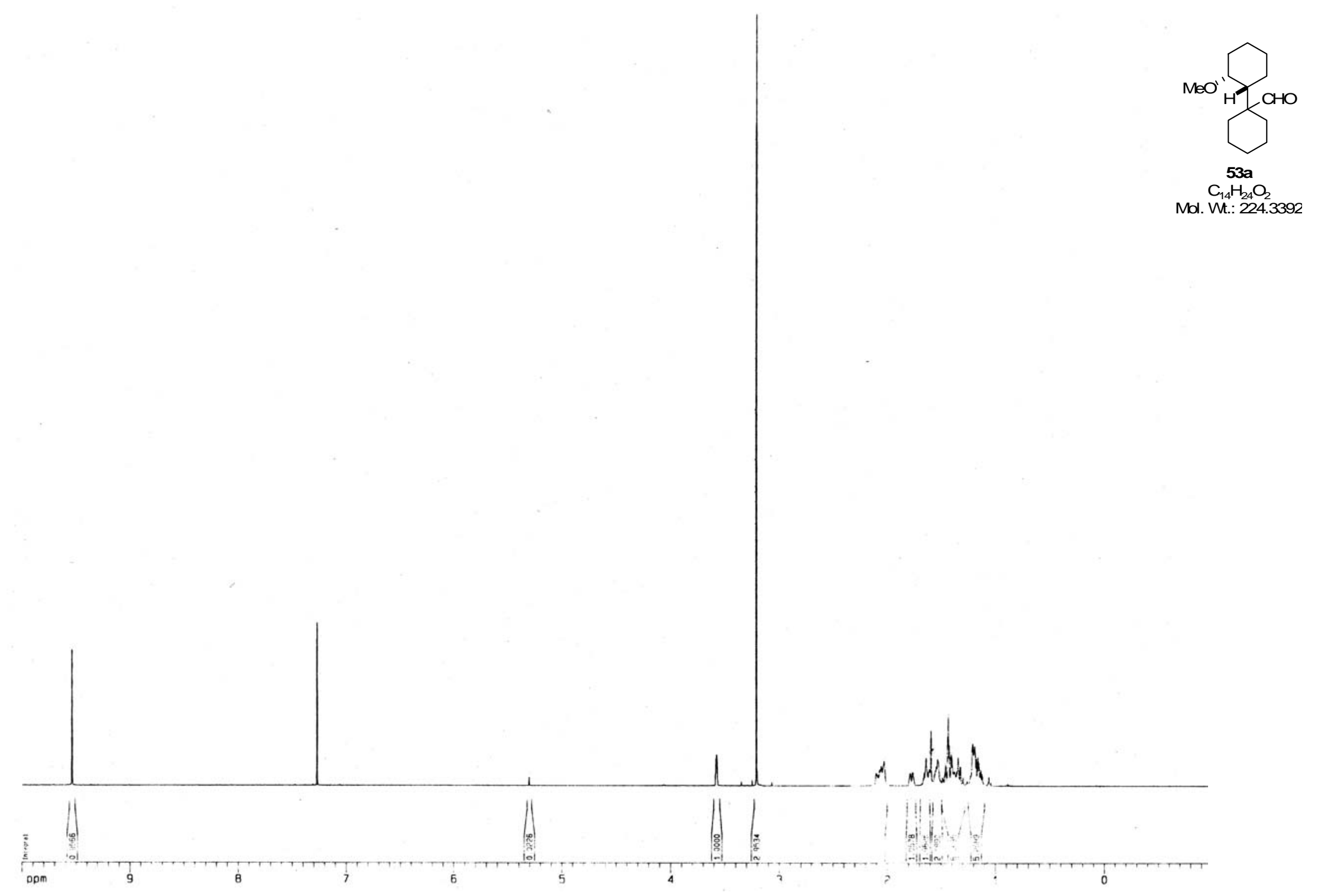




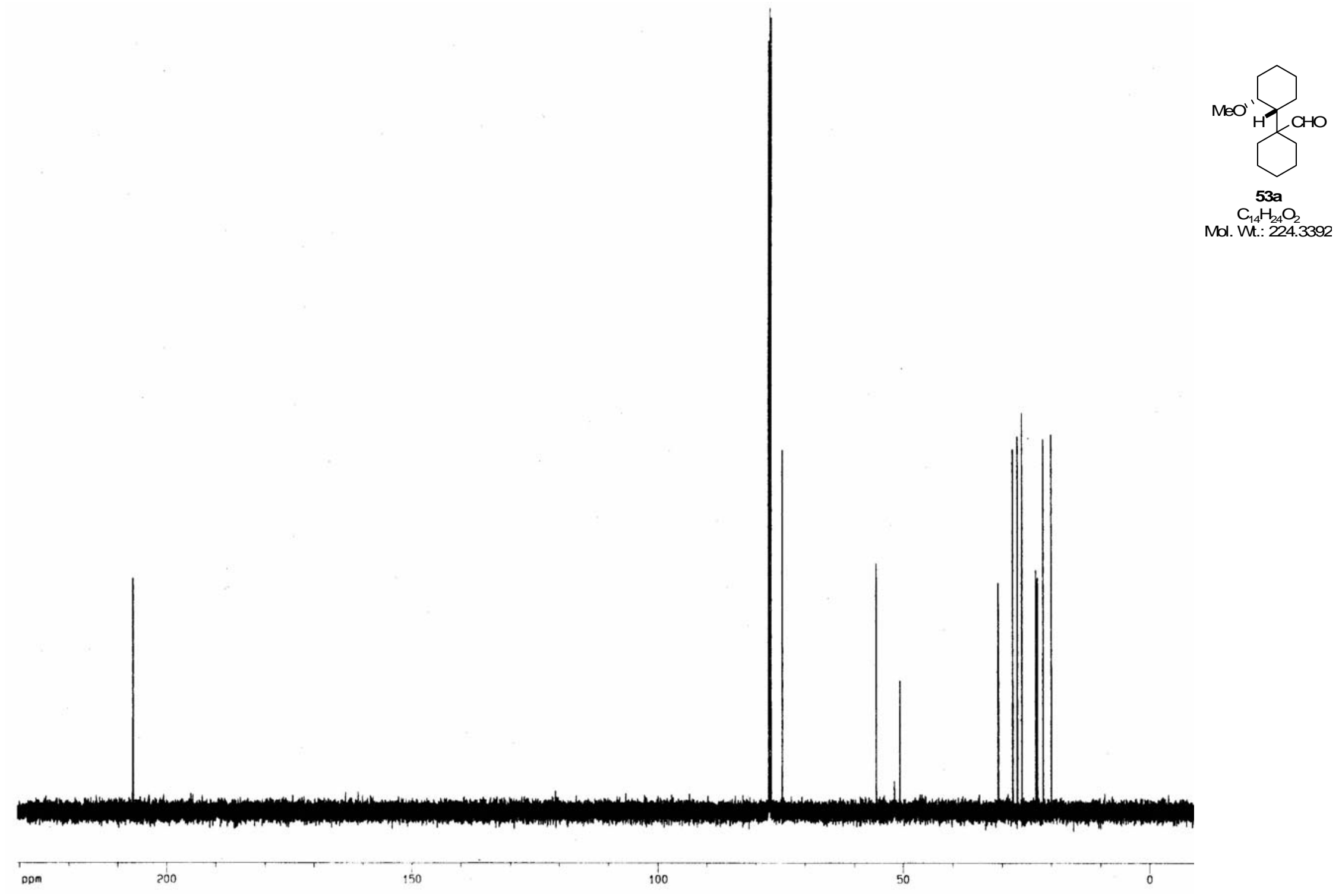




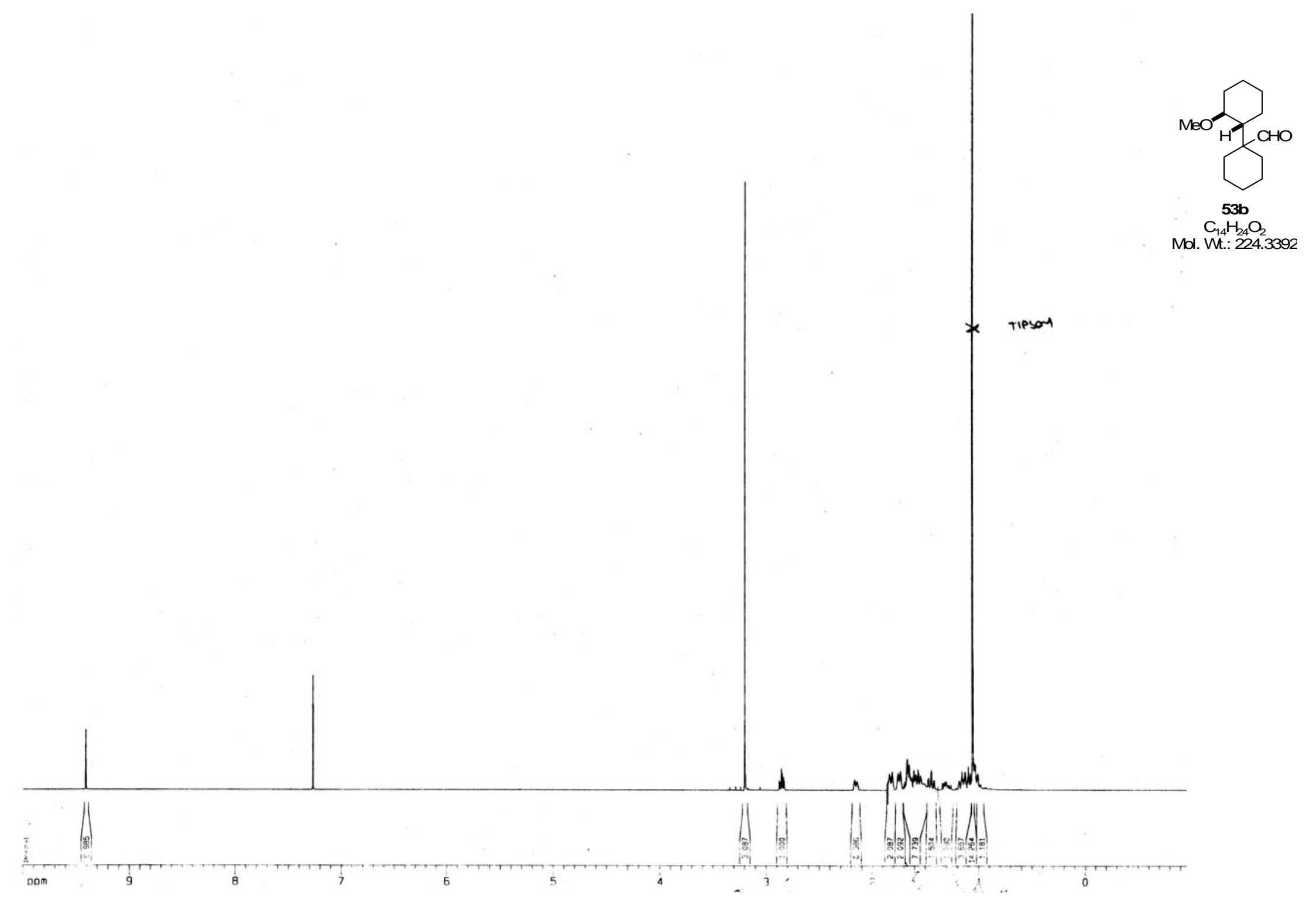




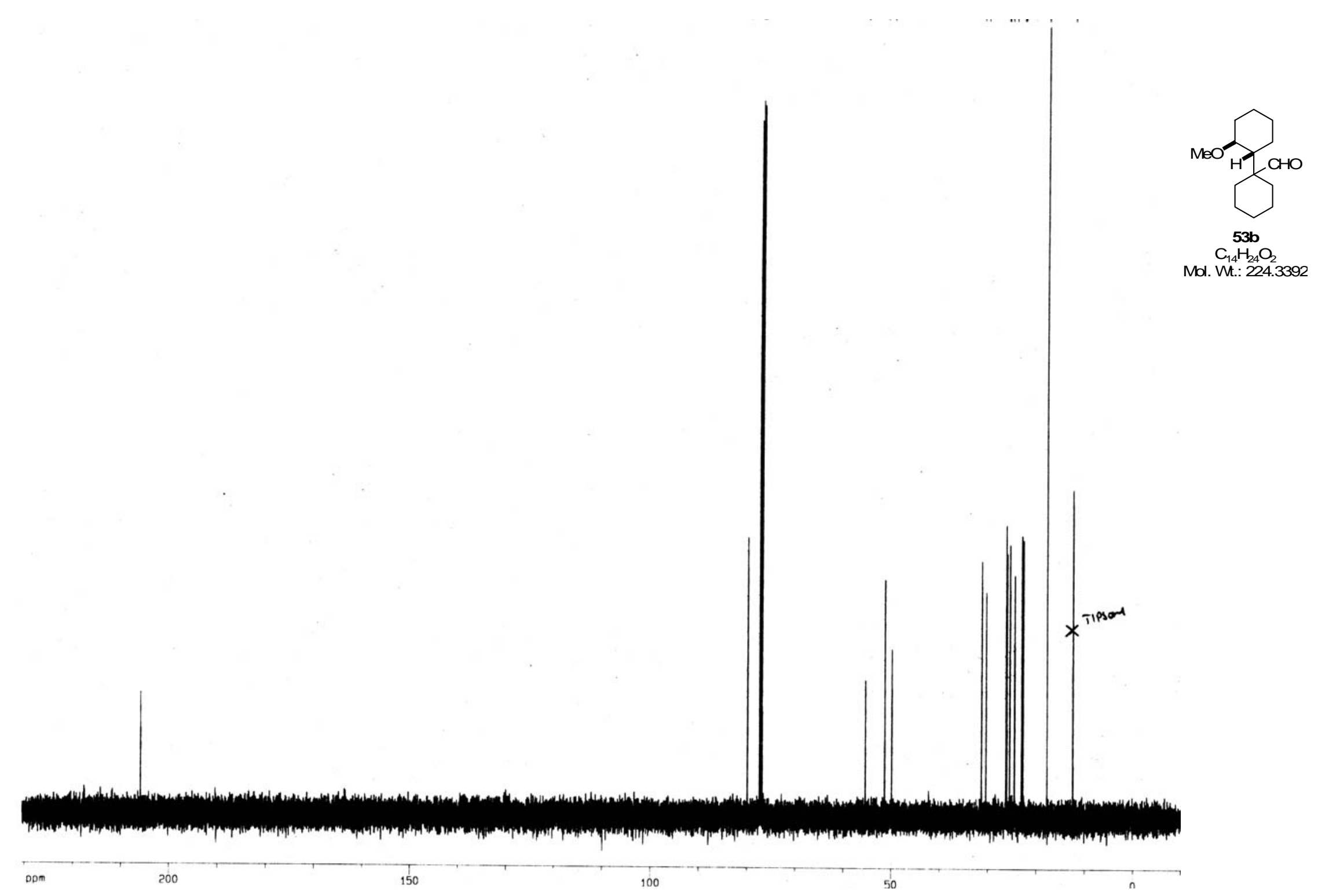




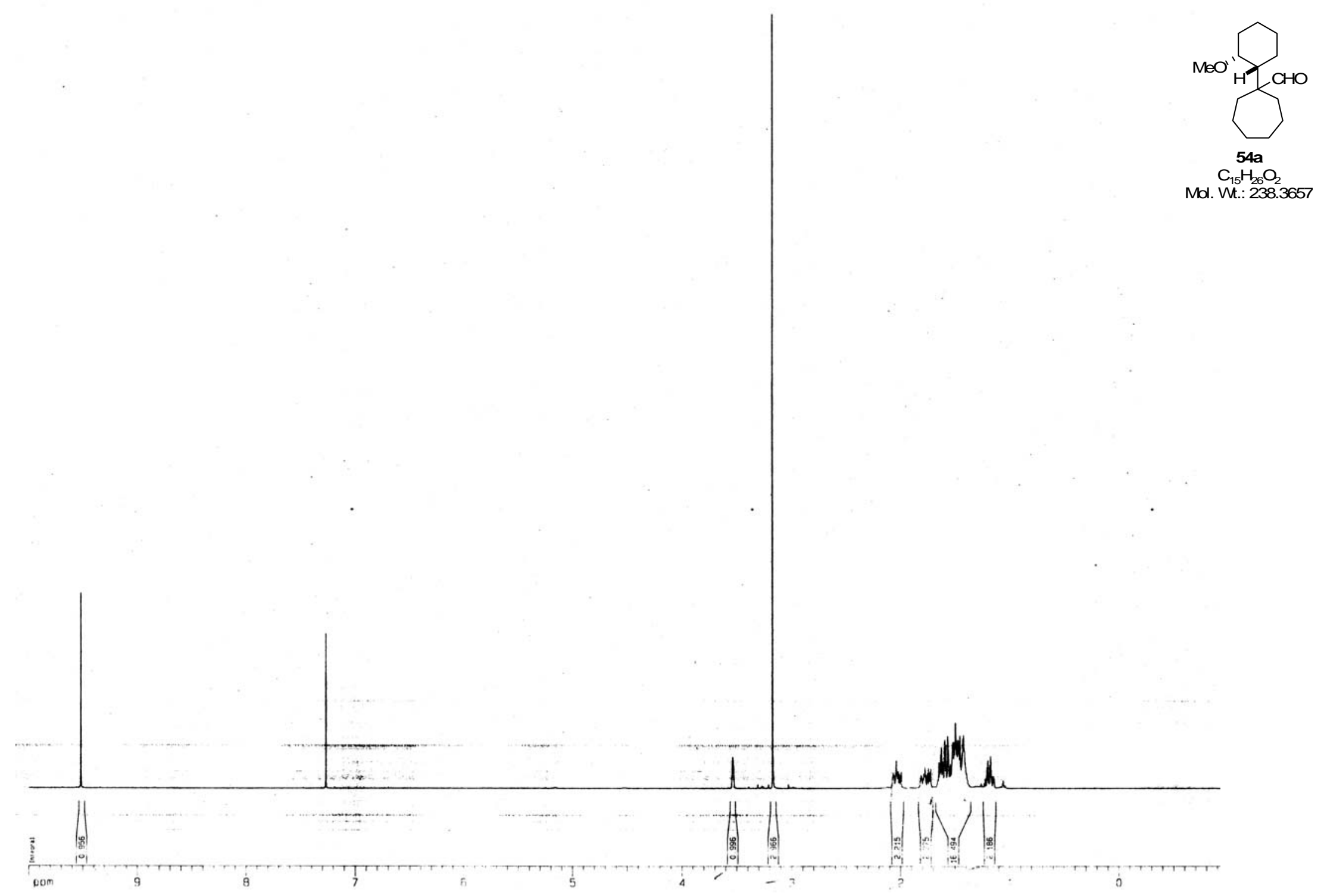




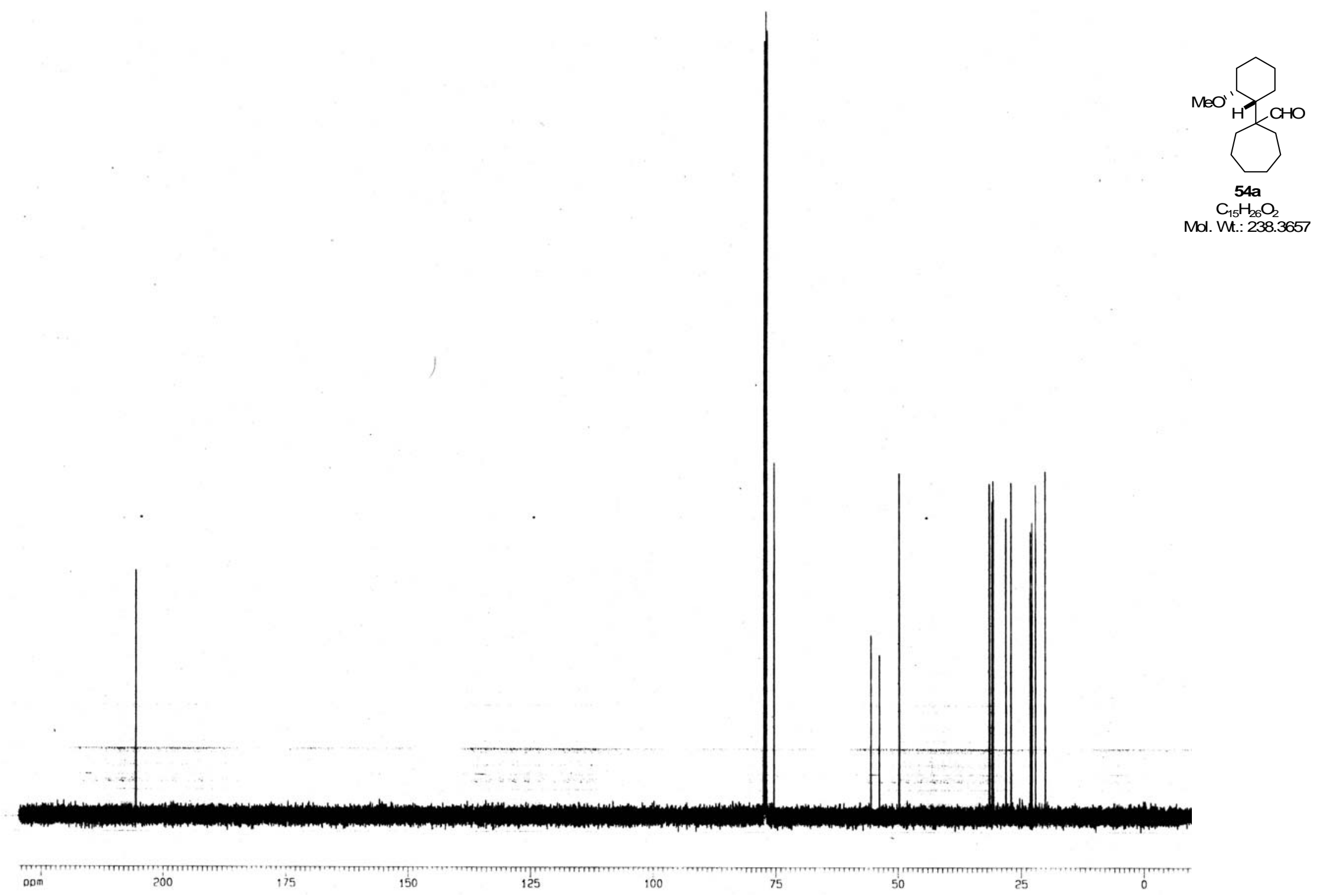




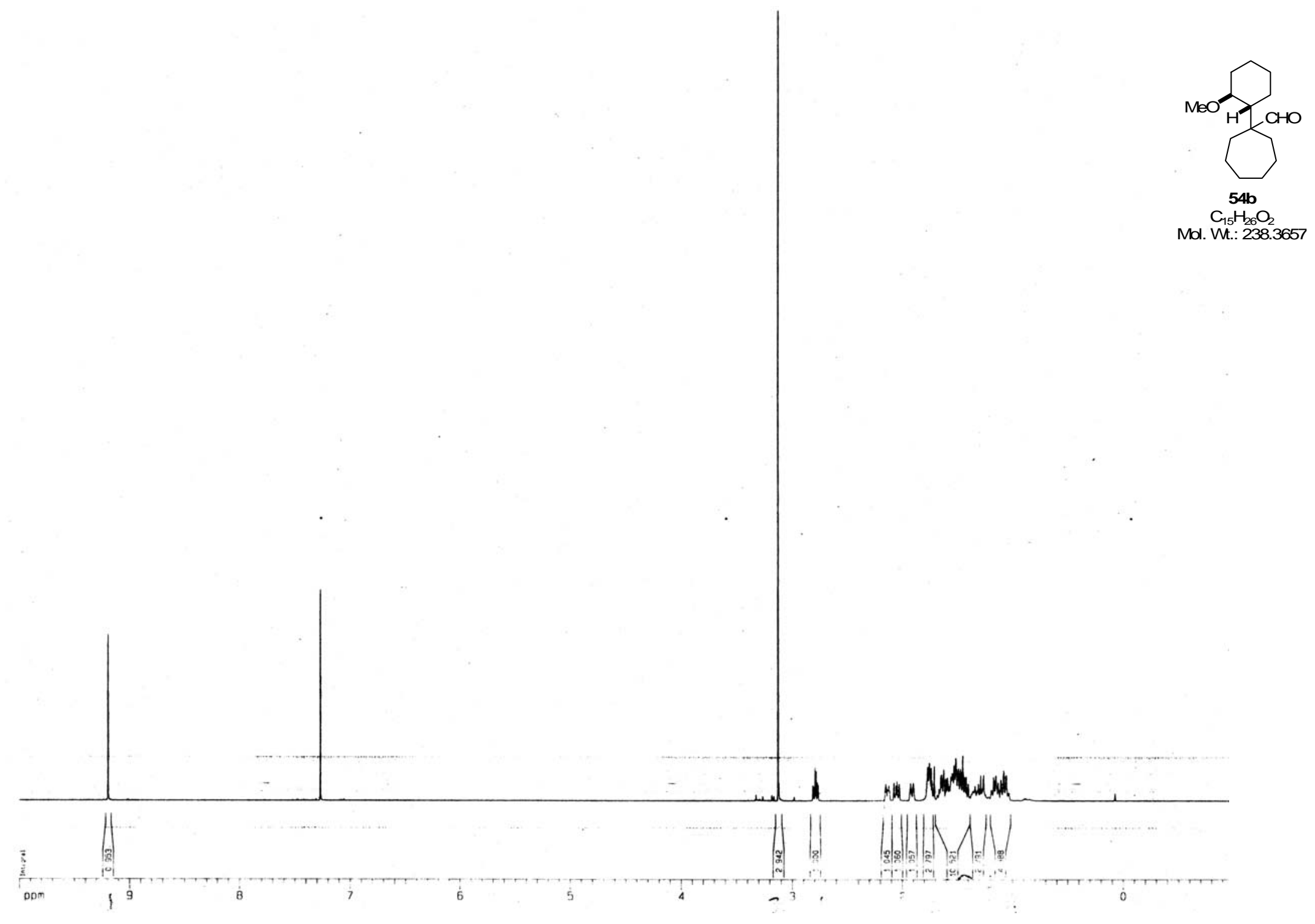




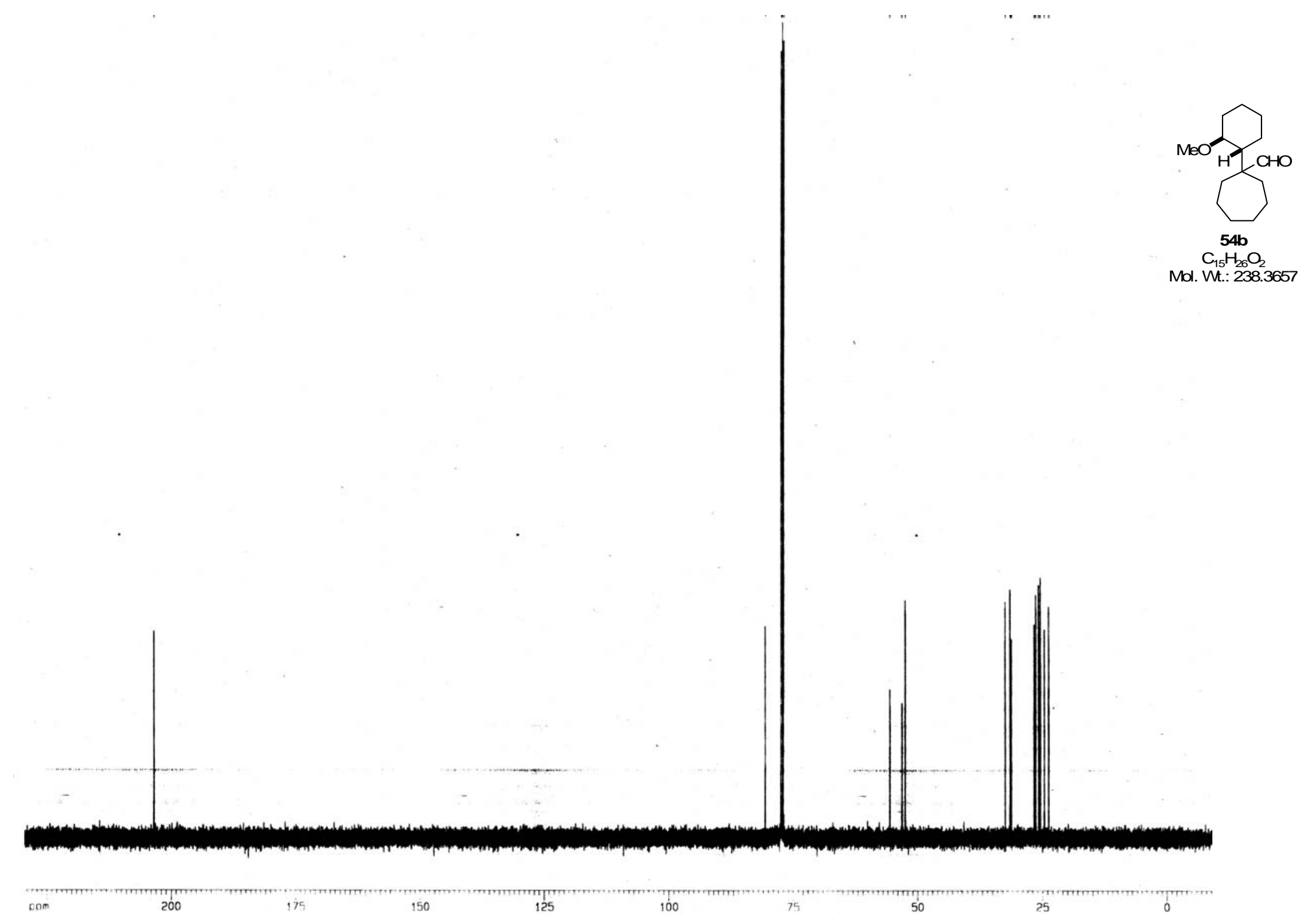




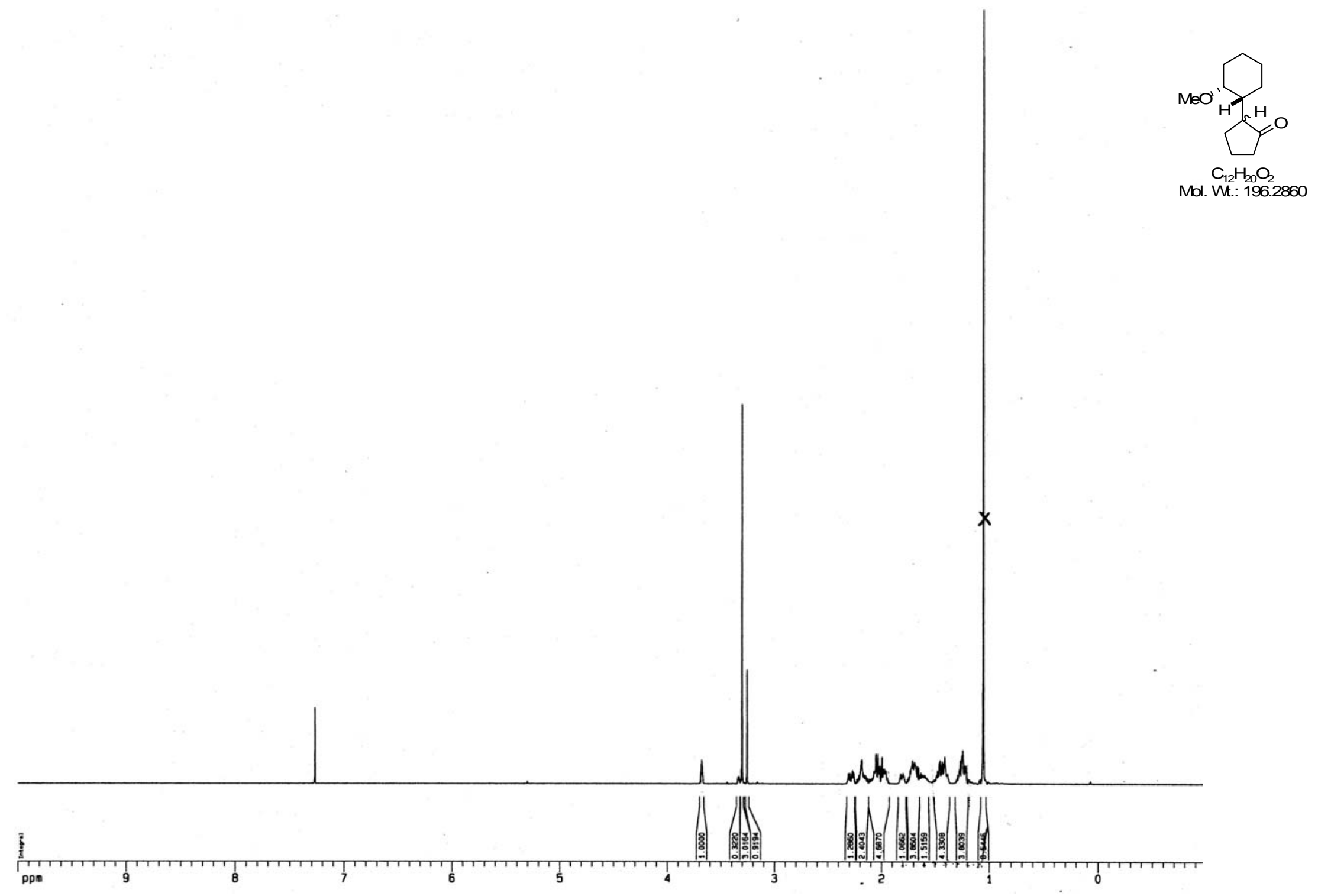




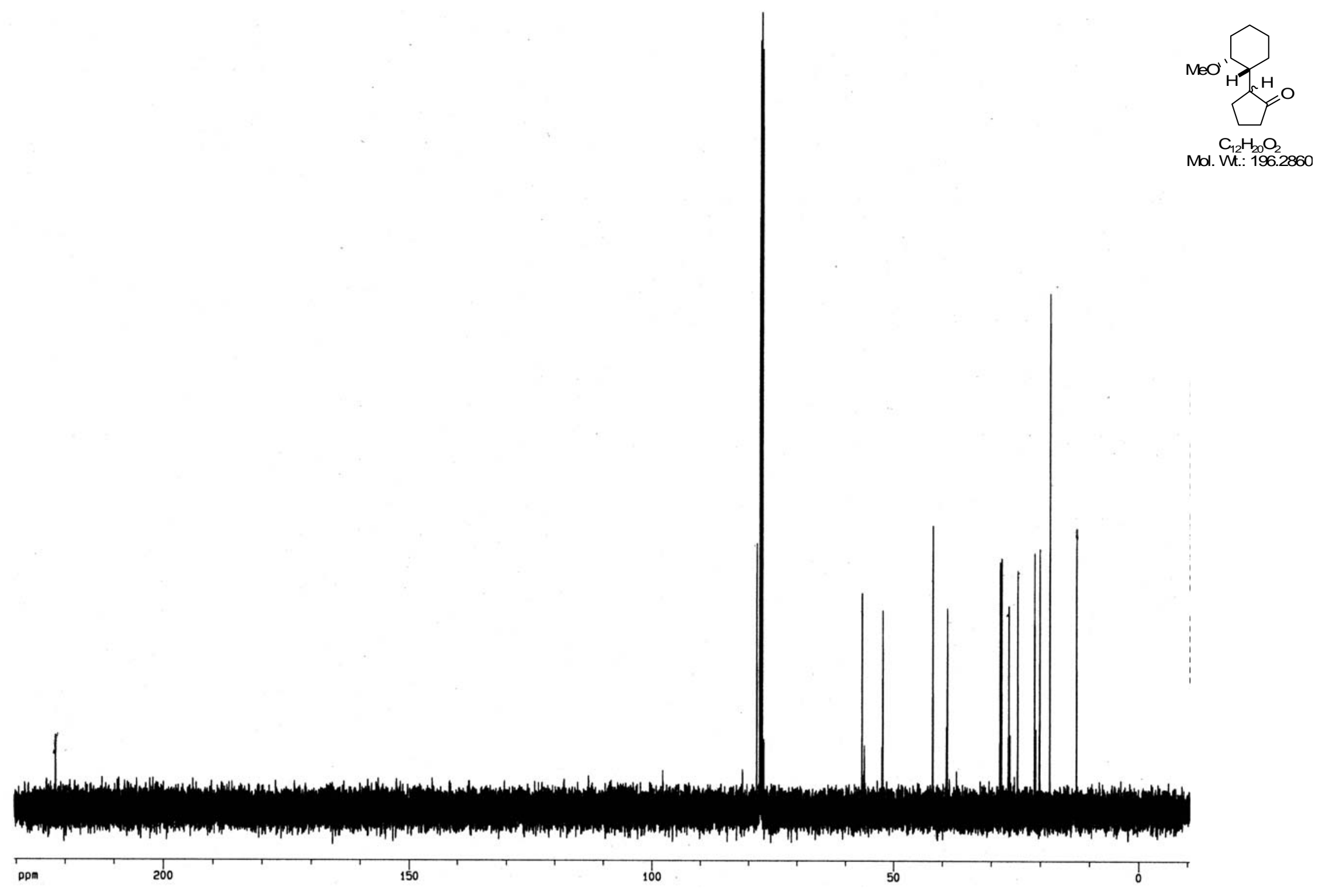


Overman and Velthuisen

S

96

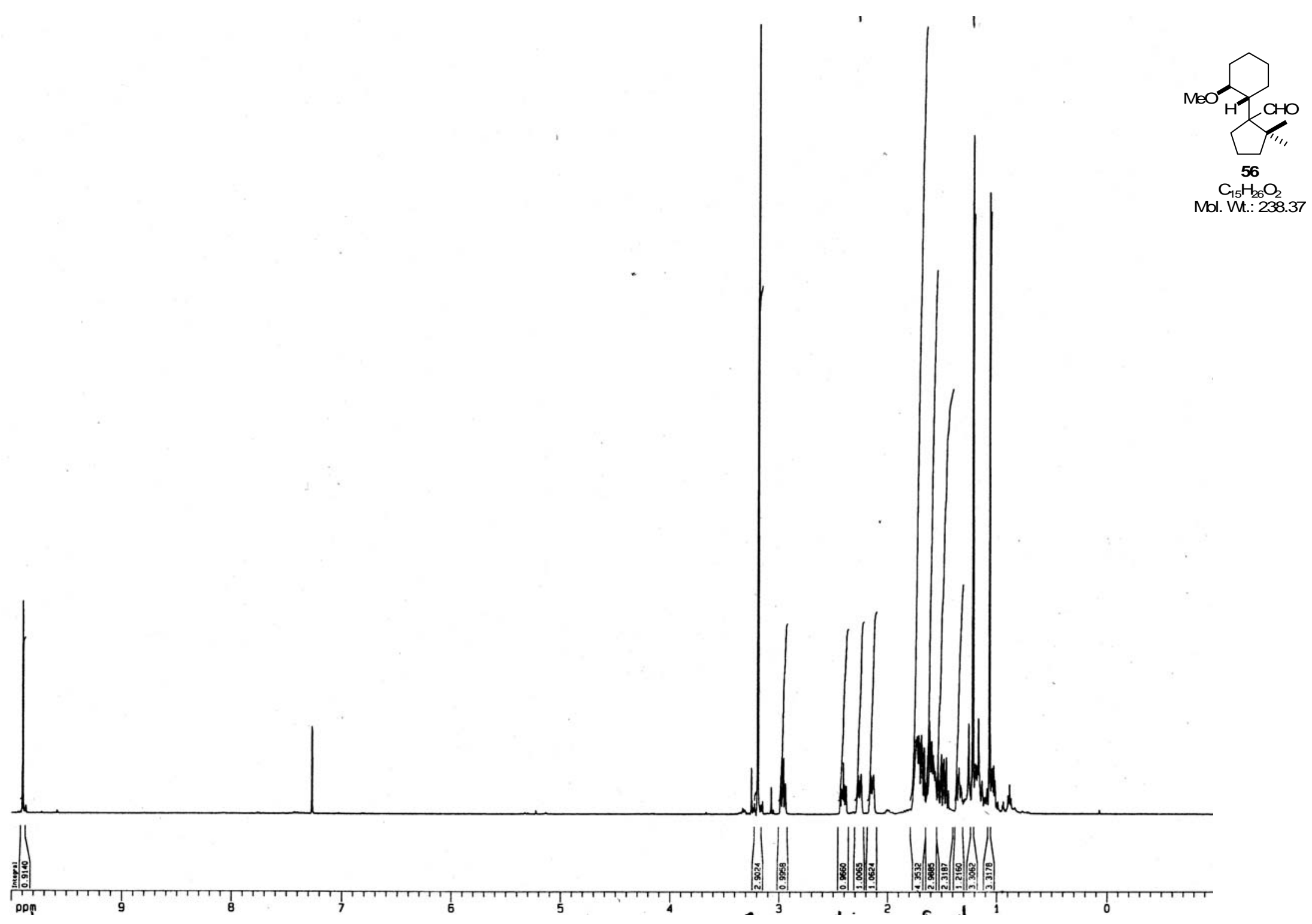




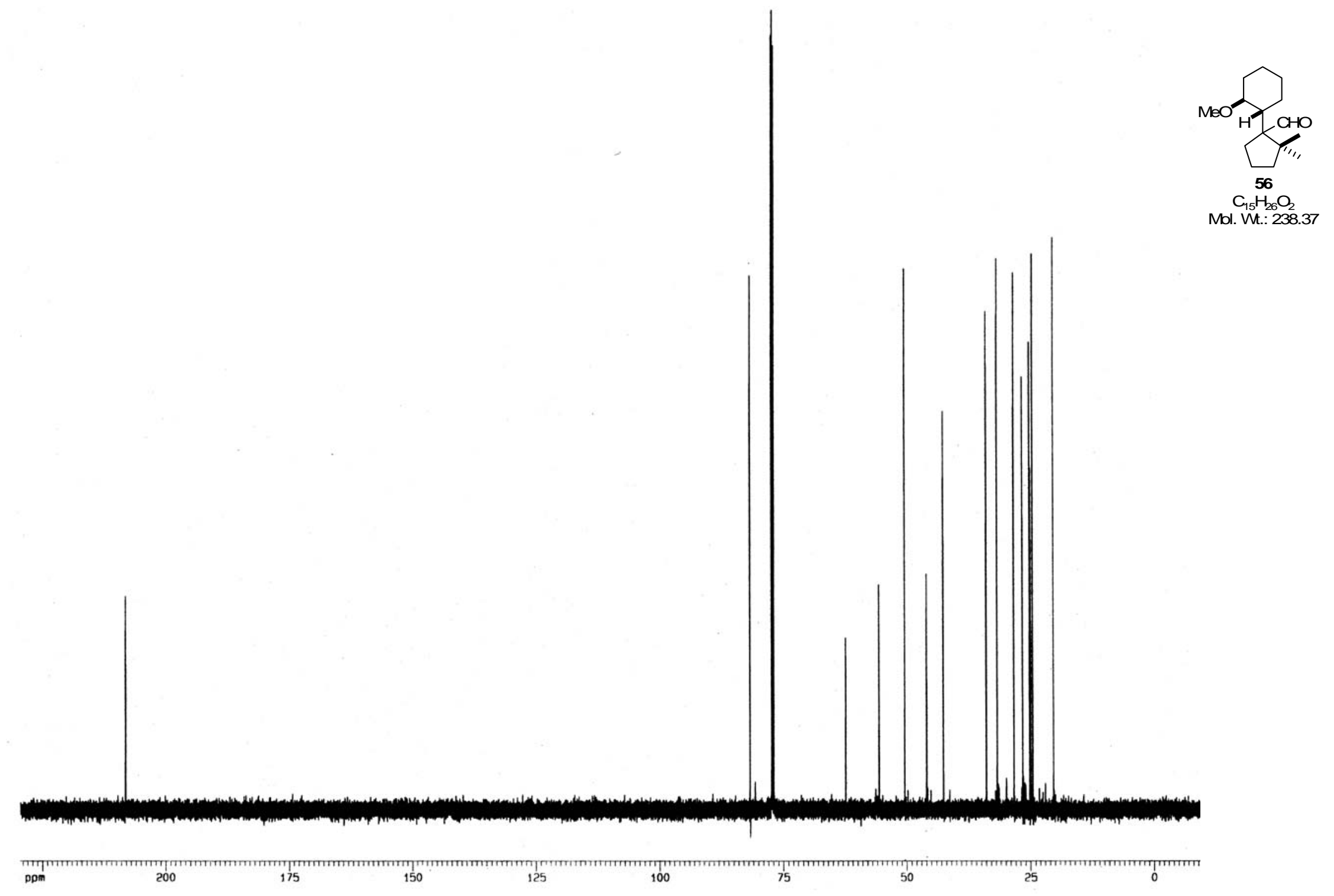




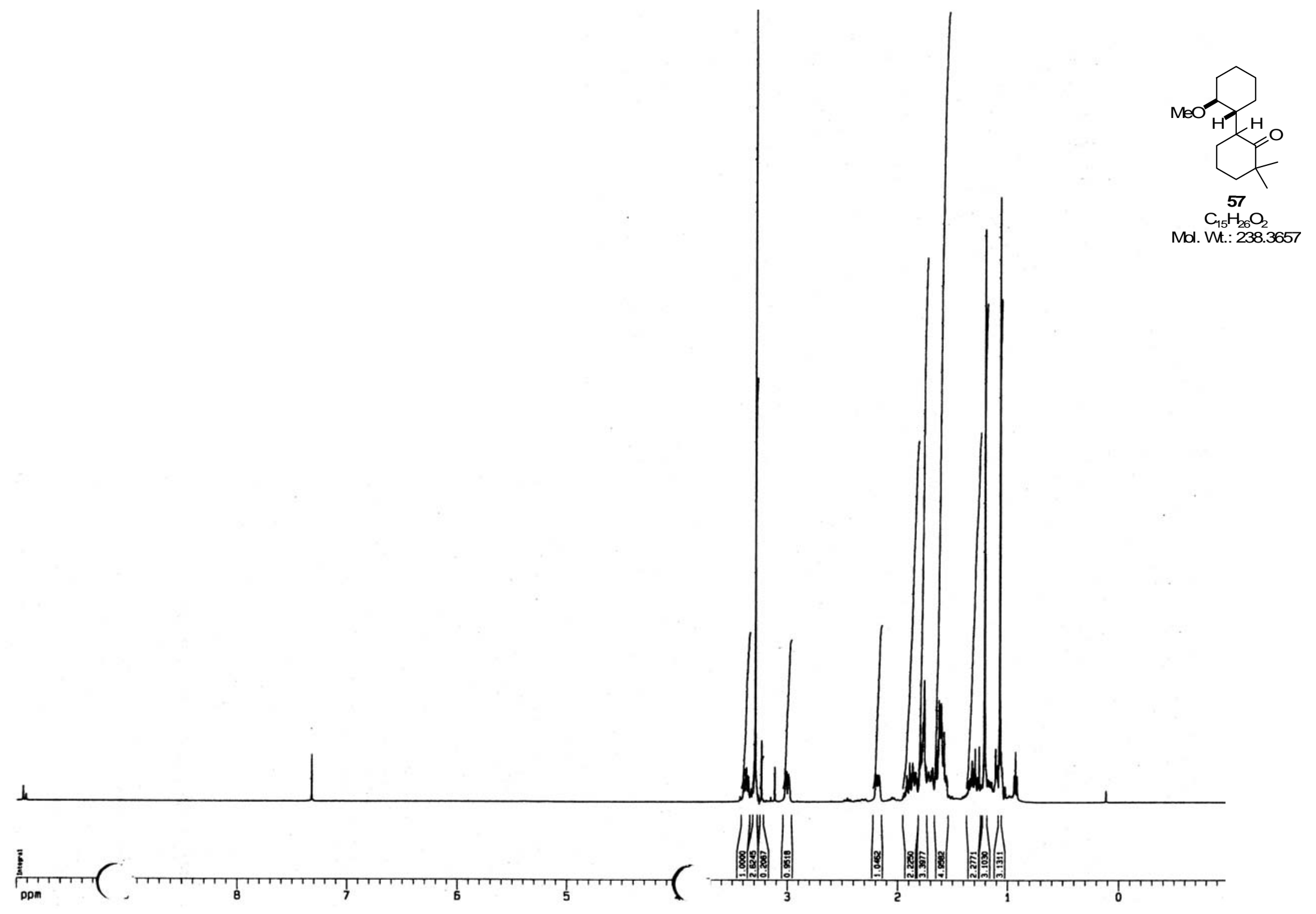




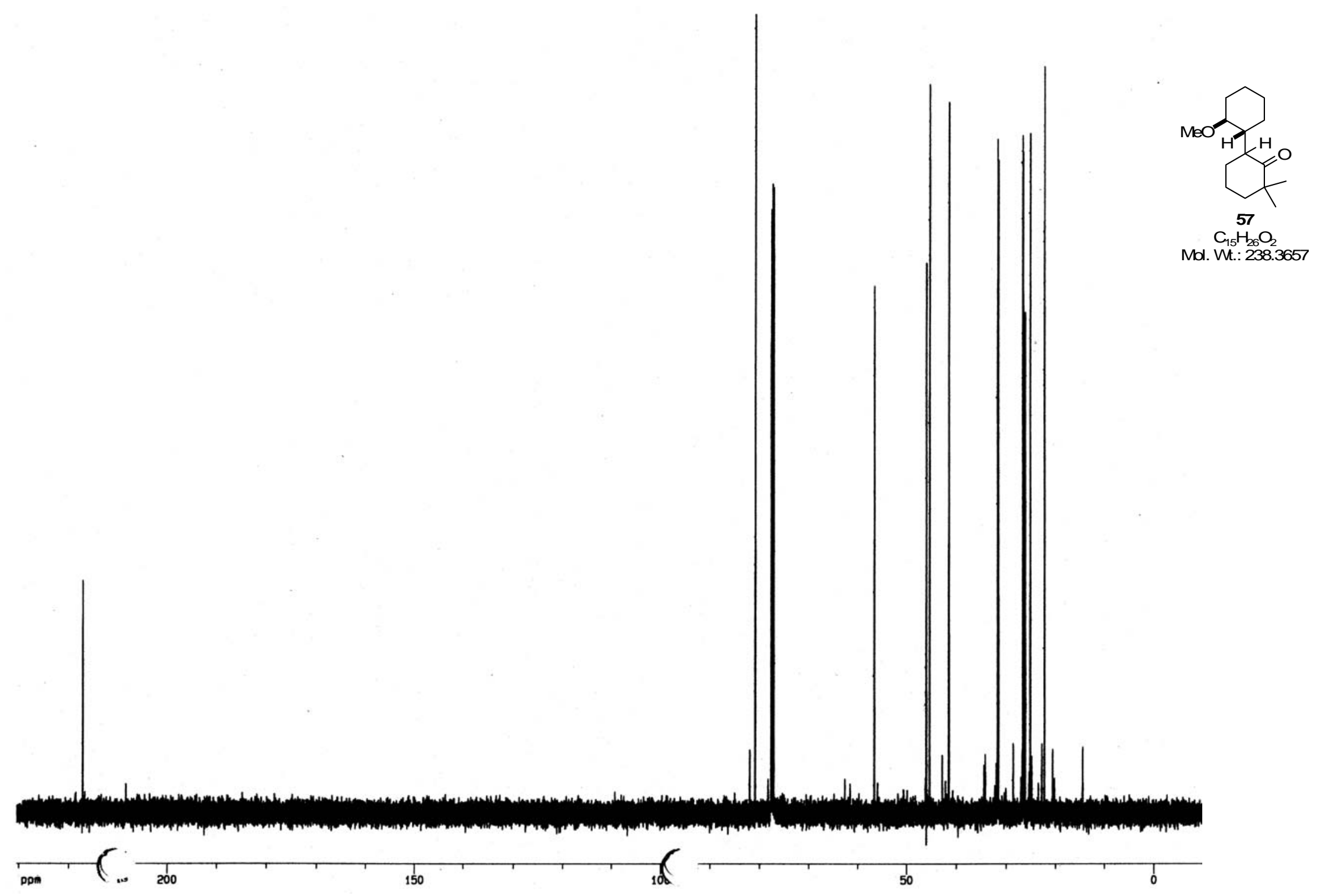




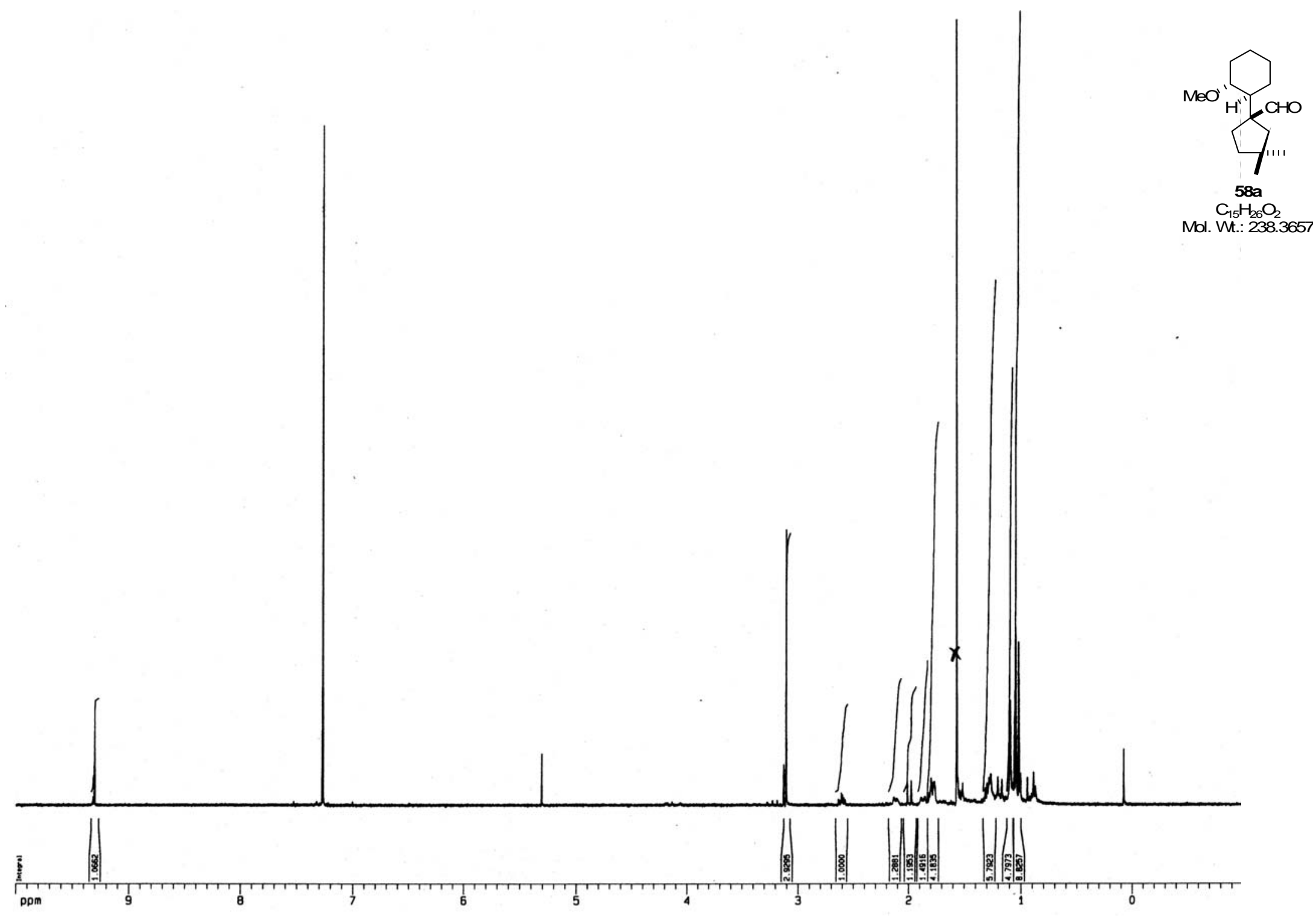




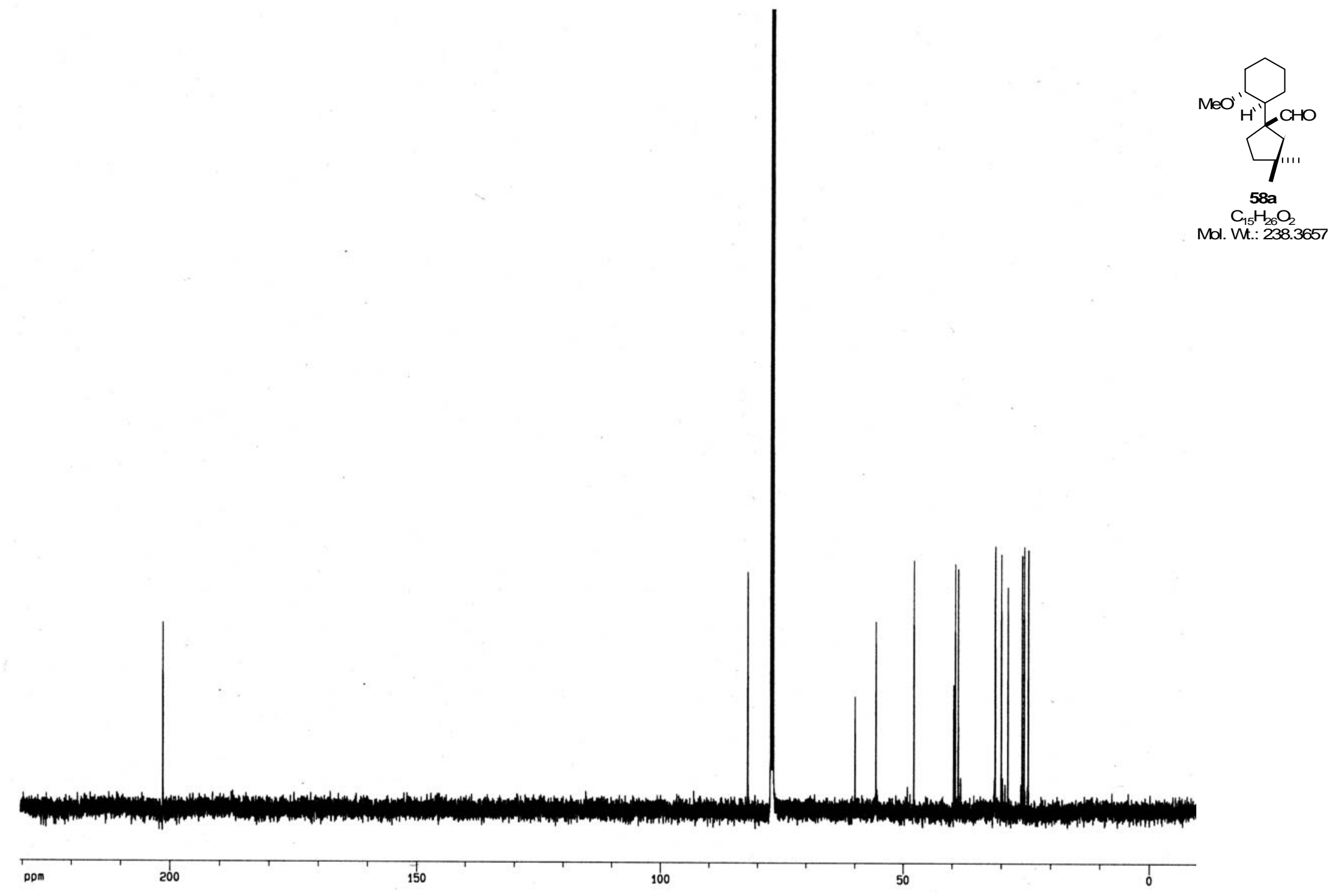


Overman and Velthuisen

S

102

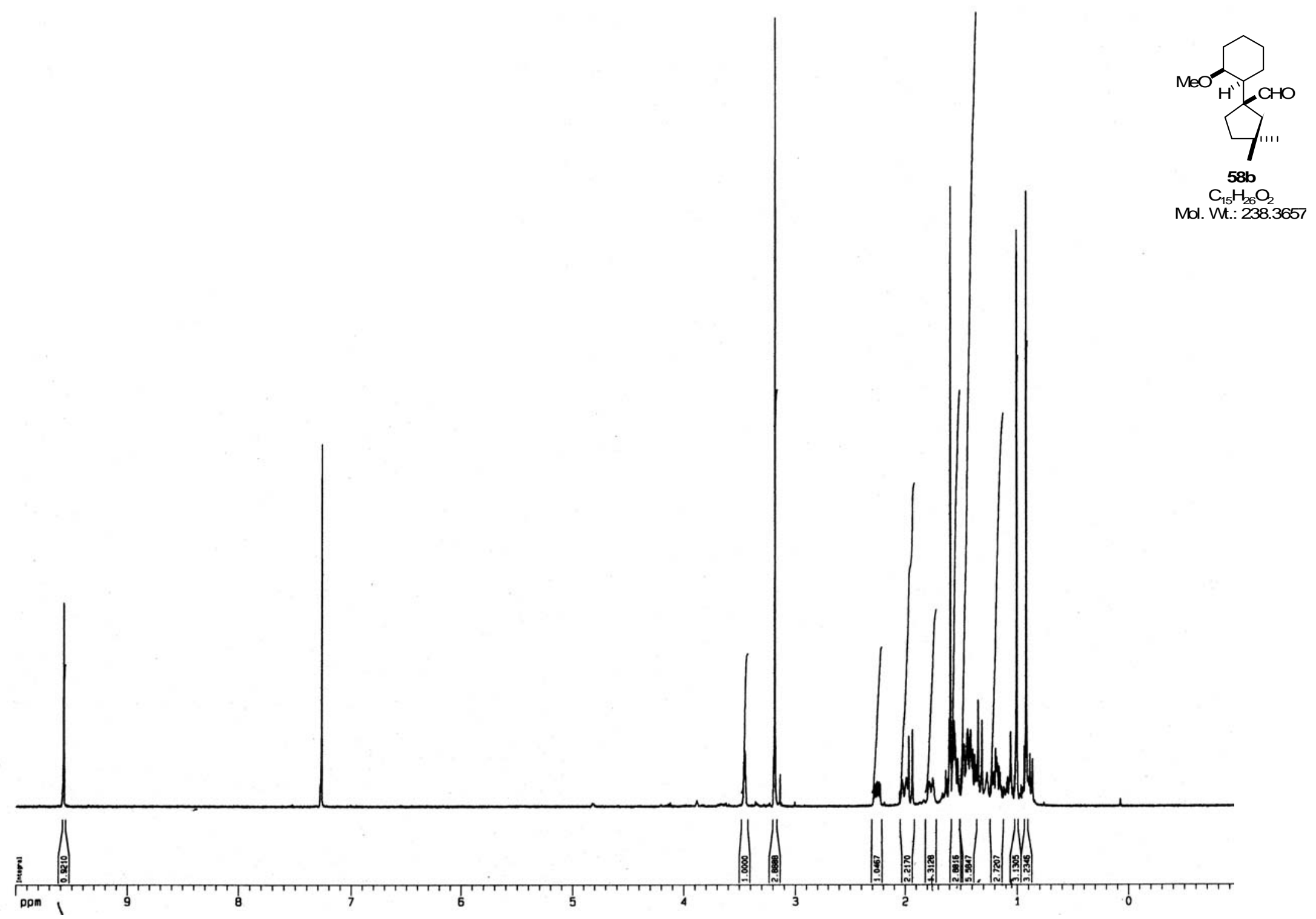




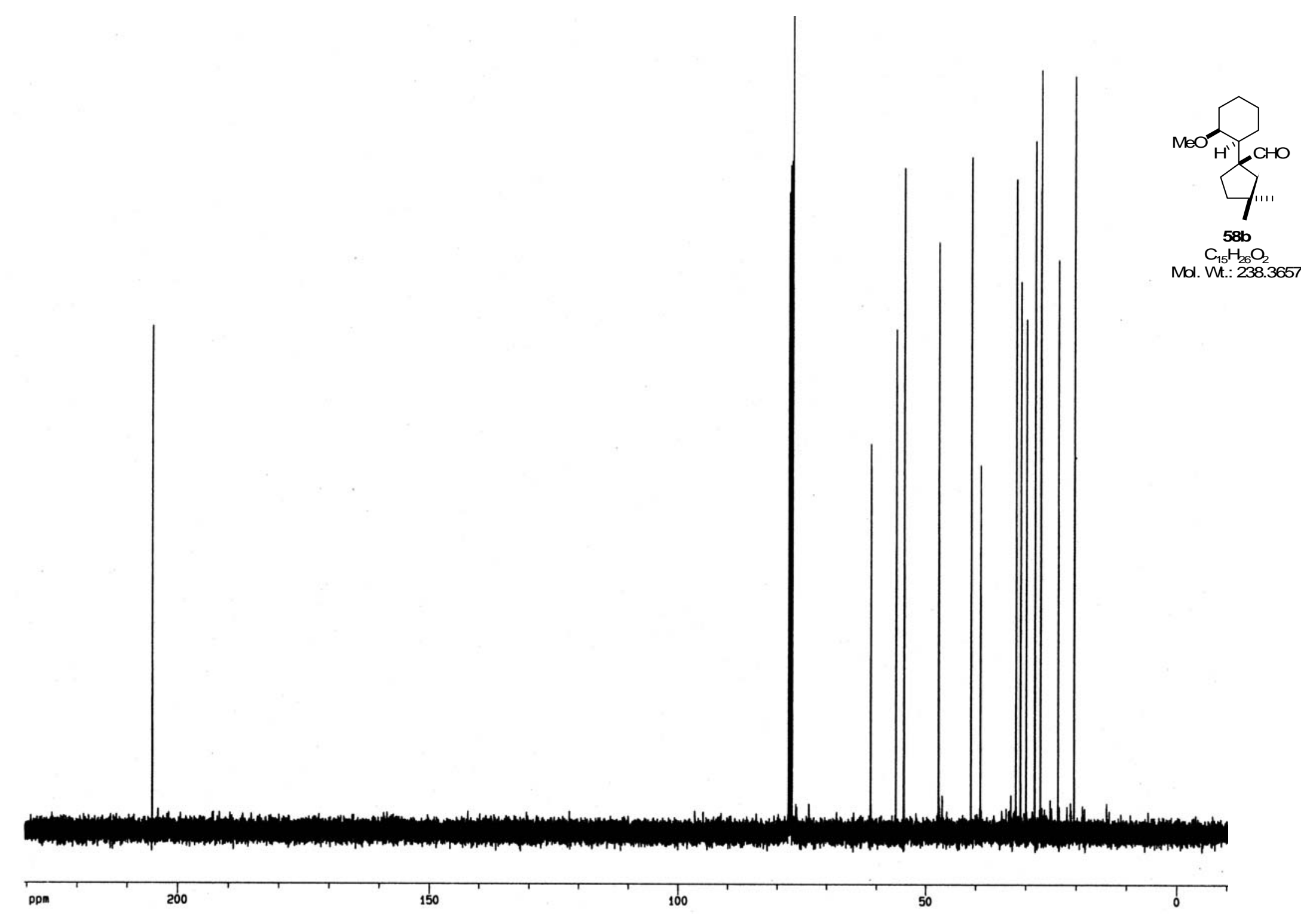




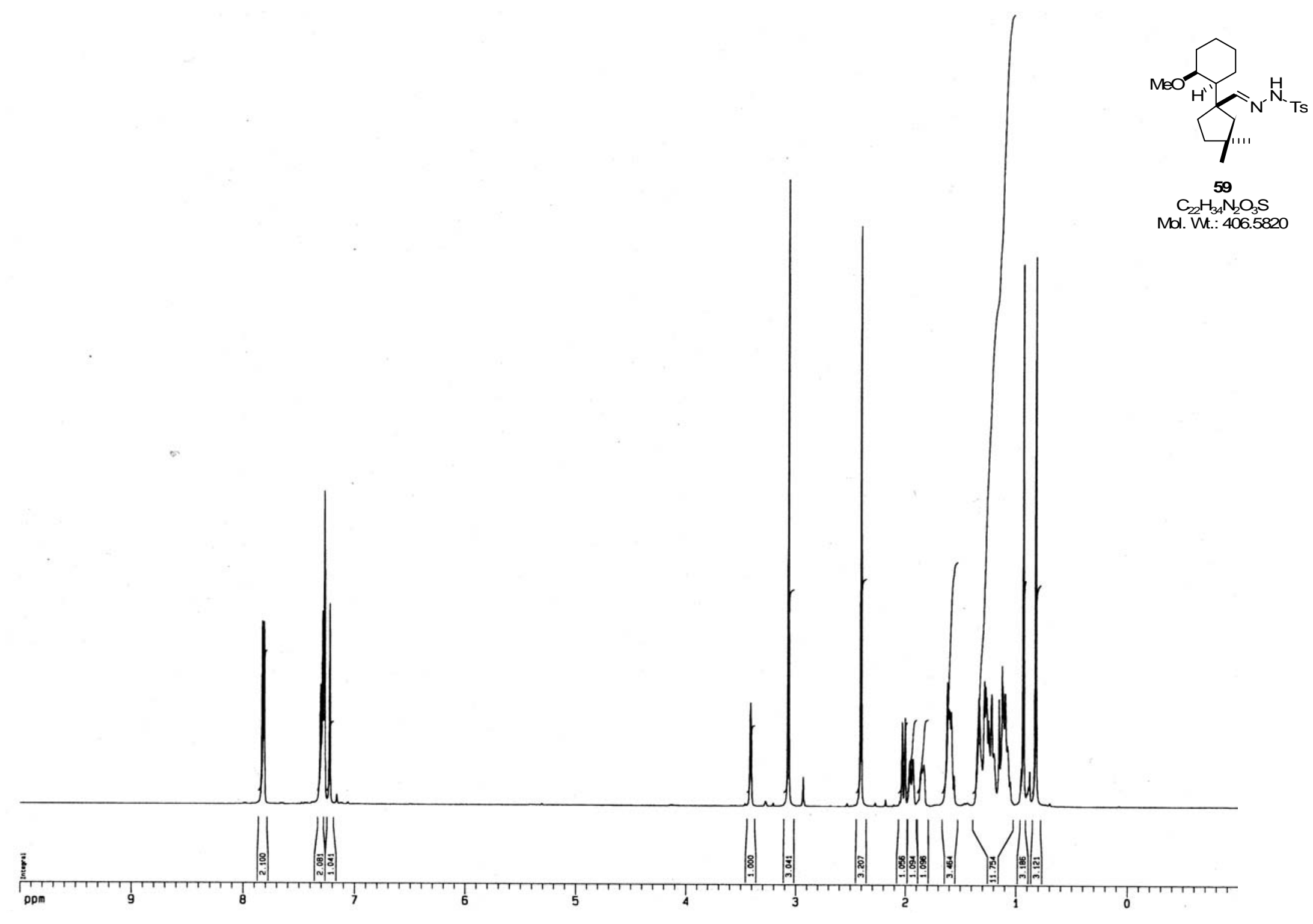




$$
\text { Uliti }
$$




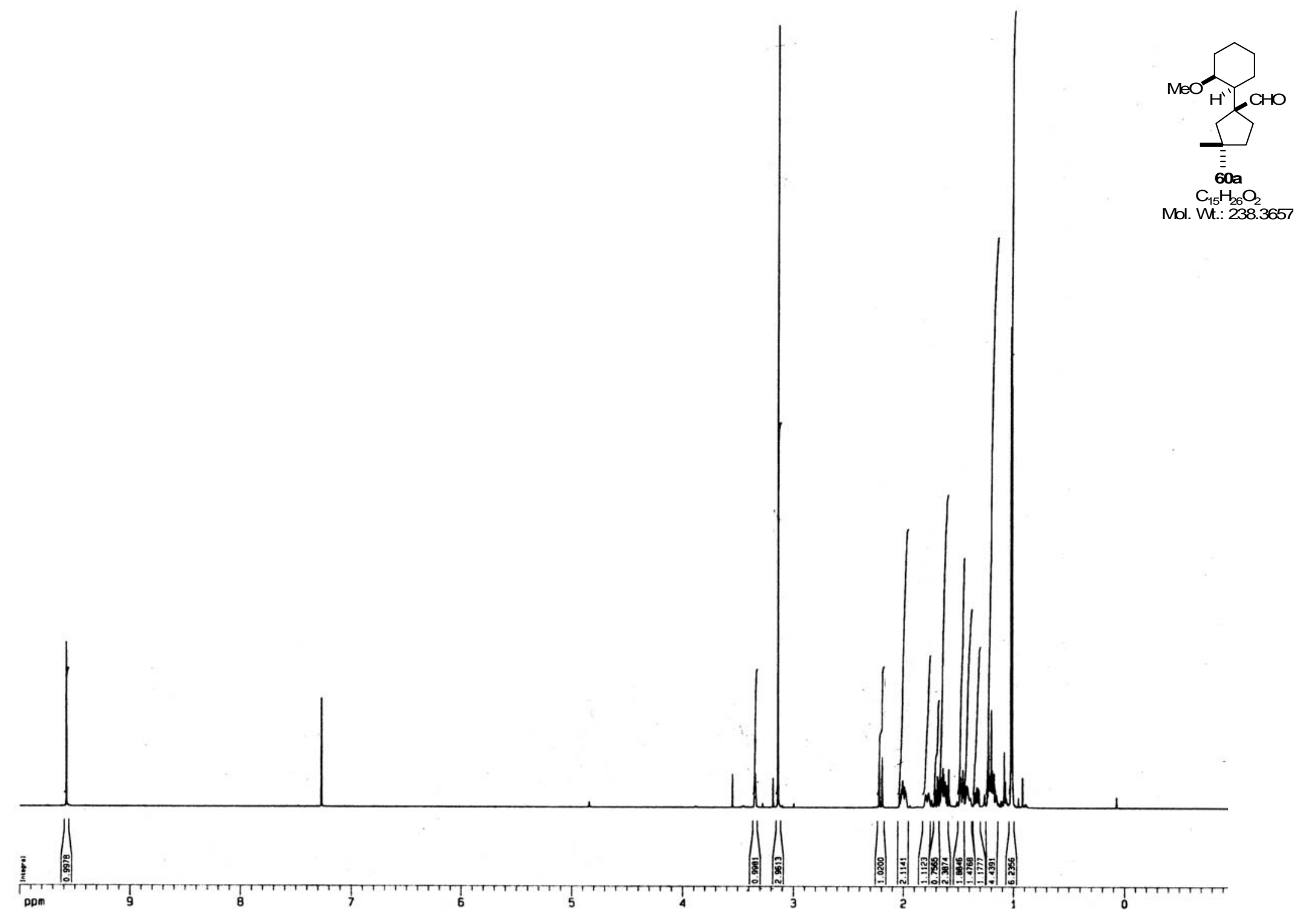




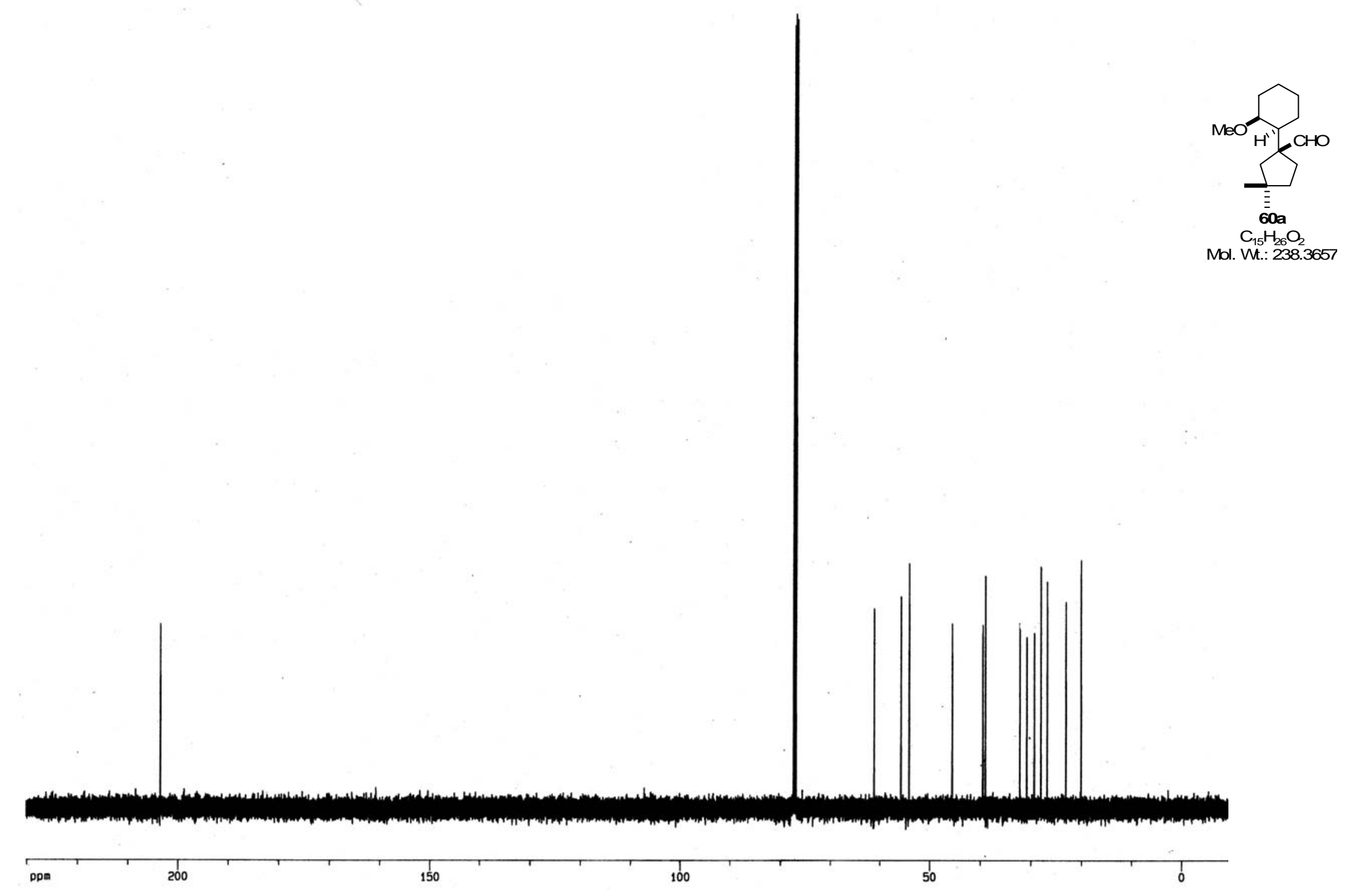




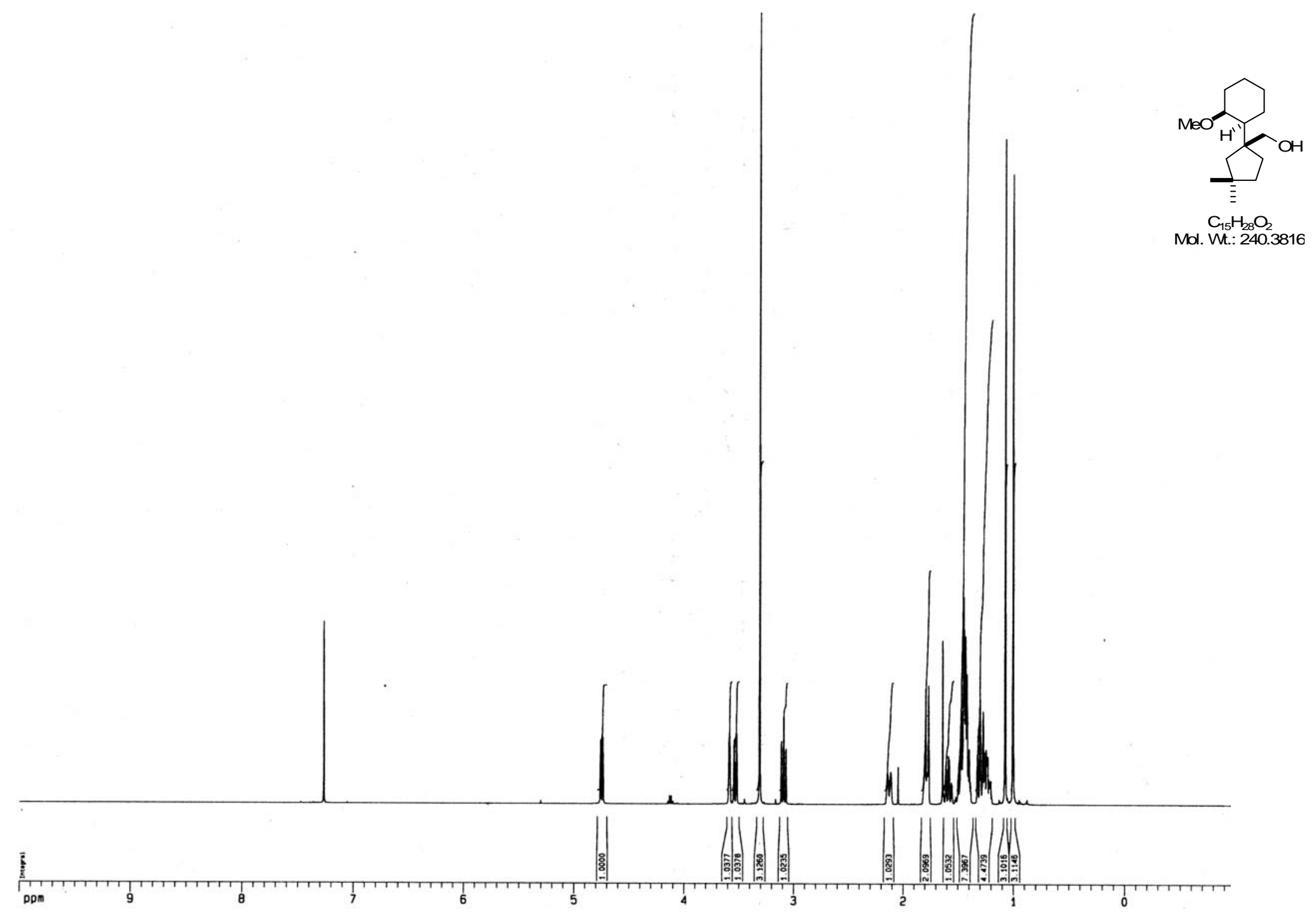




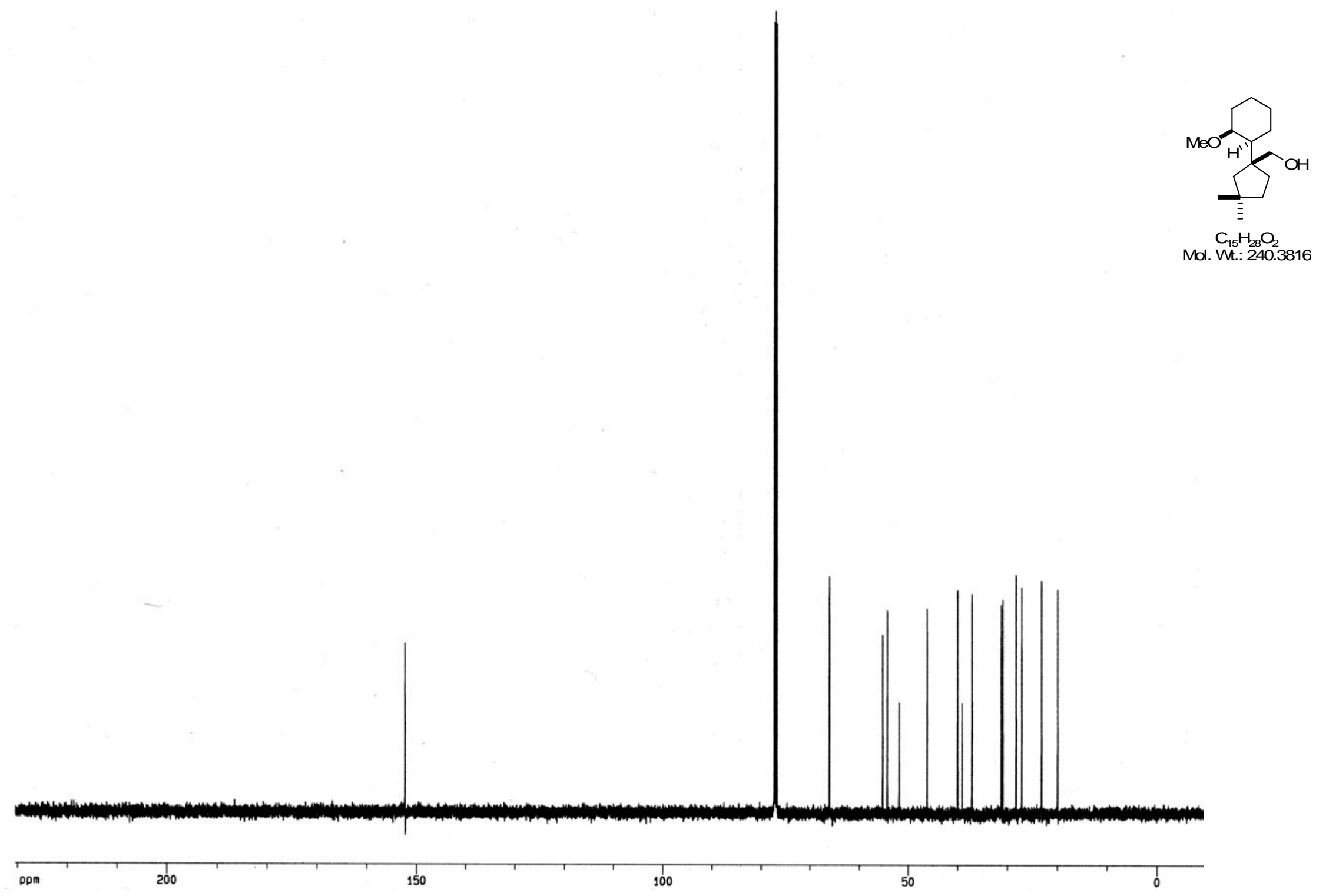




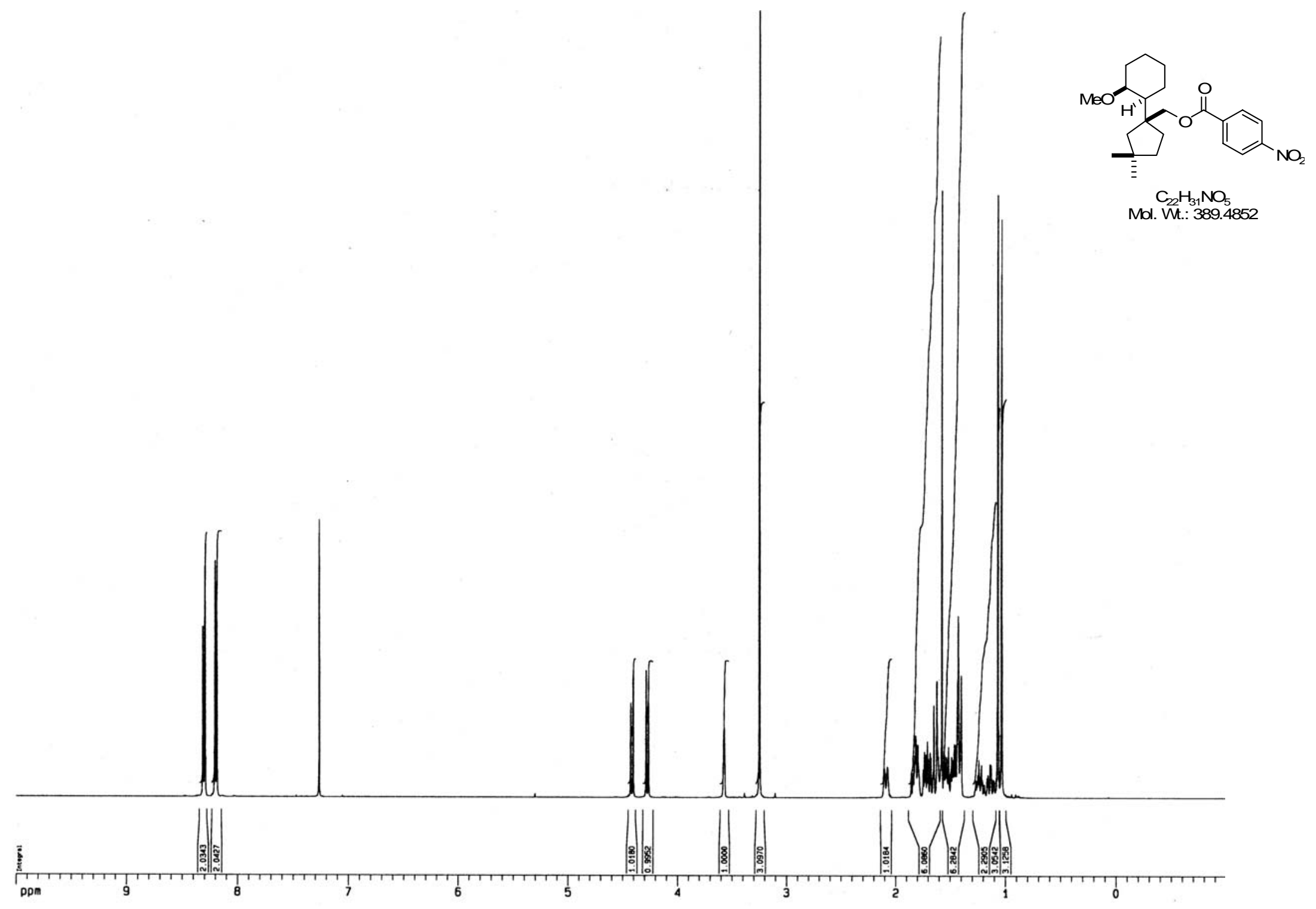




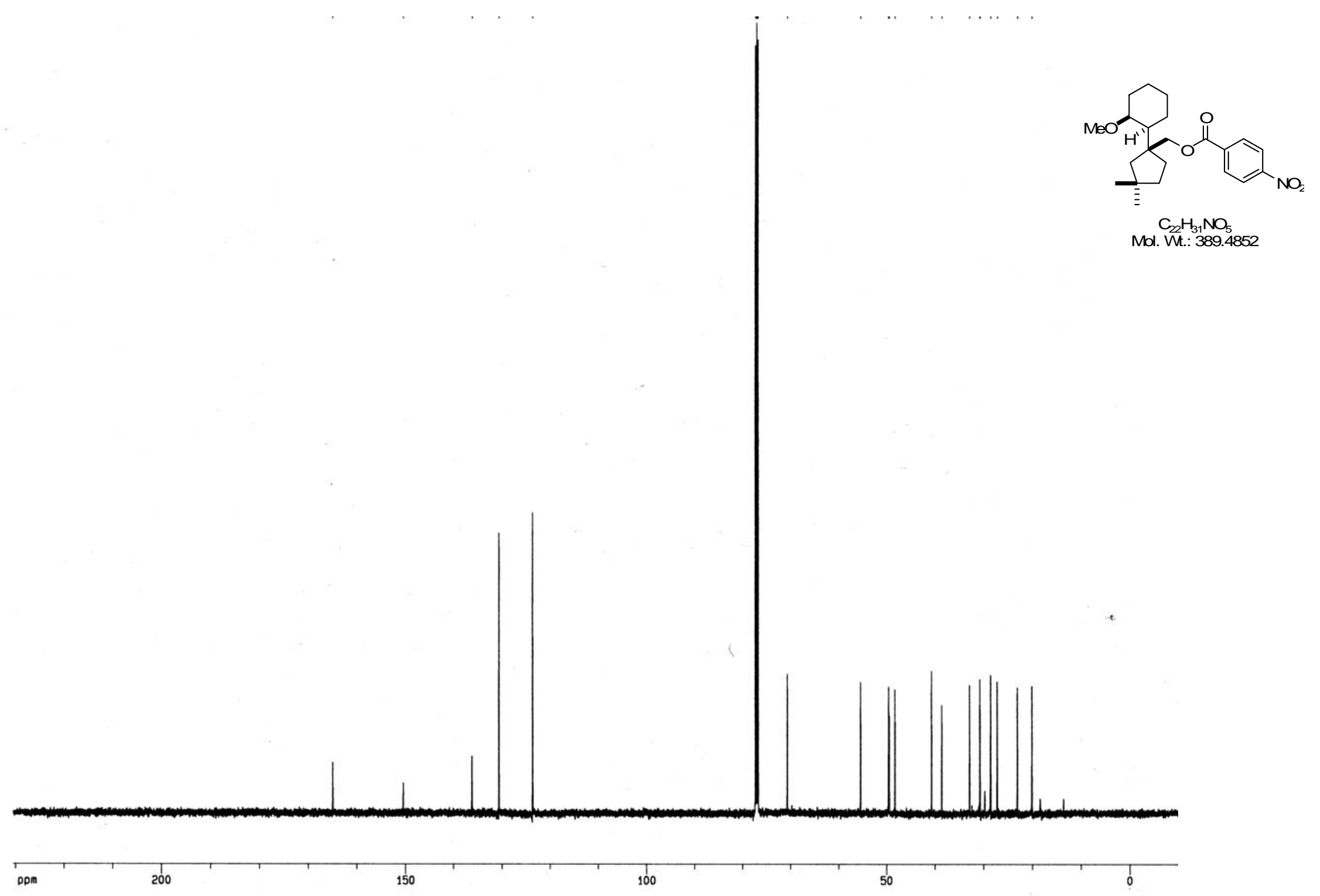


Data File $C:$ : HPCHEM 1 DARAAIEV-2958.D

$99.5 / 0.5$ hexanes/IPA isocratic

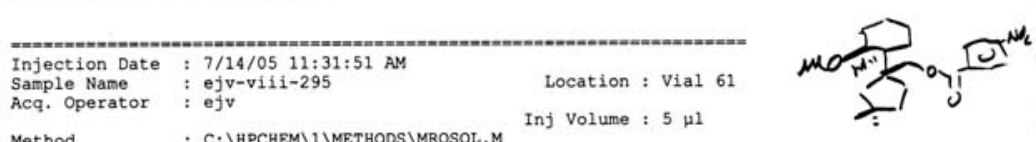

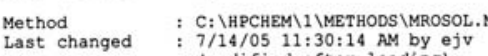
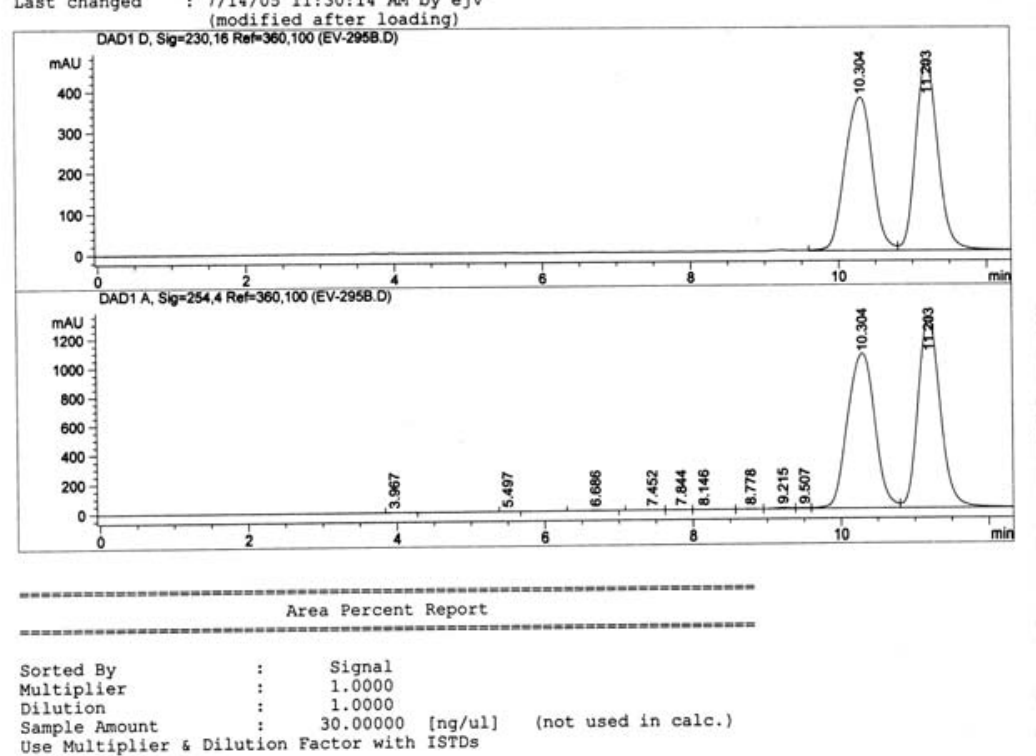

Sample anount
Use Multipliter

Signal 1: DAD1 D, Sig=230,16 Ref $-360,100$

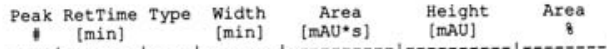

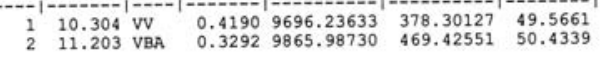

Totals :

$1.95622 \mathrm{e} \quad 847.72678$

Results obtained with enhanced integrator!
Sample Name: ejv-viii-295
Data File C: \HPCHEM 1 IDATA $99.5 / 0.5$ hexanes/IPA isocratic

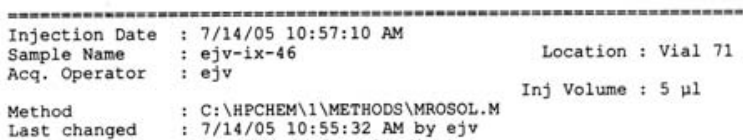

Sample Name: ejv-ix-46

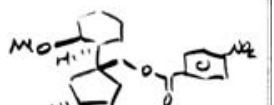

Method : C: \HPCHEMIIIMETHODS MMROSOL.M
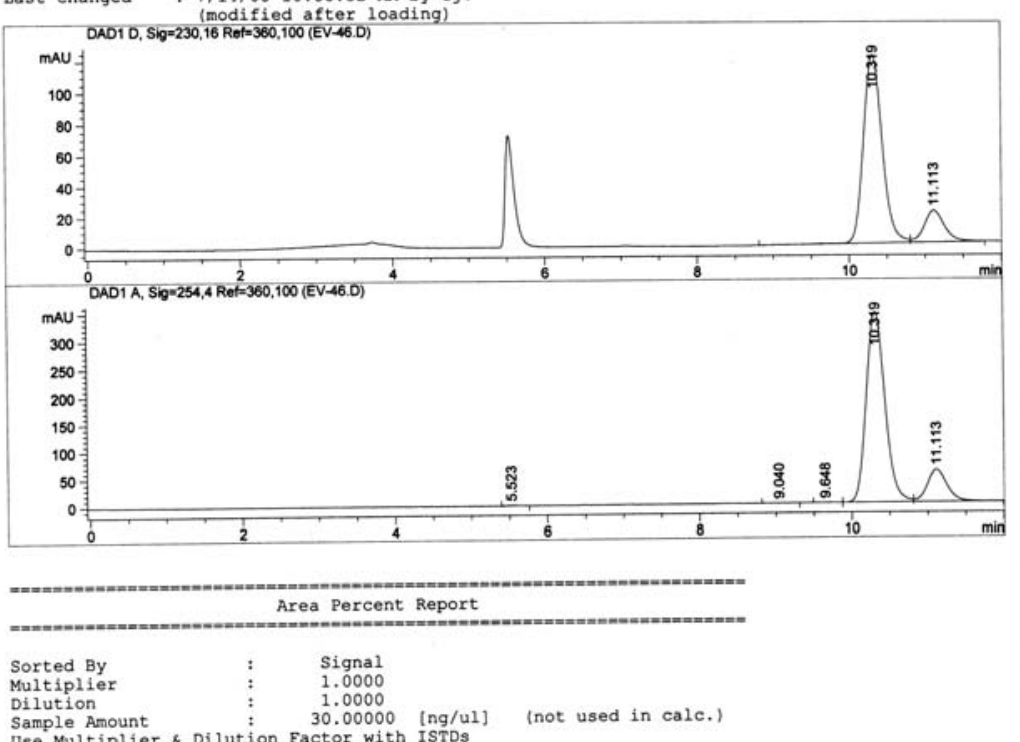

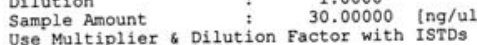

Signal 1: DAD1 D, Sig=230,16 Ref=360, 100

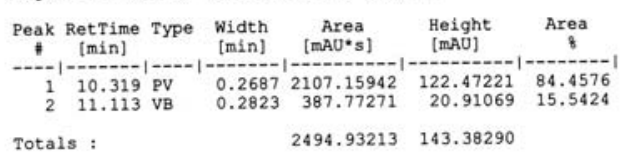

Results obtained with enhanced integrator! 


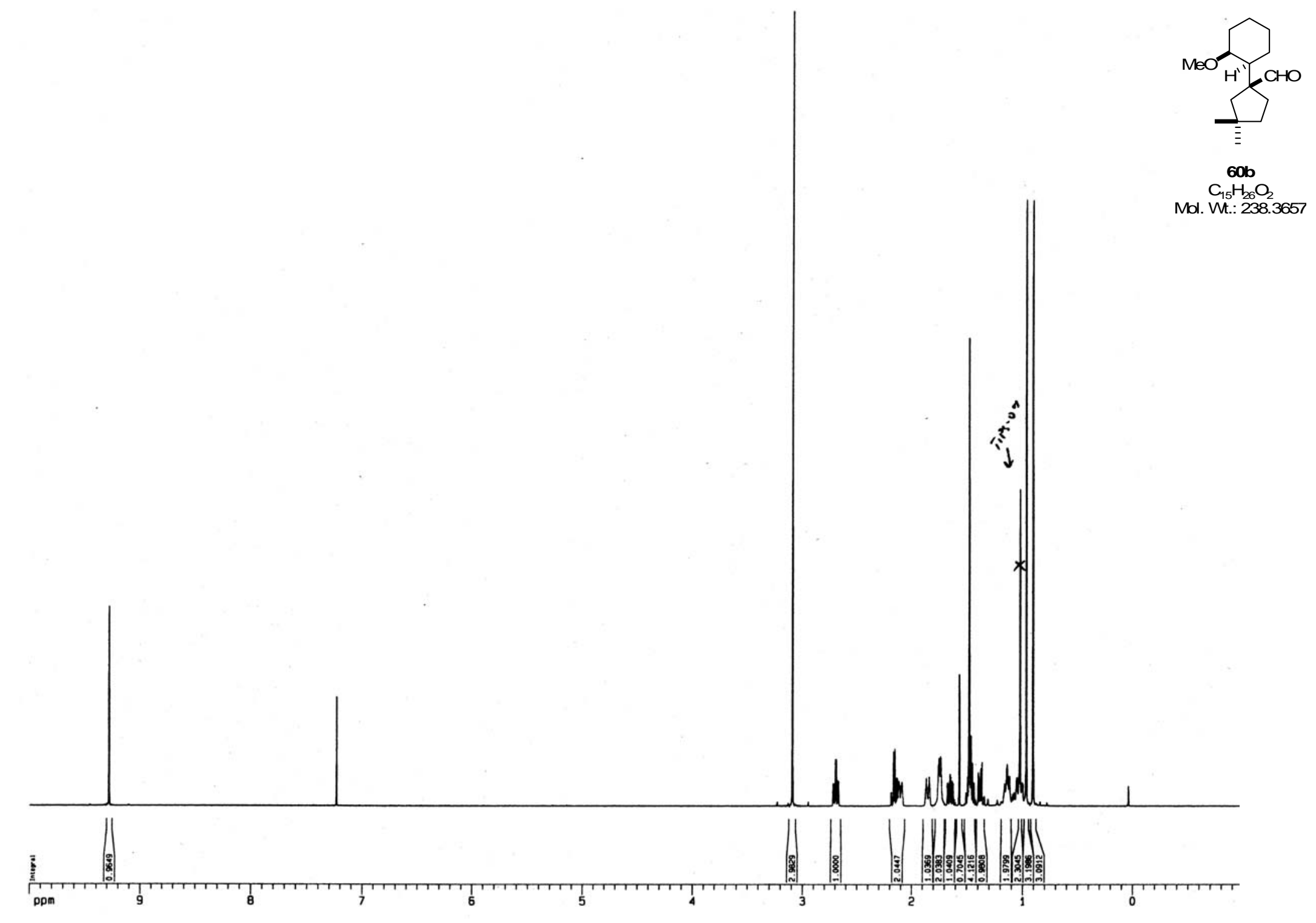




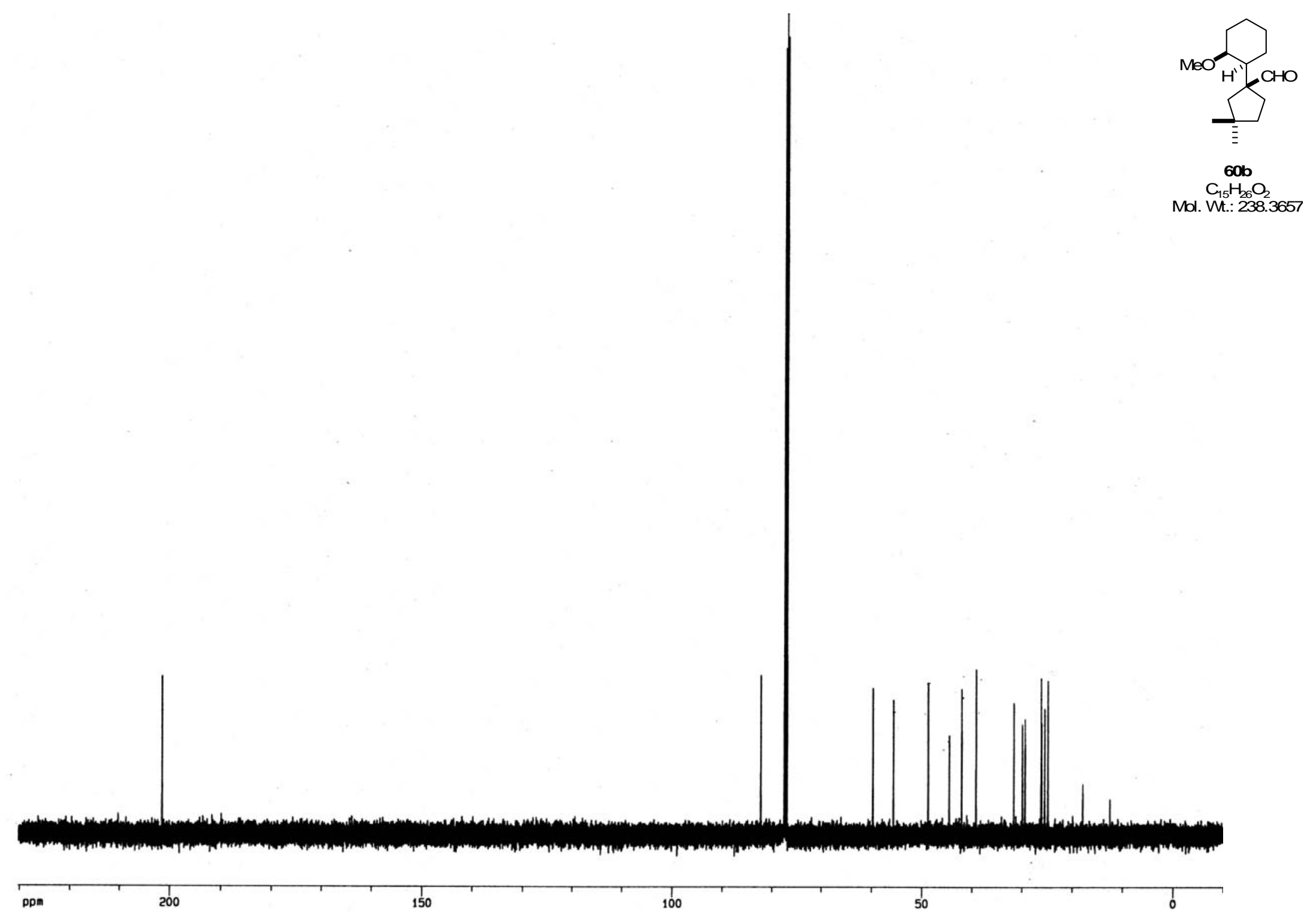




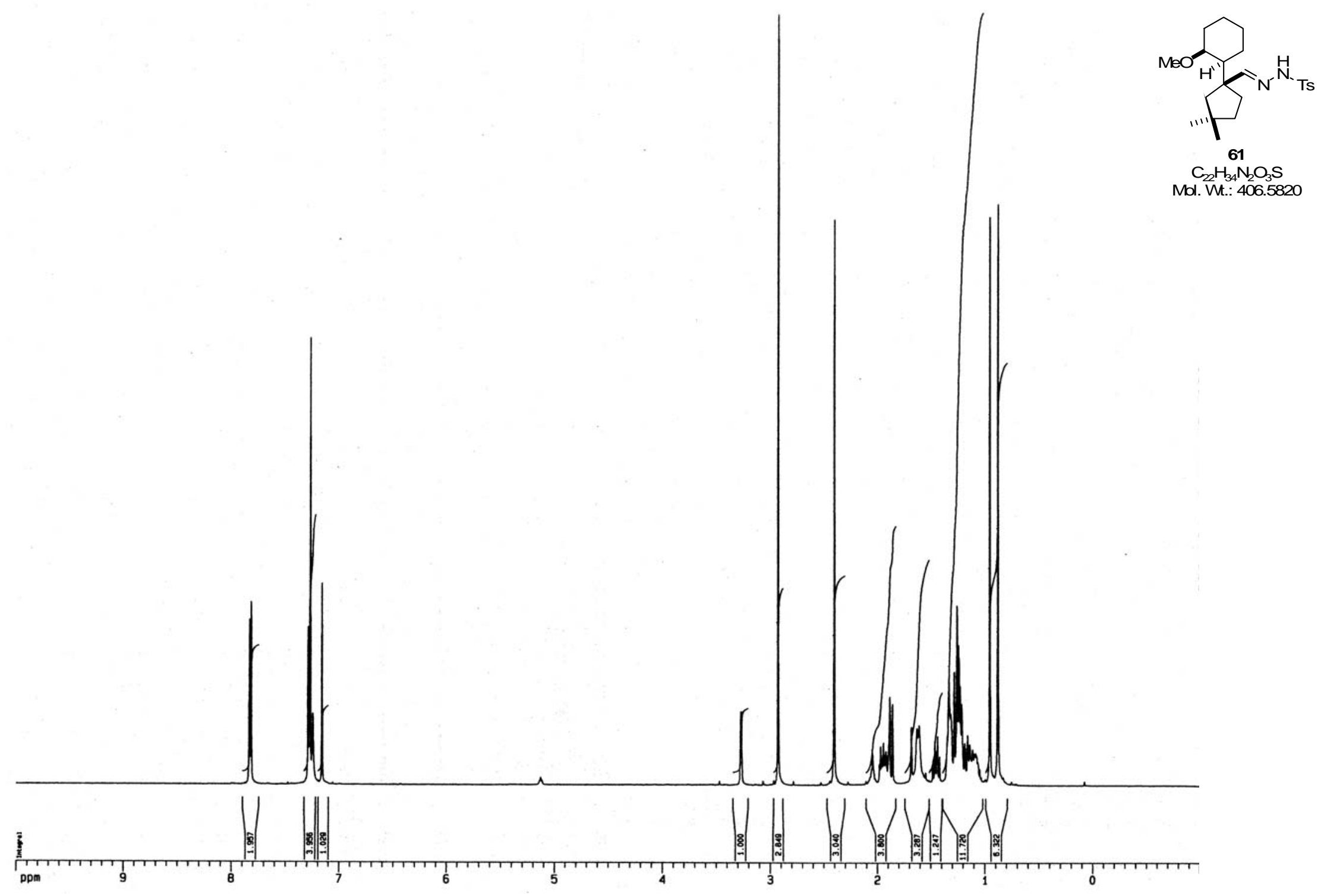




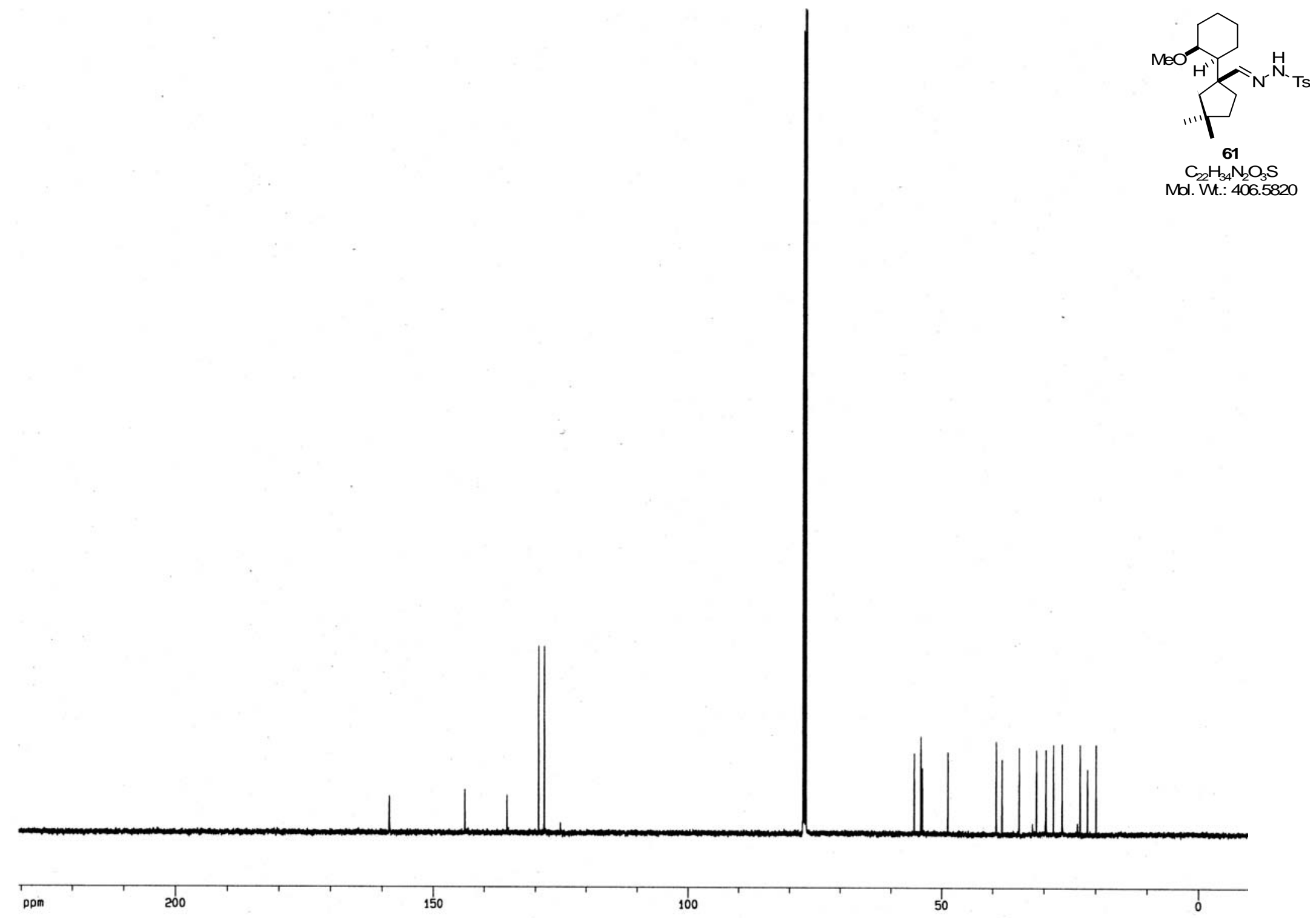


Overman and Velthuisen

S

117

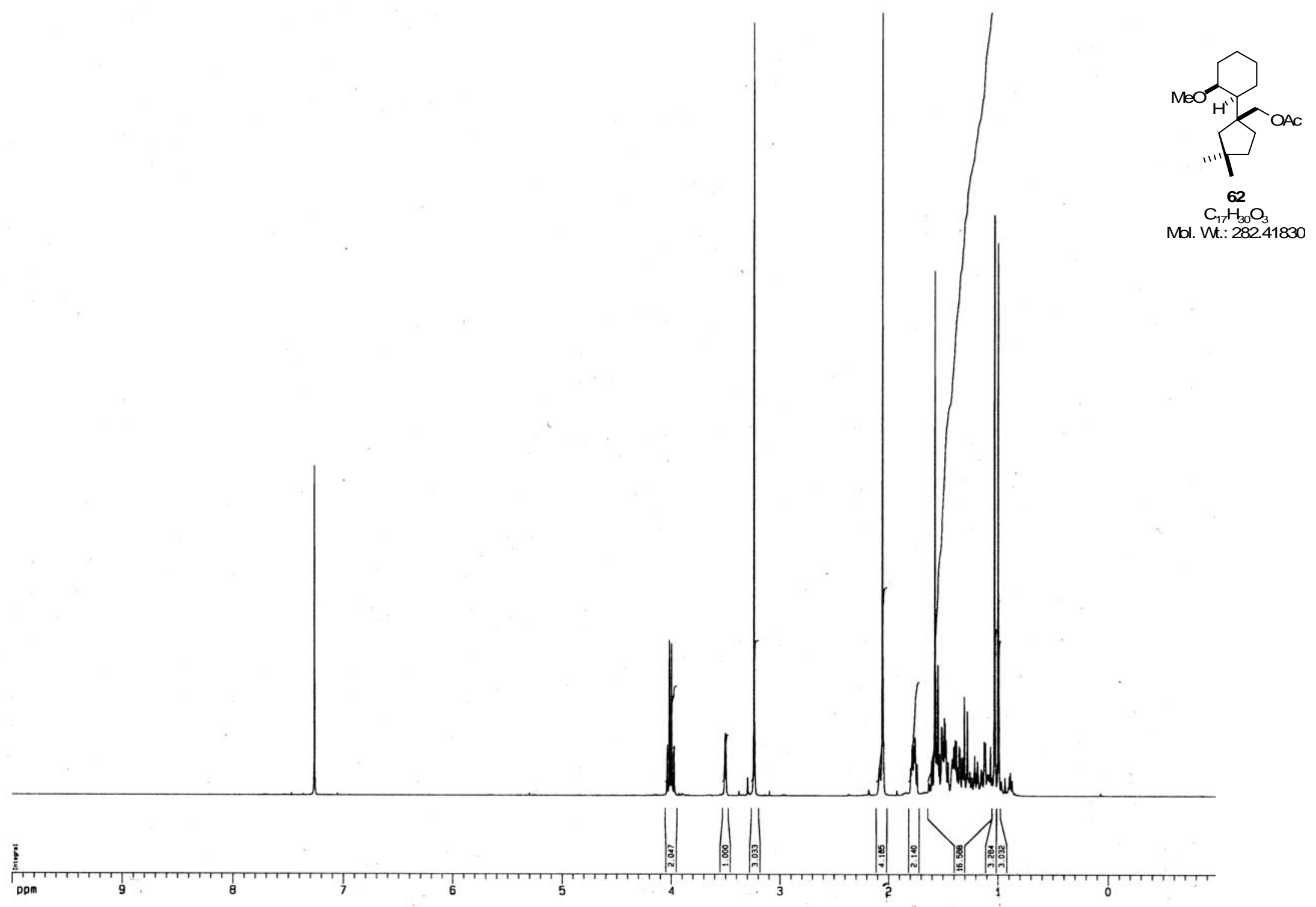




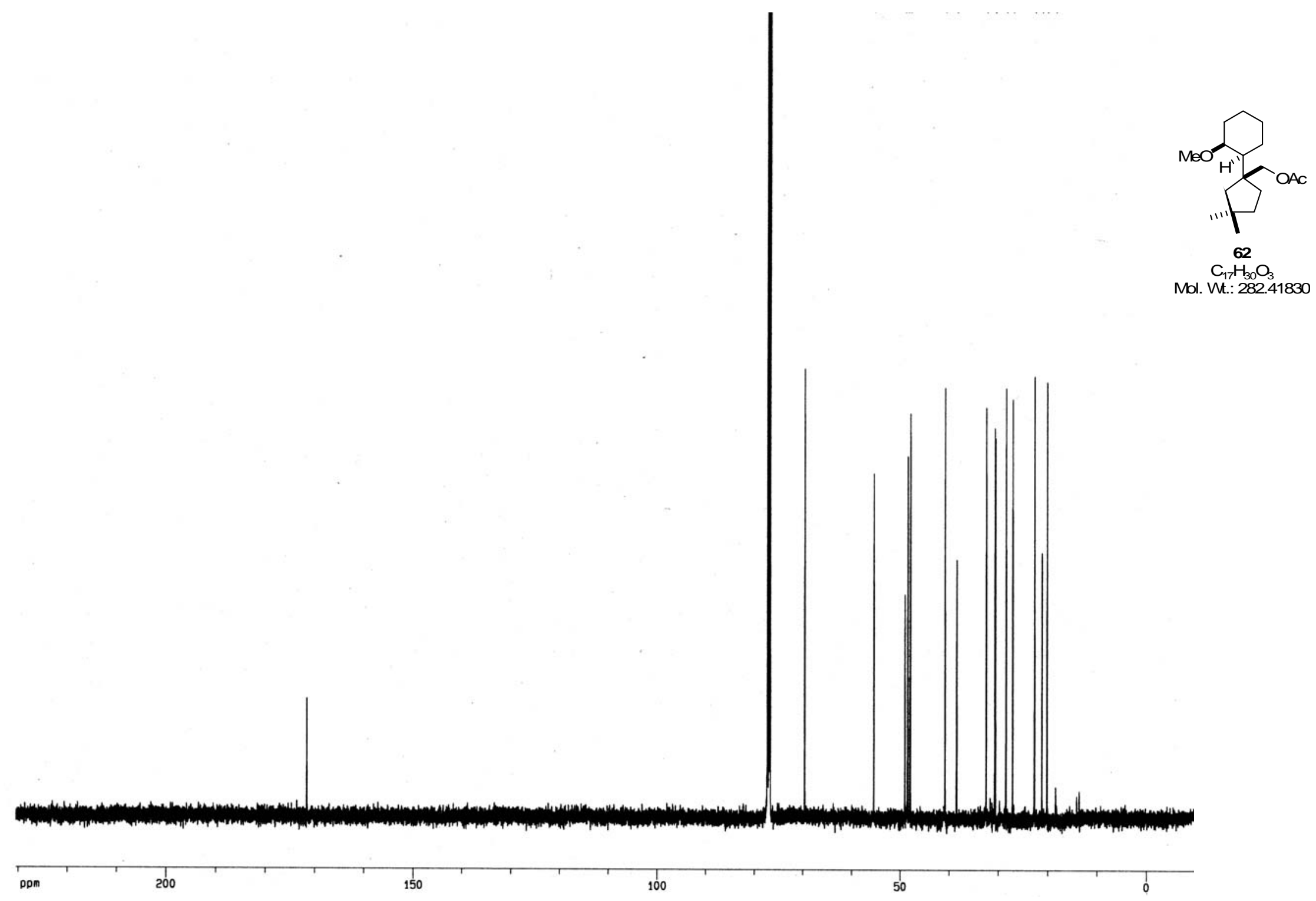




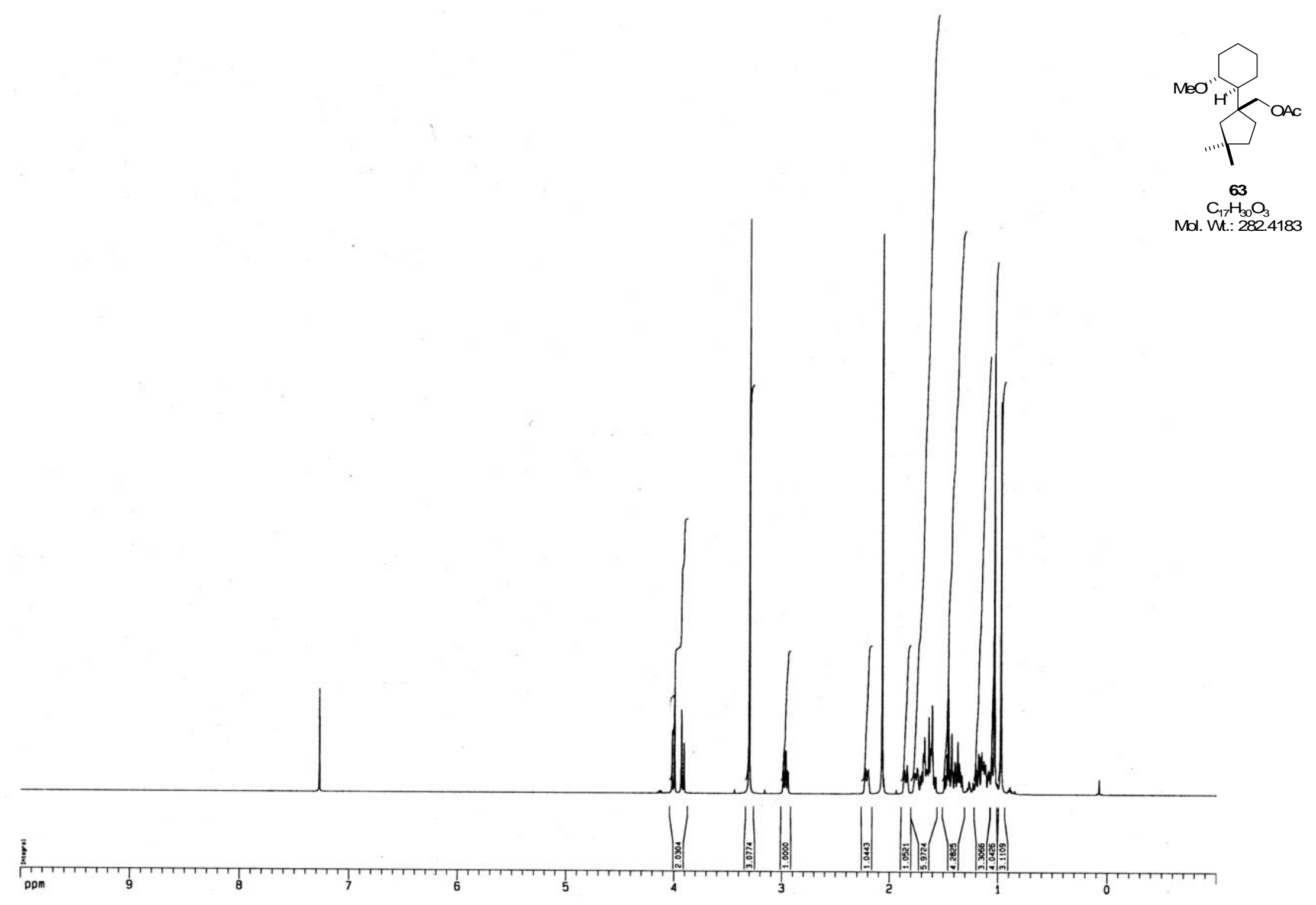




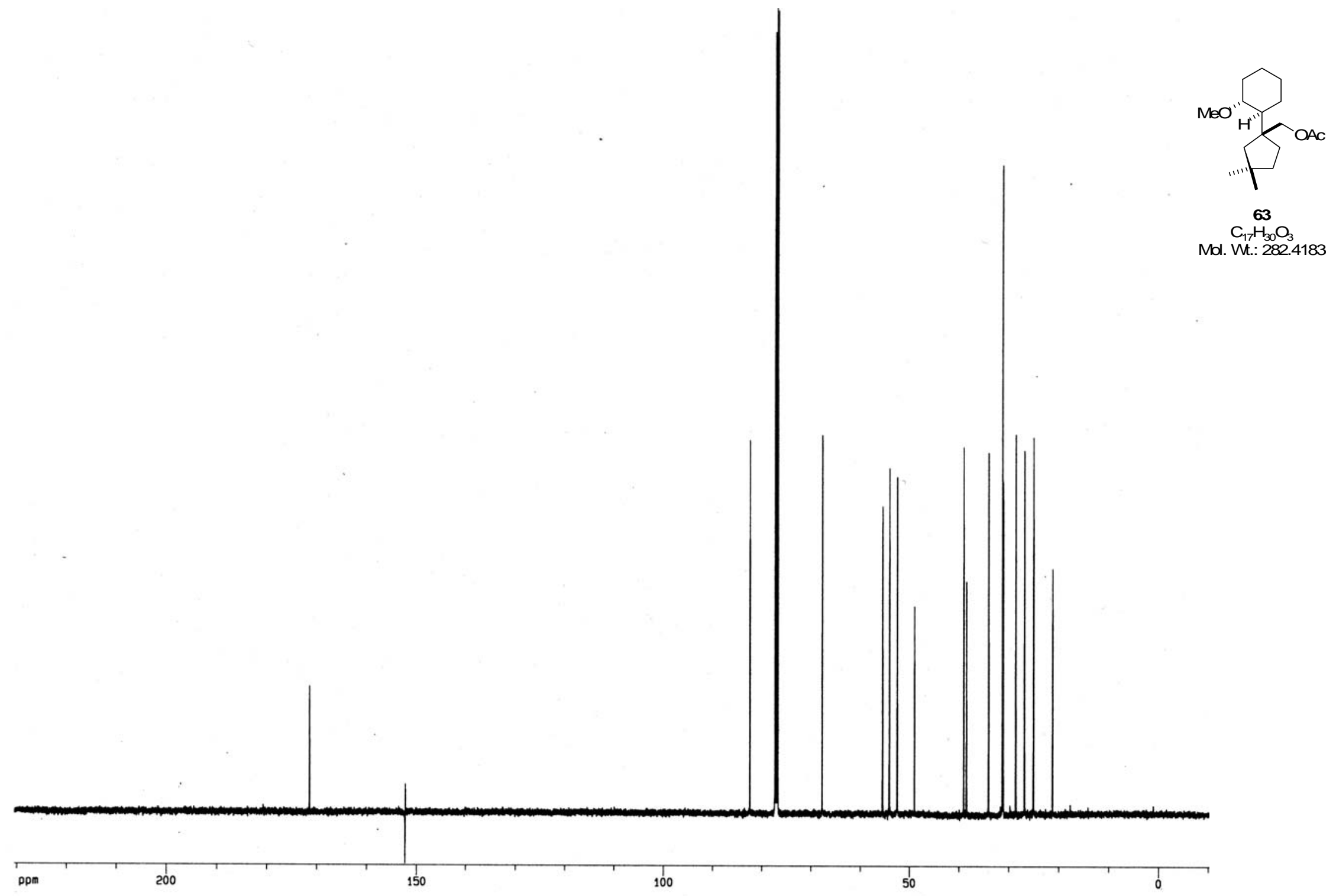




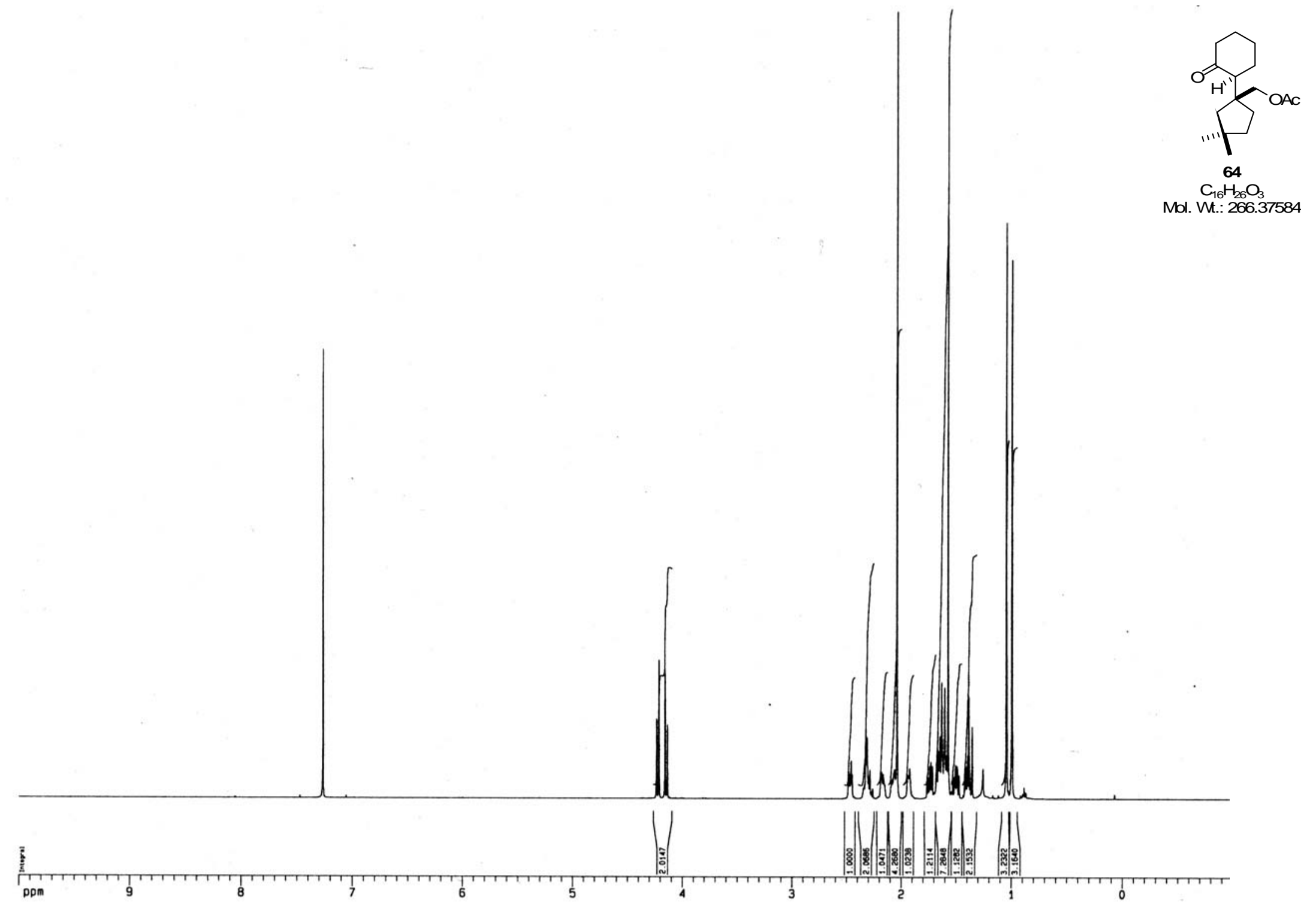




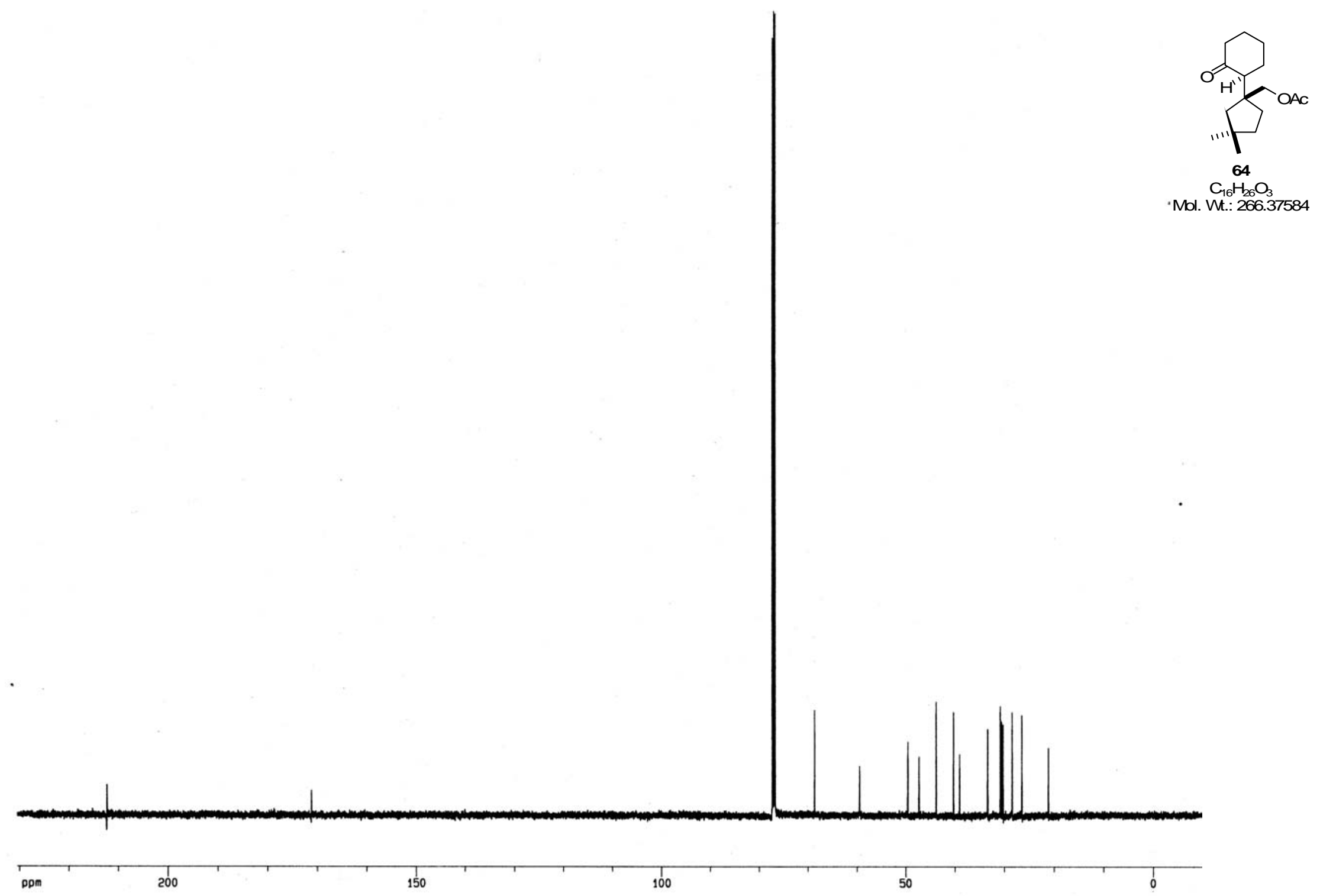

